\title{
In-depth study of polyamides containing galactaric acid-derived building blocks
}

Citation for published version (APA):

Wróblewska, A. A. (2018). In-depth study of polyamides containing galactaric acid-derived building blocks: from sugar beet to plastic. [Doctoral Thesis, Maastricht University]. ProefschriftMaken Maastricht. https://doi.org/10.26481/dis.20181127aw

Document status and date:

Published: 01/01/2018

DOI:

10.26481/dis.20181127aw

Document Version:

Publisher's PDF, also known as Version of record

\section{Please check the document version of this publication:}

- A submitted manuscript is the version of the article upon submission and before peer-review. There can be important differences between the submitted version and the official published version of record.

People interested in the research are advised to contact the author for the final version of the publication, or visit the DOI to the publisher's website.

- The final author version and the galley proof are versions of the publication after peer review.

- The final published version features the final layout of the paper including the volume, issue and page numbers.

Link to publication

\footnotetext{
General rights rights.

- You may freely distribute the URL identifying the publication in the public portal. please follow below link for the End User Agreement:

www.umlib.nl/taverne-license

Take down policy

If you believe that this document breaches copyright please contact us at:

repository@maastrichtuniversity.nl

providing details and we will investigate your claim.
}

Copyright and moral rights for the publications made accessible in the public portal are retained by the authors and/or other copyright owners and it is a condition of accessing publications that users recognise and abide by the legal requirements associated with these

- Users may download and print one copy of any publication from the public portal for the purpose of private study or research.

- You may not further distribute the material or use it for any profit-making activity or commercial gain

If the publication is distributed under the terms of Article $25 \mathrm{fa}$ of the Dutch Copyright Act, indicated by the "Taverne" license above, 
In-depth study of polyamides containing galactaric acid-derived building blocks

From sugar beet to plastic

Aleksandra Alicja Wróblewska 
In-depth study of polyamides containing galactaric acid-derived building blocks : From sugar beet to plastic

Wróblewska, A.A.

Maastricht University, 2018.

NUR 913

(C) 2018, Aleksandra Alicja Wróblewska

ISBN number: 978-94-6380-038-9

Printed by: ProefschriftMaken || www.proefschriftmaken.nl

Cover design by Dominka Gniazdek-Kaźmierczak 


\section{In-depth study of polyamides containing galactaric acid-derived building blocks}

From sugar beet to plastic

\section{DISSERTATION}

to obtain the degree of Doctor at the Maastricht University, on the authority of the Rector Magnificus,

Prof.dr. Rianne M. Letschert

in accordance with the decision of the Board of Deans,

to be defended in public

on Tuesday $27^{\text {th }}$ November 2018, at 14:00 hours

by

Aleksandra Alicja Wróblewska 
Supervisor:

Prof. Dr. Ir. Stefaan De Wildeman

Co-supervisor:

Dr. Katrien Bernaerts

Assessment Committee:

Prof. Dr. Lorenzo Moroni (chair)

Dr. Hanne Diliën

Prof. Dr. Francesco Picchioni, Rijksuniversiteit Groningen

Dr. Sepas Satayesh, Philips (Drachten, NL)

This work was supported by the "Samenwerkingsverband Noord-Nederland" (SNN; project T3006 / Beets to Biopolymers). 


\begin{tabular}{|c|c|}
\hline$A C N$ & Acetonitrile \\
\hline ATR-IR & Attenuated total reflection-infrared \\
\hline$B n A$ & Benzylamine \\
\hline $\boldsymbol{\oplus}$ & Dispersity \\
\hline $\boldsymbol{D A}$ & Dodecanedioic acid \\
\hline DAPIS & Isosorbide(bis(propan-1-amine)) \\
\hline$D D D A$ & 1,12-dodecanediamine \\
\hline$D E A$ & Diethyl adipate \\
\hline$D E P T$ & Distortionless enhancement by polarization transfer (NMR technique) \\
\hline$D F T$ & Density functional theory \\
\hline$D M A$ & Dimethyl adipate \\
\hline DMSO & Dimethyl sulfoxide \\
\hline$D P$ & Degree of polymerization \\
\hline$D S C$ & Differential scanning calorimetry \\
\hline E modulus & Young's modulus \\
\hline$f_{a v}$ & Average functionality \\
\hline$F D C A$ & Furandicarboxylic acid \\
\hline$F T-I R$ & Fourier-transform infrared spectroscopy \\
\hline $\boldsymbol{G}$ & Gibbs free energy \\
\hline$G$ & Storage modulus \\
\hline$G^{\prime}$, & Loss modulus \\
\hline Gal & Galactaric acid motif \\
\hline Gal-OEt & Diethyl ester of galactaric acid \\
\hline Gal-OMe & Dimethylester of galactaric acid \\
\hline GalX & 2,3:4,5-di- $O$-acetalized galactaric acid derivative \\
\hline GalXBn & 2,3:4,5-di- $O$-benzylidene-galactaric acid derivative \\
\hline GalXBnF & 2,3:4,5-di-O-3-fluorobenzylidene-galactaric acid derivative \\
\hline GalXH & 2,3:4,5-di- $O$-methylene-galactaric acid derivative \\
\hline GalXHex & 2,3:4,5-di- $O$-hexylidene-galactaric acid derivative \\
\hline GalXMe & 2,3:4,5-di- $O$-isopropylidene-galactaric acid derivative \\
\hline GalX-NHX & 2,3:4,5-di-O-acetalized N-modified galactaramid \\
\hline GalX-ol & 2,3:4,5-di- $O$-acetalized galactitol \\
\hline GluX & Biacetalized D-glucose derivatives \\
\hline$G P C$ & Gel permeation chromatography \\
\hline $\boldsymbol{H}$ & Enthalpy \\
\hline HFIP & 1,1,1,3,3,3-hexafluoro-2-propanol \\
\hline HMBC & Heteronuclear multiple bond correlation (NMR technique) \\
\hline$H M D A$ & 1,6-hexamethylenediamine \\
\hline$H S Q C$ & Heteronuclear single bond connectivity (NMR technique) \\
\hline$I D C A$ & Isoidide dicarboxylic acid \\
\hline
\end{tabular}




\begin{tabular}{|c|c|}
\hline$I P D A$ & 5-Amino-1,3,3-trimethylcyclohexanemethanamine \\
\hline$L C$ & Liquid chromatography \\
\hline Maldi-ToF & Matrix assisted laser desorption/ionization - time of flight \\
\hline $\operatorname{Man} X$ & Biacetalized D-mannose derivatives \\
\hline$M_{n}$ & Number average molecular weight \\
\hline$M S$ & Mass spectroscopy \\
\hline$M X D$ & 1,3-phenylenedimethanamine \\
\hline NMP & N-methylpirrolidone \\
\hline NMR & Nuclear magnetic resonance \\
\hline$O X D A$ & 4,4'-oxydianiline \\
\hline p/conv & Conversion \\
\hline$P 1$ or $P 2$ & Products \\
\hline $\boldsymbol{P A}$ & polyamide \\
\hline$P A C M$ & 4,4'- methylenebis(cyclohexylamine) \\
\hline $\mathrm{PACM}-\mathrm{CH}_{3}$ & 4,4'- methylenebis(2-methylcyclohexylamine) \\
\hline$P E F$ & Poly(ethylene furanoate) \\
\hline PES & Potential energy surface \\
\hline$P E T$ & Poly(ethylene terephthalate) \\
\hline PFP & Pentafluorophenol \\
\hline$P L A$ & Poly(lactic acid) \\
\hline$P P D A$ & p-phenylenediamine \\
\hline$p-T S A$ & p-toluenesulfonic acid \\
\hline $\boldsymbol{R} \boldsymbol{U}$ & Repeating unit \\
\hline$S$ & Entropy \\
\hline$S 1$ & Substrates \\
\hline$S \boldsymbol{A}$ & Sebacic acid \\
\hline$S E M$ & Scanning electron microscopy \\
\hline $\boldsymbol{T}$ & Temperature \\
\hline$t d F T-I R$ & Temperature-dependent FT-IR \\
\hline$T_{g}$ & Glass transition temperature \\
\hline$T G A$ & Thermogravimetric analysis \\
\hline$T H F$ & Tetrahydrofurane \\
\hline$T_{m}$ & Melting temperature \\
\hline$T_{\max }$ & Temperature corresponding to maximum rate of weight lo \\
\hline$T S$ & Transition state \\
\hline$T_{x} \%$ & Temperature corresponding to $\mathrm{x} \%$ weight loss \\
\hline$W A X D$ & Wide angle $\mathrm{X}$-ray diffraction \\
\hline$\gamma_{(F T-I R)}$ & Skeletal vibration \\
\hline$\delta(N M R$ spectra $)$ & Chemical shift \\
\hline$\delta_{(F T-I R)}$ & Deformation \\
\hline
\end{tabular}




\begin{tabular}{l|l}
$\boldsymbol{\Delta} \boldsymbol{H}_{\boldsymbol{m}}$ & Enthalpy of melting \\
$\boldsymbol{\eta}^{*}$ & Complex viscosity \\
$\boldsymbol{v}_{(\boldsymbol{F T}-\boldsymbol{I R})}$ & Stretching vibration \\
$\boldsymbol{X}$ & Degree of crystallinity
\end{tabular}





\section{TABLE OF CONTENTS}

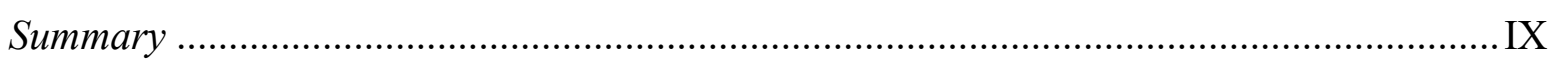

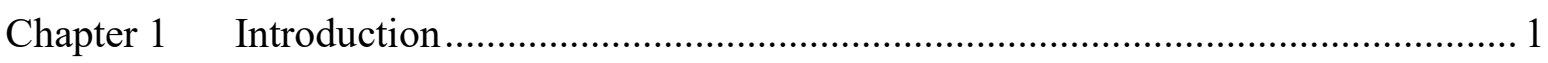

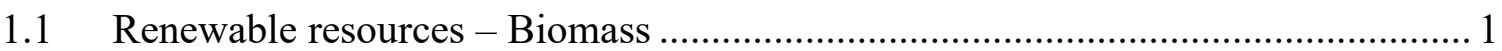

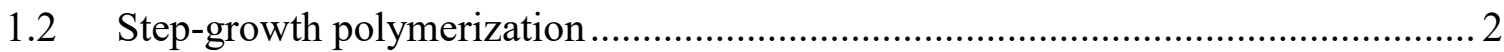

1.3 Sugar beet as a source of monomers utilized in polymer production ...................... 4

1.4 Biacetalized galactaric acid derivatives ……………………………………....

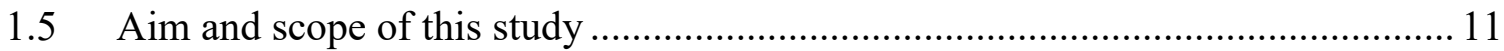

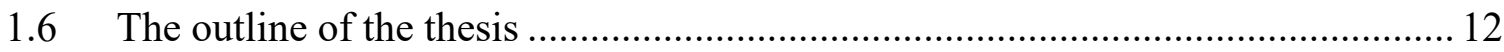

Chapter 2 Properties of GalX polyamides …………………………………………... 17

$2.1 \quad$ Introduction ................................................................................................ 19

2.2 The synthesis and properties of homopolyamides with different diamines ...........21

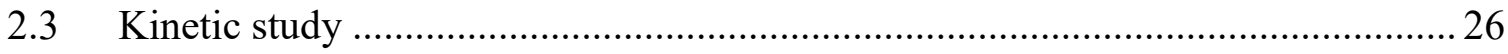

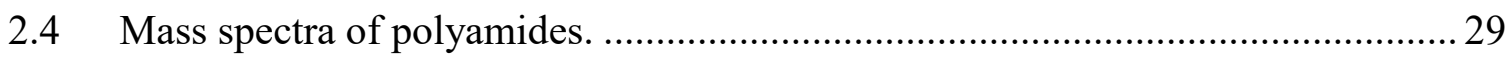

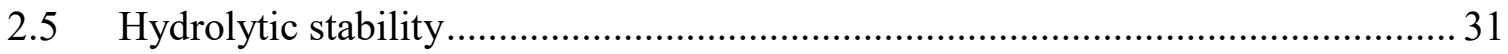

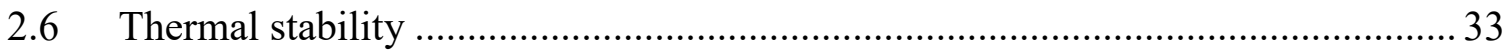

2.7 Rheological properties of GalX polymers.............................................................. 34

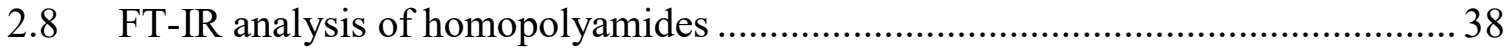

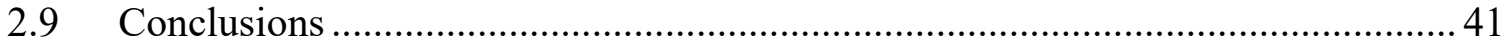

2.10 Materials and methods ................................................................................. 41

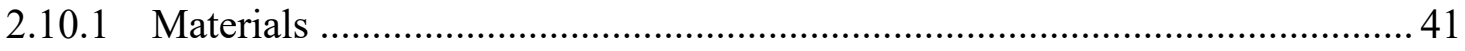

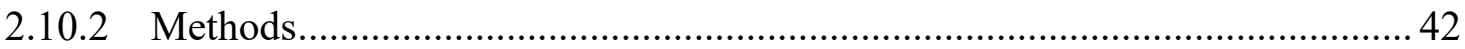

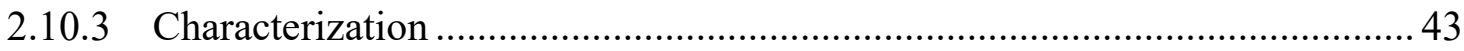

Chapter 3 Structure-property relations in GalX-based monomers and polymers............ 47

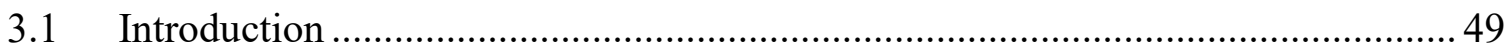

3.2 The synthesis of GalX derivatives and PAs therefrom ……………......................50 
3.3 Properties of polyamides made with Different GalX Derivatives

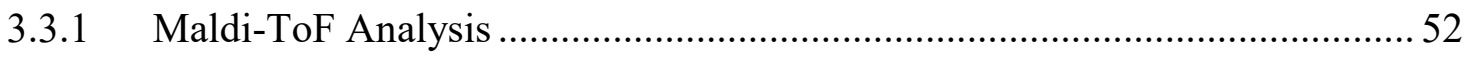

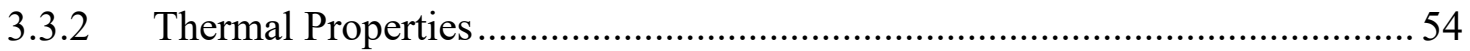

3.3.3 The Reactivity of the Carboxyl Group............................................................ 54

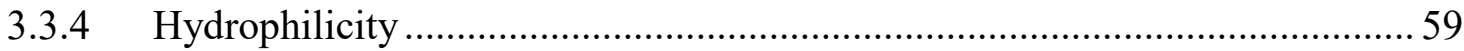

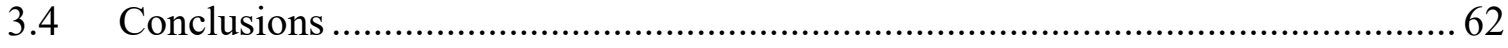

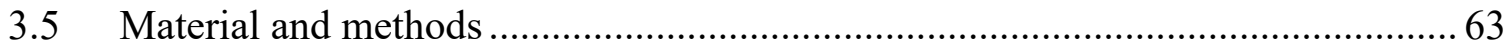

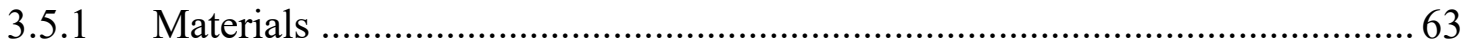

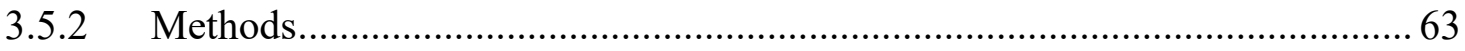

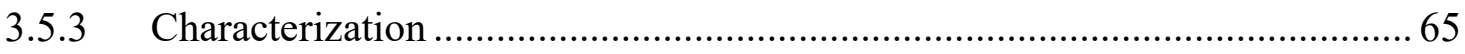

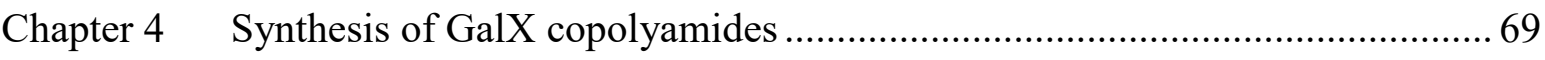

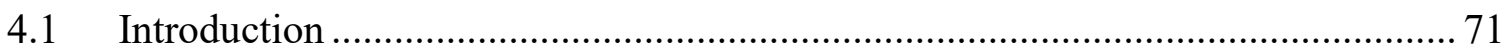

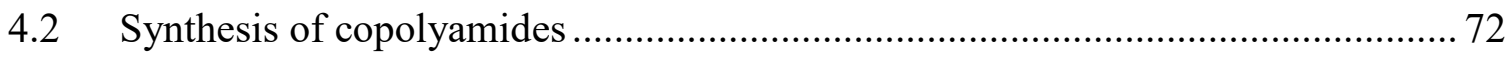

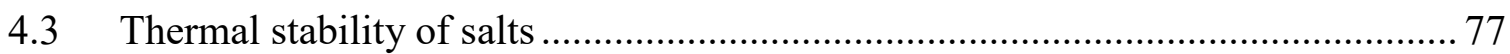

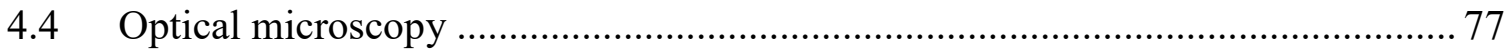

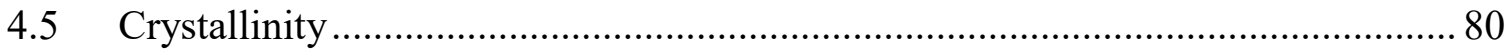

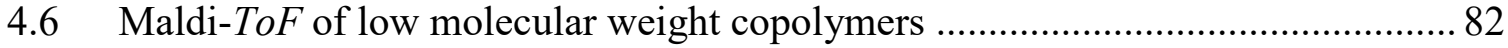

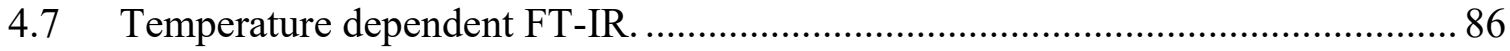

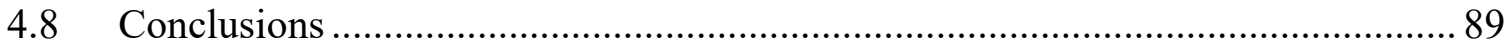

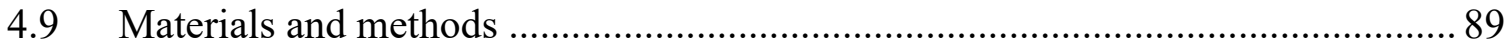

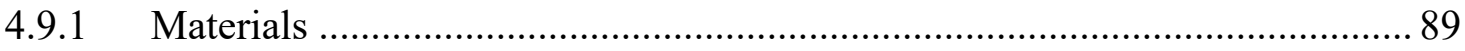

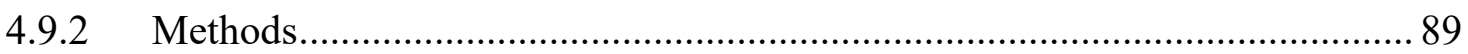

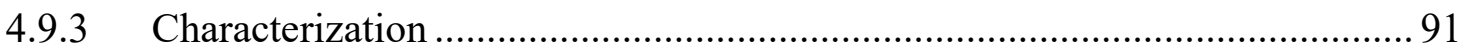

Chapter 5 Thermal stability of GalX during polymerization ..................................... 95

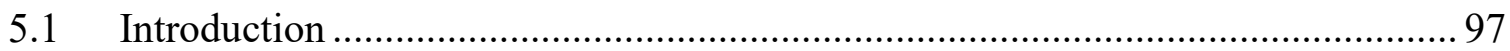

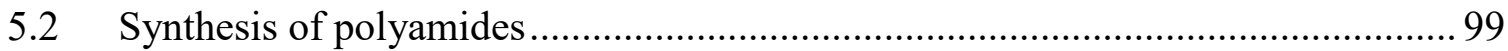

5.2.1 Understanding of the processes occurring during salt polymerization ......... 100 
5.2.2 Polymerization via ester-amide exchange.............................................. 103

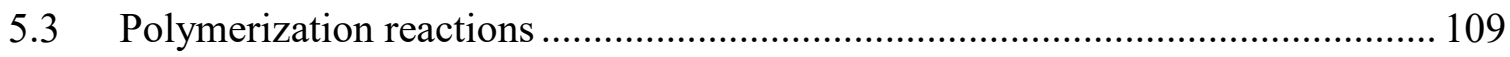

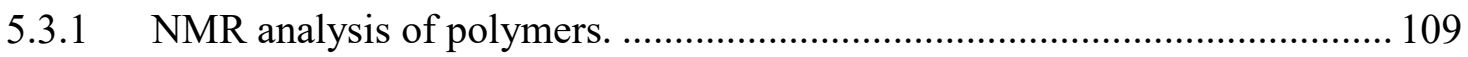

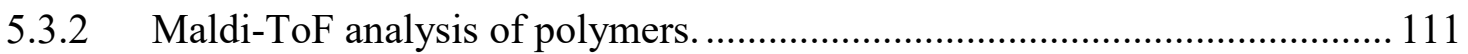

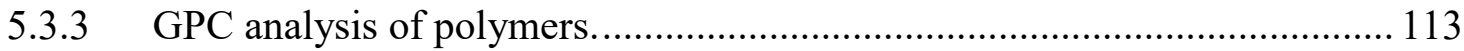

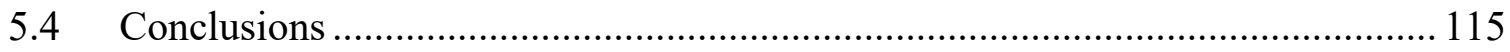

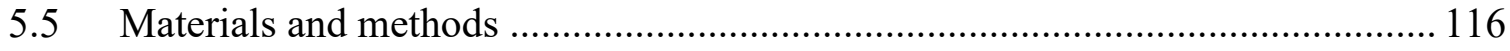

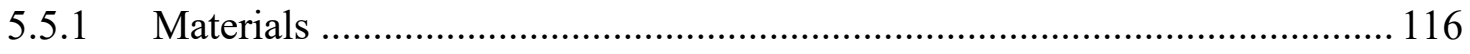

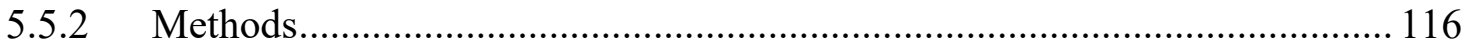

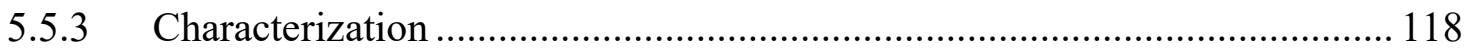

Chapter $6 \quad$ Dioxolane moieties and their interactions........................................... 121

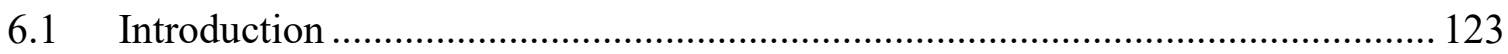

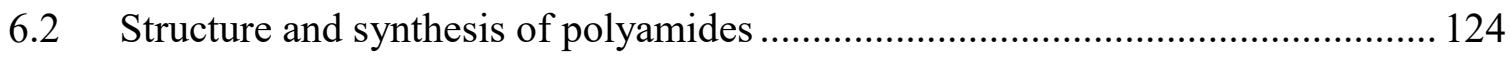

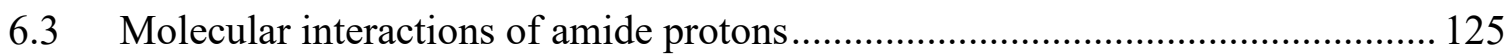

6.4 Stabilization of GalX amide conformations by the formation of intramolecular

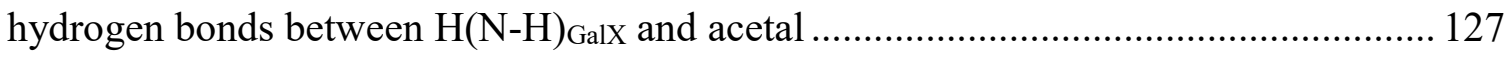

6.5 Distribution of GalX between the amorphous and crystalline phase of copolymers 130

6.6 Hydrogen bonding efficiency

6.7 Melting behavior and Wide-Angle X-ray Diffraction..................................... 133

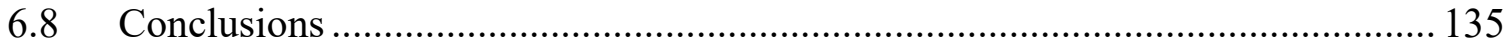

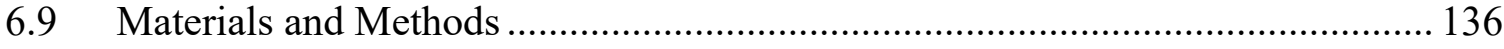

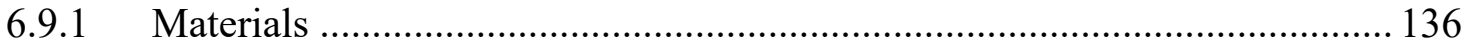

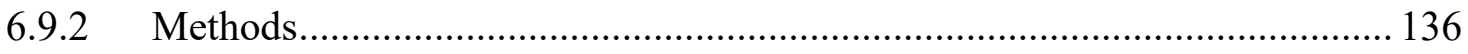

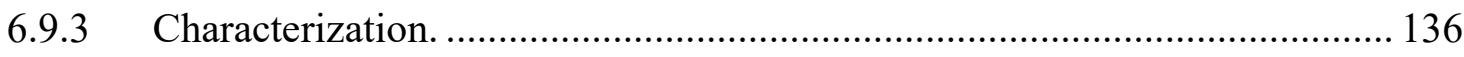

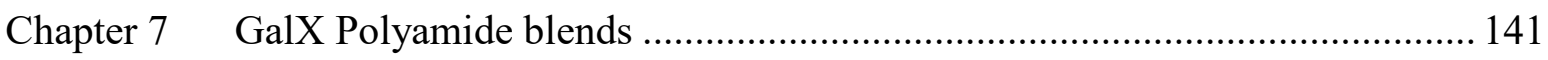

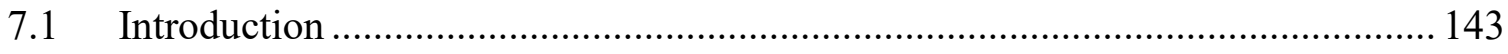




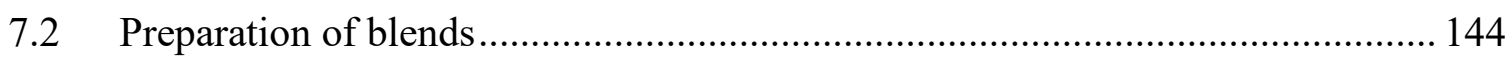

7.3 Thermal and morphological characterization of blends .................................. 146

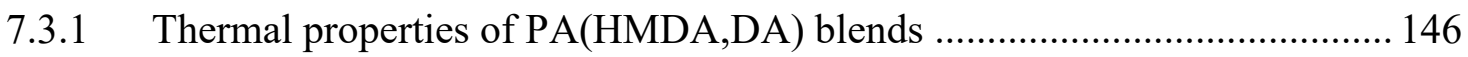

7.3.2 Thermal properties of PA(PACM,DA) blends. ........................................... 146

7.3.3 Morphological study of the blends by SEM. ............................................ 148

7.3.4 Structural transformations induced by thermal processing of

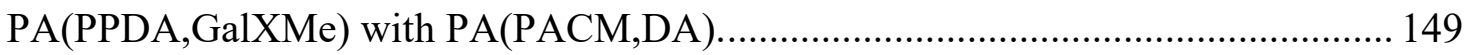

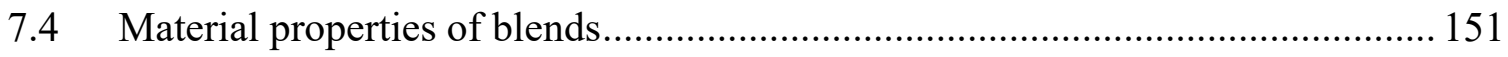

7.4.1 Mechanical performance of blends........................................................ 151

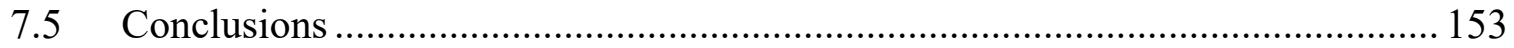

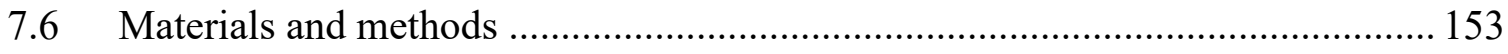

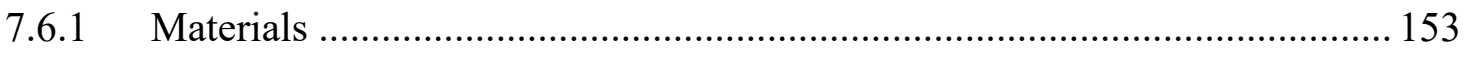

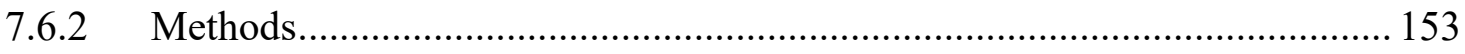

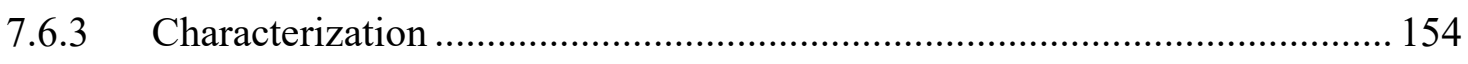

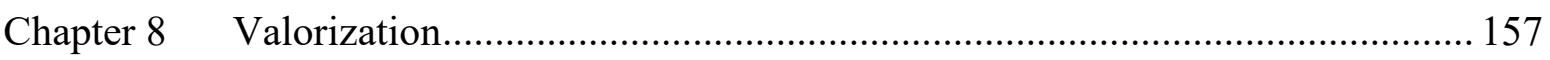

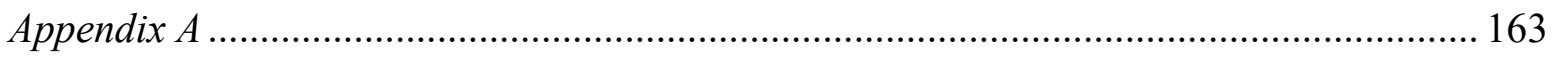

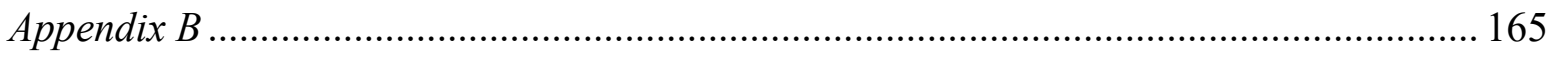

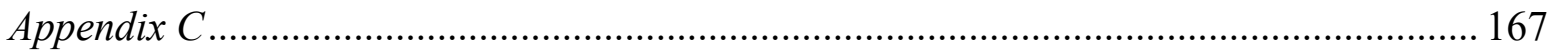

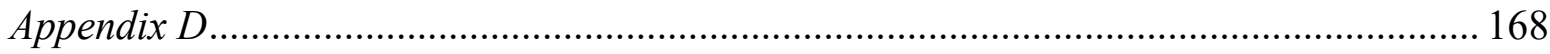

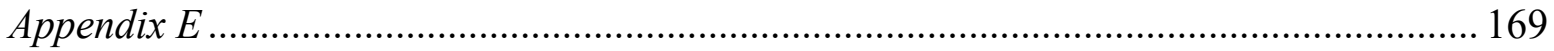

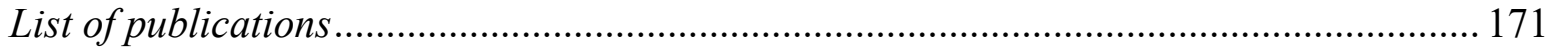

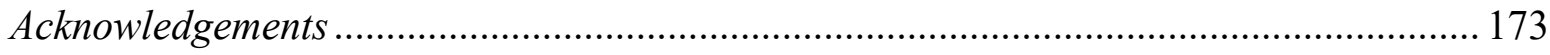

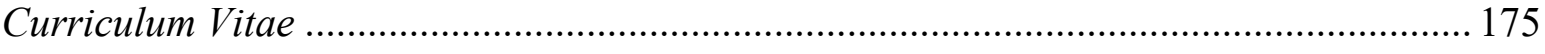




\section{SUMMARY}

Carbohydrates are a widespread class of compounds, which are recognized for their nutritious values and, more recently, as an alternative source of chemicals. In the time when the world struggles with different environmental challenges, exploration of biomass becomes of increasing socio-economical value for the world population.

Polymers (Greek: poly-many, mer - parts) are macromolecules, which are composed of many repeating units and therefore possess unique properties. Polymers "shape" the world as we know it today. Plastic bags, food packaging, plastic in medicine, in cars, at homes - society consumes vast amounts of plastic items and simultaneously generates piles of plastic waste, which is - in most cases - still non bio-based and/or non-biodegradable. ${ }^{1}$ From the early XX century, when first synthetic polymers were intentionally produced, this class of materials developed an extraordinary career. Polyamides, wider known as nylons, share a big part of this popularity. Those synthetic polymers contain peptide bond linkages between the repeating units.

The presented study combines the abovementioned points, focusing on the synthesis of polyamides from carbohydrate-derived building blocks. Specifically, cyclic derivatives of galactaric acid (GalX) are used to prepare bio-based polyamides. The starting molecules are obtained by the extraction and chemical modification of sugar beet pulp, which is, in fact, a waste product generated along the sugar production. The production of galactaric acid does not compete with the production of food and sugar beet can be locally sourced to support local economies.

The polymerization of GalX results in amorphous or semicrystalline polymers with elevated glass transition temperatures in comparison to aliphatic, linear analogues. The crystallinity of polymers can be highly suppressed with increased amount of incorporated GalX. The homopolymers composed of equimolar ratio of diamine and GalX are amorphous. Depending on the rigidity of the diamine, the glass transition temperature $\left(T_{\mathrm{g}}\right)$ of such polymers can be tuned from 50 to $220^{\circ} \mathrm{C}$. The reactivity of the different biacetalized monomers towards the diamines greatly depends on the chemical variation of the $\alpha$ carbon substituent. Compared to non-substituted linear monomer, increased reactivity was observed for different biacetal derivatives of galactaric acid. The polymers derived from the modified galactaric acid monomers vary in terms of glass transition temperature, thermal stability, hydrophilicity and 
functionality. The utilization of carbohydrate-based monomers for the synthesis of polyamides at high temperature can be challenging due to potentially limited stability of the monomers. Investigation of the chemical structures and the analysis of the side reactions helps to reduce effectively the undesired degradation-related phenomena. When the diacid derivative of GalX is polymerized the internally catalyzed deprotection of acetal leads to cross-linked and/or degraded product. The utilization of diethyl esters of GalX usually eliminates the hydrolysis, but leads to the alkylation of amines, which, in consequence, lowers the molecular weight of polymers therefrom.

The properties of polyamides greatly depend on an interaction between amide protons and the oxygen present in the acyl group. In the acetals of galactaric acid other moieties are present which also possess oxygen. The interactions between amide protons, usually occurring in polyamides, are interfered and the polymers have different properties. Furthermore, cyclic moieties are considerably bulky. Consequently, the incorporation of GalX into aliphatic polyamides decreases the density of hydrogen bonding and suppresses crystallinity.

Once synthesized, GalX polyamides are stable under processing conditions and can withstand exposure to high temperatures. The GalX-based polyamides can be blended with other polyamides to form two-phase systems with increased modulus and lowered elongation at break. The miscibility differs depending on the used polyamides. With aliphatic and aromaticaliphatic GalX polyamides a two phase blend was obtained, however, blending GalX polyamides containing fully aromatic 1,4-phenylenediamine resulted in a one phase system. The occurrence of transamidation between blend components leads to reactive compatibilization. 


\section{REFERENCES}

1. M. van den Oever, K. Molenveld, M. van der Zee and H. Bos, Bio-based and biodegradable plastics - Facts and Figures. Focus on food packaging in the Netherlands, Wageningen Food \& Biobased Research, Wageningen, 2017. 



\section{Chapter 1 INTRODUCTION}

\subsection{Renewable resources - Biomass}

Organic matter - a convenient consequence of life on Earth - is used in a variety of applications; it can be converted into energy ${ }^{1}$ or used as a source of renewable buildingblocks $^{2-8}$ for the production of chemicals ${ }^{5,9,10}$ and plastics. ${ }^{7,11-23}$

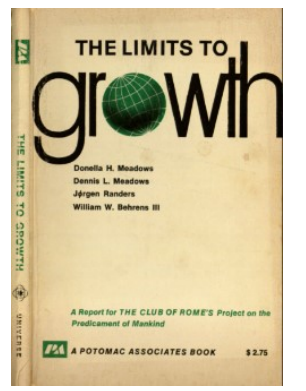

https://upload.wikimedia.org/

A growing interest in renewable energy and resources was started all over the world since economists and researchers started sending alarming messages regarding the depletion of oil and dangerously elevated $\mathrm{CO}_{2}$ emissions. In 1972 the first report The limits to growth ${ }^{24}$ published by the noble organization The Club of Rome ${ }^{25}-$ specialized in the humanitarian challenges - stimulated a global discussion regarding the economic development and its limits, tackling issues like the depletion of the available resources. This discussion, which began almost 50 years ago, is still open with problems highlighted in the report not solved yet, but the search for biobased alternatives to fossil-based products is certainly one of the many angles from which the issue can be addressed. From this perspective biomass is a promising alternative to depleting oil. In addition, bio-based molecules can offer a variety of new functionalities.

Building blocks obtained from renewable sources can be divided into two groups: "drop-in" building blocks which are structurally identical to the fossil-based analogues commonly used in industry, but made from bio-based resources or new building blocks derived from biomass, which bring about new opportunities with new functionality and performance.

Clearly, biomass is a very broad term, which can be applied to world food reserves as well as to generated waste. In these terms it can be subdivided into several categories namely first, second and third generation biomass. ${ }^{1,26}$ While $1^{\text {st }}$ generation feedstock constitues an excellent source of energy and chemicals, it directly competes with world food production and has impact on its prices. A promising alternative to this feedstock is $2^{\text {nd }}$ generation biomass, which consists of a waste product from food industry (e.g. sugar beet pulp). The relatively immature $3^{\text {rd }}$ generation biomass, mainly converting $\mathrm{CO}_{2}$ into organic molecules, utilizes microalgae and microbacteria farming, exploiting processes like fermentation and anaerobic digestion with natural or engineered photochemical metabolic pathways. 


\subsection{Step-growth polymerization}

The most common classification of polymers, proposed by Carothers in 1929, recognizes two major groups: condensation polymers and addition polymers. ${ }^{27,}{ }^{28}$ Condensation polymers are obtained in a step-growth polymerization, which is a polymerization method utilizing a condensation reaction between bifunctional monomers with simultaneous release of a low molecular weight by-product (see Figure 1-1). Typically, in a polycondensation reaction, two different functional groups are able to react with one another (e.g alcohol (optionally amine)carboxylic acid, alcohol (optionally amine) - ester or isocyanates-alcohol yielding polymer). When two reactive groups are present on two different molecules, the resulting monomer is called an AA-BB-type monomer. Alternatively, the two reactive groups can be also present in one molecule, giving an AB-type monomer (e.g. amino acids). In Principles of Polymer Chemistry by Prof. Paul J. Flory ${ }^{28}$ polymers are referred to as polymeric substances which contain a single repeating unit. Condensation polymers are defined as polymeric materials containing the structural unit, which lacks some atoms present in the monomer (“...from which it is formed, or to which it may be degraded"). Addition polymers contain a structural unit, which is identical with the monomer. This definition includes polymers based on $A B$ and AA-BB type of monomers. Copolymers, on the other hand, are defined as polymeric materials containing two or more structural units combined in more or less random sequence. The author specifically recommend using the term polymer (instead of copolymer) to polyamides formed from only one diamine and one diacid.

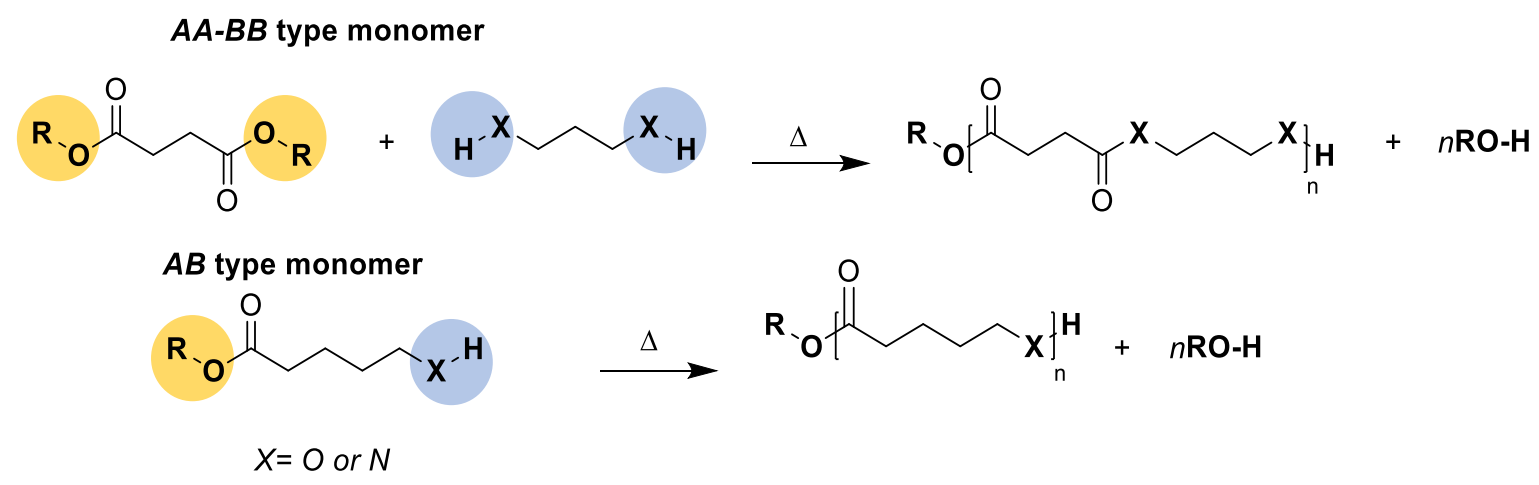

Figure 1-1 General reaction scheme of polycondensation for AA-BB- and AB-type monomers.

Step-growth polymers (polycondensates) are one of the oldest and most useful polymeric materials, with a large variety of potential applications. Examples of such polymers are polyesters, polycarbonates, polyamides, polyurethanes, epoxy resins and polyimides. The pioneer of the step-growth polymerization reaction was Wallace Carothers, who first 
intentionally synthesized polyesters ${ }^{27}$ and later broadened the scope of his research to other synthetic polycondensates. ${ }^{29-31}$

Polycondensates, such as polyesters or polyamides typically reach moderate molecular weights between $10-50 \mathrm{~kg} / \mathrm{mol}^{32}$. On the one hand, the molecular weight of the polymer is directly dependent on the degree of polymerization (DP), a function of conversion $(p)$, which can be influenced by many factors depending on the type of monomers (average functionality $f_{a v}$ ) (see Figure 1-2).

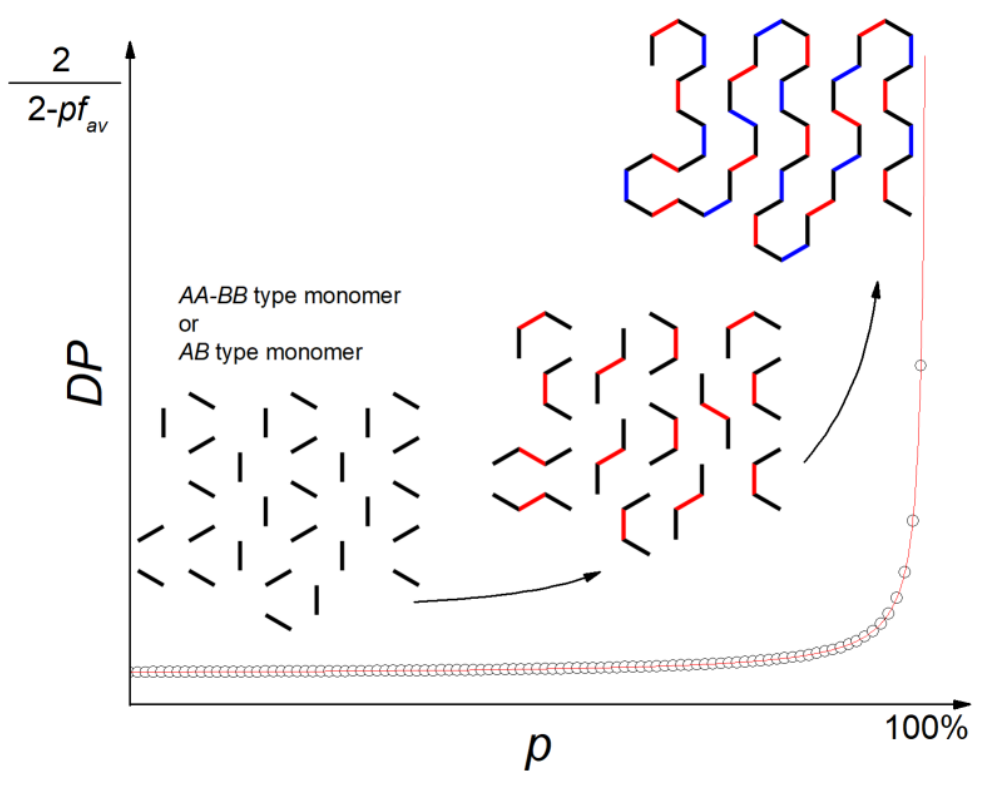

Figure 1- 2 Degree of polymerization (DP) as a function of the conversion (p) for step-growth polymerization with a representative scheme for $A A-B B$-type monomer.

Sufficient molecular weights can only be achieved at high conversion of the functional groups, which contributes to the particular sensitivity of this type of polymerization to stoichiometric imbalance. Most of the monomers, bio-based or traditional oil-based, are prone to sublimate at elevated temperatures, which is challenging during the synthesis. Side reactions constitute another factor, which highly influences the conversion of monomers. The most common side reactions accompanying polycondensation are cyclization, self-condensation of monomers and, in case of amylolysis of esters, the alkylation of amines.

The molecular weight of polymers also determines polymer properties, such as transition temperatures, mechanical properties, chemical resistance and functionality (end-group concentration). Lower molecular weight polymers are typically more soluble in organic 


\section{Chapter 1}

solvents, have higher crystallinity, lower transition temperatures and bear a significant number of functionalities at the chain-end. High molecular weight polymers, on the other hand, perform better in terms of their mechanical strength, chemical and thermal resistance.

Polyamides are a type of polycondensate, which bear within their structure C-N amide bonds, also known as peptide bonds. This particularly important connection shows higher resistance to hydrolysis than a C-O ester bonds and the presence of amide hydrogen stimulates selfassembly of polyamides, both natural and synthetic. Additionally, they have the ability to form extended hydrogen bond networks, which contribute to improved mechanical properties. Due to their high thermal resistance, polyamides are used in automotive applications and due to their high mechanical strength they are widely applied in fiber production. Additionally, a significant number of biopolymers are in fact highly organized polyamides (e.g. peptides).

The most commonly used polyamides are those derived from fossil fuels; however, in recent years more attention has been paid to the utilization of "green" monomer alternatives in the production process. A range of bio-based dicarboxylic acids (sebacic acid, brassylic acid) and diamines (1,4-diaminobutane, 1,6-diaminohexane) are now already available from renewable resources, or are announced to become available from such resources, and are being introduced in large-scale production by companies like DSM (EcoPaXX ${ }^{\circledR}$, ForTii ${ }^{\circledR}$ Eco), Arkema (Rilsan $\left.{ }^{\circledR}\right)$ or Evonik (Vestamid ${ }^{\circledR}$ Terra).

\subsection{Sugar beet as a source of monomers utilized in polymer production}

A promising resource of bio-based monomers and their polymeric materials are carbohydrates. They are widely available, but due to the presence of multiple functionalities they cannot be directly utilized in polymerization. Frequently the hydroxyl groups are in one way or another deactivated leading to bifunctional and thus polymerizable molecules. Carbohydrates are widely acknowledged as a source for both, "drop-in" and "new" monomers; however, the focus of this chapter (and research) is mostly on the latter group. Sugar beet pulp exists out of cellulose, hemicellulose and pectine fractions. 


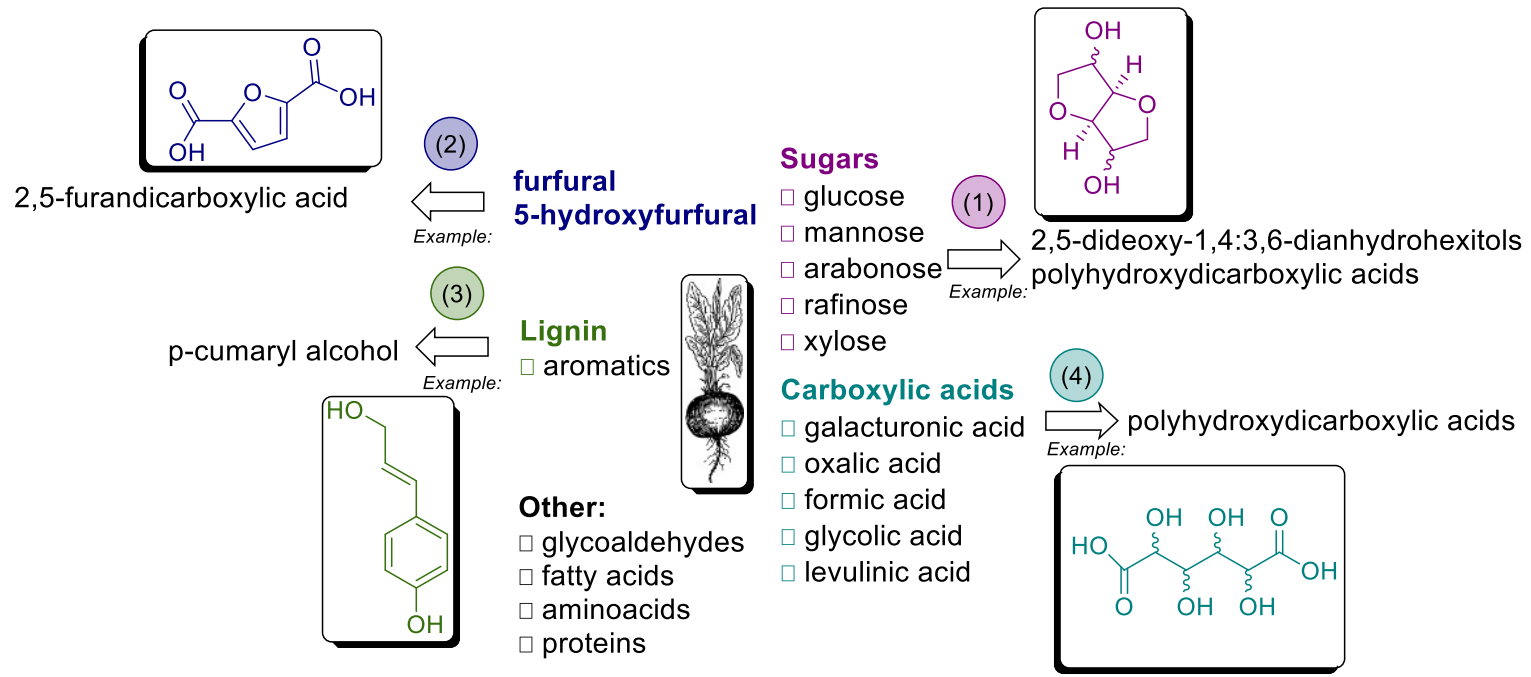

Figure 1-3 Examples of bio-based monomers from sugar beet pulp.

2,5-dideoxy-1,4:3,6-dianhydroalditols (1, Figure 1-3) is a group of diols derived from hexitols by the dehydration of multifunctional sugars. Frequently the monomer preparation is followed by the subsequent transformation of the secondary hydroxyl group to an amine group resulting in 2,5-diamino-1,4:3,6-dianhydroalditols. ${ }^{33-35}$ Due to the presence of two fused rings, the molecules are relatively rigid, contributing to an increase in glass transition temperature and decrease in the melting point of polymers therefrom. The rigidity of the corresponding amines belonging to this monomer family can be countered by the introduction of an ether linkage and longer aliphatic fragments ensuring higher mobility of those fragments e.g. isosorbide(bis(propan-1-amine)) (DAPIS). ${ }^{35}$ Additionally, such moieties can be otherwise functionalized to form dicarboxylic acids e.g. isoidide dicarboxylic acid (IIDCA) ${ }^{36}$, which can be used in the polycondensation process. The introduction of such moieties into aliphatic polymer causes a reduction of hydrogen bonding density, influences crystallinity, transition temperatures and the bicyclic monomer itself is distributed between the amorphous and the crystalline phase of the copolymer.

2,5-furanodicarboxylic acid (2, Figure 1-3) (FDCA) can be obtained from fructose and glucose. ${ }^{7,37}$ It has attracted a lot of attention over the past couple of years, since it provides a unique aromatic structure, and which eventually could replace terephthalic acid. The incorporation of FDCA in polyamide highly influences the hydrogen bonding network as it is prone to form intramolecular hydrogen bonding (Figure 1-4 (a)). ${ }^{38}$ Except this, FDCA molecules have similar thermal properties to terephtalic acid ${ }^{39}$ and are already widely suggested and prepared for the production of plastics e.g. polyesters for plastic bottles as PET 
analogues (PEF) or for the production of engineering plastics, fibers or even bulletproof vests (aramid)..$^{40}$

a.<smiles>CCCNC(=O)c1ccc(C(=O)N([IH])CCC)o1</smiles>

b.<smiles>CCCNC(=O)c1ccc(C(=O)NCCC)cc1</smiles>

Figure 1-4 Structure of amides from a. FDCA and graphical representation of hydrogen bonding typical for polyamides from FDCA, b. terephthalic acid.

Lignin (3, Figure 1-3) is widely recognized as a source of aromatic monomers bearing a whole spectrum of functional groups (e.g.. hydroxyl, diacids or alkene). Similar to 2,5furandicarboxylic acid, it holds great potential for replacing oil as a source of aromatic chemicals with their bio-based alternatives using the products obtained by treatment and hydrolysis of lignin. Most of the obtained products are phenolic replacements to aromatic monomers. The depolymerization of lignin via for example depolymerization is challenging and therefore is now extensively studied in order to optimize the process. ${ }^{41,42}$

Another group of monomers consists of polyhydroxydicarboxylic acids (PHAs) or the corresponding esters (4, Figure 1-3), sourced by the transformation of sugars or by the chemical modification of carboxylic acids extracted directly from sugar beet pulp. Typically, in comparison with other monomers, these compounds contain functionalities in their backbone, such as hydroxyl groups. The carbohydrate-derived molecules may be polymerized directly using sophisticated methods like activation chemistry, condensation of the corresponding dialkyl esters in solution or ring-opening polymerization of lactones formed from linear PHAs in a methanolic solution ${ }^{43-49}$, or alternatively their hydroxyl functionalities can be protected or further functionalized by acetal/ether formation prior to the polymerization process. This group of monomers was extensively studied regarding the preparation of polyester $^{14,16,22,50-54}$, polyamides ${ }^{19,55-60}$, poly(ester-amides $)^{61,62}$ and polyurethanes. $^{15,63}$ 


\subsection{Biacetalized galactaric acid derivatives}

A particular type of O-protected polyhydroxydicarboxylic acids, which can be derived from the pectin fraction in sugar beet pulp, are their acetalized derivatives, which bear within their structure cyclic moieties. Depending on the starting stereoisomer different types of moieties are obtained: ManX and GluX give acetals with two fused 1,3-dioxane rings and GalX gives two 1,3-dioxolane rings separated by a C-C bond. In both, cyclic and non-cyclic polyhydroxydicaboxylic acid functional groups are present in a side chain. Next to the polymers obtained from dicarboxylic acids (or their diesters), the preparation of polymers from different diols of GalX, ManX and GluX is reported. ${ }^{54,63,64}$<smiles>O=C(O)[C@H]1OCO[C@@H]1[C@H]1OCO[C@@H]1C(=O)O</smiles>

GalX-acid<smiles>O=C(O)[C@H]1OCO[C@@H]2[C@@H]1OCO[C@H]2C(=O)O</smiles>

GluX-acid<smiles>O=C(O)[C@H]1OCO[C@@H]2[C@@H]1OCO[C@H]2C(=O)O</smiles>

ManX-acid

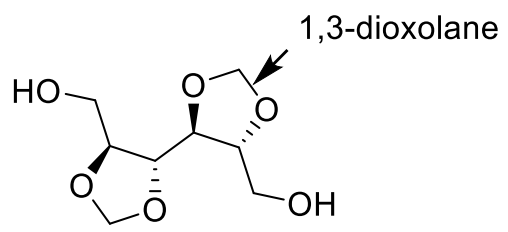

GalX-ol<smiles>OC[C@@H]1OCO[C@@H]2[C@@H](CO)OCO[C@H]12</smiles><smiles>OC[C@@H]1OCO[C@H]2[C@H]1OCO[C@@H]2CO</smiles>

ManX-ol

Figure 1-5 Biacetalized derivatives of galacto-(GalX), D-gluco- (GluX), D-manno-(ManX) sugar derivatives

These biacetalized compounds suppress the crystallinity of the polymers, contributing to an increase in glass transition region and decrease in melting point. In general, the more bulky and rigid the monomer, the more pronounced effects are obtained. The presence of acetals as pendant groups affects the thermal stability of the monomers and therefore delivers new synthethic challenges. So far, the presented moieties are rather utilized for polyester synthesis than for polyamides, since the temperature range during the polymerization is lower.

Galactaric acid used in this study is obtained from the pectin fraction in sugar beet pulp, which is $2^{\text {nd }}$ generation biomass and therefore its production does not compete with the food industry. Even more, it constitutes a potential valorization outlet for the current waste stream 


\section{Chapter 1}

from sugar beets. First, sugar beets are processed in order to obtain raw juice, which is filtered yielding sugar beet pulp as a waste product, besides the purified juice itself, which is used for further processing to obtain white sugar. The sugar beet pulp undergoes thermal, mechanical and physical treatment and after hydrolysis versatile sugar-derived compounds are obtained including galacturonic acid $\mathbf{1}$ - a substrate used for GalX production (see Figure 1-6).

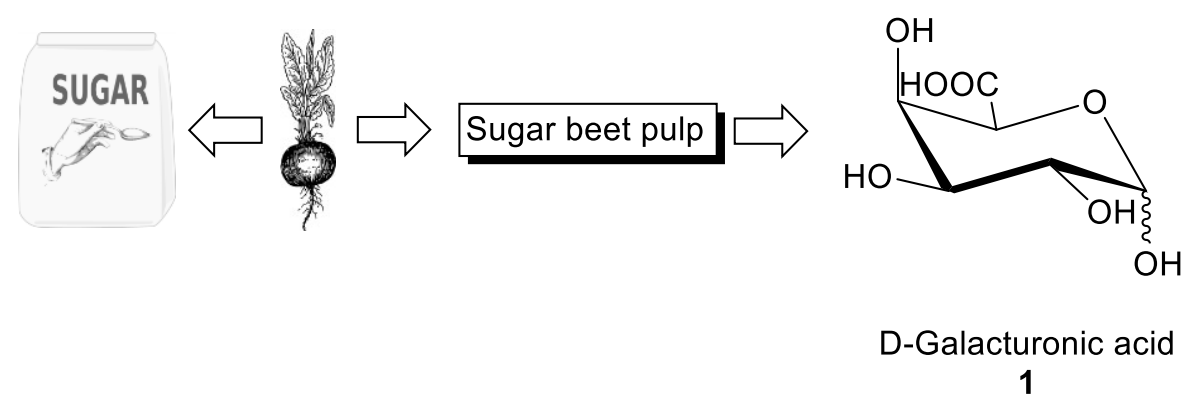

Figure 1-6 The products obtained from sugar beets (Source Royal Cosun).

In order to obtain a bifunctionalized monomer, galacturonic acid $\mathbf{1}$ is oxidized to galactaric acid $2^{65}$ and esterified, yielding diesters of galactaric acid 3 (Figure 1-7). The oxidation process is performed according to a novel procedure using a gold catalyst supported on e.g. $\mathrm{TiO}_{2}$, carbon etc. (see Figure 1-8). In contrast to traditional oxidation with nitric acid this method transforms uronic acids (e.g. galacturonic acid) to aldaric acids (galactaric acid), which does not require double oxidation of both, aldehyde and terminal hydroxyl groups and therefore the side reactions are limited and yields of the oxidation are improved. ${ }^{65,66}$ 
<smiles>COC(=O)[C@H](O)C(O)[C@@H](O)C(=O)OC</smiles>

Diemethyl galactarate (Gal-OMe)

$3 a$<smiles>[R]OC(=O)[C@@H]1OCO[C@H]1[C@H]1OCO[C@H]1C(=O)O[R]</smiles>

dialkyl 2,3;4,5-di-Omethylene galactarate (GalXH-OR)

$4 a$

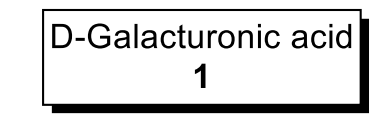

$\left.\begin{array}{l}\text { Catalytic } \\ \text { oxidation }\end{array}\right] \mathrm{y}=95 \%$<smiles>O=C(O)[C@H](O)[C@@H](O)[C@@H](O)[C@@H](O)C(=O)O</smiles>

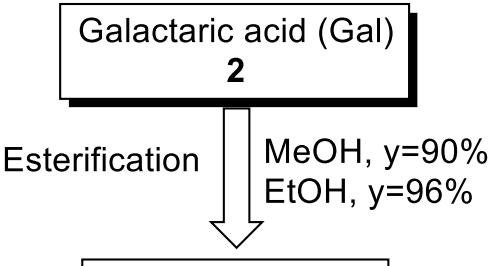

Dialkyl galactarate 3<smiles>CCOC(=O)[C@H](O)[C@@H](O)[C@H](O)[C@@H](O)C(=O)OCC</smiles>

Diethyl galactarate

(Gal-OEt)

3b

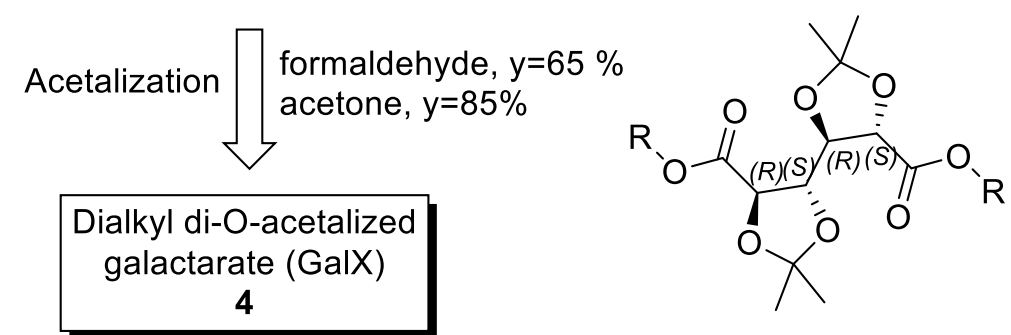

$R=M e / E t$ dialkyl 2,3;4,5-di-Oisopropylidene galactarate (GalXH-OR)

4b

Figure 1-7 The chemical pathways to obtain dialkyl di-O-acetalized galactarates (source Cosun Royal).

The next step, acetalization, is performed in acidic media and results in O-methylene 4a and O-propylidene $\mathbf{4 b}$ acetals of galactarate depending whether formaldehyde or acetone is used in the process. The yield of the reaction varies for different acetalization agents, but is higher for acetone reaching $85 \%, 20 \%$ more than for the analogous reaction with formaldehyde. 
a.

$$
\begin{array}{|c|c|}
\hline \multicolumn{2}{|c|}{\mathrm{CHO}} \\
\mathrm{H} & \mathrm{OH} \\
\mathrm{HO} & \mathrm{H} \\
\mathrm{HO} & \mathrm{H} \\
\mathrm{H} & \mathrm{OH} \\
\hline & \mathrm{CH}_{2} \mathrm{OH} \\
\hline
\end{array}
$$

D-galactose

b.<smiles>O=C(O)C(O)C(O)C(O)C(O)C1CCC1</smiles>

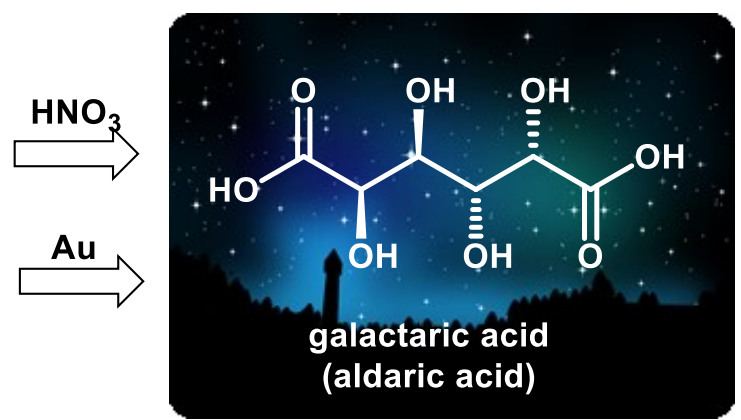

https://openclipart.org

\section{D-galacturonic acid (uronic acid)}

Figure 1-8 A comparison between a. traditional and b. novel method of preparation of galactaric acid.

Optionally, bi-O-acetalized GalX molecules can be hydrolyzed to obtain the free acid $\mathbf{5}$ or reduced, using $\mathrm{LiAlH}_{4}$ to galactitol 6 (Figure 1-9). Hydrolysis is relatively inexpensive, using aqueous $\mathrm{NaOH}$, with the reaction resulting in high yields. The product of the reduced GalXol can be utilized for polyurethanes and polyester synthesis. Another recently proposed functionalization method is modification with aliphatic amines containing other functionalities e.g. thiol or alcohol to obtain $7^{67}$ and it is a reasonable alternative to e.g. the reduction of $\mathrm{LiAlH}_{4}$. The reaction readily proceeds at room temperature with limited side reactions and requires inexpensive and relatively less harmful chemicals than reduction. 


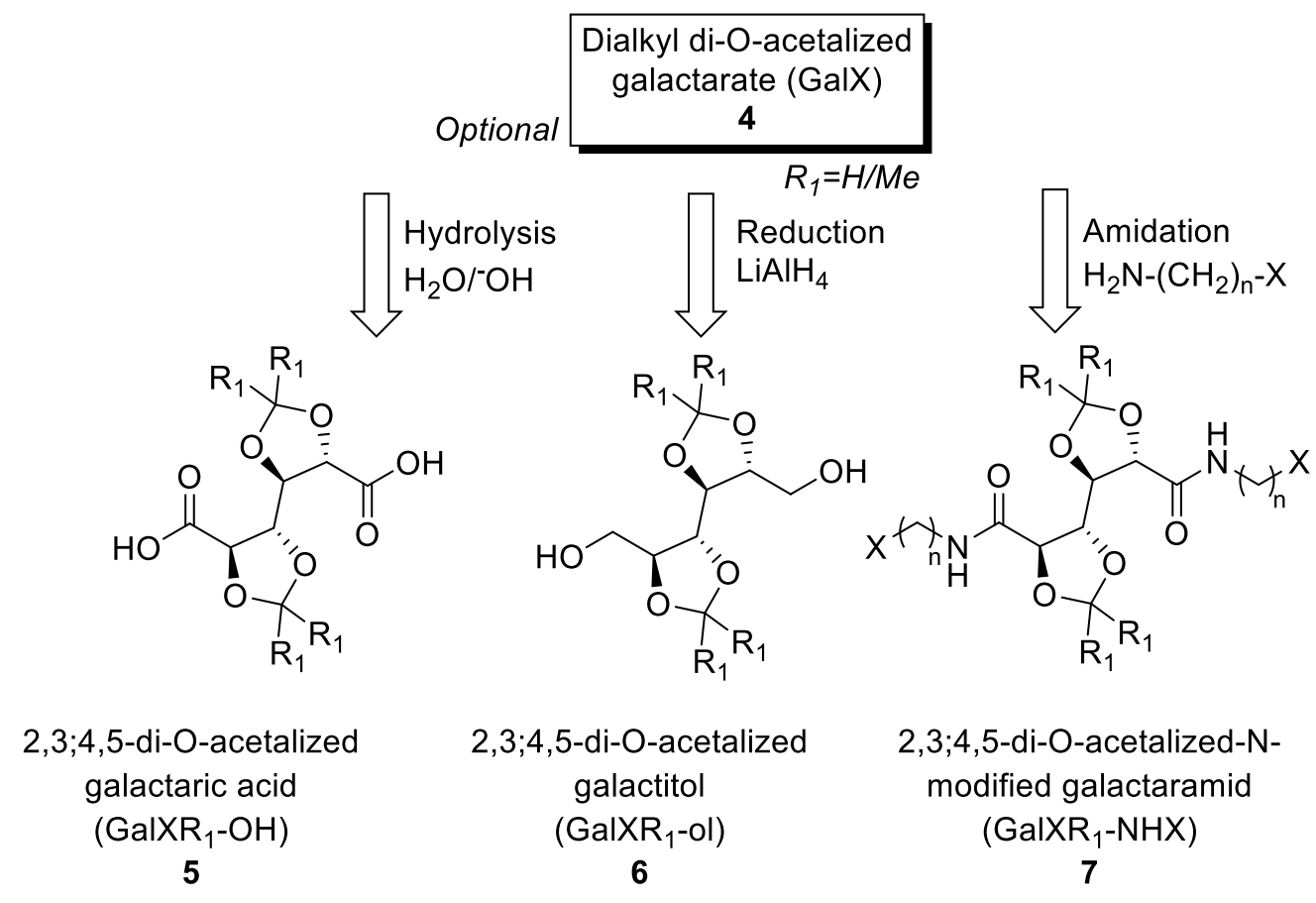

Figure 1-9 The chemical pathways to modify dialkyl di-O-acetalized galactarate (source Cosun Royal)

\subsection{Aim and scope of this study}

Biacetalized galacataric acid derivatives constitute a promising material for polymer synthesis. Bulky and rigid moieties contribute to higher glass transition temperatures, suppressed crystallinity of polymers therefrom and are introducing functionality to polymers. They constitute a good perspective for terephthalic acid replacement or for cyclic monomers utilized in the production of transparent polymers. So far, galactaric acid derivatives were mostly used to obtain polyesters. In the majority of the previous reports concerning polyamides, solution synthetic methods have been used which are not considered as affordable, nor environmentally-friendly, due to the use of organic solvents and toxic chemicals for activation chemistry. ${ }^{10,46}$ In this research homo- and copolyamides of 2,3;4,5di-O-methylene and 2,3;4,5-di-O-isopropylidene galactaric esters $(\mathbf{4 a}, \mathbf{4 b})$ and acids (5) will be investigated. The synthetic approach, properties of the monomers and polyamides made therefrom, as well as their thermal processing will be examined, with a critical assessment of the technological elements involved. 
Chapter 1

\subsection{The outline of the thesis}

The synthesis of amorphous homopolyamides containing diamine and cyclic moieties will be the topic of Chapter 2.

The influence of the protection group on the reactivity of monomers and on the properties of polymers therefrom will be evaluated in Chapter 3.

The content of Chapter 4 includes the preparation of fully and partially bio-based copolyamides via a modified melt polycondensation procedure.

Chapter 5 provides detailed characterization of side reactions occurring during polymerization of 2,3;4,5-di-O-methylene and 2,3;4,5-di-O-isopropylidene galactaric acids and esters.

The inter- and intramolecular (chain) interactions in bio-based polyamides containing cyclic moieties are investigated in Chapter 6.

The thermal processing of polyamides and mechanical properties of polyamide blends are the content of Chapter 7.

Chapter 8 contains the valorization of this research. 


\section{REFERENCES}

1. C. M. Kshirsagar and R. Anand, Appl. Mech. Mater., 2014, 592-594, 1881-1885.

2. A. Behr, J. Eilting, K. Irawadi, J. Leschinski and F. Lindner, Green Chem., 2008, 10, 13-30.

3. P. Wessig and K. Mollnitz, J. Org. Chem., 2012, 77, 3907-3920.

4. M. van Leeuwen, L. Gootjes, W. Vogelzang, R. Knoop, J. van Haveren and D. S. van Es, From Beet Pulp To Building Blocks and Polymers Developing Value Added Materials From GalX, http://edepot.wur.nl/405734, (accessed 03 October 2017, 2017).

5. S. Thiyagarajan, L. Gootjes, W. Vogelzang, J. van Haveren, M. Lutz and D. S. van Es, ChemSusChem, 2011, 4, 1823-1829.

6. L. Grazia, D. Bonincontro, A. Lolli, T. Tabanelli, C. Lucarelli, S. Albonetti and F. Cavani, Green Chem., 2017, 19, 4412-4422.

7. Y. Jiang, D. Maniar, A. J. Woortman, G. O. Alberda van Ekenstein and K. Loos, Biomacromolecules, 2015, 16, 3674-3685.

8. M. Cao, C. Zhang, B. He, M. Huang and S. Jiang, Macromol. Res., 2017, 25, 722-729.

9. M. Pelckmans, T. Renders, S. Van de Vyver and B. F. Sels, Green Chem., 2017, 19, 53035331

10. M. Mancera, I. Roffé, M. Rivas and J. A. Galbis, Carbohydr. Res., 2003, 338, 1115-1119.

11. I.-C. Yeh, B. C. Rinderspacher, J. W. Andzelm, L. T. Cureton and J. La Scala, Polymer, 2014, 55, 166-174.

12. Y. Jiang, D. Maniar, A. J. J. Woortman and K. Loos, RSC Adv., 2016, 6, 67941-67953.

13. Y. K. Endah, S. H. Han, J. H. Kim, N.-K. Kim, W. N. Kim, H.-S. Lee and H. Lee, J. Appl. Polym. Sci., 2016, 133, n/a-n/a.

14. C. Lavilla, A. Alla, A. M. de Ilarduya, E. Benito, M. G. Garcia-Martin, J. A. Galbis and S. Munoz-Guerra, Biomacromolecules, 2011, 12, 2642-2652.

15. C. Hahn, H. Keul and M. Möller, Polym. Int., 2012, 61, 1048-1060.

16. C. Lavilla and S. Muñoz-Guerra, Green Chem., 2013, 15, 144-151.

17. P. B. Smith, in Green Polymer Chemistry: Biobased Materials and Biocatalysis, American Chemical Society, 2015, ch. 27, pp. 453-469.

18. A. Wroblewska, S. Lingier, J. Noordijk, F. E. Du Prez, S. M. A. Wildeman and K. V. Bernaerts, Eur. Polym. J., 2017, 96, 221-231.

19. A. A. Wróblewska, K. V. Bernaerts and S. M. A. De Wildeman, Polymer, 2017, 124, $252-$ 262.

20. T. Mehtio, L. Nurmi, V. Ramo, H. Mikkonen and A. Harlin, Carbohydr. Res., 2015, 402, 102-110.

21. F. Fenouillot, A. Rousseau, G. Colomines, R. Saint-Loup and J. P. Pascault, Prog. Polym. Sci., 2010, 35, 578-622.

22. E. Zakharova, A. Martínez de Ilarduya, S. Leon and S. Muñoz-Guerra, Des. Monomers Polym., 2017, 20, 157-166.

23. J. A. Galbis, M. de Gracia Garcia-Martin, M. V. de Paz and E. Galbis, Chem. Rev., 2016, 116, $1600-1636$.

24. D. H. Meadows, D. L. Meadows, J. Randers and W. W. Behrens III, The limits to growth, Potomac Associates - Universe Books, New York, 1972.

25. Wikipedia, https://en.wikipedia.org/wiki/Club of Rome, (accessed 26.01.2018).

26. K. Srirangan, L. Akawib, M. Moo-Younga and C. P. Choua, Appl. Energy, 2012, 100, 172186.

27. W. H. Carothers and J. A. Arrvin, J. Am. Chem. Soc., 1929, 51, 2560-2570.

28. Flory; P.J., Principles of Polymer Chemistry Cornell University Press, Ithaca and London, 1986.

29. W. H. Carothers and G. J. Berchet, J. Am. Chem. Soc., 1930, 52, 5289-5291.

30. J. W. Hill and W. H. Carothers, J. Am. Chem. Soc., 1932, 54, 1569-1579.

31. J. W. Hill and W. H. Carothers, J. Am. Chem. Soc., 1932, 54, 1579-1587.

32. M. E. Rogers and T. E. Long, Synthetic methods in step-growth polymers., A John Wiley \& Sons, inc., Hoboken, New Jersey, 2003. 
33. J. Wu, L. Jasinska-Walc, D. Dudenko, A. Rozanski, M. R. Hansen, D. van Es and C. E. Koning, Macromolecules, 2012, 45, 9333-9346.

34. L. Jasinska, M. Villani, J. Wu, D. van Es, E. Klop, S. Rastogi and C. E. Koning, Macromolecules, 2011, 44, 3458-3466.

35. A. Wroblewska, A. Zych, S. Thiyagarajan, D. Dudenko, D. van Es, M. R. Hansen, C. Koning, R. Duchateau and L. Jasinska-Walc, Polym. Chem., 2015, 6, 4133-4143.

36. L. Jasinska-Walc, D. Dudenko, A. Rozanski, S. Thiyagarajan, P. Sowinski, D. van Es, J. Shu, M. R. Hansen and C. E. Koning, Macromolecules, 2012, 45, 5653-5666.

37. J. Tomaszewska, D. Bielinski, M. Binczarski, J. Berlowska, P. Dziugan, J. Piotrowski, A. Stanishevsky and I. A. Witonska, RSC Advances, 2018, 8, 3161-3177.

38. C. H. R. M. Wilsens, Y. S. Deshmukh, B. A. J. Noordover and S. Rastogi, Macromolecules, 2014, 47, 6196-6206.

39. M. Winnacker and B. Rieger, Macromol. Rapid Commun., 2016, 37, 1391-1413.

40. M. Acemoglu, S. Bantle, T. Mindt and F. Nimmerfall, Macromolecules, 1995, 28, 3030-3037.

41. S. Gillet, M. Aguedo, L. Petitjean, A. R. C. Morais, A. M. da Costa Lopes, R. M. Lukasik and P. T. Anastas, Green Chem., 2017, 19, 4200-4233.

42. Z. Sun, B. Fridrich, A. de Santi, S. Elangovan and K. Barta, Chem. Rev., 2018, 118, 614-678.

43. D. E. Kiely, A. Vishwanathan, B. P. Jarman and M. Manley-Harris, J. Carbohydr. Chem., 2009, 28, 348-368.

44. B. P. Jarman, D. E. Kiely, M. Manley-Harris and B. K. Nicholson, J. Carbohydr. Chem., 2009, 28, 107-123.

45. N. Ogata, K. Sanui, K. Hosoda and H. Nakamura, J. Polym. Sci., Polym. Chem. Ed., 1976, 14, 783-792.

46. N. Ogata, K. Sanui, K. Hosoda and H. Nakamura, J. Polym. Sci., Polym. Chem. Ed., 1977, 15, 1523-1526.

47. N. Ogata, K. Sanui, H. Nakamura and H. Kishi, J. Polym. Sci., Polym. Chem. Ed., 1980, 18, 933-938.

48. N. Ogata, K. Sanui, T. Ohtake and H. Nakamura, Polym. J., 1979, 11, 827-833.

49. D. E. Kiely, L. Chen and T. H. Lin, J. Am. Chem. Soc., 1994, 116, 571-578.

50. C. Lavilla, A. Alla, A. Martínez de Ilarduya, E. Benito, M. G. García-Martín, J. A. Galbis and S. Muñoz-Guerra, J. Polym. Sci., Part A: Polym. Chem., 2012, 50, 3393-3406.

51. C. Lavilla, A. Alla, A. Martínez de Ilarduya, E. Benito, M. G. García-Martín, J. A. Galbis and S. Muñoz-Guerra, J. Polym. Sci., Part A: Polym. Chem., 2012, 50, 1591-1604.

52. E. Gubbels, C. Lavilla, A. M. de Ilarduya, B. A. J. Noordover, C. E. Koning and S. MuñozGuerra, J. Polym. Sci., Part A: Polym. Chem., 2014, 52, 164-177.

53. S. Muñoz-Guerra, C. Lavilla, C. Japu and A. Martínez de Ilarduya, Green Chem., 2014, 16, 1716-1739.

54. C. Lavilla, E. Gubbels, A. Martínez de Ilarduya, B. A. J. Noordover, C. E. Koning and S. Muñoz-Guerra, Macromolecules, 2013, 46, 4335-4345.

55. S. Munoz-Guerra, High Perform. Polym., 2012, 24, 9-23.

56. J. I. Iribarren, C. Aleman, J. J. Bou and S. Munoz-Guerra, Macromolecules 1996, 29, $4397-$ 4405.

57. P. Ruiz-Donaire, J. J. Bou, S. Munoz-Guerra and A. Rodriquez-Galan, J. Appl. Polym. Sci., 1995, 58, 41-54.

58. J. J. Bou, A. Rodriquez-Galan and S. Munoz-Guerra, Macromolecules, 1993, 26, 5664-5670.

59. A. Rodriquez-Galan, J. J. Bou and S. Munoz-Guerra, J. Polym. Sci., Part A: Polym. Chem., 1992, 30, 713-721.

60. C. Rosu, I. I. Negulescu, R. Cueto, R. Laine and W. H. Daly, J. Macromol. Sci., Part A Pure Appl. Chem., 2013, 50, 940-952.

61. I. Villuendas, J. I. Iribarren and S. Munoz-Guerra, Macromolecules, 1999, 32, 8015-8023.

62. C. Regano, A. M. De Ilarduya, J. I. Iribarren and S. Munoz-Guerra, J. Polym. Sci., Part A: Polym. Chem., 2000, 38, 2687-2696.

63. T.-M. Chen, Y.-F. Wang, S. Sakamoto, K. Okada and T. Nakaya, Des. Monomers Polym., $1998, \mathbf{1}, 447-465$. 
64. G. Prompers, H. Keul and H. Hocker, Green Chem., 2006, 8, 467-478.

65. D. S. van Es, J. van Haveren, H. Raaijmaakers, F. van der Klis, G. P. F. M. van Engelen and A. E. Frissen, US 9,079,844 B2, 2014.

66. C. G. Sauer, I. Barnes, K. H. Becker, H. Geiger, T. J. Wallington, L. K. Christensen, J. Platz and O. J. Nielsen, J. Phys. Chem. A, 1999, 103, 5959-5966.

67. R. Lazeroms, H. Raaijmaakers, C. E. Koning, A. Papegaai and A. Urnianova, WO 201S/074926 A1, 2018. 



\section{Chapter 2 PROPERTIES OF GALX POLYAMIDES}

Abstract

This chapter focuses on the preparation and characterization of polyamides obtained by the polycondensation of 2,3;4,5-di-O-methylene-galactarate (GalXH) and 2,3:4,5-di-Oisopropylidene-galactarate (GalXMe) and diamines. The diamines vary in terms of the chemical structure. Two different approaches towards the synthesis of bio-based polyamides are evaluated: melt polycondensation of diesters and direct polycondensation in solution via the phosphorylation technique. The polymerization of GalXMe with diamines results in polymers with narrow dispersity whereas GalXH gives polyamides with broad dispersity. The prepared polyamides are amorphous with glass transition temperatures between $50{ }^{\circ} \mathrm{C}-$ $220^{\circ} \mathrm{C}$. The obtained polyamides are stable at elevated temperatures (i.e. above $300^{\circ} \mathrm{C}$ ). The hydrolytic stability of the acetal groups in the GalX polyamides in different acids (e.g. $5 \%$ citric acid, $90 \%$ formic acid) is investigated and reveals higher acid resistance of GalXH compared to that of GalXMe. The structures of the polyamides are elucidated by a combination of NMR, FT-IR and Maldi-ToF techniques. 



\subsection{Introduction}

Over the last two decades, numerous studies concerning the development of bio-based materials have been conducted ${ }^{1,2}$, largely resulting from a continuous demand for new cutting-edge bio-based molecules. This study explores the use of new bio-based building blocks derived from sugar beet pulp (a waste product) for the synthesis of novel polyamide grades with elevated glass transition temperatures $\left(T_{\mathrm{g}}\right)$. More specifically, bifunctional formaldehyde- and acetone-protected galactarate derivatives 2,3;4,5-di- $O$-methylenegalactarate $(\mathrm{GalXH})$ and 2,3:4,5-di-O-isopropylidene-galactarate (GalXMe) are studied. The bicyclic structure of these carbohydrate-based building blocks provides stiffness to the polymer backbone and therefore increases the $T_{\mathrm{g}}$. This behavior was broadly investigated for aromatic and aliphatic (co)polyesters ${ }^{3-6}$ containing 2,3;4,5-di- $O$-methylene-galactarate $(\mathrm{GalXH})$. The insertion of bicyclic carbohydrate units in e.g. poly(terephthalate)s, slightly reduces the thermal stability of the polymer. The bicyclic carbohydrates have a suppressing effect on crystallinity but generally increase the $T_{\mathrm{g}}{ }^{7}$ The $T_{\mathrm{g}}$ of homopolyesters of adipic acid and hexamethylenediol increases from $-61{ }^{\circ} \mathrm{C}$ to $6{ }^{\circ} \mathrm{C}$ upon the replacement of adipic acid with a GalXH unit. ${ }^{5}$ Similarly, the replacement of 1,4-butanediol with the sugar-based diol 2,3:4,5-di- $O$-methylene-galactitol in poly(butylene terephthalate) resulted in a polyester with a $T_{\mathrm{g}}$ almost $60{ }^{\circ} \mathrm{C}$ higher (from $31{ }^{\circ} \mathrm{C}$ to $87{ }^{\circ} \mathrm{C}$ ). ${ }^{8}$ Terephthalate polyesters containing protected galactarate or galactitol are characterized by enhanced sensitivity to degradation in acidic conditions and biodegradability. ${ }^{3,9}$ The configuration of sugar derivatives determines the sort of cyclic acetal formed upon protection. D-manno- and D-gluco- based compounds mostly form acetals based on the 6-membered ring of 1,3-dioxane and the galactaro- unit preferably forms 5-member rings based on the 1,3-dioxolane structure. Although the galactaro-derived polymers are known to have a lower impact on the $T_{\mathrm{g}}$, compared to their D-gluco- or D-manno- equivalents, their increased thermal stability is considered to be beneficial during high temperature polycondensation. ${ }^{8}$

So far little prior work is available concerning galactarate derived polyamides. The first attempts to incorporate protected carbohydrate structures into polymers were already patented in the $1950 \mathrm{~s}{ }^{10}$, where it was stated that linear polyamides with high molecular weight could be obtained, starting from protected galactarate structures using a variety of synthesis methods (e.g. solution/melt polycondensation). Shortly after this patent, another more general patent was released, which dealt with different polyamides and polyesters 


\section{Chapter 2}

obtained from carbohydrates like D-mannitol, D-glucitol, galactitol, D-furanose and galactarates made via interfacial polycondensation. ${ }^{11}$

In 2013, Rosu et al. ${ }^{12}$ described GalXMe polyamides and their characterization with FT-IR, GPC, Maldi-ToF and ${ }^{13} \mathrm{C}$ NMR techniques. Although the authors' aim was to obtain unprotected GalX polymers, it does, nevertheless, provide valuable information about ketalized GalX polyamides. Polymers based on hexamethylenediamine and diethyl 2,3:4,5di-O-isopropylene-galactarate were prepared in solution resulting in low molecular weight polyamides ( $M_{\mathrm{n}}$ between 800 and $3600 \mathrm{~g} / \mathrm{mol}$ determined by GPC), followed by the deprotection of the ketal in $90 \%$ trifluoroacetic acid.

The investigation of stereoregular polyamides from cyclic O-methylene protected tartaric acid, which is in fact half of the GalXH molecule, revealed that the target polyamides are highly crystalline, optically active and easily soluble in chloroform. ${ }^{13}$

In this part the synthesis of polyamides with a high $T_{g}$, via affordable and industrially relevant polycondensation from protected galactarates will be a subject of the study. Such polyamides could, for instance, find application in consumer products like appliances for which often transparent materials are required. The bulky structure of the GalX is expected to suppress crystallinity and the rigidity of the dioxolane fragments to elevate glass transition region.

Several polycondensation methods are described that eventually could also be realized at larger scale, as well as smaller, lab-scale procedures, which in turn allow an understanding of the underlying mechanistic principles (with possible side reactions lowering the molecular weight). The chemical and physical characterization as well as the hydrolytic and thermal stability of the obtained polymeric materials is characterized. 


\subsection{The synthesis and properties of homopolyamides with different diamines}

In order to obtain GalX homopolyamides two different approaches towards polyamide synthesis were investigated. The melt polycondensation of diester and diamines was used to synthesize two series of rigid polyamides based on GalXH and GalXMe (see Figure 2-1).
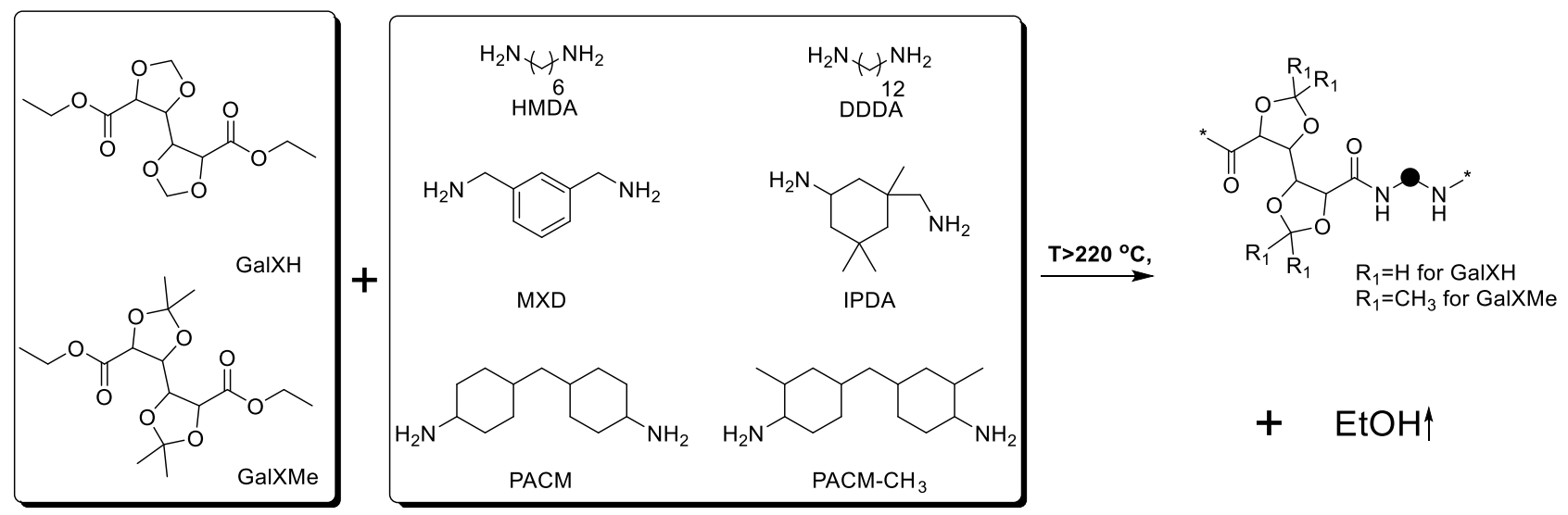

Figure 2-1 The reaction scheme and chemical structures of GalX, diamines and polymer therefrom.

The second investigated approach utilizes direct polycondensation of dicarboxylic acid with aromatic diamines with in-situ formation of triphenyl phosphite esters (Figure 2-2). This technique is recommended especially in the polymerization of diacids with aromatic diamines ${ }^{14}$, since the reactivity of the amine groups attached to the aromatic rings is limited and melt polycondensation results in degradation of the amine.
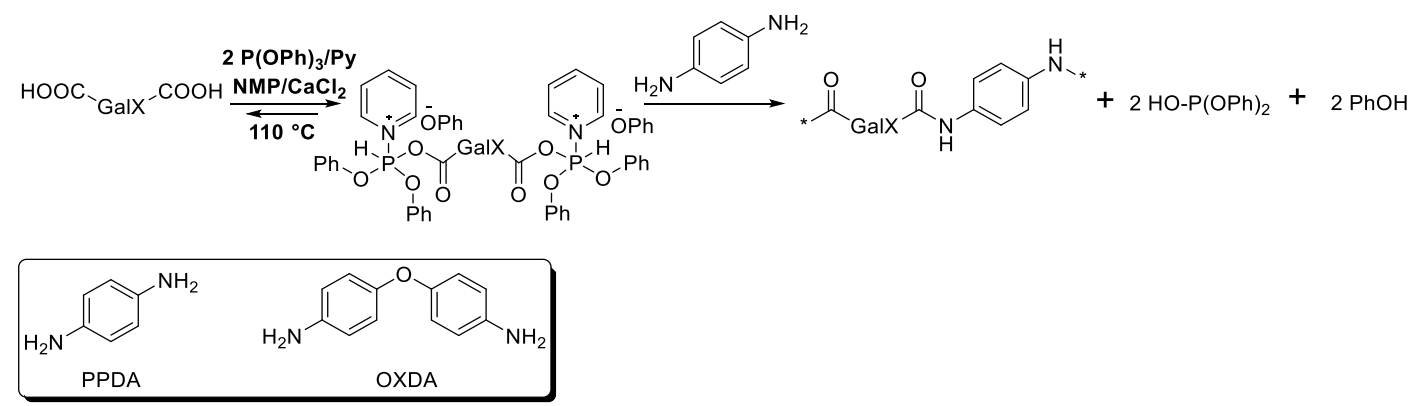

Figure 2-2 Reaction scheme for the Yamazaki-Higashi phosphorylation.

The method itself is effective towards aromatic diamines like p-phenylenediamine (PPDA) or 4,4'-oxydianiline (OXDA); however it does not afford good yields when diacids are combined with aliphatic diamines. The low temperatures of the reaction and activation of the acid groups are favored with respect to the utilization of aromatic moieties; however; the multiple, toxic reagents and solvents used in the course of the experiment are a serious 
drawback with respect to economic and environmental issues. Nonetheless, this method will be applied for aromatic moieties to investigate polyamides with high glass transitions.

Table 2-1 Composition, molecular weights and thermal properties of polyamides with 50/50 GalX/diamine (GalX/DA) molar ratio in the feed.

\begin{tabular}{|c|c|c|c|c|c|c|c|}
\hline $\begin{array}{l}\text { Synthethic } \\
\text { aproach }\end{array}$ & Entry & Diamine & $\begin{array}{l}T_{g}{ }^{a} \\
\left({ }^{\circ} \mathrm{C}\right)\end{array}$ & $\begin{array}{c}M_{n}{ }^{b} \\
\left(\mathrm{~kg}^{\prime} \cdot \mathrm{mol}^{-1}\right)\end{array}$ & $\boldsymbol{D}^{b}$ & $\begin{array}{l}\text { Conv }_{G a l X} \\
\text { /Conv }_{D A}{ }^{c} \\
(\% / \%)\end{array}$ & $\begin{array}{c}\text { Mol \% } \\
\text { GalX/D } A^{c}\end{array}$ \\
\hline \multicolumn{8}{|c|}{ GalXH } \\
\hline \multirow{6}{*}{ 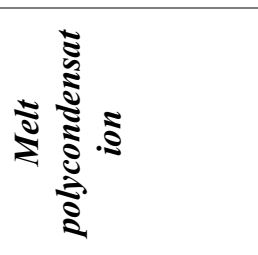 } & PA1 & HMDA & 77 & 24 & 4.32 & $95.6 / 97.6$ & $49.6 / 50.4$ \\
\hline & PA2 & DDDA & 49 & 31 & 5.68 & $97.0 / 98.5$ & $49.5 / 50.5$ \\
\hline & PA3 & MXD & 125 & 20 & 3.38 & $95.9 / 96.3$ & $48.8 / 51.2$ \\
\hline & PA4 & IPDA & 158 & 7 & 1.94 & $90.9 / \mathrm{NA}$ & $54.3 / 45.7$ \\
\hline & $\mathrm{PA}^{\$}$ & PACM & 126 & 3 & 2.60 & $94.8 / 81.1$ & $47.3 / 52.6$ \\
\hline & PA6* $^{*}$ & PPDA & NA & 2.1 & 4.70 & NA & NA \\
\hline \multirow[t]{2}{*}{ Phosphorylation } & PA7 & PPDA & 224 & 17 & 1.90 & NA & $50.2 / 49.8$ \\
\hline & PA8 & OXD & 184 & 6.7 & 2.32 & NA & $50 / 50$ \\
\hline
\end{tabular}

GalXMe

\begin{tabular}{|c|c|c|c|c|c|c|c|}
\hline \multirow{7}{*}{ 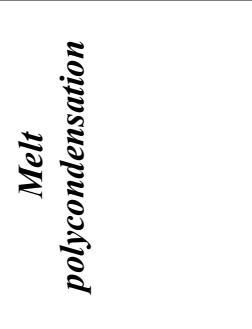 } & PA9 & HMDA & 82 & 23 & 2.50 & $97.7 / 97.3$ & $49.1 / 50.9$ \\
\hline & PA10 & DDDA & 51 & 21 & 2.04 & $99.0 / 97.2$ & $49.0 / 51.0$ \\
\hline & PA11 & MXD & 119 & 19 & 2.16 & $98.7 / 99.8$ & $50.7 / 49.3$ \\
\hline & PA12 & IPDA & 158 & 17 & 1.72 & $97.9 / 98.2$ & $51.7 / 48.3$ \\
\hline & PA13 & PACM & 154 & 10 & 2.13 & $98.9 / 99.0$ & $48.5 / 51.5$ \\
\hline & PA14 & $\begin{array}{l}\text { PACM- } \\
\mathrm{Me}\end{array}$ & 142 & 5 & 1.85 & NA & NA \\
\hline & PA15* & PPDA & NA & 4.5 & 5.94 & NA & NA \\
\hline \multirow[t]{2}{*}{ Phosphorylation } & PA16 & PPDA & 223 & 36 & 2.15 & NA & $50 / 50$ \\
\hline & PA17 & OXD & 199 & 26 & 2.52 & NA & $50 / 50$ \\
\hline
\end{tabular}

*partial degradation of polymer synthesized in melt from aromatic diamines was observed manifested in discoloration of the polymer and high dispersities $\$$ polymer was partially gelled, analytical results for the soluble fraction

${ }^{a}$ determined by DSC ${ }^{b}$ Determined by GPC ${ }^{c}$ ConvGalX/ConvDA $(\% / \%)=\%$ conversion GalX/\% conversion diamine (DA) determined by ${ }^{1} \mathrm{H}$ NMR analysis (see Appendix A)

Table 2-1 shows a list of the polyamides, which were synthesized via the abovementioned methods together with the thermal properties and molecular weight data. Polyamides based on diamines with both amine groups attached to a secondary carbon (see entry PA1-PA3 PA9-PA11) give molecular weights ranging from 19 to $31 \mathrm{~kg} \cdot \mathrm{mol}^{-1}$ while polyamides from diamines with both amine groups attached to a tertiary carbon (PA13, PA14) result in lower molecular weights $\left(3-10 \mathrm{~kg} \cdot \mathrm{mol}^{-1}\right)$. Intermediate molecular weights between 7 and 17 $\mathrm{kg} \cdot \mathrm{mol}^{-1}$ were obtained with the isophorone diamine, which contains one amine group attached to the secondary and one to the tertiary carbon (entry PA4 and PA12). The observed trend in molecular weight is in line with the expectations, since sterically bulky amines connected to tertiary carbons will be weaker nucleophiles than less hindered primary amines. For example, polyamides prepared from cyclic PACM and $\mathrm{PACM}-\mathrm{CH}_{3}$ differ in molecular 
weight significantly (see entry PA13, PA14 in Table 2-1). The presence of the additional methyl group in the proximity of the amine group contributes to increased steric hindrance, which, therefore, lowers the reactivity of this species.
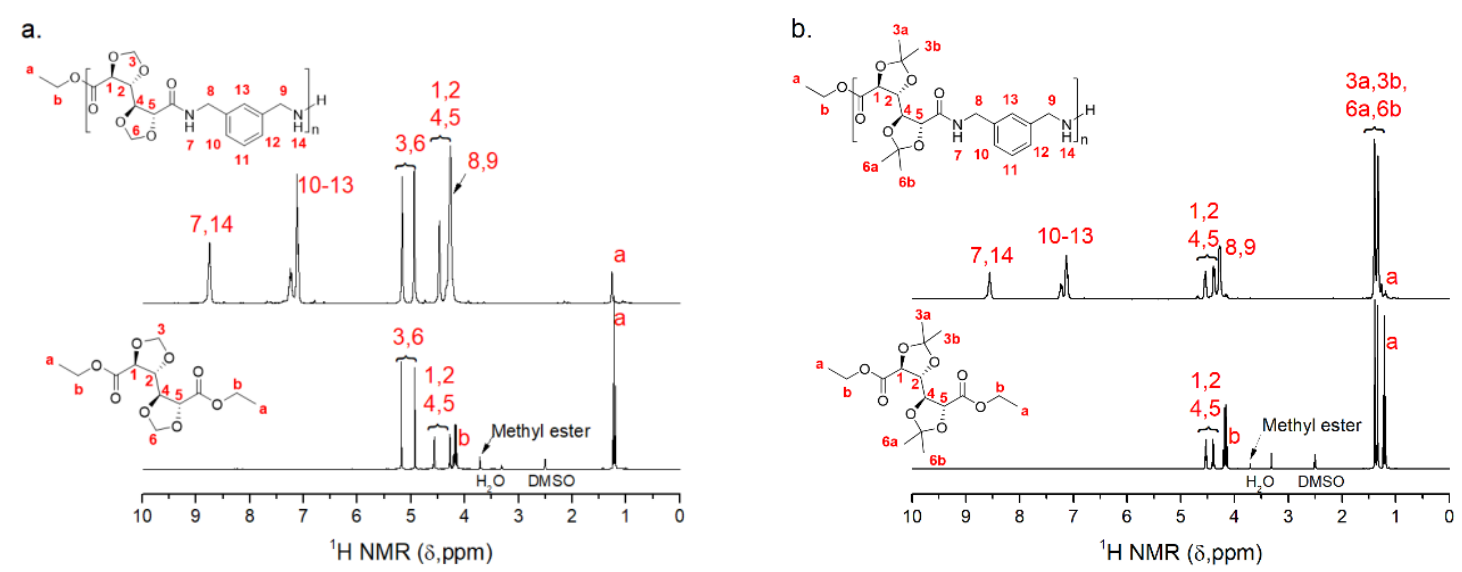

Figure 2-3 ${ }^{l} H$ NMR spectra of a. GalXH diethyl ester and polymer therefrom (PA3) and $b$. GalXMe diethyl ester and polymer therefrom (PA10).

For nearly all polyamides close to expected chemical compositions were obtained. However, for PA4 the biggest deviation from equimolarity in the final polymer was observed as well as a relatively low GalX conversion, explaining the low molecular weight of PA4. The example of the ${ }^{1} \mathrm{H}$ NMR spectrum of galactarates containing polymers is presented in Figure 2-3. Upon polymerization of galactarate the ester protons $b$ are diminishing and new amide protons 7 , 14 are appearing above $8 \mathrm{ppm}$. The analysis of the spectra confirmed that the acetal group can withstand the temperatures above $220^{\circ} \mathrm{C}$.

The preparation of GalX copolymers with aromatic diamines (PPDA or OXDA) via transesterification in the melt was not successful and resulted in low molecular weight polymer with high dispersity (PA6, PA15 in Table 2-1). Moreover, the polymers after melt polycondensation were black and degraded. Since aromatic diamines are weaker nucleophiles due to the delocalization of the amine free electron pair in the aromatic ring, activation methods have to be applied in order to make successful polymers. Previously published literature reports explain that melt polycondensation of aromatic diamines is not a method of first choice since the products of such polycondensations are highly colored, very often crosslinked and contain products resulting from the degradation of aromatic amines used during the reaction. ${ }^{15}$ Besides this, the polymers are characterized by high melting temperatures and glass transition temperatures, which requires application of high temperatures during reaction, very often above the degradation temperature of the polymer/monomer. ${ }^{14}$ Therefore, the 


\section{Chapter 2}

corresponding GalX diacids were activated by phosphorylation and polyamides were obtained by aminolysis of the activated acids in solution at mild temperatures $\left(110{ }^{\circ} \mathrm{C}\right)$ (phosphorylation entries in Table 2-1). ${ }^{16}$ The more bulky OXD resulted in much lower molecular weights than PPDA. It was observed that GalXMe-PPDA polyamides have much higher molecular weight $\left(36 \mathrm{~kg} \cdot \mathrm{mol}^{-1}\right.$ for PA16 in Table 2-1) than GalXH-PPDA polymers $\left(6 \mathrm{~kg} \cdot \mathrm{mol}^{-1}\right.$ for PA7 in Table $\left.2-1\right)$.

Except for the aliphatic reference samples PA1, PA2, PA9 and PA10, all synthesized polymers show a $T_{\mathrm{g}}$ above $100{ }^{\circ} \mathrm{C}$. Among other high $T_{\mathrm{g}}$ polymers, polyamides with MXD (PA11 and PA3 in Table 2-1) had the lowest $T_{\mathrm{g}}$ around $119^{\circ} \mathrm{C}$. This can be explained by the fact that the applied diamine has amine groups attached to two $\mathrm{sp}^{3}$ carbons, which are known to contribute to bigger rotational freedom when incorporated into the polymer backbone. ${ }^{17}$ Comparison between polymers with different aromatic diamines shows how the structural differences influence the glass transition temperatures of polyamides. The highest achieved glass transition temperature was registered for polyamides with $\mathrm{p}$-phenylenediamine, which does not contain any flexible bridges in the structure. From the aromatic diamines, MXD gave polymers with the lowest glass transition region, which can be attributed to abovementioned rotational freedom of the methylene bridges. As expected, the highest $T_{\mathrm{g}}$ values (around $150{ }^{\circ} \mathrm{C}$ or more) were characteristic for rigid, cyclic or bicyclic structures (PA12 - PA14 and PA4 - PA5 in Table 2-1). Semi-aromatic polyamides (PA16, PA17, PA7, PA8) had the highest glass transition temperatures between $180-225^{\circ} \mathrm{C}$.

During the experiments, differences in behavior of the two GalX species were observed. With GalXMe higher conversions could be achieved than with GalXH, still maintaining conventional dispersity values for polycondensation. Rapid reaction of GalXH was observed, resulting in highly viscous polymers which, in most cases, had a higher Đ than the GalXMe equivalents and tended to gel in common organic solvents including 1,1,1,3,3,3hexafluoropropanol. It is hypothesized that the GalXH acetal is more prone to partial deprotection into multiple hydroxyl groups, which can react further with diethyl ester groups and result in branching (below a certain conversion) / crosslinked products (above a certain conversion). However, according to literature ${ }^{18}$ the methylene protection of GalXH should be more chemically resistant than the isopropylene bridge of GalXMe since it is very difficult to remove the protection even in very acidic medium. The branching / crosslinking hypothesis is further supported by the analysis of GPC chromatograms presented in Figure 2-4. The GPC 
curve of the GalXH copolymer after $0.5 \mathrm{~h}$ vacuum is characterized by the appearance of a high molecular weight shoulder indicative for branching, which is absent for the GalXMe copolymer after even $3 \mathrm{~h}$ of vacuum.

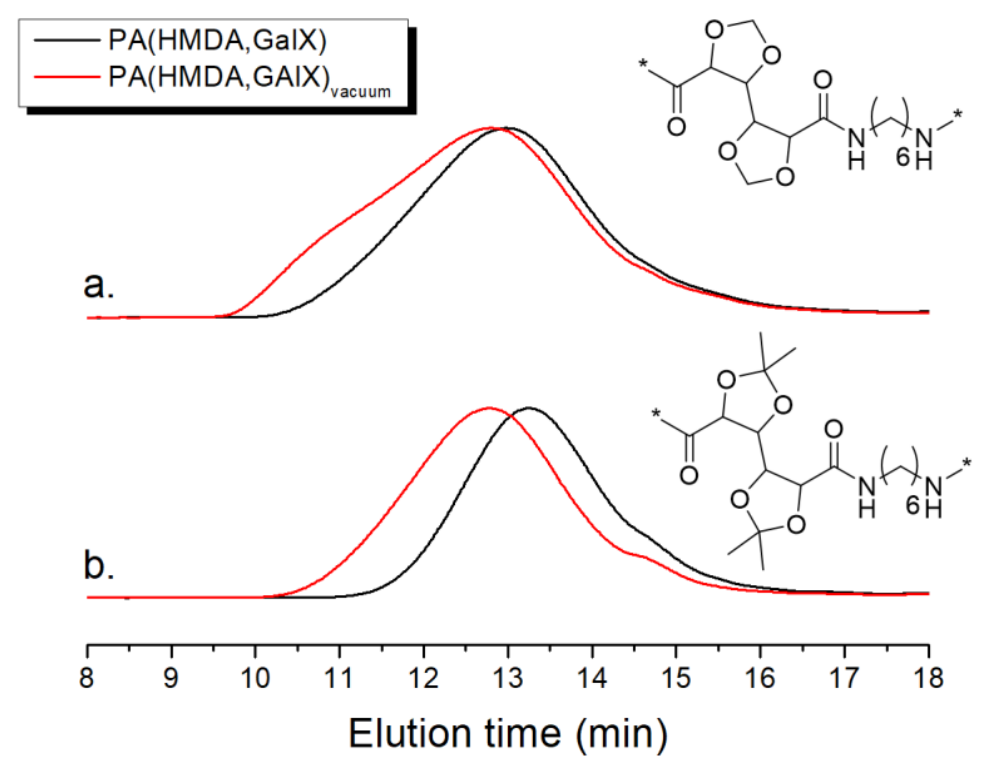

Figure 2-4 GPC chromatogram recorded for (a) poly(HMDA--GalXMe) - PA9 after vacuum corresponds to the sample after 3 h of vacuum, (b) poly(HMDA-GalXH) - PA2 after vacuum corresponds to the sample after 0.5 $h$ of vacuum.

The different behavior of GalXH and GalXMe also explains why copolymerization of GalXH/PACM (entry PA5 in Table 2-1) gave the lowest molecular weight polymer, while even three times higher molecular weights could be achieved with the GalXMe counterpart (entry PA13). The low molecular weight of PA5 can be correlated with the much lower monomer conversions for PA5 (especially the diamine conversion) than for PA13. The conversion for PA5 could not be further improved since the reaction had to be stopped because the polymer viscosity increased a lot and polymer started climbing on the stirrer. This is a sign that gelation is occurring, a phenomenon typically observed with GalXH and not with GalXMe.

The branching / crosslinking hypothesis for GalXH polyamides was further confirmed by the solubility data (Table 2-2). The solubility of the prepared polyamides differed significantly as a function of the used GalX and the character of the diamine (aliphatic or aromatic). Typical GalXH copolymers prepared by melt condensation (PA1 and PA3 in Table 2-1) are only partially soluble in a limited range of solvents (with gelation observed), while GalXMe copolymers (PA9 and PA11 in Table 2-1) show solubility in the majority of the tested 
solvents namely acetone, chloroform, THF, DMSO and HFIP; limited solubility was observed in ethanol. The polymers prepared via the milder reaction conditions using direct polycondensation in solution (PA16 and PA7 in Table 2-1) do not suffer from gelation but confirm a lower solubility of GalXH polymers compared to GalXMe polymers, and additionally show that the incorporation of aromatic diamines lowers the solubility of GalX polyamides in general.

Table 2-2 The solubilities of GalX polyamides obtained in melt polycondensation (PA11,PA3) and direct polycondensation (PA16, PA7).

\begin{tabular}{lllllllll}
\hline Entry & Composition & Water & Ethanol & Acetone & Chloroform & THF & DMSO & HFIP \\
\hline $\boldsymbol{P A 9}$ & GalXMe-HMDA & - & + & + & + & + & + & + \\
$\boldsymbol{P A 1} *$ & GalXH-HMDA & - & - & - & \pm & - & \pm & \pm \\
$\boldsymbol{P A 1 1}$ & GalXMe-MXD & - & + & + & + & + & + & + \\
$\boldsymbol{P A 3}$ & GalXH-MXD & - & - & - & \pm & - & \pm & \pm \\
$\boldsymbol{P A 1 6}$ & GalXMe-PPDA & - & - & - & + & + & + & + \\
$\boldsymbol{P A 7}$ & GalXH-PPDA & - & - & - & - & - & + & + \\
\hline
\end{tabular}

The observed gelation of GalXH is especially apparent after the vacuum stage, when the dispersity of the polymers increases significantly. The difference in behavior between GalXMe and GalXH deserves further investigation. A comparative model study of this problem, including analysis of the degradation products, will be studied in-depth in Chapter 5.

\subsection{Kinetic study}

To better understand the changes that the two types of GalX undergo during polycondensation, conversion (Figure 2-5) and molecular weight (Figure 2-6) development was followed during melt and direct polycondensation (phosphorylation). 
a.

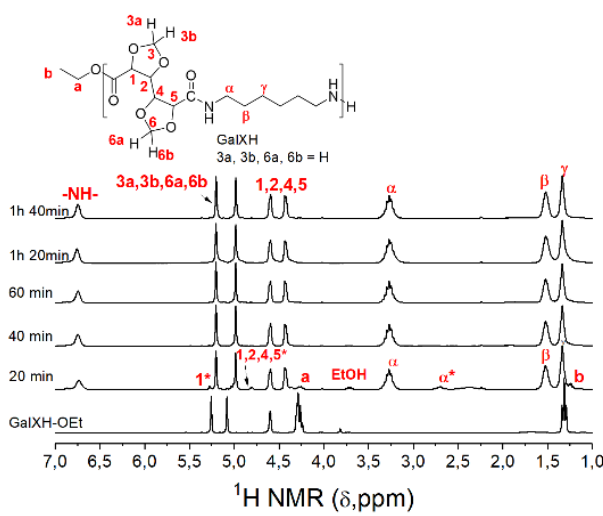

b.

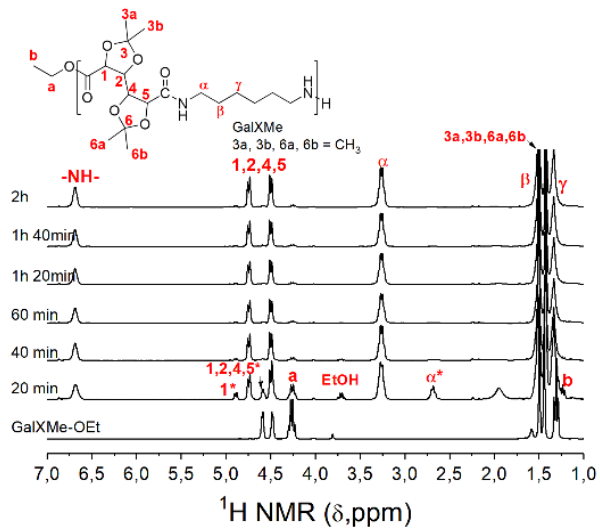

Figure 2-5 ${ }^{1} \mathrm{H} N M R$ spectra $\left(\mathrm{CDCl}_{3}, 300 \mathrm{MHz}\right)$ of (a) GalXH/HMDA amidation reaction in the melt (b) GalXMe/HMDA amidation reaction in the melt. Peaks with * correspond to the unreacted or partially reacted diamine and GalX e.g. $1^{*}$ corresponds to GalX molecule which reacted on one side with diamine, $\alpha^{*}$ corresponds to unreacted diamine.

The analysis of the GalX melt polycondensation kinetics revealed that the two GalX species differ in terms of their reactivity towards diamines. According to ${ }^{1} \mathrm{H}$ NMR (Figure 2-5, Figure 2-6 c) the conversion of GalXMe after the first 20 minutes of the reaction was only $71 \%$ while for GalXH already $88 \%$ conversion had been reached. This difference is a first indication that the more bulky isopropyl protecting group (GalXMe) has influence over the reactivity of the ester carbonyl group. This is in agreement with a previous study about the solution polymerization of diethyl galactarate, diethyl tartarate and 4,5-dimethoxycarbonyl-1,3dioxolane, which revealed that the presence of different heteroatom-containing groups introduced in the $\alpha$ or $\beta$ position can greatly influence the reactivity of the corresponding esters ${ }^{19,20}$. While on the one hand GalXH is faster reacting, forming a polymer earlier, on the other hand, the reaction is difficult to control which causes the increase in the viscosity of the polymer, with a simultaneous increase in the dispersity of the product as shown in Figure 2-6 b. On the corresponding ${ }^{1} \mathrm{H}$ NMR spectra, newly formed GalX backbone $(\mathbf{1 , 2 , 4 , 5 )}$ peaks at $4.72 \mathrm{ppm}$ and $4.52 \mathrm{ppm}$ are visible for GalXMe and at 4.58 and $4.43 \mathrm{ppm}$ for GalXH. For both GalX species peak $1 *$ is observed in the early stage of the reaction and disappears upon continuation of the reaction. This peak was identified as a GalX backbone peak, belonging to a GalX molecule which reacted only at one side with amine (forming amide). This observation is in agreement with the literature. ${ }^{21}$ Diminishing peaks of $\left(\boldsymbol{\alpha}^{*}\right)$ and appearing peaks of amide protons $(\boldsymbol{\alpha})$ indicate that the amine in the very first stage of the reaction is almost fully reacted with GalX.

The evolution of molecular weights for GalXMe-HMDA copolymers and GalXH-HMDA copolymers in Figure 2-6 (a) confirms the faster polymerization and higher molecular weights 
were obtained with GalXH compared to GalXMe. While the dispersity of GalXMe copolymers stays stable during the whole reaction, a rapid increase in dispersity from 2.50 to 4.15 was observed for the GalXH polymers after 60 minutes of reaction time (Figure 2-6 b). These observations, together with the GPC results in Figure 2-4 and the solubility results in Table 2-2, strongly suggest that during melt polycondensation of GalXH side reactions leading to crosslinking occur more often than in the case of GalXMe. It is suspected that due to the partial deprotection of the acetal to free hydroxyl groups during the melt polycondensation the GalXH acetal gives branched/crosslinked products.

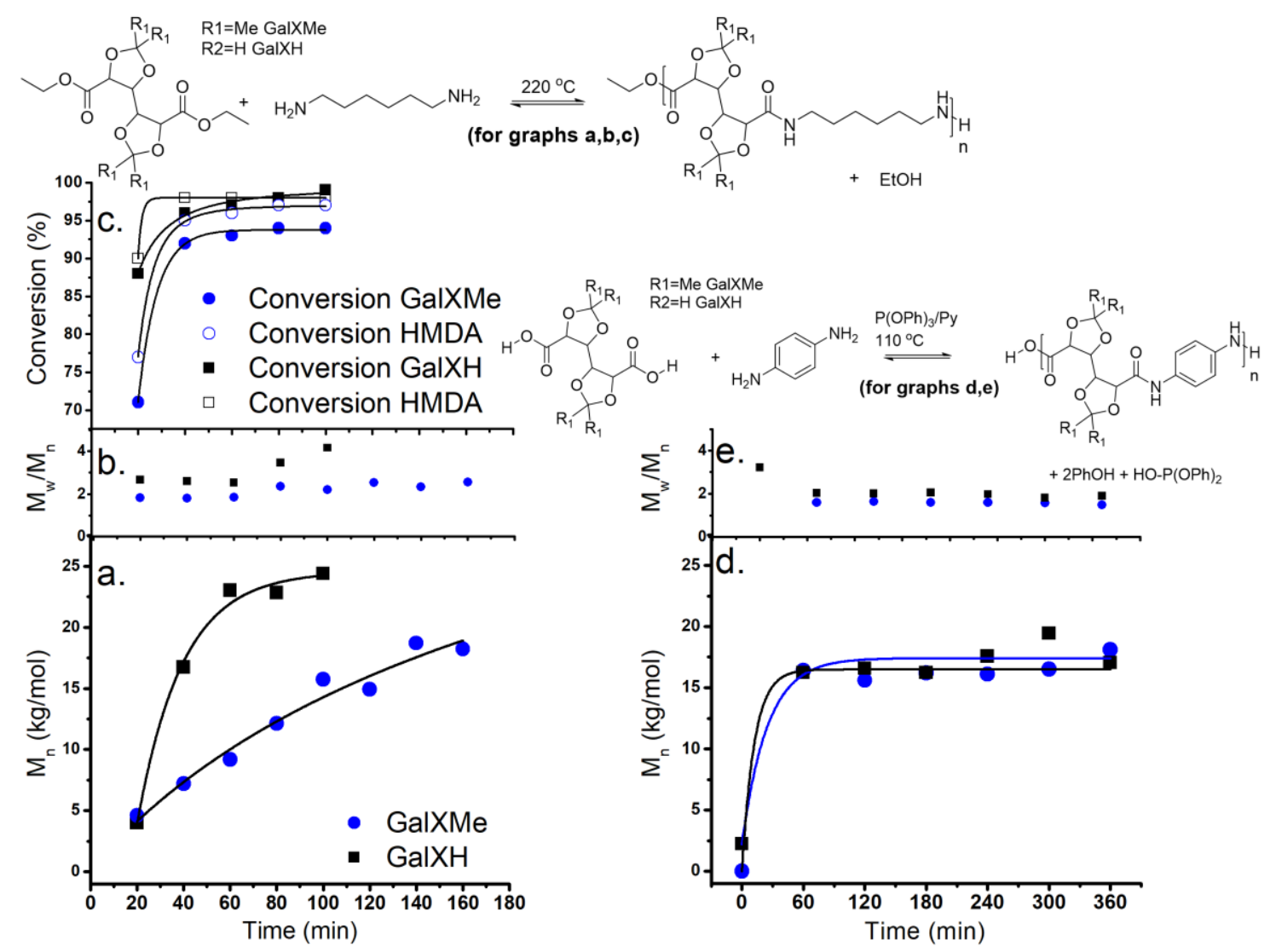

Figure 2-6 Molecular weight, dispersity (GPC) and conversion $\left({ }^{1} H\right.$ NMR) value development during $(a, b, c)$ polycondensation in the melt and $(d, e)$ direct polycondensation via phosphorylation.

The results from the direct polymerization (phosphorylation) in Figure 2-6 (d,e) confirm that $\mathrm{GalXH}$ is more reactive towards amine groups than GalXMe. The first sample $(\mathrm{t}=0)$ in Figure 2-6 (d,e) was taken when the system achieved the reaction temperature of $110{ }^{\circ} \mathrm{C}$ after ramping from room temperature. Already by this time, GalXH had formed a polymer, whereas GalXMe had not. Side reactions during the direct polycondensation in solution (phosphorylation) are greatly reduced, as visible from the fact that dispersities stay stable during the whole reaction. 


\subsection{Mass spectra of polyamides.}

One of each type of polyamide, obtained via direct and melt polycondensation, was investigated using Maldi-Tof. These polyamides have too broad dispersity $Đ$ for this technique to be able to give absolute molecular weights, however the mass spectra confirmed the obtained polymer structures and end groups. On the mass spectra of PA(HMDA,GalXMe) and PA(HMDA,GalXH), obtained via polycondensation in the melt (Figure 2-7), one can observe a couple of different distributions and their corresponding structures are represented in Figure 2-8.

a.

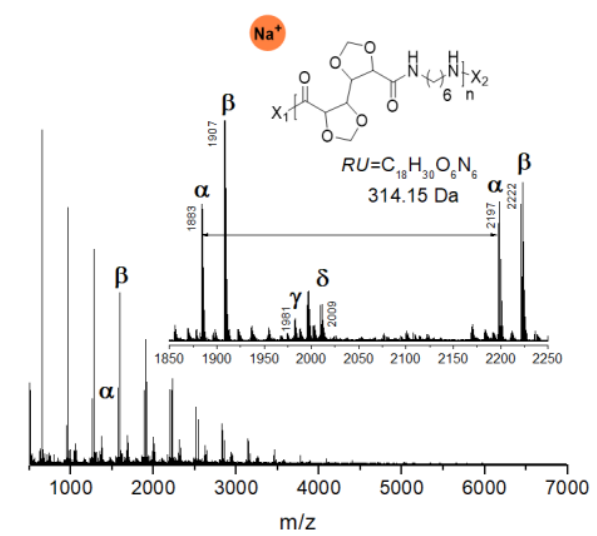

b.

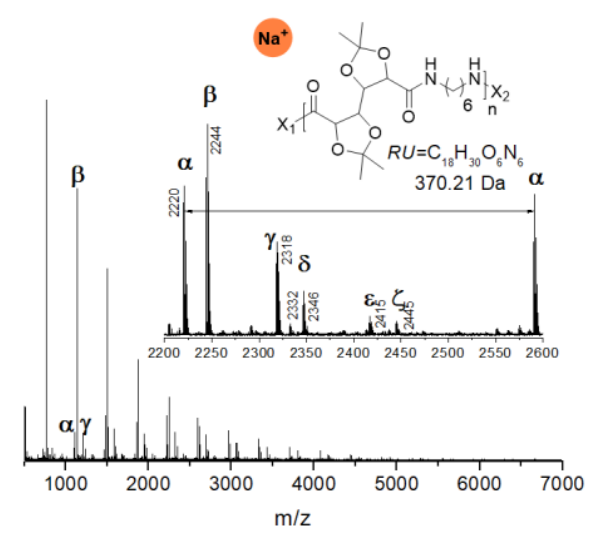

Figure 2-7 Maldi-ToF results for a. PA1 and b. PA9 prepared via melt polycondensation recorded in reflectron mode in dithranol as a matrix and NaTFA as a salt.

The peak with the highest intensity $(\beta)$ corresponds to cyclic structures $n R U+N a^{+}$(see Figure 2-8), whereas peak $\alpha$ can be attributed to the desired linear polyamides with ester end groups at both sides $\mathrm{nRU}+\mathrm{OCH}_{2} \mathrm{CH}_{3}+\mathrm{C}_{12} \mathrm{H}_{16} \mathrm{O}_{6}+\mathrm{OCH}_{2} \mathrm{CH}_{3}+\mathrm{Na}^{+}$. At the lower molecular weight range, peak $\beta$, corresponding to the cyclic structures, is predominant; however, for $\mathrm{m} / \mathrm{z}$ values above 2000-2500 Da, the linear distribution $\alpha$ begins to have higher intensity than distribution $\beta$. A couple of small peaks correspond to fragments of polymer with ethylated (structure $\gamma$ in Figure 2-8) and diethylated (structure $\delta, \varepsilon, \zeta$ in Figure 2-8) amines as end groups and to different side products that can be formed during the hard conditions of melt polycondensation. 


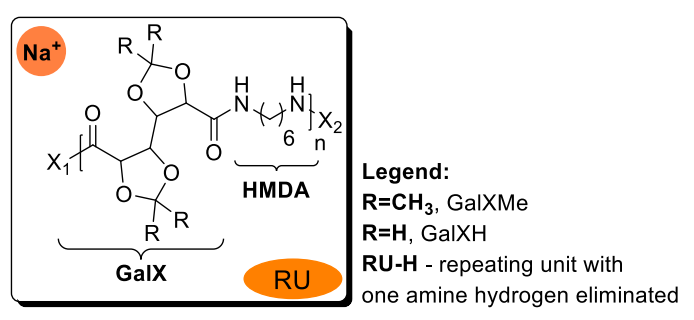

$\alpha: \mathrm{X}_{1}-\mathrm{OCH}_{2} \mathrm{CH}_{3} \mathrm{X}_{2}-\mathrm{GalX}-\mathrm{OCH}_{2} \mathrm{CH}_{3} \quad \delta: \mathrm{X}_{1}-\mathrm{OCH}_{2} \mathrm{CH}_{3} \mathrm{X}-(\mathrm{RU}-\mathrm{H})\left(\mathrm{CH}_{2} \mathrm{CH}_{3}\right)_{2}$

$\varepsilon: \mathrm{X}_{1}-\mathrm{H}-\mathrm{HMDA} \mathrm{X}_{2}-(\mathrm{RU}-\mathrm{H})\left(\mathrm{CH}_{2} \mathrm{CH}_{3}\right)_{2}$
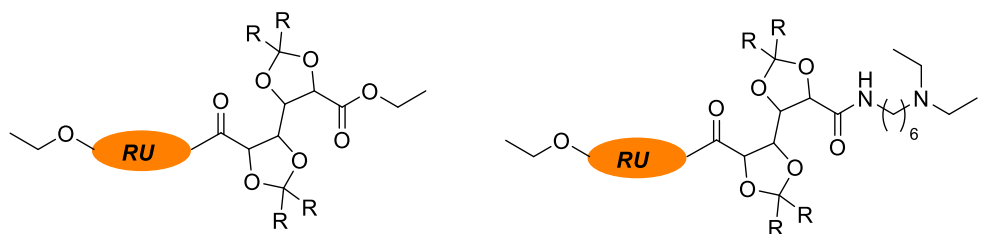

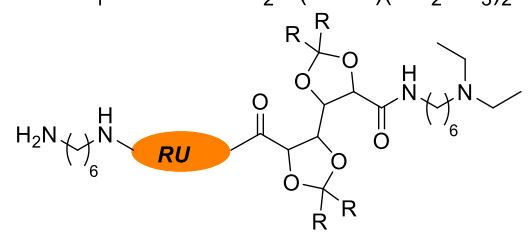

$\beta: \mathrm{X}_{1}-\mathrm{N} / \mathrm{A} \mathrm{X}_{2}-\mathrm{N} / \mathrm{A}$ (cyclic distribution) $\gamma: \mathrm{X}_{1}-\mathrm{OCH}_{2} \mathrm{CH}_{3} \mathrm{X}_{2}-\mathrm{RU}-\mathrm{CH}_{2} \mathrm{CH}_{3}$

$\zeta: \mathrm{X}_{1}-\mathrm{HMDA}-\mathrm{CH}_{2} \mathrm{CH}_{3} \mathrm{X}_{2}-(\mathrm{RU}-\mathrm{H})\left(\mathrm{CH}_{2} \mathrm{CH}_{3}\right)_{2}$

$R U$

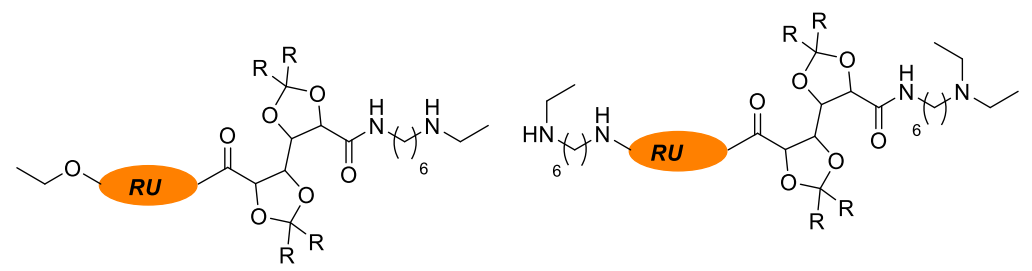

Figure 2-8 Distributions present in the Maldi-ToF spectrum recorded for GalX PAs.

Since the molecular weight of PA16 prepared via direct polycondensation (i.e. phosphorylation with triphenyl phosphite esters) is rather high, a polymer with lower molecular weight was made in order to be able to see a representative fraction of the polymer in reflectron mode. Due to multiple filtrations, two different fractions were isolated with $M_{n}=4800 \mathrm{~g} \cdot \mathrm{mol}^{-1}, \mathrm{Ð}=1.29$ and $M_{n}=1800 \mathrm{~g} \cdot \mathrm{mol}^{-1}, \mathrm{Ð}=1.75$. Both spectra are overlaid in Figure 2-9.
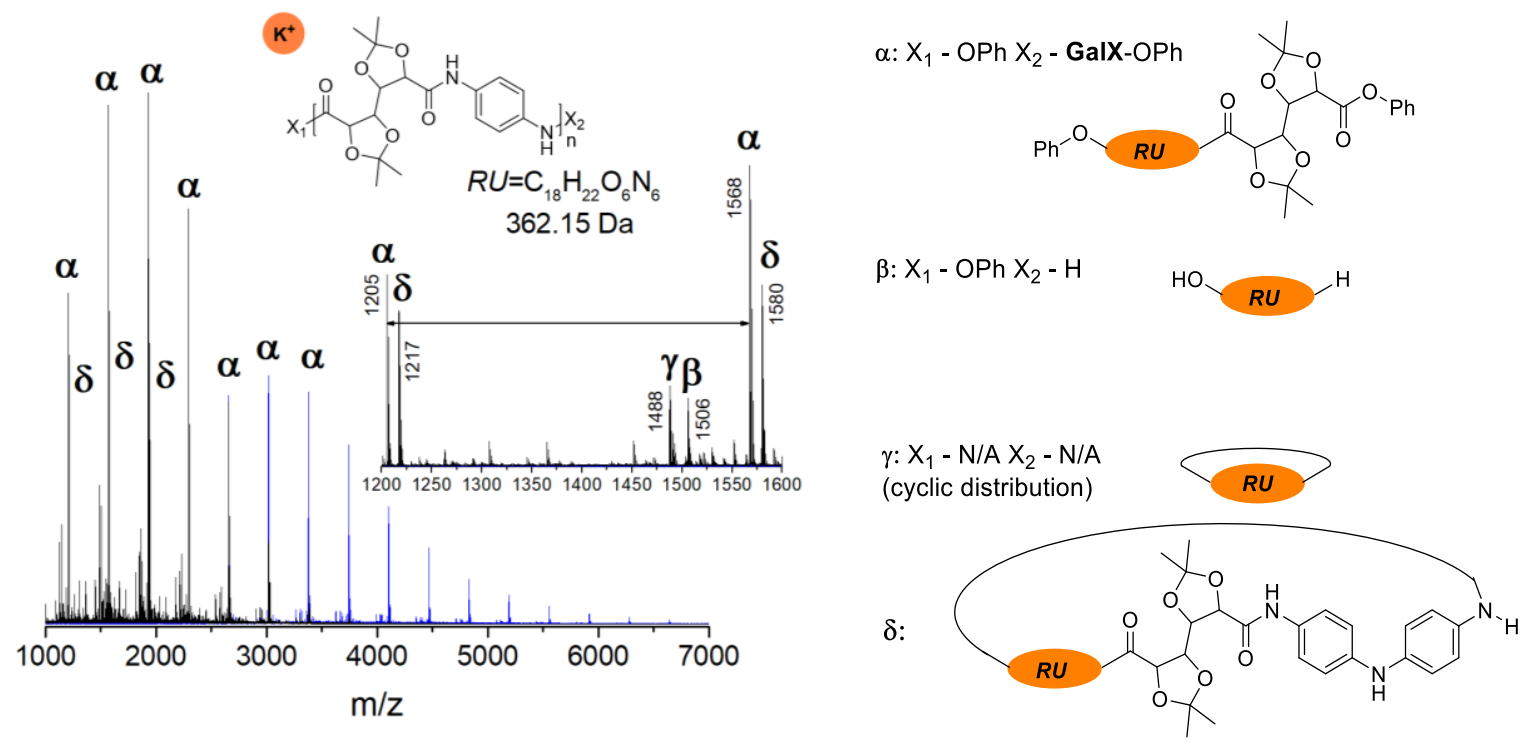

Figure 2-9 Maldi-Tof results for PA(PPDA,GalXMe) prepared via direct polycondensation for low molecular weight polymer (GPC: $M_{n}=4800 \mathrm{~g} \cdot \mathrm{mol}^{-1}, \emptyset=1.29$ (blue distribution) and $G P C: M_{n}=1800 \mathrm{~g} \cdot \mathrm{mol}^{-1}, \emptyset=1.75$ (black distribution) recorded in reflectron mode in dithranol as a matrix and KTFA as a salt. 
First of all, analysis of the obtained fragmentation patterns showed that in both fractions linear, GalX terminated chains are predominant $\left(\alpha-\mathrm{nRU}+\mathrm{OPh}+\mathrm{C}_{12} \mathrm{H}_{16} \mathrm{O}_{6}+\mathrm{OPh}+\mathrm{K}^{+} \mathrm{m} / \mathrm{z}=1205\right.$, $1568, \ldots \mathrm{Da}$ ) which is induced by the use of an excess of acid-functional monomer during the preparation of low molecular weight polyamide. Due to the decomposition of triphenyl phosphite during the phosphorylation reaction, phenol is formed as a by-product which competes in the reaction environment with the aminolysis of the activated ester by the diamine. Due to this phenomenon the final product has phenol ester end groups, rather than the free carboxylic end groups. It was suspected that the phenyl ester can further undergo hydrolysis by methanol which is used to precipitate the polymer, but a peak corresponding to methyl ester end groups was not identified.

The presence of a polymer, which was end-capped with the free carboxylic function of GalX was also observed, but on both spectra the corresponding distribution $\beta$ was of very low intensity. Additionally, further analysis of the spectra revealed the presence of cyclic structures $\left(\gamma-\mathrm{nRU}+\mathrm{K}^{+}\right)$with increasing intensity in the lower molecular weight region.

Besides those obvious distributions, additional distribution $\delta$ was identified which differed from the cyclic distribution by one phenyl ring with an additional amine group (Figure 2-9, distribution $\delta$ ). This odd combination is presumably a result of the self-condensation of the diamine.

\subsection{Hydrolytic stability}

Since the presented polyamides are addressed for a variety of applications in different environments, their hydrolytic stability is of interest. Indeed it is even more significant with these polyamides since the protecting groups from GalX are potentially susceptible to hydrolysis, especially in the presence of acids. ${ }^{18}$ The hydrolysis experiment was performed in two different acidic media: 90\% formic acid and 5\% citric acid solution as a compound present in food and drinks. The experiment was conducted over 2 hours at room temperature in the aggressive medium of formic acid and over 60 days in a solution of citric acid, whereafter samples were analyzed using GPC and NMR.

The influence of the concentrated acid has a different impact on the two GalX derivatives. After two hours in such an aggressive medium the structure of GalXH-HMDA seems not to be influenced by the strongly acidic conditions (Figure 2-10). After exposition to the same conditions, for the GalXMe polymer PA9 hydroxyl groups are observed between $4-6$ ppm 


\section{Chapter 2}

coming from the deprotection of the ketal. This phenomenon confirms that GalXH polymers are more chemically resistant and appropriate for use in harsher conditions. Moreover, those observations were confirmed by similar results obtained during investigation of hydrolytic stability of GalXH copolyesters ${ }^{9}$ and deprotection experiments of GalXMe polyamides with trifluoroacetic acid. ${ }^{12,22}$ The scission of amide linkage was not observed for both polyamides (Figure 2-10 a and b).

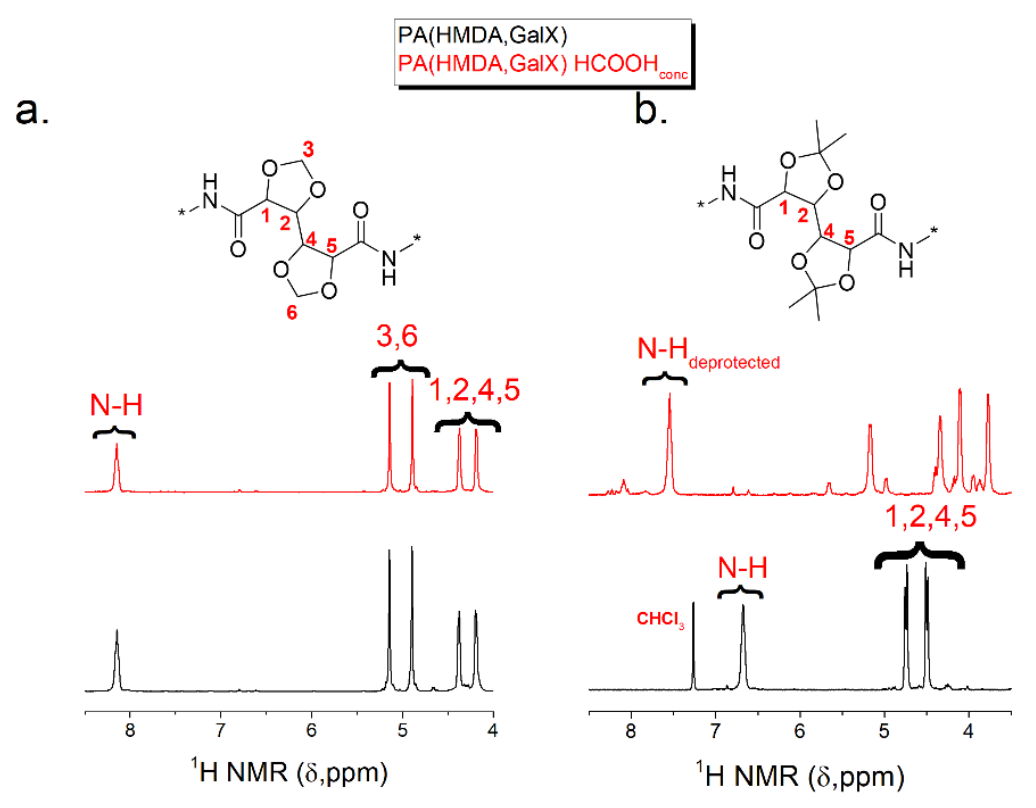

Figure 2-10 ${ }^{1} \mathrm{H}$ NMR spectrum recorded in DMSO-d or $_{6} \mathrm{CDCl}_{3}$ for) a. PAI (HMDA-GalXH) and b. PA9 (HMDA-GalXMe) before and after 2 h 90\% Formic Acid.

In contrast to the influence of the strongly acidic medium, a mild condition of $5 \%$ citric acid seems not to influence the polymer even after prolonged exposition over 60 days. The GPC chromatogram in Figure 2-11 reveals that the applied mildly acidic conditions do not influence the molecular weight of the polyamides. Similar results are obtained when analyzing proton NMR spectra before and after hydrolysis. Those results show that polymer containing acetal moieties, whether it is the more stable O-methylene protection or the less stable O-isopropylidene protection, can withstand acidic conditions and therefore be used as materials in many applications like containers for refreshments or boilers. 
a.
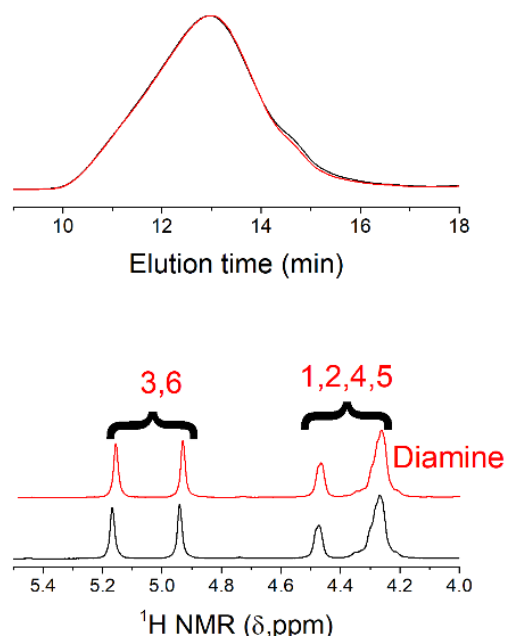

b.
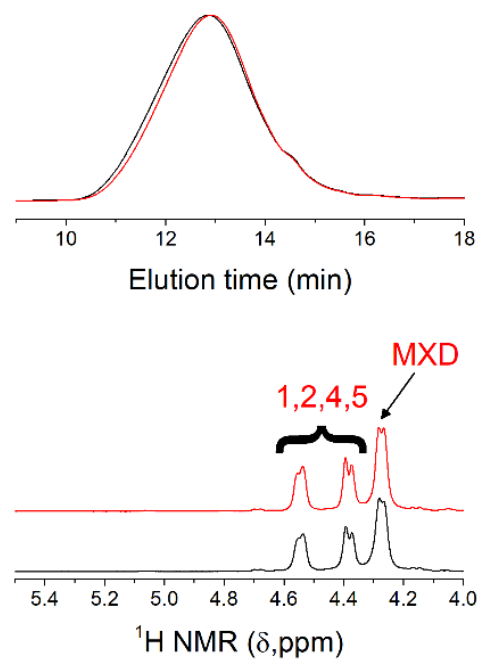

Figure 2-11 ${ }^{1} \mathrm{H}$ NMR spectrum recorded in DMSO-d ${ }_{6}$ or $\mathrm{CDCl}_{3}$ and GPC chromatograms (HFIP) for a. PA3 (MXD-GalXH) and b. PA11 (MXD-GalXMe) before and after 60 days in 5 wt\% solution of citric acid with $p H=1.9$.

\subsection{Thermal stability}

A significant negative aspect of bio-based molecules is their thermal stability, which in many cases is a limiting factor for elevated temperature applications and for processing. Thermogravimetric analysis of the polymers shows that the obtained polymers are stable at the reaction temperature (i.e. $220{ }^{\circ} \mathrm{C}$ ) and that it is possible to process them below their degradation temperature (Table 2-3).

During polycondensation, a vast number of amide bonds are already formed at temperatures lower than $100{ }^{\circ} \mathrm{C}$. The amide-containing oligomers are much more stable at synthesis temperature $\left(220^{\circ} \mathrm{C}\right)$ than the monomer, and as such successful polymerization at high temperatures is possible. All synthesized polymers show degradation temperatures above $300{ }^{\circ} \mathrm{C}$ for $5 \%$ weight loss.

Moreover, isothermal experiments were prepared to investigate the stability of the polymers exposed to elevated temperatures for a longer period of time. Polyamide PA11 does not seem to be affected by the temperature of $210^{\circ} \mathrm{C}$. After 8 hours of isothermal program, the sample weight had only decreased by $2 \%$, which is a rather negligible value and is too low to be interpreted as degradation; instead perhaps caused by the reaction of end groups or simply the loss of residual water, which was not removed during drying. When the isothermal 
stability of the polymer is followed at $280{ }^{\circ} \mathrm{C}, 76 \%$ of the polymer weight was left in the TGA pan. This indicates slow degradation, and suggests that the polymer can withstand short exposure to high temperatures (important for processing presented in Chapter 7).

Table 2-3 Thermogravimetric analysis of selected polymers prepared from GalX.

\begin{tabular}{|c|c|c|c|c|}
\hline Entry & Composition & $\begin{array}{l}T_{5 \%} \\
\left({ }^{\circ} \mathrm{C}\right)\end{array}$ & $\begin{array}{l}T_{10 \%} \% \\
\left({ }^{\circ} \mathrm{C}\right)\end{array}$ & $\begin{array}{c}T_{\max } \\
\left({ }^{\circ} \mathrm{C}\right)\end{array}$ \\
\hline \multicolumn{5}{|c|}{ Heating at $10^{\circ} \mathrm{C} / \mathrm{min}$} \\
\hline$P A 1$ & GalXH-HMDA & 332 & 361 & 403 \\
\hline PA9 & GalXMe-HMDA & 342 & 367 & 399 \\
\hline PA16 & GalXMe-PPDA & 316 & 333 & 351 \\
\hline PA17 & GalXMe-OXDA & 304 & 330 & 349 \\
\hline \multicolumn{5}{|c|}{ Isothermal } \\
\hline Entry & Composition & $\begin{array}{l}T_{\text {iso }} \\
\left({ }^{\circ} \mathrm{C}\right)\end{array}$ & $\begin{array}{l}t_{\text {iso }} \\
\text { (h) }\end{array}$ & $\begin{array}{l}m_{\text {iso }} \\
(\%)\end{array}$ \\
\hline$P A 11$ & GalXMe-MXD & 210 & 10 & 98 \\
\hline$P A 11$ & GalXMe-MXD & 280 & 10 & 76 \\
\hline
\end{tabular}

$\mathrm{T}_{5} \% \mathrm{~T}_{10 \%}$ - temperature corresponding to $5 \%$ and $10 \%$ weight loss respectively, $\mathrm{T}_{\max }-$ temperature corresponding to maximum rate of weight loss $\left({ }^{\circ} \mathrm{C}\right) /$ first derivative, $\mathrm{T}_{\text {iso }}$ - temperature of the isothermal program, tiso - duration of the isothermal program, miso - weight of sample after isothermal experiment

\subsection{Rheological properties of GalX polymers}

The viscosity of amorphous polymers depends on the structure of polymer, weight-average molecular weight and on the temperature. The effect of the temperature on the polymer melt (flow) is widely acknowledged as a complex issue. ${ }^{23}$ Typically, a complex viscosity $\left(\eta^{*}\right)$ is measured on a rheometer and can be expressed as a function of the loss modulus G" or storage modulus $G^{\prime}$ and the angular frequency, but it can also be directly plotted as a function of temperature etc. Amorphous polymers usually loose viscosity above the glass transition temperature, in which they reach the rubbery plateau and subsequently start to flow. The presence and the length of the rubbery plateau strongly depend on the interactions between polymeric chains. 


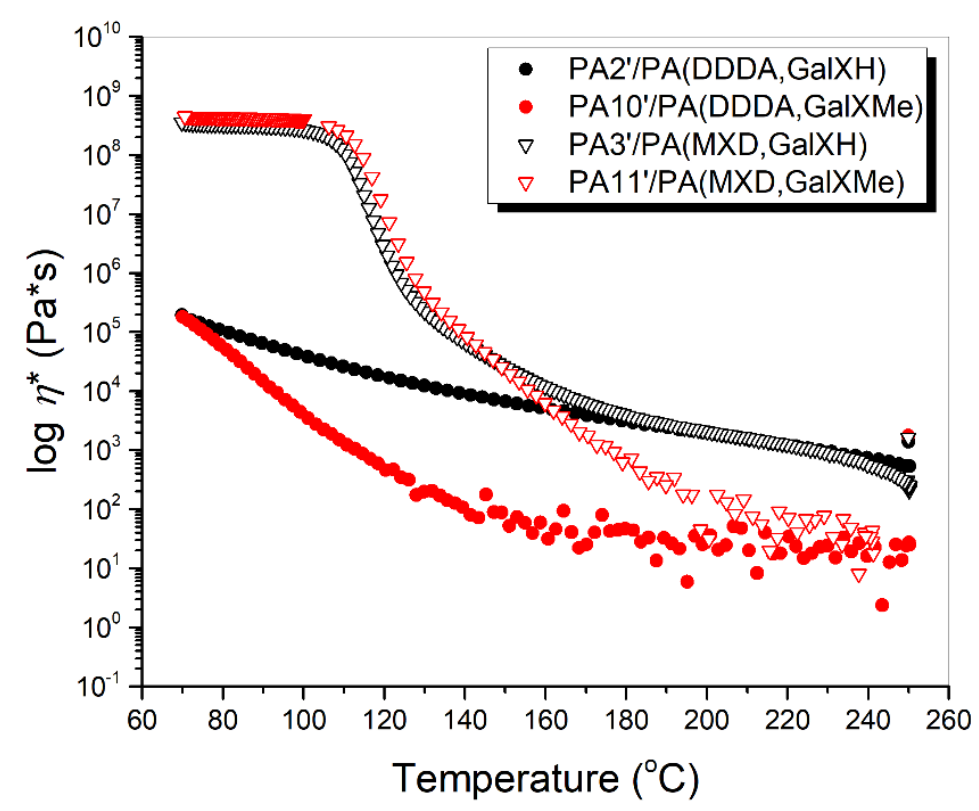

Figure 2-12 The viscoelastic behavior of GalX polyamides represented by the complex viscosity (log $\left.\eta^{*}\right)$ as a function of temperature for PA2' $\left(\mathrm{Tg}=49^{\circ} \mathrm{C}, \mathrm{Mw}=61.1 \mathrm{~kg} \cdot \mathrm{mol}-1, \emptyset=4.7\right), P A 10^{\prime}\left(\mathrm{Tg}=51^{\circ} \mathrm{C}, \mathrm{Mw}=56.7 \mathrm{~kg} \cdot \mathrm{mol}\right.$ 1, $Đ=2.7), P A 3^{\prime}\left(T g=125^{\circ} \mathrm{C}, \mathrm{Mw}=65.8 \mathrm{~kg} \cdot \mathrm{mol}-1, \mathrm{~T}=4.7\right)$ and $P A 11^{\prime}\left(\mathrm{Tg}=119^{\circ} \mathrm{C}, \mathrm{Mw}=32.3 \mathrm{~kg} \cdot \mathrm{mol}-1, \mathrm{D}=1.7\right)$ recorded for the constant angular frequency $\omega=1 \mathrm{rad} / \mathrm{s}$.

The polymer sample was loaded on the rheometer at $250^{\circ} \mathrm{C}$ and subsequently the temperature was decreased to $60^{\circ} \mathrm{C}$ at a cooling rate of $5^{\circ} \mathrm{C} / \mathrm{min}$. Polymers referred to as PA2', PA3', PA10' PA11' and PA12' in this subchapter are structurally identical to PA2, PA3, PA10, PA11 and PA12 characterized in Table 2-1, but have different molecular weights since they belong to a different batch (see experimental part Table 2-5). The viscosity of GalX polyamides in the temperature range $60-250{ }^{\circ} \mathrm{C}$ represents a typical plot obtained for amorphous polymers (Figure 2-12). The viscosity of the presented polymers is decreasing with temperature, however, the curve analysis indicates that GalXMe polyamides, regardless the structural differences between used diamines, possess lower viscosities above $200{ }^{\circ} \mathrm{C}$ than the analoguous GalXH polymers. The presented data shows that polymers prepared from GalXH possess a rubbery plateau whereas GalXMe viscosity rapidly decreases with the increase of the temperature and polymer quickly reaches the viscous region. This could mean that GalXH chains possess strong interchain interactions (form sufficient entanglements thus rubbery plateau is more pronounced) and for GalXMe the intrachain interactions are dominating over the interchain ones (thus less entanglements).

The complex interactions of the dioxolane moieties incorporated into polyamides will be elucidated in chapter 6 . 

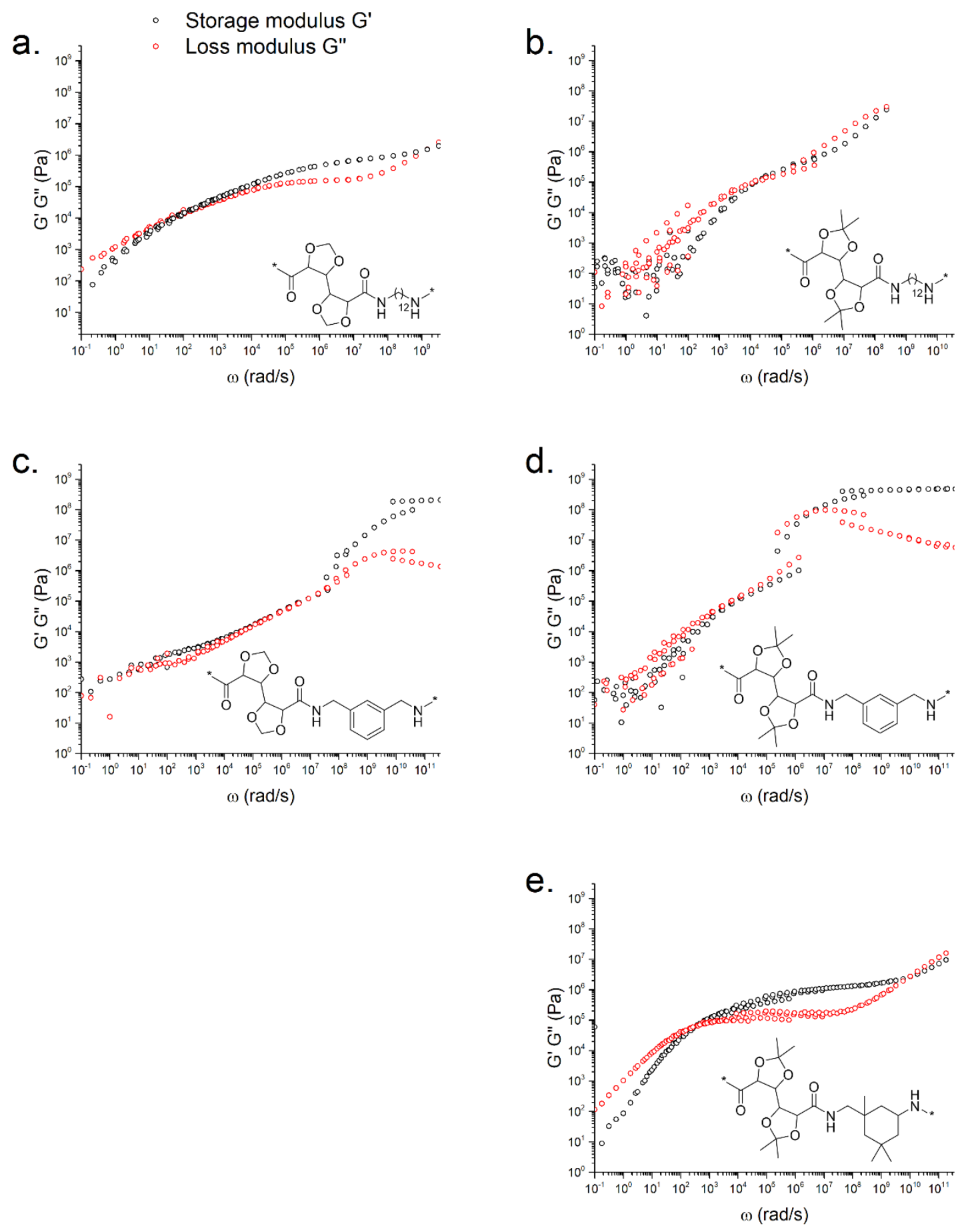

Figure 2-13 The rheological profiles of storage modulus $\left(G^{\prime}\right)$ and loss modulus ( $G$ ') as a function of angular frequency a. PA2' $\left(T_{g}=49{ }^{\circ} \mathrm{C}, M_{w}=61.1 \mathrm{~kg} \cdot \mathrm{mol}^{-1}, D=4.7\right), b . P A 10^{\prime}\left(T_{g}=51{ }^{\circ} \mathrm{C}, M_{w}=56.7 \mathrm{~kg} \cdot \mathrm{mol}^{-1}, D=2.7\right), \mathrm{c}$. PA3' $\left(T_{g}=125^{\circ} \mathrm{C}, M_{w}=65.8 \mathrm{~kg} \cdot \mathrm{mol}^{-1}, D=4.7\right), \mathrm{d} . \mathrm{PAll}^{\prime}\left(T_{g}=119^{\circ}{ }^{\circ} \mathrm{C}, M_{w}=32.3 \mathrm{~kg} \cdot \mathrm{mol}^{-1}, \mathrm{D}=1.7\right)$. and e. PA12' $\left(T_{g}=155^{\circ} \mathrm{C}, M_{w}=28.9 \mathrm{~kg} \cdot \mathrm{mol}^{-1}, Ð=1.7\right)$.

Typically, the frequency sweep profiles of linear, amorphous polymers show different regions. A viscous region present at lower angular frequencies, which typically has a storage modulus (G') lower than the loss modulus (G'). A rubbery plateau starts after the cross-over of the G' and $G$ ' plots with increasing angular frequencies. After those two regions, there is also a 
transition region and a glassy region. The location of those transitions depends on relaxation times of polymeric fragments and can be directly related to the weight-average molecular weight of the polymer as well as the molecular weight between the entanglements. The frequency sweep measurement performed for different GalXH and GalXMe polyamides revealed that polyamides containing one of the two GalX in combination with aliphatic 1,12dodecanediamine (Figure 2-13(a) and (b)) or cycloaliphatic IPDA (Figure 2-13(e)) have profiles which are typical for amorphous, linear polyamides. The polyamide of DDDA and GalXMe has a narrow rubbery plateau in comparison to GalXH, which usually suggest that $\mathrm{M}_{\mathrm{w}}$ is low compared to the entanglement molecular weight. Since the $\mathrm{M}_{\mathrm{w}}$ of both polyamides is similar (see Table 2-5), it strongly suggests that the interchain interactions in GalXMe are less pronounced than in GalXH polymers. PA(MXD,GalXH) in Figure 2-13(c) shows only one cross-over point, has a value of the storage modulus $G$ ', lower than that of loss modulus $\mathrm{G}^{\prime}$ at lower frequencies and additionally in a certain range the two plots ( $\mathrm{G}^{\prime}$ and $\left.\mathrm{G}^{\prime}\right)$ are parallel to each other. The PA(MXD,GalXMe) in Figure 2-13(d) shows a similar trend, with the exception that at lower frequencies the loss modulus $\mathrm{G}^{\prime}$ is lower than the storage modulus G”. Typically, those types of variations might indicate the formation of some kind of covalent or non-covalent network. In case of PA(MXD,GalXH) the storage modulus (G') dominates over the loss modulus (G') and in case of $\mathrm{PA}(\mathrm{MXD}, \mathrm{GalXMe})$ the relation is opposite, which suggests that the density of the network of $\mathrm{PA}(\mathrm{MXD}, \mathrm{GalXH})$ is higher than of PA(MXD,GalXMe). Both profiles (c) and (d) in Figure 2-13 show some irregularities, which might originate from (a) a cross-linking occurring during the measurement as well as from the presence of a (b) covalent or (c) non-covalent polymer network already prior to measurement (e.g. $\pi-\pi$ ). It was already shown that GalXH tends to cross-link at higher temperatures. In order to investigate this phenomenon the GPC traces were recorded before and after the measurement. The molecular weight distribution of the polymer before rheology compared with the distribution after the measurement reveals that PA3' (PA(MXD,GalXH)) after rheology is barely soluble in HFIP, forming a gel rather than dissolving (Figure 2-14 (a)) which indicates cross-linking during the measurement (option (a)). PA11' (PA(MXD,GalXMe)), on the other hand, does not undergo covalent cross-linking during the rheology measurement, however it does not seem like the polymer is fully linear. One of the possible explanations is the occurrence of non-covalent $\pi-\pi$ stacking or amide group aromatic ring interactions or branching, which occurred during the polymer preparation. The previously described domination of storage modulus over the loss modulus for 
PA(MXD,GalXH) might constitute additional confirmation that the interactions in the GalXH polyamide network are generally stronger or that the network is denser than interactions in the GalXMe polymer network. The results obtained via rheology are in agreement with the previous observation (e.g. obtained dispersities during the synthesis of polyamides and gelation of polyamides in organic solvents) that GalXH polyamides have the tendency to cross-link at higher temperatures, forming a covalent network (alternatively branched polymer). The presence of shear is expected to amplify this phenomenon.

In chapter 7 two of the presented polymers with elevated glass transition temperatures will be processed, namely PA(MXD,GalXMe) and PA(IPDA,GalXMe). GalXH polyamides were not chosen for further processing experiments due to the possibility of cross-linking during processing. The profile of PA(IPDA,GalXMe) represents the typical behavior of amorphous, linear polyamide indicating that the polyamide should be stable during the experiment in the extruder.

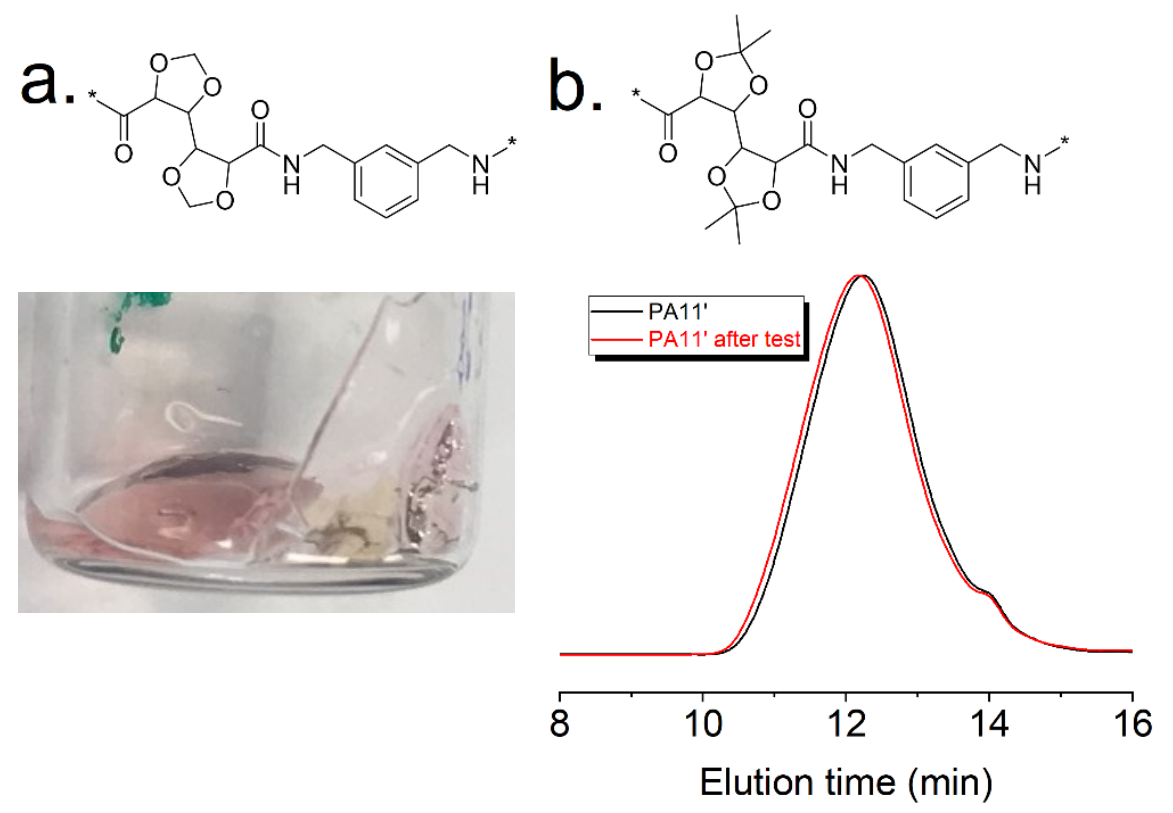

Figure 2-14 The changes recorded after the rheological measurement for a. PA3'image of a gel obtained upon immersion of the postrheology polymer in HFIP and b. PA11': GPC traces before and after the rheology measurement.

\subsection{FT-IR analysis of homopolyamides}

Typical FT-IR spectra of homopolyamides with and without GalX are shown in Table 2-4. Typically, the region above $2800 \mathrm{~cm}^{-1}$ is used for identification of amide groups $\left(\sim 3300 \mathrm{~cm}^{-}\right.$

${ }^{1}$ corresponding to the stretching $(v)$ vibration of $\left.\mathrm{N}-\mathrm{H}\right)$., The spectra for time-resolved or 
temperature-resolved analysis are normalized via the peaks for the stretching vibrations of the C-H of methylene or methyl groups (2990-2850 $\left.\mathrm{cm}^{-1}\right)$ spectra. Signals present at 1634 and $1532 \mathrm{~cm}^{-1}$ are also related to the stretching vibration typical for amides, namely Amide I and Amide II vibrational modes, respectively.

Two signals at 1470 and $1420 \mathrm{~cm}^{-1}$ correspond to deformations $(\delta)$ of methylene C-H's attached to amides and to the carbonyl side of the amide bond, respectively. This region is used in conformational analysis for the identification of the gauche and trans orientation of amide bonds in polyamides. ${ }^{24,25} \mathrm{~A}$ band at $1420 \mathrm{~cm}^{-1}$ is not present in both GalX polyamide spectra (PA2 and PA10) due to the lack of methylene groups connected to the carbonyl side of the amide bond. The band at $1470 \mathrm{~cm}^{-1}$ originates from $\mathrm{C}-\mathrm{H}$ vibrations of methylene units connected to the nitrogen from the amide group and is characteristic for chains which adopt trans conformation. ${ }^{26}$ The trans conformation is substantially disrupted for GalX-based polyamides probably due to limited rotational freedom of the methylene units resulting from rigidity of GalX fragments. Peaks between $1460-1440 \mathrm{~cm}^{-1}$ originate from the vibrations of methylene units attached to the $\mathrm{NH}$ side of the amide bond which adopt gauche conformation. ${ }^{27}$ 
Table 2-4 FT-IR spectra of polyamides PA(DDDA,GalXH) (PA2), PA(DDDA,GalXMe) (PA10) and aliphatic diacid and diamine $(P A(D D D A, 10))$ with signal assignment.

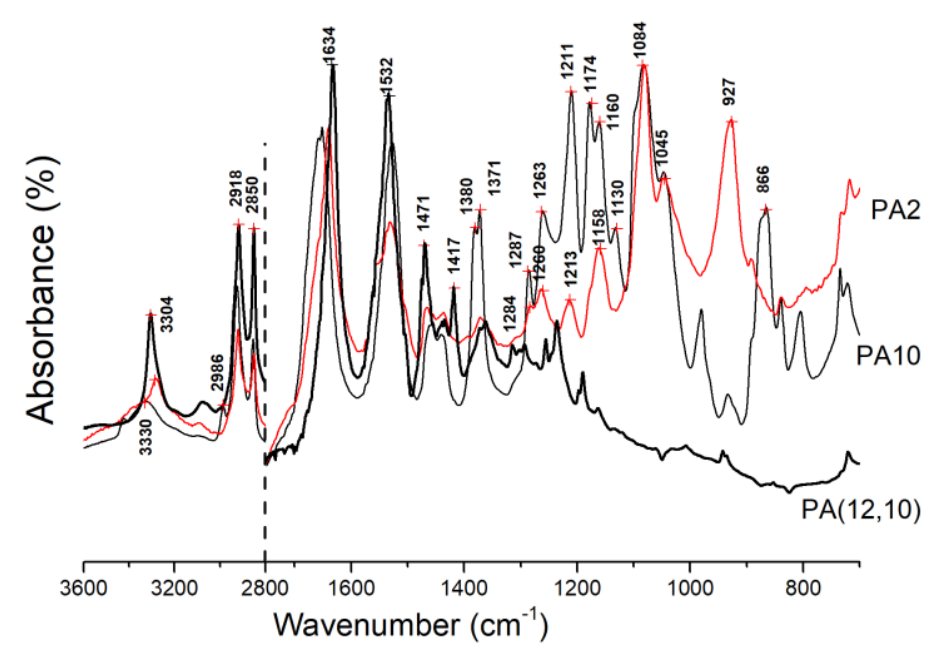

\begin{tabular}{|c|c|c|}
\hline Wavenumber $\left(\mathrm{cm}^{-1}\right)$ & Vibration & Comment \\
\hline$>3200$ & $v(\mathrm{~N}-\mathrm{H})$ & - \\
\hline 2986 & $v(\mathrm{C}-\mathrm{H}) \mathrm{CH}_{3}$ & - \\
\hline 2920,2850 & $v(\mathrm{C}-\mathrm{H}) \mathrm{CH}_{2}$ & - \\
\hline 1634 & Amide $\mathrm{I}, \mathrm{v}(\mathrm{C}=\mathrm{O})$ & - \\
\hline 1532 & Amide II, $\delta(\mathrm{N}-\mathrm{H})$ & - \\
\hline$\sim 1470$ & $\delta(\mathrm{C}-\mathrm{H})\left(\mathrm{CH}_{2}-\mathrm{NH}\right)_{\text {trans }}$ & $\begin{array}{l}\text { Typical deformation modes for } \\
\text { aliphatic nolvamides }\end{array}$ \\
\hline$\sim 1460-1440$ & $\delta(\mathrm{C}-\mathrm{H})\left(\mathrm{CH}_{2}-\mathrm{NH}\right)_{\text {gauche }}$ & \\
\hline$\sim 1420$ & $\delta(\mathrm{C}-\mathrm{H})\left(\mathrm{CH}_{2}-\mathrm{C}=\mathrm{O}\right)$ & \\
\hline $1370-1380$ & $\delta(\mathrm{C}-\mathrm{H})\left(\mathrm{CH}_{3}\right)$ & $\begin{array}{l}\text { Assymetric doublet typical for } \\
\text { isopropylidene fragment }\end{array}$ \\
\hline $1290-1260$ & Amide III, $v(\mathrm{C}-\mathrm{N}), \delta(\mathrm{N}-\mathrm{H})_{\text {in-plane }}$ & - \\
\hline $\begin{array}{c}1213,1158(\mathrm{GalXH}) \\
1211,1174,1160,1130 \\
\text { (GalXMe) } \\
1084,1045 \text { (GalXH and } \\
\text { GalXMe) }\end{array}$ & $v(\mathrm{C}-\mathrm{O}-\mathrm{C})$ & $\begin{array}{l}\text { Typical region for C-O vibrational } \\
\text { modes }\end{array}$ \\
\hline $\begin{array}{l}927(\text { GalXH) } \\
866(\text { GalXMe })\end{array}$ & $v(\mathrm{C}-\mathrm{O}-\mathrm{C})$ coupled with C-C & $\begin{array}{l}\text { Very characteristic region which } \\
\text { distinguishes GalXH and GalXMe ring }\end{array}$ \\
\hline
\end{tabular}

There are two regions, which can be used to distinguish between the two different types of dioxolane fragments. First, a typical assymetric doublet at $1380-1370 \mathrm{~cm}^{-1}$ corresponds to the $\mathrm{C}-\mathrm{H}$ deformation of methyl groups present only on the spectrum of GalXMe (the region at $2986 \mathrm{~cm}^{-1}$ corresponding to the stretching vibration of $(\mathrm{C}-\mathrm{H}) \mathrm{CH}_{3}$ is intentionally not mentioned here due to the very low intensity and overlapping signals). The other region is 
the region of the stretching vibrations of C-O-C coupled with $\mathrm{C}-\mathrm{C}$ present at $927 \mathrm{~cm}^{-1}$ for GalXH and at $866 \mathrm{~cm}^{-1}$ for GalXMe.

A very useful region during the analysis of two polyamides is the signal present around 1080 $\mathrm{cm}^{-1}$. This high intensity signal, corresponding to the stretching vibration of C-O-C, is present for both acetal fragments. Multiple low intensity peaks present between $1290-1260 \mathrm{~cm}^{-1}$ correspond to different stretching and skeletal $(\gamma)$ vibrational modes typical for polyamides.

\subsection{Conclusions}

The synthesis of homopolyamides from GalX diethyl esters gives promising results affording polymers with elevated glass transition temperatures. The acetal moieties are stable at elevated temperatures of the synthesis. Homopolyamides containing bio-based GalX units and aromatic diamines can be successfully synthesized by the application of the phosphorylation method. Polymerization of rigid GalX with cycloaliphatic and aromatic diamines allows for the obtainment of polyamides with glass transition temperatures above $150{ }^{\circ} \mathrm{C}$. Maldi-ToF analysis confirmed the expected structures and revealed the presence of some side products corresponding to the cyclization, ethylation of amines and selfcondensation of amines. The GalX polyamides are chemically stable in mildly acidic medium of $5 \mathrm{wt} \%$ citric acid solution, however show different stability in $90 \mathrm{wt} \%$ formic acid. Generally, the GalXH acetal moiety is resistant to the exposition to concentrated acids and the GalXMe acetal undergoes hydrolysis in those conditions. Both GalX polyamides are stable at temperatures above $300{ }^{\circ} \mathrm{C}$. The GalXMe polyamides do not show any cross-linking behavior during melt condensation, while polymerization with GalXH results in branching / cross-linking. Moreover, the GalXMe polyamides are stable for elongated periods at elevated temperatures, what makes the GalXMe homopolyamide a promising candidate for processing experiments.

\subsection{Materials and methods}

\subsubsection{Materials}

Diethyl $\quad(2,3 ; 4,5-($ di- $O$-isopropylidene)galactarate $)) \quad>99 \%, \quad$ Diethyl $\quad(2,3 ; 4,5-(\mathrm{O}-$ dimethylene)galactarate) ) $>99 \%, 2,3 ; 4,5$-(di- $O$-isopropylidene)galactaric acid $>99 \%$ and 2,3;4,5-(O-dimethylene)galactaric acid $>99 \%$ were supplied by Royal Cosun. 1,6hexamethylenediamine (HMDA) 98\%, 1,3-phenylenedimethanamine (MXD) 98\%, 5Amino-1,3,3-trimethylcyclohexanemethanamine

(IPDA) $98 \%$, 4,4'- 
methylenebis(cyclohexylamine) (PACM) 95\%, 4,4'- methylenebis(2methylcyclohexylamine) (PACM-Me) 99\%, p-phenylenediamine (PPDA) 98\% , 4,4'oxydianiline (OXDA) 97\%, citric acid, Irganox 1330, 90\% formic acid, dithranol, NaTFA and polyamide 6,12 (PA6,12) were purchased from Sigma-Aldrich and used as supplied. 1,1,1,3,3,3-hexafluoro-2-propanol (HFIP), dimethyl sulfoxide (DMSO) 99.7\% DMSO-d 6 , $\mathrm{CDCl}_{3}$, pyridine $99.5 \%$ Extra Dry, dry N-methylpirrolidone (NMP), and triphenylphosphate 99\% (dried over molecular sieves $3 \AA$ or $4 \AA$ prior to use) were purchased from Acros Organics. Calcium chloride as anhydrous powder was purchased from Merck. Tetrahydrofuran (THF), acetone, chloroform $\left(\mathrm{CHCl}_{3}\right)$, ethanol were purchased from Biosolve.

\subsubsection{Methods}

Poly(hexamethylene-2,3;4,5-O-isopropylidene-galactaramid). To a $100 \mathrm{~mL}$ three-necked round bottom flask (equipped with a vacuum-tight mechanical stirrer, a Vigreux column and a distillation condenser) diethyl 2,3;4,5-di-O-isoplopylidenegalactarate (6.93 g, $20 \mathrm{mmol})$, hexamethylene diamine $(2.75 \mathrm{~g}, 20 \mathrm{mmol})$ and Irganox 1330 as an antioxidant $(0.09 \mathrm{~g}, 0.1$ $\mathrm{wt} \%$ ) were added and slowly heated to $210^{\circ} \mathrm{C}$. After all ethanol had been fully distilled off removed, vacuum was applied for 1-3 $\mathrm{h}$. The crude product was afforded as a yellowish material.

${ }^{1} H$ NMR (300 MHz, DMSO-d 6 ) $\square$ (ppm): 6.68 (2H, -NH-, s), 4.55 (2H, -CH-O-, m), $4.36(2 \mathrm{H}$, -CH-O-, m), 4.28 (4H, - $\mathrm{CH}_{2}-\mathrm{NH}-\mathrm{t}$ ), 1.58 (4H, $\left.-\mathrm{CH}_{2}-\mathrm{CH}_{2}, \mathrm{HMDA}, \mathrm{m}\right), 1.40$ (6H, $\mathrm{CH}_{3}$, GalX, s)

${ }^{13} \mathrm{C} N M R\left(75 \mathrm{MHz}, \mathrm{DMSO}-\mathrm{d}_{6}\right) \delta(\mathrm{ppm}): 170.6$ (2C, $\left.\mathrm{C}=\mathrm{O}\right), 110.7$ (2C, O-C-O), 78.8 (2C, CH-O-), 75.5 (2C, -CH-O-), 38.7 (2C, $\left.\mathrm{CH}_{2}-\mathrm{NH}_{2}\right), 29.4$ (2C, $\left.\mathrm{CH}_{2}-\mathrm{CH}_{2}-\mathrm{NH}_{2}\right), 27.1$ (2C, C$\left.\mathrm{CH}_{3}\right), 26.5$ (2C, $\left.\mathrm{CH}_{2}-\mathrm{CH}_{2}-\mathrm{CH}_{2}-\mathrm{NH}_{2}\right), 25.8\left(2 \mathrm{C}, \mathrm{C}-\mathrm{CH}_{3}\right)$,

Poly(hexamethylene-2,3;4,5-O-hexamethylene-galactaramid) was synthesized according to the previously described procedure.

${ }^{1} H$ NMR (300 MHz, DMSO-d $)_{6} \delta(p p m): 6.74(2 \mathrm{H},-\mathrm{NH}-, \mathrm{s}), 5.21\left(2 \mathrm{H}, \mathrm{O}-\mathrm{CH}_{2}-\mathrm{O}, \mathrm{s},\right), 4.99$ $\left(2 \mathrm{H}, \mathrm{s}, \mathrm{O}-\mathrm{CH}_{2}-\mathrm{O}\right), 4.60$ (2H, -CH-O-, m), 4.32 (2H, -CH-O-, m), 3.27 (4H, -CH $\left.\mathrm{CH}_{2} \mathrm{NH}-\mathrm{t}\right), 1.58$ $\left(4 \mathrm{H},-\mathrm{CH}_{2}-\mathrm{CH}_{2}-\mathrm{NH}, \mathrm{m}\right), 1.26\left(4 \mathrm{H},-\mathrm{CH}_{2}-\mathrm{CH}_{2}-\mathrm{CH}_{2}-\mathrm{NH}, \mathrm{m}\right)$

${ }^{13} \mathrm{C} \mathrm{NMR}\left(75 \mathrm{MHz}, \mathrm{DMSO}-d_{6}\right) \delta(\mathrm{ppm}): 169.8(2 \mathrm{C}, \mathrm{C}=\mathrm{O}), 95.9\left(2 \mathrm{C}, \mathrm{O}-\mathrm{CH}_{2}-\mathrm{O}\right), 79.6(2 \mathrm{C}$, CH-O-), 75.2 (2C, -CH-O-), 38.7 (2C, $\left.\mathrm{CH}_{2}-\mathrm{NH}_{2}\right), 29.3\left(2 \mathrm{C}, \mathrm{CH}_{2}-\mathrm{CH}_{2}-\mathrm{NH}_{2}\right), 26.4\left(2 \mathrm{C}, \mathrm{CH}_{2}-\right.$ $\mathrm{CH}_{2}-\mathrm{CH}_{2}-\mathrm{NH}_{2}$ ) 
Phosphorylation. The phosphorylation reaction was performed according to the procedure presented by Zhao et al. ${ }^{16}$, which is originally based on the procedure developed by Higashi et al. ${ }^{28}$.

Poly(paraphenylene-2,3;4,5-O-isopropylidene-galactaramid) To a completely dried 100-mL, three-necked flask equipped with a mechanical stirrer and a condenser were charged 2,3;4,5$O$-isopropylidene-galactaric acid (1.45 g, $5 \mathrm{mmol})$, p-phenylenediamine $(0.54 \mathrm{~g}, 5 \mathrm{mmol})$, calcium chloride $(1.25 \mathrm{~g})$, triphenylphosphite $(2.8 \mathrm{~mL}, 10 \mathrm{mmol})$, pyridine $(2.5 \mathrm{~mL})$, and NMP (10.6 mL) under nitrogen flow. The mixture was mechanically stirred and heated to $80^{\circ} \mathrm{C}$. After the mixture was completely dissolved, the mixture was heated while stirring under nitrogen at $100^{\circ} \mathrm{C}$ for $3 \mathrm{~h}$ and at $110^{\circ} \mathrm{C}$ for $3 \mathrm{~h}$, respectively. The resulting viscous polymer solution was poured slowly into $300 \mathrm{~mL}$ of stirring methanol resulting in a fiberlike precipitate, which was collected by filtration, washed thoroughly with methanol and hot water, and dried at $120^{\circ} \mathrm{C}$ for $24 \mathrm{~h}$ in vacuo.

${ }^{1} H$ NMR (300 MHz, DMSO-d $) \delta$ (ppm): 10.04 (2H, -NH-, s), 7.63 (4H, Ph, s,), 4.63 (2H, $\mathrm{CH}-\mathrm{O}-, \mathrm{m}), 4.58$ (2H, -CH-O-, m), $1.45\left(6 \mathrm{H},-\mathrm{CH}_{3}, \mathrm{~s}\right), 1.39\left(6 \mathrm{H},-\mathrm{CH}_{3}, \mathrm{~s}\right)$

Low molecular weight poly(paraphenylene-2,3;4,5-O-isopropylidene-galactaramid). The polymer was synthesized via the phosphorylation method ${ }^{16}$ using 2,3;4,5-O-isopropylidenegalactararic acid and p-phenylenediamine with a molar ratio of carboxylic groups to amine groups of 0.010:0.0082. The low molecular weight polymer was precipitated in methanol at room temperature and filtered, affording the fraction PA(PPDA-GalXMe). The filtrate was left in the freezer overnight to precipitate out residual low molecular weight product, which was soluble in methanol at room temperature. The solution was filtered and the second fraction was isolated as (PA(PPDA-GalXMe).

\subsubsection{Characterization}

${ }^{1} \mathrm{H}$ - and ${ }^{13} \mathrm{C}-\mathrm{NMR}$ spectra were recorded on a Bruker Avance 300 at $300 \mathrm{MHz}$ in $\mathrm{CDCl}_{3}$ or in DMSO- $\mathrm{d}_{6}$. Chemical shifts are presented in parts per million $(\delta)$ relative to DMSO- $\mathrm{d}_{6}(2.50$ ppm in ${ }^{1} \mathrm{H}$ - and $39.51 \mathrm{ppm}$ in ${ }^{13} \mathrm{C}-\mathrm{NMR}$ respectively) or $\mathrm{CHCl}_{3}-\mathrm{d}_{1}\left(7.26 \mathrm{ppm}\right.$ in ${ }^{1} \mathrm{H}$ - and 77.16 ppm in ${ }^{1} \mathrm{C}-\mathrm{NMR}$ respectively). The resonance multiplicities are described as s (singlet), $\mathrm{d}$ (doublet), $\mathrm{t}$ (triplet) or $\mathrm{m}$ (multiplet).

Molecular weight of polyamides was determined via Gel Permeation Chromatography (GPC). The polymers were dissolved in 1,1,1,3,3,3-hexafluoroisopropanol (HFIP) with 0,019\% NaTFA salt. The sample for GPC measurement was prepared by dissolving $5.0 \mathrm{mg}$ of the polymer in $1.5 \mathrm{ml}$ of the solvent. The solutions were filtered over a $0.2 \mu \mathrm{m}$ PTFE syringe 
filter before injection. The GPC apparatus was calibrated with poly (methyl methacrylate) standards. Two PFG combination medium microcolumns with $7 \mu \mathrm{m}$ particle size $(4.6 \times 250$ $\mathrm{mm}$, separation range 100-1.000.000 Da) and precolumn PFG combination medium with 7 $\mu \mathrm{m}$ particle size $(4.6 \times 30 \mathrm{~mm})$ with Refractive Index detector $(\mathrm{RI})$ were used in order to determine molecular weight and dispersities.

Melting temperatures were measured by a Mettler Toledo MP90 Melting Point System with a heating rate of $10^{\circ} \mathrm{C} / \mathrm{min}$.

The thermal stability of the prepared polyamides and monomers were determined using TGA (TA Instruments Q500). Approximately $10 \mathrm{mg}$ of the material was heated at $10^{\circ} \mathrm{C} / \mathrm{min}$ from $25^{\circ} \mathrm{C}$ to $700{ }^{\circ} \mathrm{C}$ in a nitrogen atmosphere. The isothermal tests were prepared at $210{ }^{\circ} \mathrm{C}$ and $280{ }^{\circ} \mathrm{C}$ with a heating rate of $10^{\circ} \mathrm{C} / \mathrm{min}$ from $25^{\circ} \mathrm{C}$ to the desired temperature.

Rheological properties of polymers were measure on an Anton Paar Rheometer MRC702 TwinDrive with a convection heating system CTD450 with plate diameter $5 \mathrm{~mm}$. The viscosity measurement was conducted between 60 and $250{ }^{\circ} \mathrm{C}$. A sample was placed on the rheometer plate at $250{ }^{\circ} \mathrm{C}$ and then the temperature was decreased till $60{ }^{\circ} \mathrm{C}$ at a cooling rate $5{ }^{\circ} \mathrm{C} / \mathrm{min}$ with an angular frequency $1 \mathrm{rad} / \mathrm{s}$. The frequency sweep was recorded for the polymers every $20{ }^{\circ} \mathrm{C}$ starting from slightly above the $T_{g}$ up to $240{ }^{\circ} \mathrm{C}$ and based on the shift factors the plots were shifted to the reference temperature of $240{ }^{\circ} \mathrm{C}$ or in case of PA(IPDA, GalXMe) to $220^{\circ} \mathrm{C}$.

Table 2-5 The GPC characterization of polymers used for the rheology.

\begin{tabular}{cccc}
\hline Entry & Composition & $\begin{array}{c}\boldsymbol{M}_{\boldsymbol{w}, \boldsymbol{G P C}} \\
\left(\mathbf{k g} \cdot \mathbf{m o l}^{\mathbf{- 1}} \mathbf{)}\right.\end{array}$ & $\boldsymbol{Ð}_{\boldsymbol{G P C}}$ \\
\hline PA2' & PA(DDDA,GalXH) & 61.1 & 4.7 \\
PA3, & PA(MXD,GalXH) & 65.8 & 4.7 \\
PA10' & PA(DDDA,GalXMe) & 56.7 & 2.7 \\
PA11' & PA(MXD,GalXMe) & 32.3 & 1.7 \\
PA12, & PA(IPDA,GalXMe) & 28.9 & 1.7 \\
\hline
\end{tabular}

The DSC thermograms were analyzed using a TA Instruments DSC Q1000. Before DSC measurement, the samples were dried in a vacuum oven at $80{ }^{\circ} \mathrm{C}$. For measurement of the glass transition temperature $\left(T_{\mathrm{g}}\right)$, the polymers were first subjected to a heating and cooling cycle. Then the samples were heated from $25{ }^{\circ} \mathrm{C}$ to $250{ }^{\circ} \mathrm{C}$ at a scanning rate of $10{ }^{\circ} \mathrm{C} / \mathrm{min}$. 
Indium and Sapphire were used for temperature calibration. The $T_{g}$ of each polymer was determined as the temperature at the midpoint of the transition.

Hydrolysis tests were performed after 60 days of conditioning polymer powder in $5 \%$ citric acid or $2 \mathrm{~h}$ in $90 \%$ formic acid at room temperature.

Matrix-assisted laser desorption/ionization time-of-flight (Maldi-ToF) mass spectra were recorded on a Bruker UltrafleXtreme spectrometer with a $355 \mathrm{~nm} \mathrm{Nd:Yag} \mathrm{laser}(2 \mathrm{kHz}$ repetition pulse/Smartbeam-IITM) and a grounded steel plate. All mass spectra were obtained in reflector mode. Dithranol $(20 \mathrm{mg} / \mathrm{mL}$ in HFIP) was used as a matrix, NaTFA $(5 \mathrm{mg} / \mathrm{mL}$ HFIP) was used as a cationating agent, and polymer samples were dissolved in HFIP (1 $\mathrm{mg} / \mathrm{mL}$ ). The applied ratio of polymer:matrix:salt was 20:200:10. Poly(ethylene glycol) standards with $M_{\mathrm{n}}$ equal to 5000,10000 and $15000 \mathrm{~g} / \mathrm{mol}$ were used for calibration. All data were processed using the FlexAnalysis (Bruker Daltonics) software package. 


\section{REFERENCES}

1. M. Winnacker and B. Rieger, Macromol. Rapid Commun., 2016, 37, 1391-1413.

2. A. Gandini and T. M. Lacerda, Prog. Polym. Sci., 2015, 48, 1-39.

3. C. Lavilla, A. Alla, A. Martínez de Ilarduya, E. Benito, M. G. García-Martín, J. A. Galbis and S. Muñoz-Guerra, J. Polym. Sci., Part A: Polym. Chem., 2012, 50, 3393-3406.

4. E. Gubbels, C. Lavilla, A. M. de Ilarduya, B. A. J. Noordover, C. E. Koning and S. MuñozGuerra, J. Polym. Sci., Part A: Polym. Chem., 2014, 52, 164-177.

5. C. Lavilla, A. Alla, A. M. de Ilarduya, E. Benito, M. G. Garcia-Martin, J. A. Galbis and S. Munoz-Guerra, Biomacromolecules, 2011, 12, 2642-2652.

6. C. Lavilla, A. Alla, A. Martínez de Ilarduya, E. Benito, M. G. García-Martín, J. A. Galbis and S. Muñoz-Guerra, J. Polym. Sci., Part A: Polym. Chem., 2012, 50, 1591-1604.

7. S. Muñoz-Guerra, C. Lavilla, C. Japu and A. Martínez de Ilarduya, Green Chem., 2014, 16, 1716-1739.

8. C. Lavilla and S. Muñoz-Guerra, Green Chem., 2013, 15, 144-151.

9. C. Lavilla and S. Muñoz-Guerra, Polym. Degrad. Stab., 2012, 97, 1762-1771.

10. K. Butler and D. R. Lawrance, GB Pat., GB Patent 750,822, 1956.

11. W. A. P. Black, E. T. Dewar and D. Rutherford, U.S. Patent 3,225,012, 1965.

12. C. Rosu, I. I. Negulescu, R. Cueto, R. Laine and W. H. Daly, J. Macromol. Sci., Part A Pure Appl. Chem., 2013, 50, 940-952.

13. A. Rodriquez-Galan, J. J. Bou and S. Munoz-Guerra, J. Polym. Sci., Part A: Polym. Chem., 1992, 30, 713-721.

14. M. E. Rogers and T. E. Long, Synthetic methods in step-growth polymers., A John Wiley \& Sons, inc., Hoboken, New Jersey, 2003.

15. P. W. Morgan and S. L. Kwolek, Macromolecules, 1974, 8, 104-111.

16. J. Zhao, H. Xu, J. Fang and J. Yin, J. Appl. Polym. Sci., 2012, 126, 244-252.

17. G. Thouas and Q. Chen, Biomaterials: A Basic Introduction., Taylor \& Francis Group, LLC., 2015.

18. P. G. M. Wuts and T. W. Greene, Greene's Protective Groups in Organic Synthesis, John Wiley \& Sons, Inc., Fourth edn., 2007.

19. N. Ogata, K. Sanui, K. Hosoda and H. Nakamura, J. Polym. Sci., Polym. Chem. Ed., 1977, 15, 1523-1526.

20. N. Ogata, K. Sanui, T. Ohtake and H. Nakamura, Polym. J., 1979, 11, 827-833.

21. S. Amslinger, A. Hirsch and F. Hampel, Tetrahedron, 2004, 60, 11565-11569.

22. C. Hahn, H. Keul and M. Möller, Polym. Int., 2012, 61, 1048-1060.

23. D. W. van Krevelen and K. Nijenhuis, Properties of Polymers:their correlation with chemical structure: their numerical estimation and prediction from additive group contributions., Elsevier, Amsterdam, 2009.

24. L. Franco and J. Puiggalí, J. Polym. Sci., Part B: Polym. Phys., 1995, 33, 2065-2073.

25. I. Takashi, Jpn. J. Appl. Phys, 1976, 15, 2295-2306.

26. Y. Yoshioka and K. Tashiro, Polymer, 2003, 44, 7007-7019.

27. J. Wu, L. Jasinska-Walc, D. Dudenko, A. Rozanski, M. R. Hansen, D. van Es and C. E. Koning, Macromolecules, 2012, 45, 9333-9346.

28. F. Higashi, S. I. Ogata and Y. Aoki, J. Polym. Sci., Polym. Chem. Ed., 1982, 20, 2081-2087. 


\section{Chapter 3 STRUCTURE-PROPERTY RELATIONS IN GALX- BASED MONOMERS AND POLYMERS}

Abstract

This chapter focuses on the exploration of structurally different acetals of diethyl galactarate. Cyclohexilidyne, benzylidene and p-fluorobenzylidene acetals are synthesized and the properties of polyamides made thereof are investigated along with i-propylidene and methylene acetals. The structure of the acetal highly influences the glass transition temperature and water absorption and additionally it delivers a method to introduce new functionalities into polymers. The reactivity of the different acetalized monomers towards diamines greatly depends on the chemical variation of the a carbon substituent of ester. Compared to non-substituted linear monomer, increased reactivity was observed for the different biacetal derivatives of galactaric diethyl ester. This kinetic property allows conducting a polymerization at lower temperatures than normally expected for polyamides. The structures of the new monomers and polyamides thereof are elucidated by NMR and Maldi-ToF. 



\subsection{Introduction}

Carbohydrate-derived compounds hold a great potential as monomers for polymer production, particularly when their production does not compete with the plantations designated for food production. ${ }^{1,2}$ Diethyl galactarate (1, Figure 3-1) is an example of such a molecule obtained from sugar beet pulp, a waste product from sugar production. Typically, in comparison with common monomers, sugar-derived monomers contain functionalities in their backbone like hydroxyl groups. Those molecules may be polymerized directly using sophisticated methods

3-5 or hydroxyl functionalities can be protected or further functionalized by acetal formation prior to the polymerization process. Up to date, polycondensation of such protected polyhydroxy moieties (ester, acid or diol derivatives) was applied in order to synthesize polyesters $^{6-10}$, polyamides $^{5,}{ }^{11-13}$, polycarbonates ${ }^{14}$ and polyurethanes ${ }^{15}$, 16, using the protection to reduce the amount of functional groups in the multifunctional compound. This chapter primarily will focus on polyamides from cyclic acetal protected diethyl galactarate (GalX). Compared to polyester synthesis via melt condensation, polyamide synthesis is more challenging due to the higher reaction temperatures required. This becomes even more challenging in case of monomers like GalX that are prone to thermally induced deprotection of the hydroxyl groups. The current literature on polymerization of cyclic biacetalyzed galactarate derivatives solves this issue by performing polycondensations in solution or via interfacial polymerization at relatively low temperatures. ${ }^{5,13,17-19}$ We will, however, apply an environmentally more benign melt polymerization method via amidation of GalX diethyl esters with 1,6-hexamethylene diamine. This strategy is previously described in Chapter 2 for the polymerization of diethyl 2,3:4,5-di-O-isopropylidene-galactarate (GalXMe, 2a) and diethyl 2,3:4,5-di-O-methylene-galactarate (GalXH, $2 \mathbf{b}$ ) with diamines. ${ }^{20}$ In this chapter the effect of different protecting groups on the properties of the resulting polyamides is investigated. The target of this part is to explore the opportunities brought by different acetal protections (cyclohexylidene $\mathbf{2 c}$, benzylidene $\mathbf{2 d}$ and fluorobenzylidene $\mathbf{2 e}$, next to $\mathbf{2 a}$ and $\mathbf{2 b}$ as reference (see Figure 3-1)) in diethyl galactarate monomers, since this can significantly change the thermal properties and the hydrophilicity of the polymers derived thereof (respectively polyamides PA3, PA4 and PA5, with PA1 and PA2 as reference, Figure 3-1). Moreover, the reactivity of the different GalX monomers will be compared with the reactivity of a typical non-carbohydrate-based linear monomer, namely the diethyl ester of adipic acid (2f). Up to now, this is the first report about the synthesis and polymerization of GalXHex (2c) and GalXBnF (2e), while GalXBn (2d) has been made before, but was only polymerized 
via environmentally less preferable interfacial polymerization. ${ }^{19}$ Eventually this strategy will allow us to create a tool that enables the design of random functionalized GalX copolymers with a controlled amount of different functionalities.

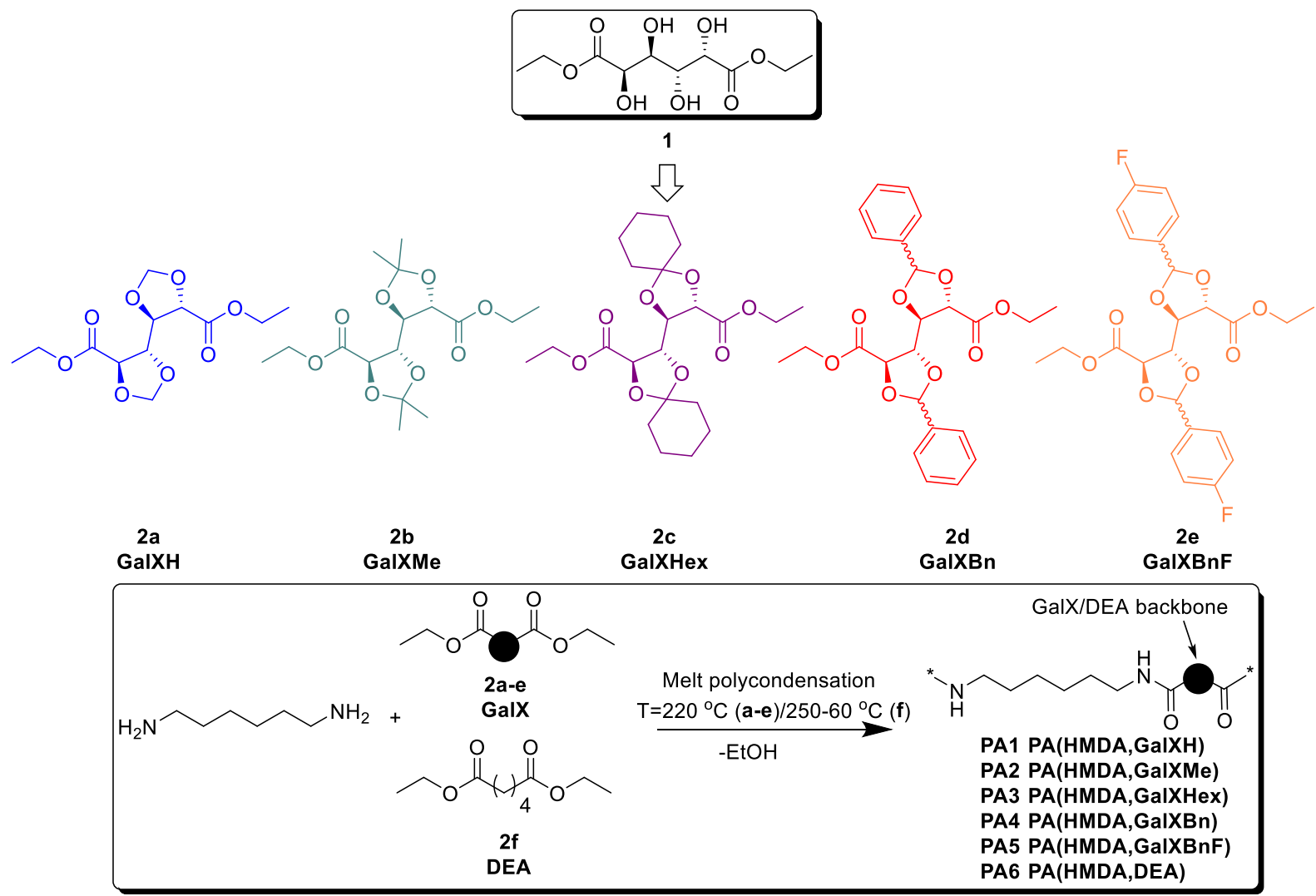

Figure 3-1 Synthetic approach towards diethyl galactarate derived polyamides.

\subsection{The synthesis of GalX derivatives and PAs therefrom}

In previous chapter the synthesis of polyamides from two acetals of GalX, namely diethyl 2,3:4,5-di-O-methylene-galactarate $(\mathrm{GalXH}, \mathbf{2 a})$ and diethyl 2,3:4,5-di- $O$-isopropylidenegalactarate (GalXMe, 2b) was reported. ${ }^{20}$ New acetal derivatives (2c-2d) of diethyl galactarate (1) were prepared by the acetalization of the hydroxyl groups in a presence of an acidic catalyst ( $p$-toluene sulfonic acid, p-TSA). Acetalization of the hydroxyl groups was performed with an aldehyde $(\mathbf{2} \mathbf{a}, \mathbf{2 b}, \mathbf{2 e})$ or via transacetalization with the dimethyl acetal of the corresponding aldehyde/ketone $(\mathbf{2 c}, \mathbf{2 d})$. The latter resulted in the release of methanol which together with heating and acidic catalysis also stimulated the transesterification reaction of the diethylester end groups, resulting in diacetalized galactarates with a mixture of dimethyl and diethyl ester end groups. The transesterification does not have a major influence on the polymerization as long as the molar ratio of ester groups to amine groups is $1: 1$. The presence of the dimethyl ester, however, might cause an increased alkylation rate of 
the amine groups. ${ }^{21}$ Subsequently, the monomers were polymerized by polycondensation of diester galactarates with hexamethylenediamine (HMDA) resulting in a series of polyamides (PA1-PA5). The polymer from diethyl adipate (DEA) and HMDA was also synthesized as a reference polymer (PA6); however this polymerization requires $30-40{ }^{\circ} \mathrm{C}$ higher reaction temperatures that for GalX derivatives (Table 3-1). The corresponding NMR spectra of the new diethyl galactarate monomers $\mathbf{2 c - 2 e}$ and polyamides therefrom are available in the Appendix 2. The molecular weight of the polyamides varied between $8.7-24 \mathrm{~kg} \cdot \mathrm{mol}^{-1}$ (Table $3-1)$.

Table 3-1 Polyamides prepared with different GalX derivatives together with analytical data including $T_{g}, M_{n}$, $Ð$ and thermal stability (TGA).

\begin{tabular}{|c|c|c|c|c|c|c|c|c|}
\hline & PA composition & $\begin{array}{l}T_{\text {reaction }} \\
\left({ }^{\circ} \mathrm{C}\right)\end{array}$ & $\begin{array}{l}T_{g}{ }^{a} \\
\left({ }^{\circ} \mathrm{C}\right)\end{array}$ & $\begin{array}{l}M_{n}{ }^{b} \\
\left(\mathrm{~kg}^{\prime} \cdot \mathrm{mol}^{-1}\right)\end{array}$ & $\boldsymbol{\Xi}^{b}$ & $\begin{array}{l}\text { Mol \%c } \\
\text { GalX:HMDA }\end{array}$ & $\begin{array}{l}T_{10 \%}{ }^{d} \\
\left({ }^{\circ} \mathrm{C}\right)\end{array}$ & $\begin{array}{l}T_{\max }{ }^{t} \\
\left({ }^{\circ} \boldsymbol{C}\right)\end{array}$ \\
\hline$P A 1$ & $\begin{array}{l}\text { HMDA- } \\
\text { GalXH }\end{array}$ & 220 & 73 & 24 & 4.3 & $50.4: 49.6$ & 367 & $402 / 443$ \\
\hline$P A 2$ & $\begin{array}{l}\text { HMDA- } \\
\text { GalXMe }\end{array}$ & 220 & 82 & 23 & 2.5 & $50.9: 49.1$ & 374 & $398 / 443$ \\
\hline$P A 3$ & $\begin{array}{l}\text { HMDA- } \\
\text { GalXHex }\end{array}$ & 220 & 87 & 17 & 2.0 & $50.7: 49.3$ & 369 & $403 / 440$ \\
\hline$P A 4$ & $\begin{array}{l}\text { HMDA- } \\
\text { GalXBn }\end{array}$ & 220 & 97 & 10 & 2.2 & $50.8: 49.2$ & 344 & $365 / 446$ \\
\hline$P A 5$ & $\begin{array}{l}\text { HMDA- } \\
\text { GalXBnF }\end{array}$ & 220 & 95 & 8.7 & 3.8 & $50.8: 49.2$ & 366 & $397 / 466$ \\
\hline PA6 & $\begin{array}{l}\text { HMDA- } \\
\text { DEA }^{\mathrm{e}}\end{array}$ & $250-260$ & $50^{22}$ & 23 & 4.4 & $48.7: 51.3$ & 400 & 434 \\
\hline
\end{tabular}

adetermined by DSC betermined by GPC ${ }^{~}$ Determined by ${ }^{1} \mathrm{H}$ NMR analysis (see Appendix A) ${ }^{d}$ Determined by TGA ${ }^{\mathrm{e}} T_{\mathrm{m}}=253{ }^{\circ} \mathrm{C}, \Delta H_{\mathrm{m}}=123 \mathrm{~J} / \mathrm{g}$

The molar ratio of the monomers in the product was in all cases similar to the molar ratio in the feed (50:50), with the balance shifted slightly towards an excessive amount of GalX (Table 3-1). It was already reported that GalXH (2a) and HMDA give polymers with high dispersity (elevated $\mathrm{M}_{\mathrm{w}}$ ) with tendency to cross-link at high conversions. The thermally induced deprotection of the hydroxyl groups in the GalX backbone is the most challenging factor during polyamide synthesis in the melt; however not in each case the deprotection leads to the cross-linking. The cross-linking depends on the accessibility of the hydroxyl groups, which is limited with more bulky structures of the neighboring remaining acetal. This phenomenon explains why polyamide PA1 without a bulky acetal has the highest dispersity of all polyamides in Table 3-1. Using the fact that the GalX ester is activated by the presence of oxygen on $\alpha$ and $\beta$ carbon ${ }^{23,24}$ compared to the aliphatic counterparts like DEA (2f) we can apply relatively low reaction temperatures $\left(220^{\circ} \mathrm{C}\right)$ compared to typical temperatures for polyamide synthesis $\left(250-280{ }^{\circ} \mathrm{C}\right)$. Moreover, gradual heating of the monomers is a useful 
tool to control thermal deprotection and helps to almost entirely eliminate side reactions in case of GalX monomers with a bulky acetal protection. The GPC traces collected for all the prepared polyamides are available in Figure 3-2.

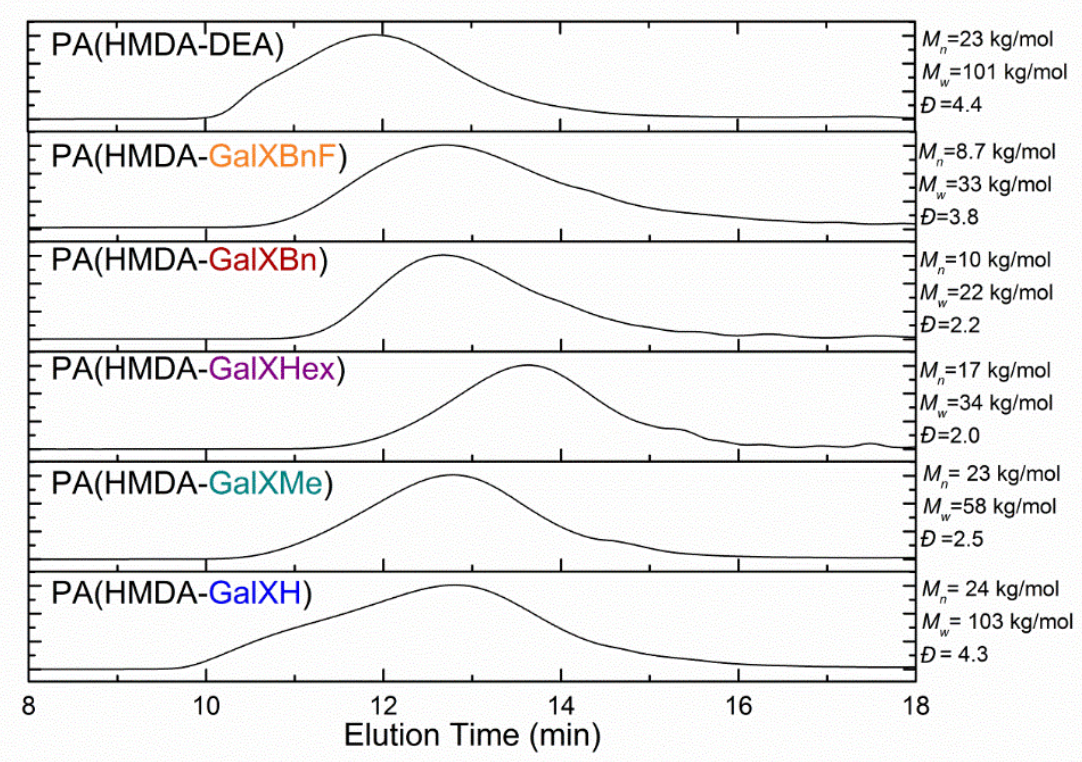

Figure 3-2 GPC traces of polyamides 3a-3f recorded in HFIP with PMMA standards (RI detection).

\subsection{Properties of polyamides made with Different GalX Derivatives}

\subsubsection{Maldi-ToF Analysis}

Typically, polyamides prepared via ester-amide exchange chemistry can be terminated with an ethyl ester (end group - $\mathrm{OCH}_{2} \mathrm{CH}_{3}$ ), an amine (end group - $\mathrm{H}$ ) or have no end groups when chain ends react with each other, leading to the formation of cyclic polymeric chains (corresponding structures are presented in Figure 2-6 in Chapter 2). The PA(HMDA,DEA) spectrum (Figure 3-3 (a)) shows multiple distributions, among which the predominant distribution $\alpha$ corresponds to chains terminated with two ester groups on both ends $\left(\mathrm{X}_{1}\right.$ $\left.\mathrm{OCH}_{2} \mathrm{CH}_{3}, \mathrm{X}_{2}-\mathrm{C}_{6} \mathrm{H}_{8} \mathrm{O}_{2}-\mathrm{OCH}_{2} \mathrm{CH}_{3}\right)$. Distribution $\beta$ is of weak intensity and accounts for polymer terminated with an amine group $\left(\mathrm{X}_{2}-\mathrm{H}\right)$ and an ethyl ester group $\left(\mathrm{X}_{1}-\mathrm{OCH}_{2} \mathrm{CH}_{3}\right)$. Side reactions like (di)ethylation/(di)methylation of the amine groups by the monomeric ester group ${ }^{20,25,26}$ contribute to distribution $\delta$ for the ethylated amine end groups $\left(\mathrm{X}_{1}-\mathrm{OCH}_{2} \mathrm{CH}_{3}\right.$, $\left.\mathrm{X}_{2}-\mathrm{CH}_{2} \mathrm{CH}_{3}\right)$ and $\varepsilon$ for diethylated amine end groups $\left(\mathrm{X}_{1}-\mathrm{OCH}_{2} \mathrm{CH}_{3}, \mathrm{X}_{2}-\mathrm{C}_{12} \mathrm{H}_{21} \mathrm{~N}_{2} \mathrm{O}_{2}-\right.$ $\left.\left(\mathrm{CH}_{2} \mathrm{CH}_{3}\right)_{2}\right)$. The last distribution on the spectrum is the cyclic distribution $\gamma\left(\mathrm{X}_{1}-\mathrm{N} / \mathrm{A}\right.$ (not applicable), $\left.\mathrm{X}_{2}-\mathrm{N} / \mathrm{A}\right)$. 
The Maldi-ToF spectra of GalX polyamides (Figure 3-3 (b), (c) and (d)) confirm a successful incorporation of GalX derivatives into polymer backbone. The spectra show analoguous distibutions as PA(HMDA,DEA), however the observed ratio of the end groups is slightly altered. The predominant distribution in the lower molecular weight range is cyclic distribution $\gamma$, whereas at molecular weights above $1500 \mathrm{Da}$ linear distribution $\alpha$ with both ethyl ester end groups $\left(\mathrm{X}_{1}-\mathrm{OCH}_{2} \mathrm{CH}_{3}, \mathrm{X}_{2}-\mathrm{C}_{18} \mathrm{H}_{24} \mathrm{O}_{6}-\mathrm{OCH}_{2} \mathrm{CH}_{3}\right)$ becomes predominant.

$$
\begin{aligned}
& \alpha: \mathrm{X}_{1}-\mathrm{OCH}_{2} \mathrm{CH}_{3} \mathrm{X}_{2}-\text { diacid unit-OCH} \mathrm{CH}_{2} \mathrm{CH}_{3} \\
& \beta: \mathrm{X}_{1}-\mathrm{OCH}_{2} \mathrm{CH}_{3} \mathrm{X}_{2}-\mathrm{H} \\
& \gamma: \mathrm{X}_{1}-\mathrm{N} / \mathrm{A} \mathrm{X}_{2}-\mathrm{N} / \mathrm{A} \text { (cyclic distribution) } \\
& \delta: \mathrm{X}_{1}-\mathrm{OCH}_{2} \mathrm{CH}_{3} \mathrm{X}_{2}-\mathrm{CH}_{2} \mathrm{CH}_{3} \\
& \varepsilon: \mathrm{X}_{1}-\mathrm{OCH}_{2} \mathrm{CH}_{3} \mathrm{X}_{2}-\mathrm{RU}-\mathrm{H}-\left(\mathrm{CH}_{2} \mathrm{CH}_{3}\right)_{2}
\end{aligned}
$$

a.
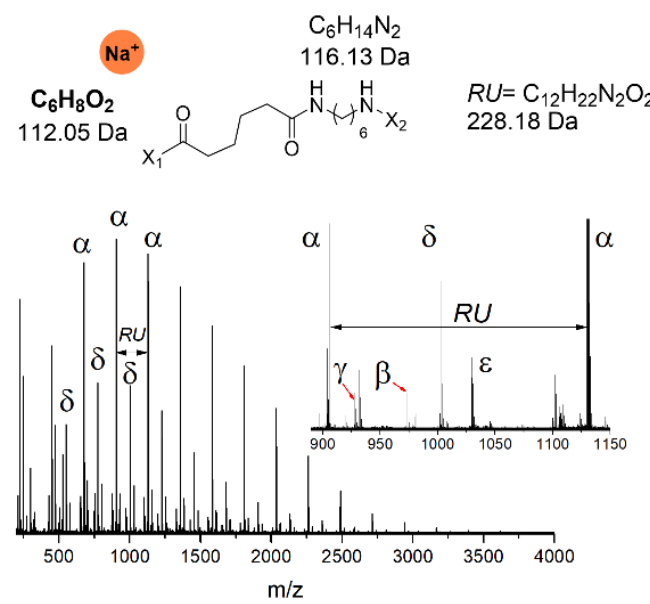

c.

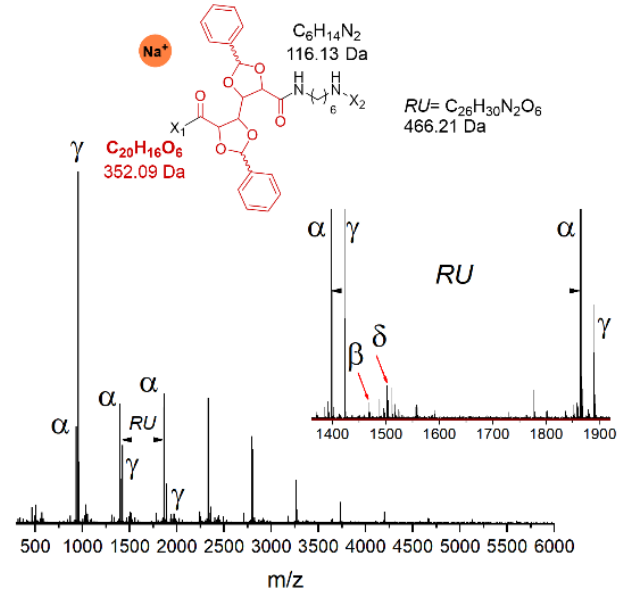

b.

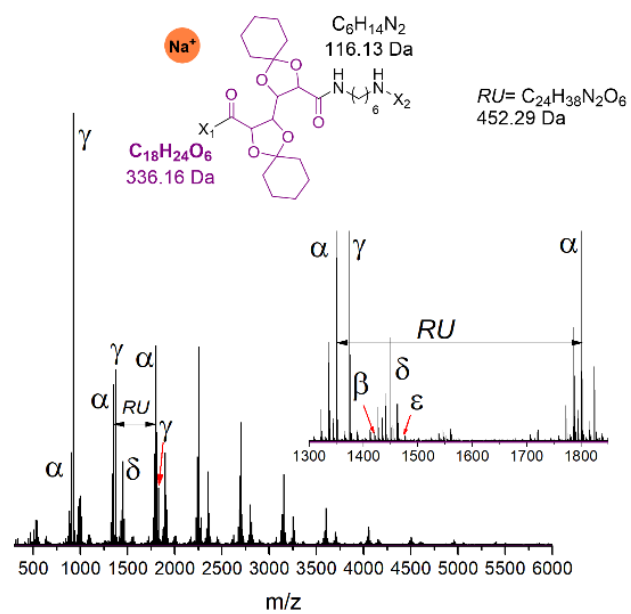

d.

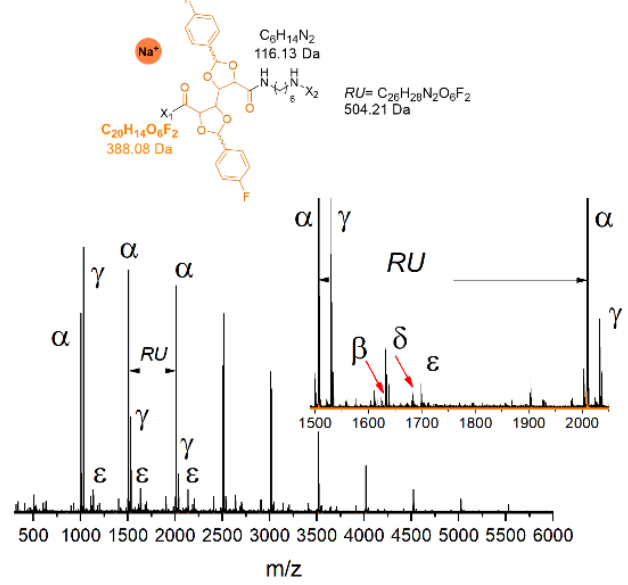

Figure 3-3 Maldi-Tof results for a. PA(HMDA,DEA) (M,GPC $\left.=3.3 \mathrm{~kg} \cdot \mathrm{mol}^{-1}, Ð=2.8\right)$, b. PA(HMDA, GalXHex) $\left(M_{n, G P C}=14 \quad \mathrm{~kg} \cdot \mathrm{mol}^{-1}, \quad D=2.7\right) \quad$ c. $\quad P A(H M D A, G a l X B n) \quad\left(M_{n, G P C}=7.5 \quad \mathrm{~kg} \cdot \mathrm{mol}^{-1}, \quad D=1.9\right) \quad$ and $\quad d$. $P A(H M D A, G a l X B n F)\left(M_{n, G P C}=4.5 \mathrm{~kg} \cdot \mathrm{mol}^{-1}, D=3.1\right)$ recorded in reflectron mode in dithranol as a matrix and NaTFA as a salt. 


\subsubsection{Thermal Properties}

The thermal properties of the GalX based polyamides were investigated by means of DSC and compared to the thermal behavior of polymer from DEA and HMDA (PA6) ( $T_{\mathrm{g}}$ values in Table 3-1 and traces present in Figure 3-4). PA(HMDA,DEA) is a semicrystalline material with a poorly distinguishable $T_{\mathrm{g}}$, which is located around $50{ }^{\circ} \mathrm{C} .{ }^{22}$ Polyamides PA1-PA5 containing GalX derivatives are all amorphous and transparent with elevated glass transition temperatures compared to the aliphatic PA(HMDA,DEA). By modification of the acetal structure, the $T_{\mathrm{g}}$ of the polymers can be efficiently tuned from $73{ }^{\circ} \mathrm{C}$ for the simplest formaldehyde protected GalX (PA1) to even $97{ }^{\circ} \mathrm{C}$ for benzaldehyde protected GalX (PA4).

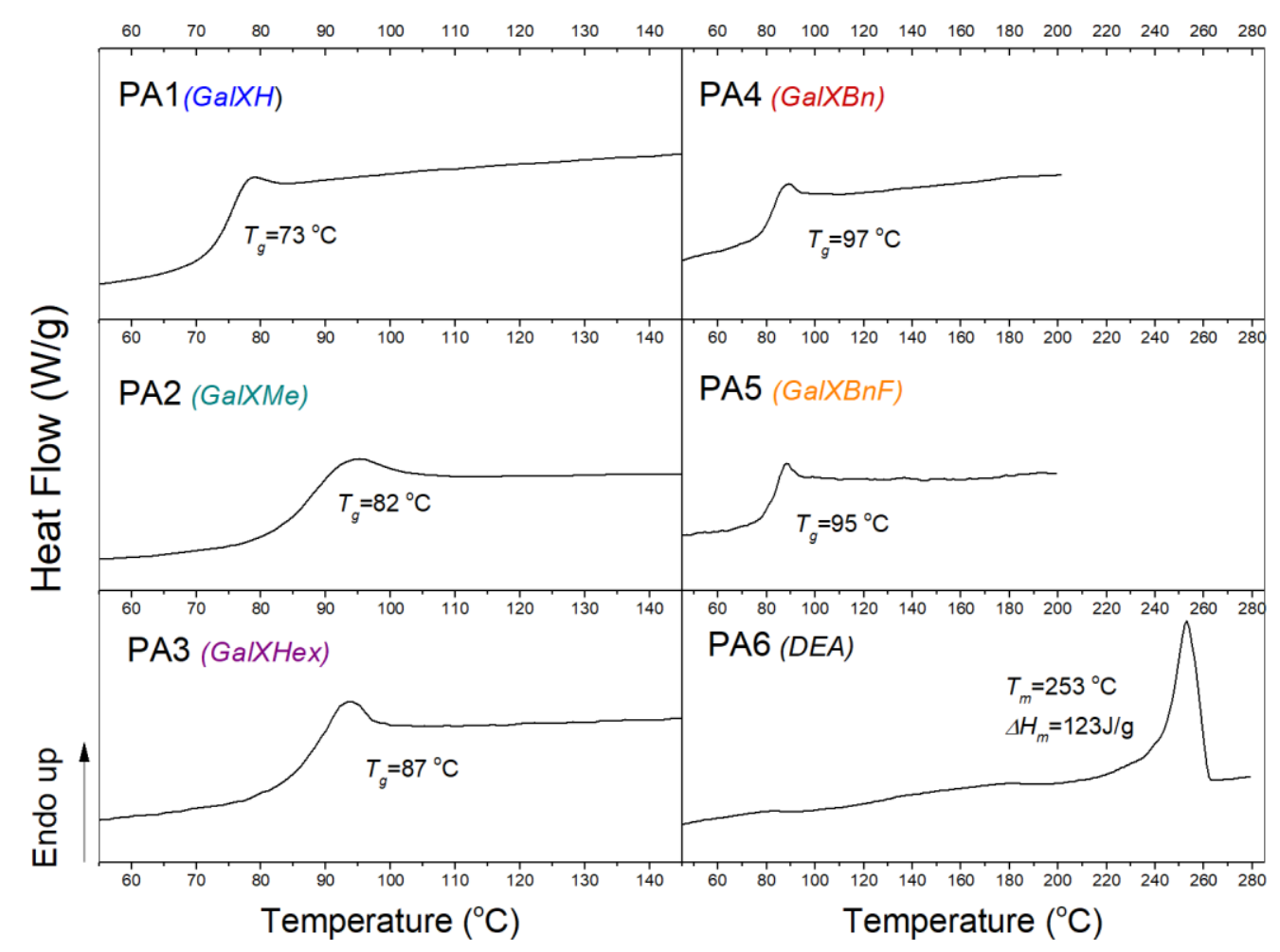

Figure 3-4 DSC traces of PA1-PA6 based on HMDA as diamine collected from second heating cycle at heating rate $10^{\circ} \mathrm{C} / \mathrm{min}$.

\subsubsection{The Reactivity of the Carboxyl Group}

The kinetics of the GalX (2a-2e) and DEA (2f) consumption during melt polycondensation revealed that GalX esters with an electronegative oxygen atom in the $\alpha$ and $\beta$ position are activated towards diamines compared to aliphatic DEA (Figure 3-5). The substantial difference in kinetics between DEA and GalX is most pronounced at the beginning of the 
reaction. After 2 minutes reaction time the consumption of DEA was only $4.8 \%$ whereas for all GalX derivatives exceeded $50 \%$.
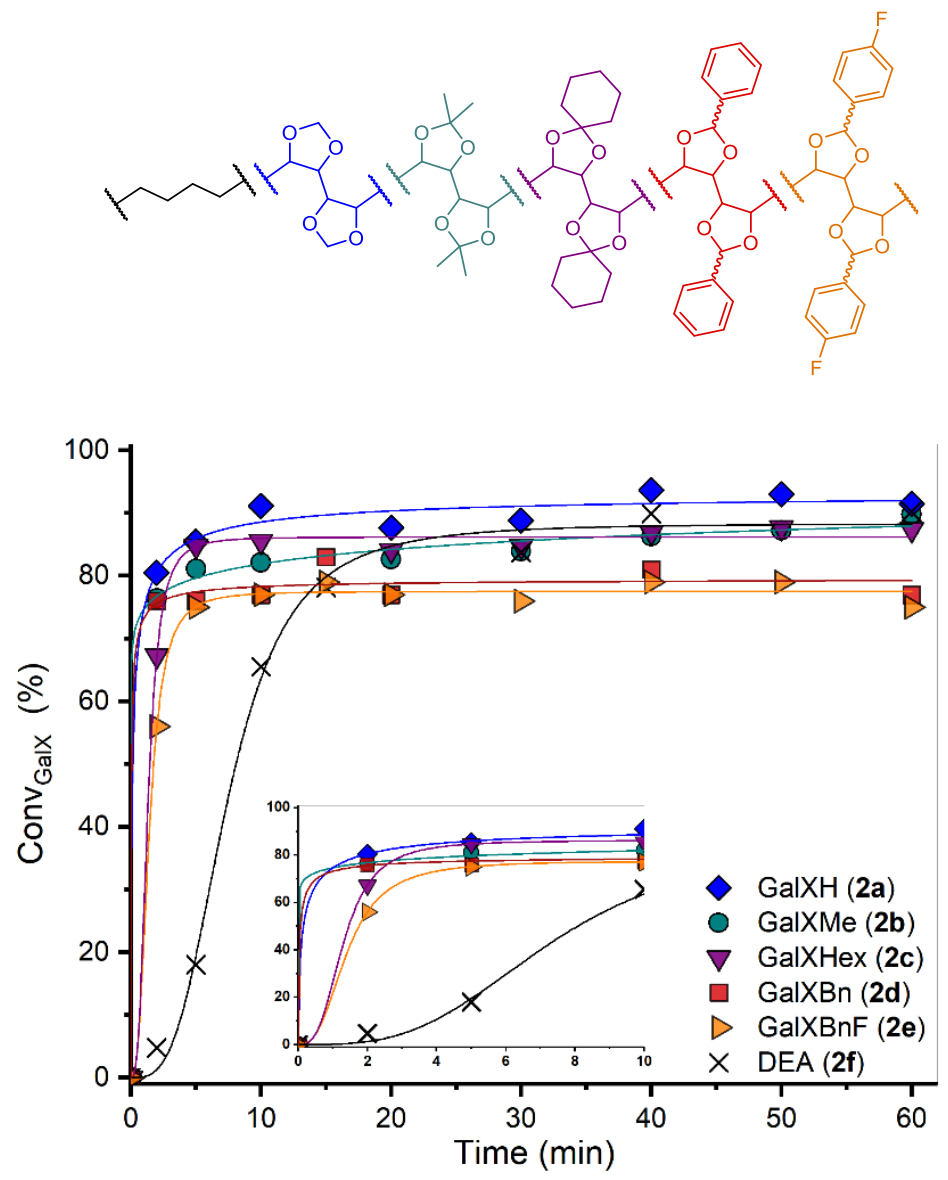

Figure 3-5 The conversion of GalX-diethyl ester groups (Conv GalX Appendix A) as a function of time for the reaction with 1,6-hexamethylenediamine at $220^{\circ} \mathrm{C}$.

The reactivity difference between GalXs with different acetal structure seems to be less pronounced than between DEA and those acetals. After 2 minutes of the reaction, the reaction of GalXH with HMDA achieves the highest consumption of the GalX monomer with $80 \%$ of reacted ethyl ester functional groups. This observation is in correspondence with the earlier conclusion from Chapter $2^{[20]}$ that GalXH is more reactive towards diamines than GalXMe. The polymerization of the aromatic acetal monomers (2d and $\mathbf{2 e}$ ) advances slower, which is also reflected in lower molecular masses of the prepared polymers (Table 3-1).

So far it is difficult to distinguish whether this difference which is also manifested in decreased polymer molecular weights is due to differences in the reactivity of the diethylesters itself, sterical hindrance or originates from the occurrence of simultaneous side reactions leading to stoichiometric imbalance. In order to investigate this further, DFT calculations have been designed to evaluate the activation of the carboxylic ester group by 
the different $\alpha$ substituents. Optimization and frequency calculations were conducted on dimethyl esters of protected and not protected galactarate or adipic acid (DMA). In a first step one molecule of methylamine reacts with one ester group and forms TS1. Subsequently this results in products of monoamidation and the release of 1 molecule of methanol (P1) (see Figure 3-6). In the second step another equivalent of the methylamine reacts with the second ester group resulting in diamide and another molecule of methanol is released (P2). Dimethyl esters instead of the diethyl esters and monoamine were chosen in order to simplify the model and to reduce the amount of atoms in the input files of the calculation.

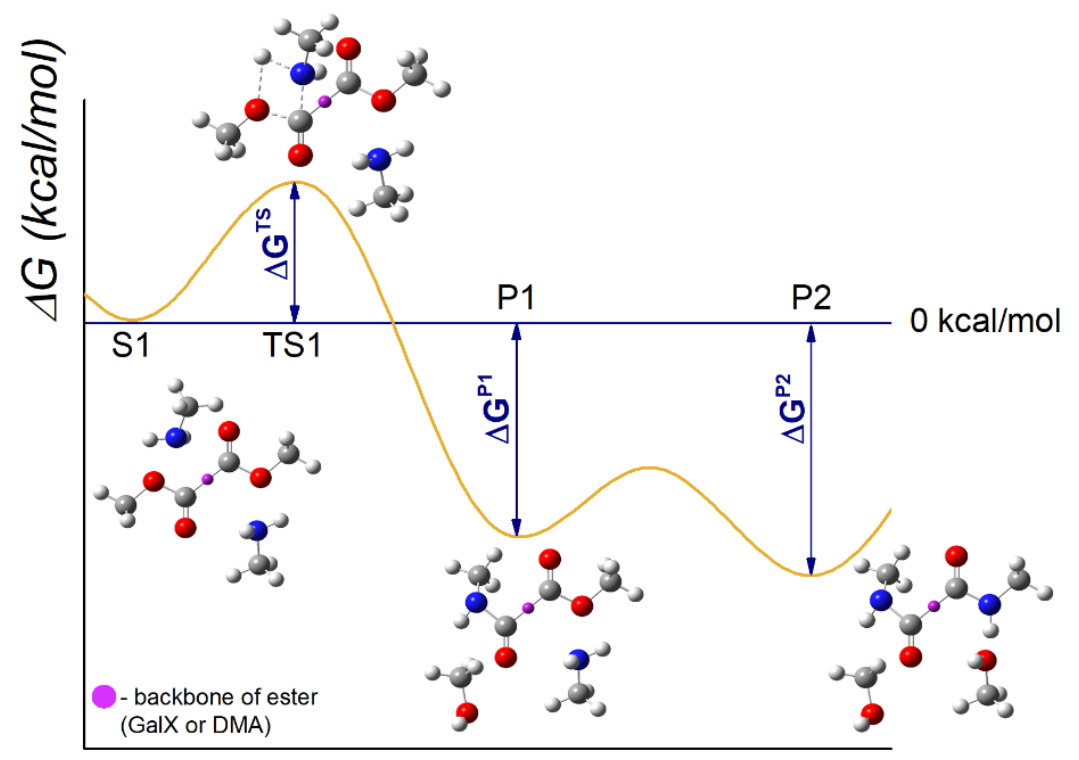

Reaction coordinates

Figure 3-6 Example of the energy profile and simplified structures of reactants i.e. dimethyl ester, methylamines methanol molecules. $\bullet$ - carbon, $\bullet-$ oxygen, $\bullet$ - nitrogen, $\bullet$-hydrogen, $\bullet$ backbone of GalX or dimethyl adipate (DMA).

The energetical output of the reaction is, in this case, expressed by the difference of the Gibbs free energy between substrate and transition state $\left(\Delta \mathrm{G}^{\mathrm{TS}}\right)$ or substrate and products $\left(\Delta \mathrm{G}^{\mathrm{P} 1}\right.$, $\left.\Delta \mathrm{G}^{\mathrm{P} 2}\right)$.

$$
G=H-T S(\mathbf{1})
$$

The Gibbs free energy $(G)$ is defined by the enthalpy of the reaction $(H)$ subtracted with the value of the entropy multiplied by the temperature (T.S) (eq. 1). The $\Delta \mathrm{G}$ is strongly related to the $\Delta \mathrm{H}$ and $\Delta \mathrm{S}$ and therefore can be used as an indicator whether the reaction is spontaneous. Entropy (disorder) and enthalpy (energy) of the system are the two driving 
forces of the reaction. In principle, the entropy is always seeking a maximum and the energy is seeking a minimum. In practice, not for every system this is true. The reaction spontaneity is determined by the value of $\Delta \mathrm{G}$, which is negative for spontaneous reactions. In Table 3-2 different cases are considered. When the energy of the system is lower after the reaction $(\Delta \mathrm{H}<0)$ and the degree of the disorder increases $(\Delta \mathrm{S}>0)$ the reaction is always spontaneous. ${ }^{27}$

Table 3-2 The reaction spontaneity considered for different energetic and entropic effects.

\begin{tabular}{llll}
\hline $\boldsymbol{\Delta H}$ & $\boldsymbol{\Delta S}$ & $\boldsymbol{\Delta G}$ & Effect \\
\hline$<0$ & $>0$ & $<0$ & Always spontaneous \\
$<0$ & $<0$ & $<0$ for $|\Delta \mathrm{H}|>|\mathrm{T} \Delta \mathrm{S}|$ & Spontaneous at low temperature \\
$<0$ & $<0$ & $>0$ for $|\Delta \mathrm{H}|<|\mathrm{T} \Delta \mathrm{S}|$ & Non-spontaneous at high temperature \\
$>0$ & $>0$ & $<0$ for $|\Delta \mathrm{H}|<|\mathrm{T} \Delta \mathrm{S}|$ & Spontaneous at high temperature \\
$>0$ & $>0$ & $>0$ for $|\Delta \mathrm{H}|>|\mathrm{T} \Delta \mathrm{S}|$ & Non-spontaneous at low temperature \\
$>0$ & $<0$ & $>0$ & Always non-spontaneous \\
\hline
\end{tabular}

The transition state formed due to the attack of an amine on the acyl carbon, results in the subsequent symmetry alteration from planar $\mathrm{sp}^{2}$ hybridization to a tetrahedral intermediate with $\mathrm{sp}^{3}$ hybridization (see Figure 3-7(a)). ${ }^{28-30}$ The direction of the attack was chosen as such that the transition state is additionally stabilized by the formation of a hydrogen bond between the amide proton and the acetal oxygen (for elucidation see Chapter 6) to ensure that the architecture of the transition state is situated in an energetical minimum. This additional stabilization of the transition structure by the interaction of the amide hydrogen (H34 Figure 3-7 b) with the acetal oxygen (O9 Figure 3-7 b) is presumably very important in case of GalX and its derivatives. The literature considers two different pathways for the aminolysis of esters, namely a stepwise mechanism, which can go through zwitterionic or neutral intermediates and the concerted pathway. ${ }^{29,31,32}$ The latter one consists of one step, in which the cleavage of a $\mathrm{C}-\mathrm{O}$ bond, the formation of a $\mathrm{C}-\mathrm{N}$ bond and a hydrogen transfer occur simultaneously. The comparison of those different chemical pathways showed that they have similar energetic output; however a multistep is slightly more energetically favorable $(2 \mathrm{kcal} / \mathrm{mol}$ for the reaction of methyl formate and ammonia ${ }^{33}$ ). Besides this, the catalytic influence of the used media and additives as well as the influence of the structure of both, ester and amine on the reaction rate have been studied. ${ }^{33,34}$ In this study no solvation effect or in a matter of fact no initial protonation of the acyl oxygen nor participation of a basic catalyst was included in the calculation since the reaction should simulate the formation of the transition state in the melt with no added catalyst to investigate the activation of ester by the strongly electronegative 
atom present on the $\alpha$ carbon. After the formation of the transition state, which is an actual maximum on the reaction coordinate graph (Figure 3-6) the proposed structure can split into product or go back to substrate.

a.

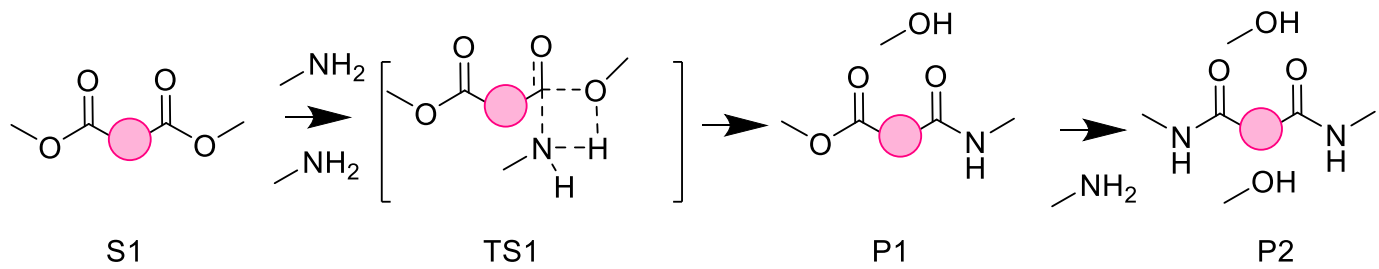

b.

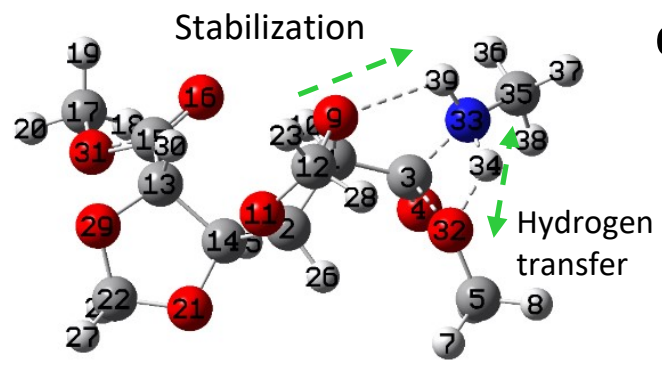

C.

$$
\Delta \mathrm{G}^{\mathrm{TS}}=40.6 \mathrm{kcal} / \mathrm{mol}
$$

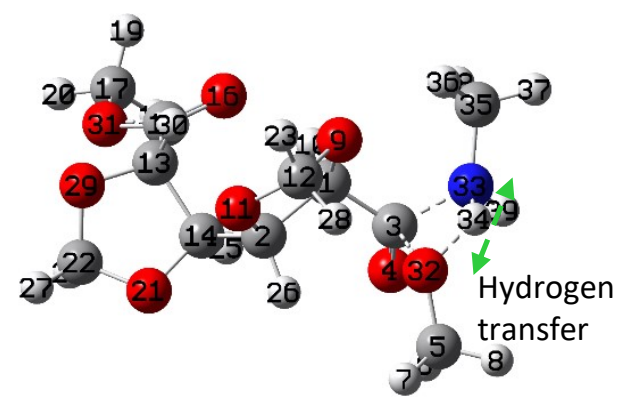

Figure 3-7 (a) The formation of a tetrahedral transition state (TS1) during the aminolysis of ester by methylamine via a concerted mechanism and (b) the structure of the transition state including stabilization of the geometry by the interaction of the amide proton with the acetal oxygen and (c) without the stabilization, together with the values of $\Delta G^{T S}$. backbone of GalX or dimethyl adipate (DMA).

Usually, the free energy balance between substrates and the transition states $\left(\Delta \mathrm{G}^{\mathrm{TS}}\right)$, called the activation energy, is higher than $0 \mathrm{kcal} / \mathrm{mol}$. In order to achieve the transition state molecules must collide with sufficient kinetic energy and proper orientation ${ }^{35}$. Only if those two conditions are met the collision is effective. The value of the activation energy determines the kinetics of the reaction in such a way that the lower it is, the faster the reaction will proceed. In Figure 3-6, the transition state is represented by the saddle point, which is a maximum point linking two minima. The lower the activation energy is, the easier the transition state can be formed. An analogous relationship can be found between substrates and products, which are represented as minima on the potential energy surface. ${ }^{36}$

The abovementioned scheme was applied to calculate the energy output of the reaction of methylamine and different Gal-derivatives including galactarate (Gal) with non-acetalized hydroxyl groups. Due to the multifunctionality of Gal the measurement of the kinetics is rather difficult since the unprotected hydroxyl groups are reacting as well. 
The prepared calculations show that the presence of an oxygen in a strategically important $\alpha$ position of the dimethyl ester of $\mathrm{Gal}(\mathrm{X})$ plays a major role in the activation. It lowers the energy barrier of the tetrahedral transition state from $48 \mathrm{kcal} / \mathrm{mol}$ for dimethyl adipate (DMA) to $32 \mathrm{kcal} / \mathrm{mol}$ for dimethyl galactarate (Figure 3-8).

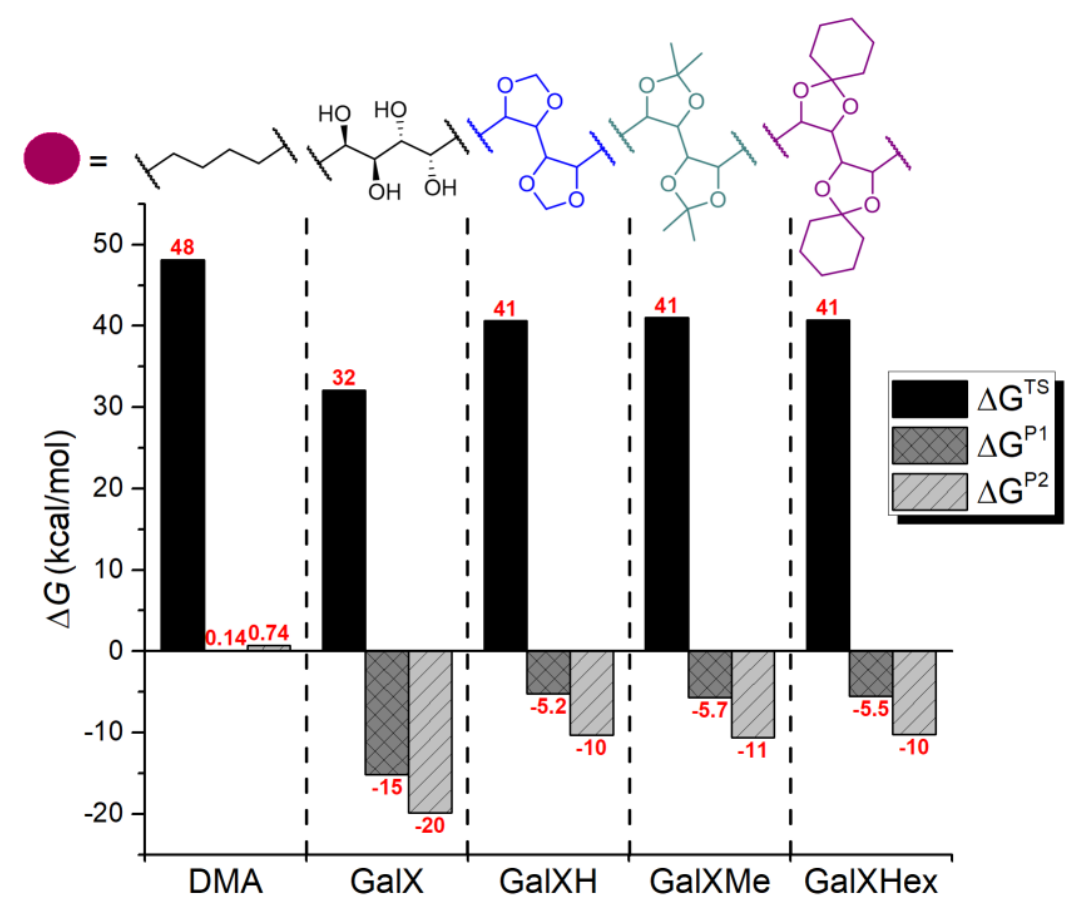

Figure 3-8 The calculated Gibbs free energy $(\Delta G)$ for different GalX dimethyl esters and dimethyl adipate (DMA) for substrates, transition states and products expressed as values relative to the substrate. $\bullet$ backbone of GalX or dimethyl adipate (DMA).

The acetalization of galactarate elevates the free energy barrier compared to non acetalized galactarate and by consequence the overall energy balance of the reaction becomes less favorable but still more favorable than for aliphatic DMA. The difference between the protected GalXs with acetals is not significant which is also manifested in the experimental kinetic data in Figure 3-5. The most pronounced role in the activation plays the presence of the oxygen substituent (hydroxyl or ether) on the $\alpha$ carbon itself.

\subsubsection{Hydrophilicity}

Polyamides are typically recognized as polymers which absorb significant amounts of water due to the presence of electron-rich sites like amide linkages. The presence of additional oxygens in the molecule of diethyl galactarate (1) and its acetals (2a-2e) can presumably contribute to increased water uptake. By means of the introduction of various acetals the hydrophilicity of the polymer can be tuned e.g. by the incorporation of bulky cycloaliphatic fragments the polymer might be more hydrophobic than typically observed for aliphatic 
polyamides. The absorbed water has a major influence on the properties of polyamides since it acts as a plasticizer, ${ }^{37}$ lowering glass transition temperatures. Moreover, water is known to induce crystallization. ${ }^{38}$

In order to investigate the water attraction by polyamides containing different types of cyclic acetal moieties, a series of polyamides (PA1-PA6) was immersed in water and the amount of absorbed water was investigated via the weight loss followed by TGA. Additionally, the influence of the absorbed water on the glass transition temperature was investigated (Figure 3-9)

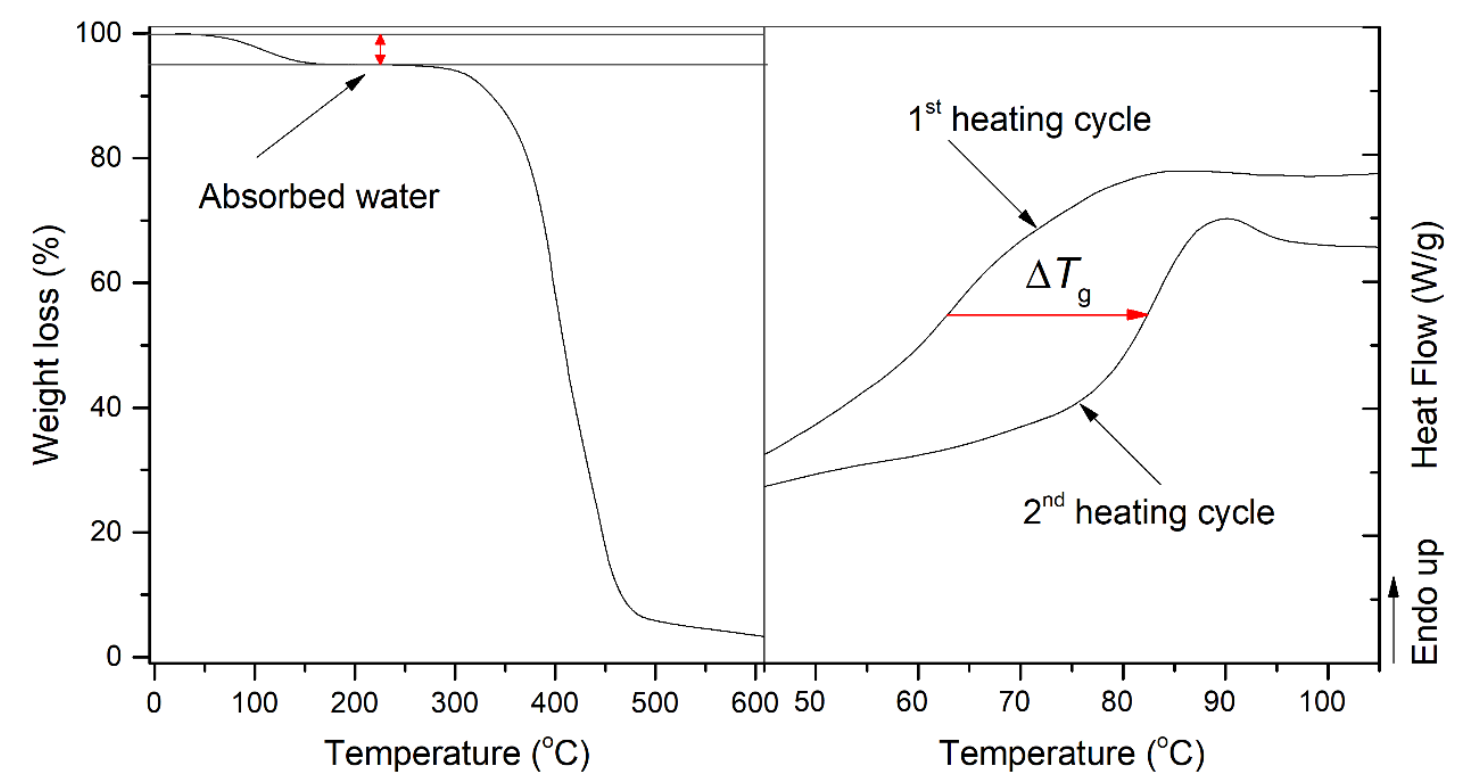

Figure 3-9 Method for the calculation of the amount of absorbed water from TGA measurement and the influence of absorbed water on the $T_{g}$ of polymers based on the DSC curves (example of polyamide PA1).

Polyamides based on GalX absorb between 1.20 (GalXBn and GalXBnF) and 4.25 $(\mathrm{GalXH}) \%$ water (Figure 3-10). There is a clear relationship between the substituents on the dioxolane ring of the acetal fragments and the water absorption as well as between the amount of absorbed water and the decrease in $T_{\mathrm{g}}$ of the material. The highest amount of water was absorbed by PA(HMDA,GalXH) PA1 (4.25\%) which exceeded the amount of water absorbed by the aliphatic PA(HMDA,DEA) PA6 reference (2.90\%). As the bulkiness and the content of hydrophobic moieties increased, the amount of absorbed water decreased below the level observed for the reference for all the other investigated GalX-based polyamides PA2-PA5. Simultaneously, the $T_{\mathrm{g}}$ of the material decreased proportionally to the amount of absorbed water resulting in the polyamides with $T_{\mathrm{g}}$ values by 26 to $8^{\circ} \mathrm{C}$ lower than the initial value. 


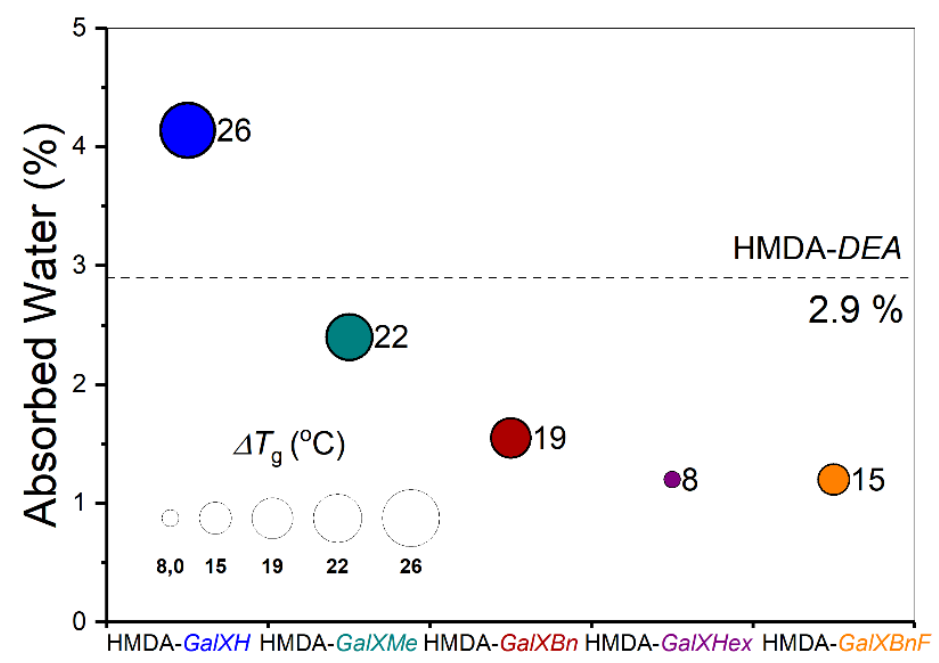

Figure 3-10 The amount of absorbed water ( $Y$-axis) and the $\Delta T_{g}$ value representing the difference in $T_{g}$ between dry polymer and polymer after immersion in water (radius of the circle and the label value) for polyamides PA1-PA5.

Besides water absorption, analysis of the obtained TGA curves revealed different stability and decomposition patterns for GalX polyamides in comparison to reference polyamide PA(HMDA-DEA). Polyamides with GalX (PA1-PA5) are considerably less stable than the reference polyamide (PA6) (Table 3-1 and Figure 3-10). Polyamides PA1-PA5 lose 10\% of their weight $60-26{ }^{\circ} \mathrm{C}$ lower than PA6 (the weight loss resulting from water absorption is already extracted from this value) and their decomposition consists of a couple of stages with different decomposition rates. Presumably, the multistage decomposition pattern of GalX polymers is caused by thermal decomposition of the acetal moieties. The stability of the aromatic moieties can be tuned by variation of the substituents on the aromatic ring. Replacement of the hydrogen in para position by fluor caused significant increase in thermal stability of PA5 (with GalXBnF) compared to PA4 (with GalXBn), resulting in $T_{\max }$ as well as $T_{10 \%}$ of polymer PA5 being $20-30{ }^{\circ} \mathrm{C}$ higher than for PA4. 


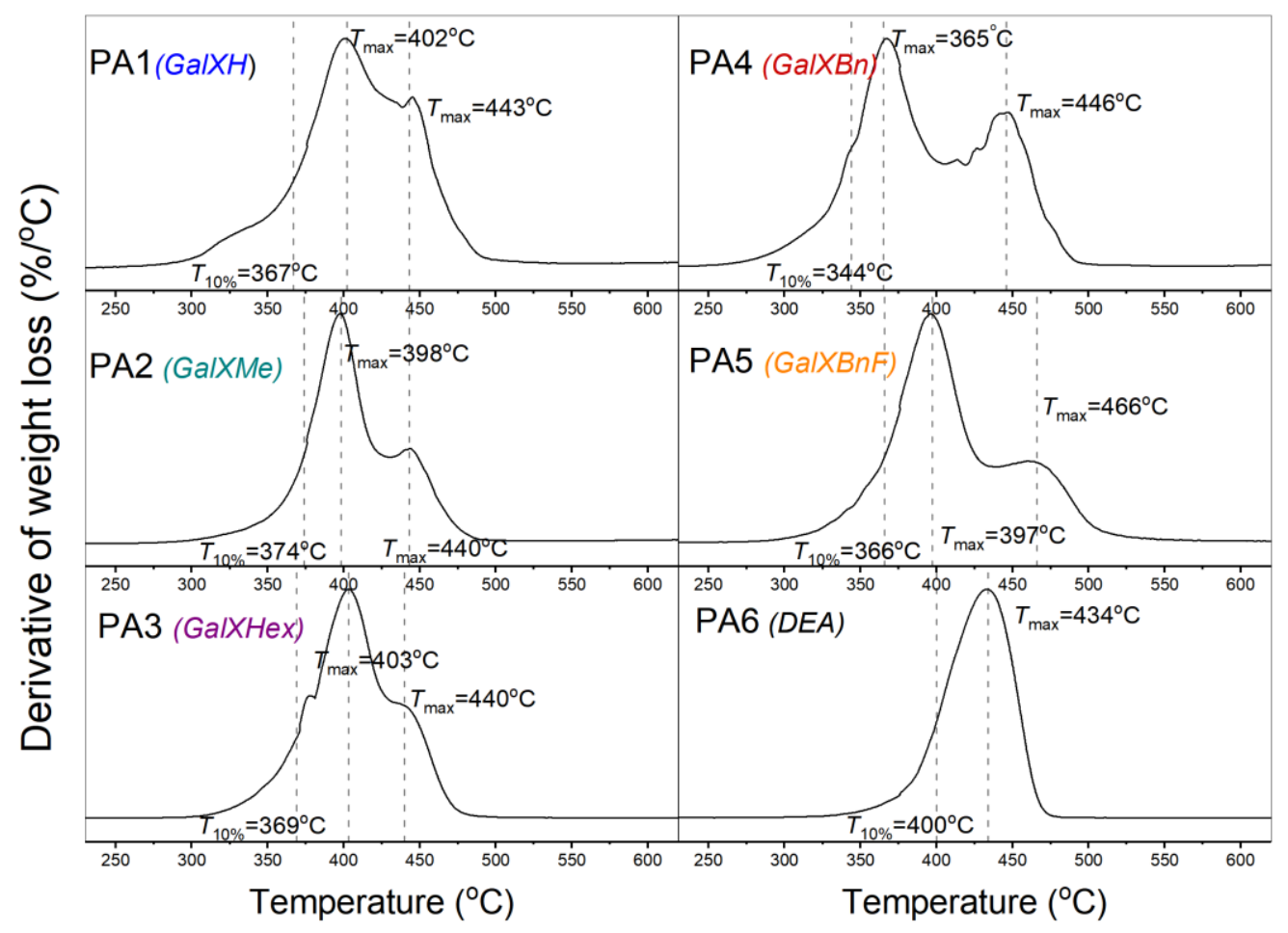

Figure 3-11 TGA curves of the prepared polyamides PA1-PA6 based on HMDA as diamine presented as a temperature derivative of weight loss.

\subsection{Conclusions}

The melt polymerization of different acetals of diethyl galactarate with 1,6 hexamethylene diamine is a challenge, mostly due to the limited thermal stability of those monomers, which can be overcome by reducing the reaction temperature compared to normal polyamide synthesis due to increased activity of the reactive ester groups in GalX. The reactivity of the GalX diethyl ester group is highly influenced by the substituent on the $\alpha$ and $\beta$ carbon of the diester meaning that the reactivity of GalX diester groups is increased compared to aliphatic diesters and therefore can successfully undergo reaction with amines within a reasonable time frame.

The polymerization of different GalX derivatives, amongst which completely new monomers like GalX with a cyclohexylidene and a fluorobenzylidene protection, was used to tune the properties of partially biobased polyamides. Not only the glass transition temperature and the thermal stability of the polyamides can be tuned in this way, but also the hydrophilicity. The hydrophilicity (water absorption) of the electron-rich acetal fragments and amide connections of the polyamides can be countered by the use of acetals with bulky hydrophobic fragments. 
The elucidation of the structure of the GalX derived polyamides by Maldi-ToF proves that the different GalX derivatives were built in into the polyamide, although some occurring side reactions lead to different types of end group functionalities.

\subsection{Material and methods}

\subsubsection{Materials}

Diethyl 2,3:4,5-di- $O$-isopropylidene-galactarate $>99 \%$, diethyl 2,3:4,5-di- $O$-methylenegalactarate $>99 \%$ and diethyl galactarate $>99 \%$ were supplied by Royal Cosun. 1,6hexamethylenediamine (HMDA) 98\%, 4-fluorobenzaldehyde $>98 \%$, benzaldehyde dimethyl acetal $>98 \%$, cyclohexanone dimethyl ketal $>98 \%$, diethyl adipate (DEA) $>98 \%$, , acetone$d_{6}$, Irganox 1330, dithranol and NaTFA were purchased from Sigma-Aldrich and used as supplied. 1,1,1,3,3,3-hexafluoro-2-propanol (HFIP), p-toluene sulfonic acid (p-TSA) >99\% and molecular sieves $4 \AA$ were purchased from Acros Organics. $\mathrm{CDCl}_{3}$ and DMSO- $d_{6}$ was purchased from Cambrige Isotope. Toluene and ethanol were purchased from Biosolve.

\subsubsection{Methods}

The procedure for the acetalization of diethyl galactarate (1) was adapted based on existing literature reports. ${ }^{15,18,39}$ NMR spectra of the prepared monomers and polyamides can be found in the Appendix B.

Diethyl 2,3:4,5-di-O-cyclohexylidene-galactarate (2c). Diethylgalactarate (2.71 g, $10 \mathrm{mmol})$, cyclohexanone dimethyl ketal $(3.6 \mathrm{~g}, 25 \mathrm{mmol})$ and p-toluene sulfonic acid (p-TSA, $40 \mathrm{mg}$, $0.2 \mathrm{mmol}$ ) were dissolved in 50-75 $\mathrm{mL}$ toluene and heated to reflux for 13 hours in a soxhlet extractor charged with $4 \AA$ molecular sieves. Sodium bicarbonate was added to a solution for neutralization. After filtration of the inorganic salts, the solvent was removed under reduced pressure and the product was recrystallized from ethanol resulting in a yellow solid mixture consisting of methyl and ethyl esters of 2.3:4,5-di- $O$-cyclohexylidene-galactarate (molar ratio methyl ester to ethyl ester 0.42:1). Yield after drying under vacuum at $50{ }^{\circ} \mathrm{C}: 2.21 \mathrm{~g}, 51.0 \%$. Melting point: $73-76^{\circ} \mathrm{C}$

${ }^{l} H-N M R(300 \mathrm{MHz}$, acetone-d6, $\delta): 4.57$ (s, 2H, CH), 4.42 (s, 2H, CH), 4.19 (q, 4H, O-CH $\left.2^{-}\right)$, 3,75 (s, 6H, O-CH3), $1.64\left(\mathrm{~s}, 16 \mathrm{H}, \mathrm{CH}_{2}\right), 1.40\left(\mathrm{~s}, 4 \mathrm{H}, \mathrm{CH}_{2}\right), 1.27\left(\mathrm{t}, 6 \mathrm{H}, \mathrm{O}-\mathrm{CH}_{3}\right)$

${ }^{13} \mathrm{C}-\mathrm{NMR}\left(75 \mathrm{MHz}\right.$, acetone- $\left.d_{6}, \delta\right): 170.70(\mathrm{C}=\mathrm{O}), 112.51(\mathrm{O}-\mathrm{C}-\mathrm{O}), 79.26(\mathrm{CH}-\mathrm{O}), 76.02$ $(\mathrm{CH}-\mathrm{O}), 60.95\left(\mathrm{CH}_{2}-\mathrm{O}\right), 51.65\left(\mathrm{CH}_{3}-\mathrm{O}\right), 36.64\left(\mathrm{CH}_{2}\right), 35.64\left(\mathrm{CH}_{2}\right), 23.77\left(\mathrm{CH}_{2}\right), 23.54\left(\mathrm{CH}_{2}\right)$, $13.47\left(\mathrm{CH}_{3}-\mathrm{C}\right)$ 
$L C-M S$ (ESI) $m / z$ : $[\mathrm{M}+1]$ calcd for $\mathrm{C}_{22} \mathrm{H}_{235} \mathrm{O}_{8}, 427.2332$ (diethyl ester), $\mathrm{C}_{21} \mathrm{H}_{33} \mathrm{O}_{8}, 413.2175$ (mixed esters), $\mathrm{C}_{20} \mathrm{H}_{31} \mathrm{O}_{8}, 399.2019$ (dimethyl ester); found: 427 (diethyl ester), 413 (mixed esters), 399 (dimethyl ester).

Diethyl 2,3:4,5-di-O-benzylidene-galactarate (2d). Diethylgalactarate (2.79 g, $10.5 \mathrm{mmol})$, benzaldehyde dimethyl acetal $(3.22 \mathrm{~g}, 22.3 \mathrm{mmol})$ and p-toluene sulfonic acid (p-TSA, 40 $\mathrm{mg}, 0.2 \mathrm{mmol}$ ) were dissolved in $50-75 \mathrm{~mL}$ toluene and heated to reflux for 13 hours in a soxhlet extractor charged with $4 \AA$ molecular sieves. The mixture was filtered and triethylamine $(0.21 \mathrm{mg}, 0.21 \mathrm{mmol})$ was added for neutralization. Upon cooling diester of 2,3:4,5-di-O-benzylidene-galactarate precipitated in the form of white crystals. The product was filtered and recrystallized from toluene resulting in a mixture of methyl and ethyl esters of 2,3:4,5-di- $O$-benzylidene-galactarate (molar ratio methyl ester to ethyl ester $0.32: 1$ ). Yield after drying under vacuum at $50{ }^{\circ} \mathrm{C}: 3.50 \mathrm{~g}, 75.5 \%$.

Melting point: $130-132{ }^{\circ} \mathrm{C}$

${ }^{l} H-N M R\left(300 \mathrm{MHz}, \mathrm{DMSO}_{6}, \delta\right): 7.61$ (s, 4H, Ar H), 7.44 (s, 6H, Ar H), 6.196 .08 (s, 2H, O-CH-O), 4.86 (s, 2H), 4.94-4.45 (m, 4H, CH), 3.79 (m, 4H, O- $\left.\mathrm{CH}_{2}-\right), 3.793 .743 .64$ (s, 3H, $\left.\mathrm{O}-\mathrm{CH}_{3}\right), 1.29\left(\mathrm{~m}, 3 \mathrm{H},-\mathrm{CH}_{3}\right)$

${ }^{13} \mathrm{C}-\mathrm{NMR}\left(75 \mathrm{MHz}, \mathrm{CDCl}_{3}, \delta\right): 170.52(\mathrm{C}=\mathrm{O}), 135.75(\mathrm{C} \mathrm{Ar}), 129.86(\mathrm{CH} \mathrm{Ar}), 128.40(\mathrm{CH}$

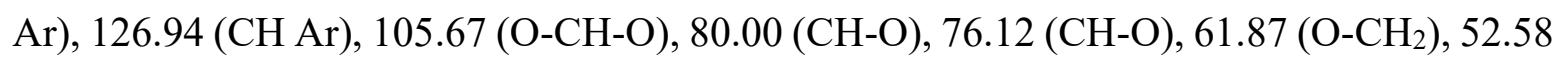
$\left(\mathrm{O}-\mathrm{CH}_{3}\right), 14.15\left(\mathrm{CH}_{3}\right)$

$L C-M S$ (ESI) $m / z$ : [M+1] calcd for $\mathrm{C}_{24} \mathrm{H}_{27} \mathrm{O}_{8}, 443,1700$ (diethyl ester), $\mathrm{C}_{23} \mathrm{H}_{25} \mathrm{O}_{8}, 429,1544$ (mixed esters), $\mathrm{C}_{22} \mathrm{H}_{23} \mathrm{O}_{8}, 415,1393$ (dimethyl ester); found: 443 (diethyl ester), 429 (mixed esters), 414 (dimethyl ester).

Diethyl 2,3:4,5-di-O-4-fluorobenzylidene-galactarate (2e). Diethylgalactarate (9.20 g, 34.0 mmol), 4-fluorobenzaldehyde (12.9 g, $100 \mathrm{mmol})$ and p-toluene sulfonic acid (p-TSA, 220 $\mathrm{mg}, 1.10 \mathrm{mmol}$ ) were dissolved in $125 \mathrm{~mL}$ toluene and heated to reflux for 24 hours in a soxhlet extractor charged with $4 \AA$ molecular sieves. The mixture was filtered and triethylamine $(121 \mathrm{mg}, 1.21 \mathrm{mmol})$ was added for neutralization. Upon cooling at $-20{ }^{\circ} \mathrm{C}$, the diester of 2,3:4,5-di-O-4-fluorobenzylidene-galactarate precipitated in the form of white crystals. The product was filtered and recrystallized from ethanol resulting in $1.9 \mathrm{~g}$ monomer after drying under vacuum at $50{ }^{\circ} \mathrm{C}(4.0 \mathrm{mmol}$, yield $11.5 \%)$.

Melting point: $154-156^{\circ} \mathrm{C}$

${ }^{1} \mathrm{H}-\mathrm{NMR}\left(300 \mathrm{MHz}, \mathrm{CDCl}_{3}, \delta\right.$ in ppm): 7.54 (m, 4H, Ar H), 7.08 (m, 6H, Ar H), 6.196 .17 6.08 (s, 2H, O-CH-O), 4.94-4.45 (m, 4H, CH), 4.27 (m, 4H, O-CH $\left.2^{-}\right), 1.31$ (m, 3H, - $\left.\mathrm{CH}_{3}\right)$ 
${ }^{13} \mathrm{C}-\mathrm{NMR}\left(75 \mathrm{MHz}, \mathrm{CDCl}_{3}\right.$, $\delta$ in ppm): $170.39170 .22169 .97169 .46(\mathrm{C}=\mathrm{O}), 165.33162 .04$ (F-C Ar), 131.62 129.01 115.58 (C Ar), 105.78105 .42105 .10105 .01 (O-CH-O), 80.4175 .93 $79.7479 .2976 .2675 .9275 .8375 .52(\mathrm{CH}-\mathrm{O}), 61.9861 .90\left(\mathrm{O}-\mathrm{CH}_{2}\right), 14.2314 .1614 .0714 .12$ $\left(\mathrm{CH}_{3}\right)$

LC-MS (ESI) m/z: [M+1] calcd for $\mathrm{C}_{24} \mathrm{H}_{24} \mathrm{~F}_{2} \mathrm{O}_{8}, 479.44 \mathrm{~g} / \mathrm{mol}$; found: $479 \mathrm{~g} / \mathrm{mol}$.

A general procedure for the polycondensation of GalX derivatives with adipic acid diethyl ester is presented in Chapter 2.

\subsubsection{Characterization}

The thermal stability and water uptake was determined by TGA. ${ }^{1} H N M R$ and ${ }^{13} C N M R$, molecular weight determined by GPC, Maldi-ToF spectra and melting temperatures were determined/recorded as described in the experimental section in Chapter 2.

Water uptake was measured after the immersion of a polymer sample in water for 72 hours and subsequent drying for 24 hours in air. The weight loss observed around $100{ }^{\circ} \mathrm{C}$ on the TGA curve was interpreted as the amount of absorbed water (\%).

Liquid chromatography - mass spectrometry analysis was performed on a Shimadzu system equipped with a Shimadzu Nexera HPLC solvent delivery system (LC-30AD) with photodiode array detector (PDA/SPD-M20A), evaporative light scattering detector (ELSD-LTII) and a single quadrupole mass spectrometer (LCMS 2020). The MS was attributed in a dual ionization source consisting of both electron spray ionization (ESI) and atmospheric pressure chemical ionization (APCI). The separation was performed on Waters X-Select CSH C18 columns $(3.5 \mu \mathrm{m}, 3 \mathrm{~mm} \times 75 \mathrm{~mm})$ with a solvent gradient of $5 \%$ acetonitrile in water for 2 minutes followed by a gradient to $95 \%$ acetonitrile in water over $6 \mathrm{~min}$ and flushing of the column at $95 \%$ for 2 min. Both solvents were modified with $0.1 \%$ formic acid.

The DSC thermograms were recorded using differential scanning calorimetry (DSC) on a Netzsch Polyma 2014 DSC. DSC data was obtained from about $5 \mathrm{mg}$ of polymer at heating/cooling rates of $10{ }^{\circ} \mathrm{C} \mathrm{min}^{-1}$ under a nitrogen flow of $20 \mathrm{~mL} \mathrm{~min}^{-1}$. Indium, zinc, tin and bismuth were used as standards for temperature and enthalpy calibration. DSC heating and cooling cycles were performed from 0 to $250{ }^{\circ} \mathrm{C}$. The melting temperatures (top value) and the enthalpy values reported correspond to the second heating cycle.

Potential energy surfaces (PES) were calculated using a density functional theory (DFT) method with diffusion functions on heavy atoms and polarization functions on hydrogen using the B3-LYP/6-31G $(\mathrm{d}, \mathrm{p})$ basis set with the Gaussian 09 and GaussView 05 software 
Chapter 3

package. ${ }^{40}$ The substrates and products were optimized to the minimum structure with 0 negative frequency. Transition states were optimized to the structure, which is maximum in one direction and minimum in all others (TS Berry) with exactly 1 negative frequency. To confirm the location of a correct transition state structure, intrinsic reaction coordinates analysis (IRC) was conducted. The calculations were conducted at $298 \mathrm{~K}$ under $1 \mathrm{Atm}$ in gas phase on Intel ${ }^{\circledR}$ Xeon ${ }^{\circledR}$ workstation equipped with $\mathrm{CPU}$ E5-2650 v4, $2.2 \mathrm{GHz}$ and $32 \mathrm{~GB}$ ram memory. 


\section{REFERENCES}

1. C. M. Kshirsagar and R. Anand, Appl. Mech. Mater., 2014, 592-594, 1881-1885.

2. K. Srirangan, L. Akawib, M. Moo-Younga and C. P. Choua, Appl. Energy, 2012, 100, 172186.

3. D. E. Kiely, A. Vishwanathan, B. P. Jarman and M. Manley-Harris, J. Carbohydr. Chem., 2009, 28, 348-368.

4. B. P. Jarman, D. E. Kiely, M. Manley-Harris and B. K. Nicholson, J. Carbohydr. Chem., 2009, 28, 107-123.

5. $\quad$ S. Munoz-Guerra, High Perform. Polym., 2012, 24, 9-23.

6. C. Lavilla, A. Alla, A. Martínez de Ilarduya, E. Benito, M. G. García-Martín, J. A. Galbis and S. Muñoz-Guerra, J. Polym. Sci., Part A: Polym. Chem., 2012, 50, 3393-3406.

7. C. Lavilla and S. Muñoz-Guerra, Polym. Degrad. Stab., 2012, 97, 1762-1771.

8. C. Lavilla, A. Alla, A. Martínez de Ilarduya, E. Benito, M. G. García-Martín, J. A. Galbis and S. Muñoz-Guerra, J. Polym. Sci., Part A: Polym. Chem., 2012, 50, 1591-1604.

9. C. Lavilla, E. Gubbels, A. Martínez de Ilarduya, B. A. J. Noordover, C. E. Koning and S. Muñoz-Guerra, Macromolecules, 2013, 46, 4335-4345.

10. C. Lavilla and S. Muñoz-Guerra, Green Chem., 2013, 15, 144-151.

11. V. Froidevaux, C. Negrell, S. Caillol, J. P. Pascault and B. Boutevin, Chem. Rev., 2016, 116, 14181-14224.

12. M. Winnacker and B. Rieger, Macromol. Rapid Commun., 2016, 37, 1391-1413.

13. C. Rosu, I. I. Negulescu, R. Cueto, R. Laine and W. H. Daly, J. Macromol. Sci., Part A Pure Appl. Chem., 2013, 50, 940-952.

14. M. Acemoglu, S. Bantle, T. Mindt and F. Nimmerfall, Macromolecules, 1995, 28, 3030-3037.

15. C. Hahn, H. Keul and M. Möller, Polym. Int., 2012, 61, 1048-1060.

16. B. Begines, F. Zamora, E. Benito, M. de Gracia García-Martín and J. A. Galbis, J. Polym. Sci., Part A: Polym. Chem., 2012, 50, 4638-4646.

17. M. Metzke, N. O'Connor, S. Maiti, E. Nelson and Z. Gua, Angew. Chem. Int. Ed., 2005, 44, 6529-6533.

18. T. P. Bird, W. A. P. Black, E. T. Dewar and H. W. T. Rintoul, J. Chem. Soc., 1964, 0, $4512-$ 4521.

19. T. P. Bird, W. A. P. Black, E. T. Dewar and J. B. Hare, J. Chem. Soc., 1963, 0, 3389-3391.

20. A. A. Wróblewska, K. V. Bernaerts and S. M. A. De Wildeman, Polymer, 2017, 124, 252262.

21. M. E. Rogers and T. E. Long, Synthetic methods in step-growth polymers., A John Wiley \& Sons, inc., Hoboken, New Jersey, 2003.

22. Polymer Handbook, John Wiley \& Sons, Inc., New York - Chichester - Brisbane - Toronto, 1975.

23. N. Ogata, K. Sanui, K. Hosoda and H. Nakamura, J. Polym. Sci., Polym. Chem. Ed., 1976, 14, 783-792.

24. N. Ogata, K. Sanui, K. Hosoda and H. Nakamura, J. Polym. Sci., Polym. Chem. Ed., 1977, 15, 1523-1526.

25. E. Hellmann, J. Malluche and G. P. Hellmann, Polym. Eng. Sci., 2007, 47, 1600-1609.

26. J. E. Flannigan and G. A. Mortimer, J. Polym. Sci., Part A: Polym. Chem., 1978, 16, 1221 1228.

27. Peters; A.P.H., Concise chemical thermodynamics, CRC Press, Boca Raton, 2010.

28. D. D. Sung, I. S. Koo, K. Yang and I. Lee, Chem. Phys. Lett., 2006, 426, 280-284.

29. M. J. Gresser and W. P. Jencks, J. Am. Chem. Soc., 1977, 99, 6963-6970.

30. I.-H. Um, S.-E. Lee and H.-J. Kwon, J. Org. Chem., 2002, 67, 8999-9005.

31. J. F. Bunnett and G. T. Davis, J. Am. Chem. Soc., 1960, 82, 665-674.

32. A. C. Satterthwait and W. P. Jencks, J. Am. Chem. Soc., 1974, 96, 7018-7031.

33. S. Ilieva, B. Galabov, D. G. Musaev, K. Morokuma and H. F. Schaefer, J. Org. Chem., 2003, 68, 1496-1502.

34. W. Yang and D. G. Drueckhammer, Org. Lett., 2000, 2, 4133-4136. 
35. lumenlearning, https://courses.lumenlearning.com/boundless-chemistry/chapter/activationenergy-and-temperature-dependence/, (accessed 25 April, 2018).

36. J. B. Foresman and A. Frisch, Exploring Chemistry with Electronic Structure Methods, Gaussian, Inc., Wallingford, CT USA, 3rd edn., 2015.

37. N. Reuvers, H. Huinink and O. Adan, Macromol. Rapid Commun., 2013, 34, 949-953.

38. C. Vergelati, A. Imberty and S. Perez, Macromolecules, 1993, 26, 4420-4425.

39. H. Zinner, A. Koine and H. Nimz, Chem. Ber., 1960, 93, 2791-2803.

40. Gaussian 09, Revision E.01, M. J. Frisch, G. W. Trucks, H. B. Schlegel, G. E. Scuseria, M. A. Robb, J. R. Cheeseman, G. Scalmani, V. Barone, B. Mennucci, G. A. Petersson, H. Nakatsuji, M. Caricato, X. Li, H. P. Hratchian, A. F. Izmaylov, J. Bloino, G. Zheng, J. L. Sonnenberg, M. Hada, M. Ehara, K. Toyota, R. Fukuda, J. Hasegawa, M. Ishida, T. Nakajima, Y. Honda, O. Kitao, H. Nakai, T. Vreven, J. A. Montgomery, J. E. Peralta, F. Ogliaro, M. Bearpark, J. J. Heyd, E. Brothers, K. N. Kudin, V. N. Staroverov, T. Keith, R. Kobayashi, J. Normand, K. Raghavachari, A. Rendell, J. C. Burant, S. S. Iyengar, J. Tomasi, M. Cossi, N. Rega, J. M. Millam, M. Klene, J. E. Knox, J. B. Cross, V. Bakken, C. Adamo, J. Jaramillo, R. Gomperts, R. E. Stratmann, O. Yazyev, A. J. Austin, R. Cammi, C. Pomelli, J. W. Ochterski, R. L. Martin, K. Morokuma, V. G. Zakrzewski, G. A. Voth, P. Salvador, J. J. Dannenberg, S. Dapprich, A. D. Daniels, O. Farkas, J. B. Foresman, J. V. Ortiz, J. Cioslowski and D. J. Fox, Gaussian, Inc., Wallingford CT, 2013 


\section{Chapter 4 SYNTHESIS OF GALX COPOLYAMIDES}

Abstract

This chapter focuses on the preparation of copolyamides containing biacetalized 2,3:4,5-diO-isopropylidene-galactaratic acid (GalXMe) and 2,3:4,5-di-O-methylene-galactaric acid $(\mathrm{GalXH})$, in bulk by melt polycondensation of salt monomers. In order to allow the incorporation of temperature-sensitive sugar-derived building blocks into copolyamides at temperatures below the degradation temperature of the monomers and below their melting temperatures, a clever selection of salt monomers is required, such that the sugar-derived salt monomer dissolves in the other salt monomers. The polymerization is investigated by temperature dependent FT-IR and optical microscopy. The structure of the obtained copolyamides is elucidated by NMR and Maldi-ToF techniques. The positive outcome of this modified polycondensation method is dependent on the solubility of the monomers in each other and the difference between the melting temperatures of the comonomers, however does not depend on the melting temperature of used sugar-derived monomer. A variety of comonomers is screened in order to establish the underlying mechanisms of the process. 



\subsection{Introduction}

The common method for the synthesis of polyamides is the melt polymerization of polyamide salts but it cannot be directly applied for the synthesis of polyamides from galactaric acid derivatives due to their rapid degradation occurring simultaneously with melting.

The poor thermal stability is a common problem for a variety of carbohydrate-derived monomers and can be addressed by the application of different techniques like solid state polymerization (SSP) ${ }^{1,2}$ or solution polymerization. ${ }^{3-5}$ SSP is a bulk polymerization technique that involves heating of the starting material (crystalline monomer or semicrystalline prepolymer prepared by melt condensation) at temperatures between the glass transition temperature and the onset of melting. The mobility of chain ends present in the amorphous phase allows further reaction resulting in high molecular weight polymer. ${ }^{6,7}$ Reaction byproducts are removed by the application of vacuum or through convection caused by passing an inert gas. The technique can be applied on dry monomers (e.g. polyamide salt, amino acid) as well as for the postcondensation of prepolymers (generally obtained by conventional melt condensation), ${ }^{8,9}$ and for incorporation of another monomer into the polymer chain by means of transesterification for polyesters 10,11 or aminolysis/transamidation for polyamides. ${ }^{6,12}$ Executing the reaction below the melting point of the polymer is beneficial with regard to thermal stability of the monomer and the polymer. Therefore, solid state polymerization attracts much attention for the synthesis of semicrystalline (co)polyesters and/or (co)polyamides from carbohydrate-derived cyclic monomers like isosorbide $8,9,13,14$, glucaric acid, mannaric acid and galactaric acid derivatives 2, 13, 15, 16 or aromatic 2,5-furandiacarboxylic acid. ${ }^{17-19}$ The reaction rate of solid state polymerization is significantly lower compared to melt and solvent processes, which might also cause problems connected with the sublimation of monomers.

Another method for the polymerization of temperature-sensitive monomers by polycondensation is solution polymerization which can be typically performed below $100{ }^{\circ} \mathrm{C}$. ${ }^{20-24}$ Different solution polymerization approaches like acid activation via phosphorylation or interfacial polymerization from acyl chlorides have been reported for carbohydrate-derived cyclic monomers ${ }^{13,25-33}$ However, the presence of significant volumes of (toxic) solvent in the reaction environment (like N-methylpyrrolidone in case of phosphorylation ${ }^{3-5}$ ) causes that the drawbacks outweigh the benefit of the low temperature applied in those processes. 


\section{Chapter 4}

This research focuses on the preparation of copolyamides containing biacetalized galactaric acid (GalX) 2,3:4,5-di-O-isopropylidene-galactarate (GalXMe) and 2,3:4,5-di- $O$-methylenegalactarate $(\mathrm{GalXH})$ in bulk by a technique which enables salt polymerization at lower temperatures than one would normally expect based on the melting point of the starting salts. The copolymers are prepared from a mixture of GalX and non-GalX containing salts at a temperature below the $T_{\mathrm{m}}$ of the GalX salt in order to restrain the degradation of the temperature-sensitive protected galactaric acid units above the $T_{\mathrm{m}}$. Heating of the salt mixture initially gives a suspension with solid particles of GalX containing salt in molten non-GalX containing salt. Further heating results in dissolution of the solid particles and polymerization proceeds in the melt. The melt polycondensation procedure with mixed salts benefits from higher reaction rates than solid state condensation and avoids the use and regeneration of environmentally unfriendly solvents (solution polycondensation). The prepared copolyamides are characterized by ${ }^{1} \mathrm{H}$ NMR and Maldi-ToF and the polymerization process is followed under an optical microscope and with the time dependant FT-IR.

\subsection{Synthesis of copolyamides}

In order to allow copolymerization of thermally sensitive GalX salts they were mixed with a series of non-GalX containing polyamide salts, resulting in copolymers. The chosen diamines and dicarboxylic acids were varied in terms of their chemical structures. GalX-diamine salts were copolymerized with aliphatic salts with different lengths of the aliphatic chain (DDDA,SA and HMDA, salts) and aromatic/aliphatic salts (1,3-phenylenedimethanamine1,12-dodecanedioic acid salt (MXD,DA salt)). The GalX-salt and non-GalX salt were mixed together in different molar ratios and heated resulting in the copolymers presented in Figure 4-1. The content of biobased monomers of the prepared copolyamides ranges between 62 $\mathrm{wt} \%$ to $100 \mathrm{wt} \%$. It depends on which components were used during the synthesis i.e. 1,6hexamethylenediamine (from adipic acid, derived from e.g. glucose ${ }^{34}$ ), sebacic acid (extracted from castor oil or ricinoleic acid ${ }^{35}$ ), 1,12-dodecanedioic acid (from fatty acids ${ }^{36}$ ) and GalXMe (see Figure 4-1 (a)) ${ }^{37}$ can be fully bio-derived and are commonly used monomers in industrial polyamide production. 


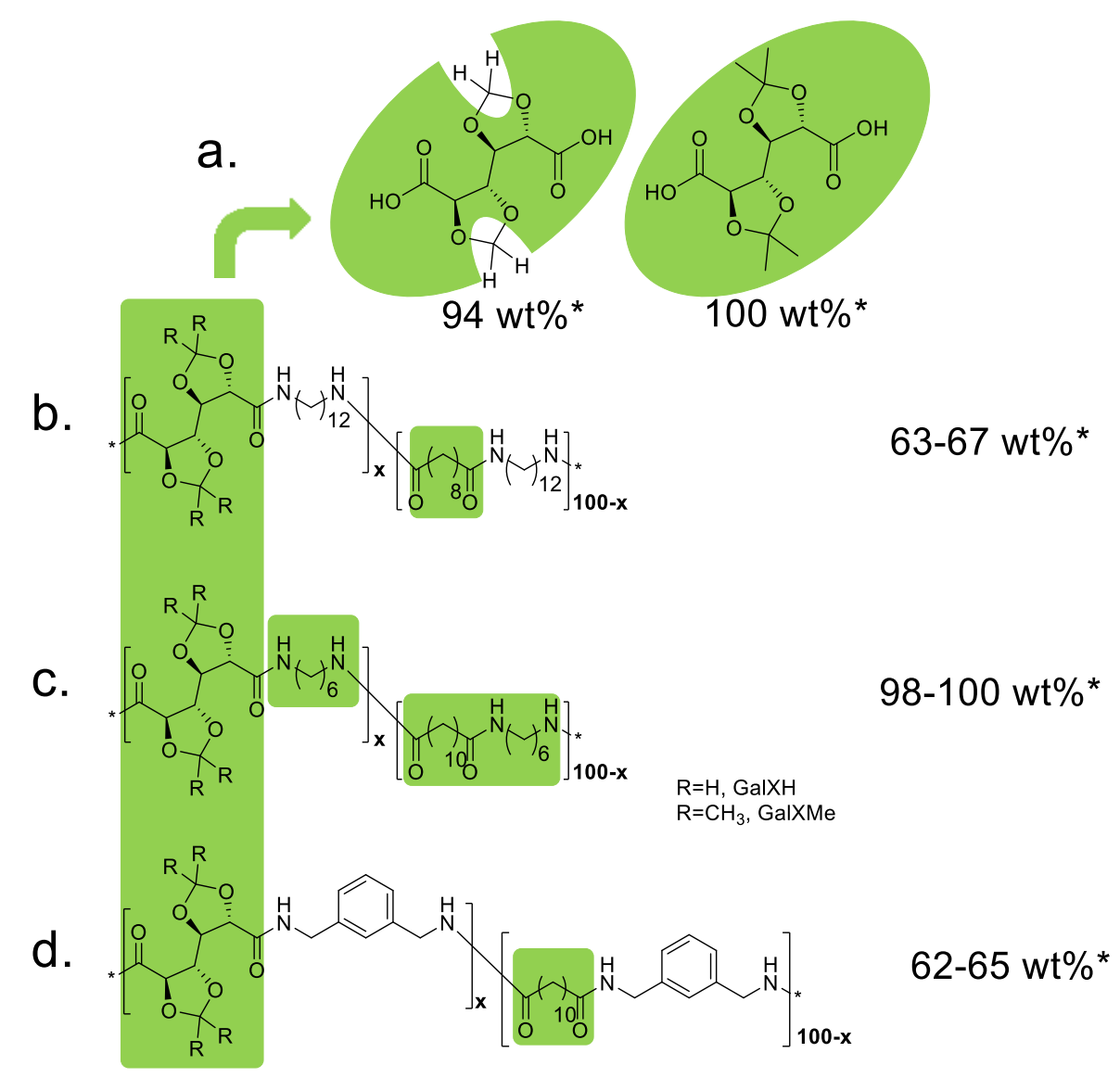

Figure 4-1 Chemical structures of (a) the GalX monomers and the prepared copolyamides thereof; (b) $P A(D D D A, G a l X R)-c o-P A(D D D A, S A), \quad(c)$ PA(HMDA,GalXR)-co-PA(HMDA,SA), (c) PA(MXD, GalXR)-co$P A(M X D, D A)$ together with the bio-based content represented by the green frames. The biobased content (wt\%) depends on the used monomers and the mol\% of each component in the copolymer.

The GalX-salt and the non-GalX salt were combined and heated till around above the melting point of the non-GalX salt (typical reaction temperatures in Figure 4-1), which is much lower (and thus a milder reaction temperature) than the melting point of the GalX salt (Figure 4-4). During the synthesis it was observed that only certain combinations of salts can be polymerized (Table 4-1).

Generally, GalXH copolyamides prepared from salts show lower dispersities than the corresponding copolyamides from GalXMe (Table 4-1). It is hypothesized that the combination of heat, released water (see Figure 4-2 (a)) and acidity (acid functional GalX monomer (see Figure 4-2 (b)) induces more deprotection of GalXMe than for GalXH. In order to support this hypothesis, a thermal stability study was conducted on four different GalX molecules: two dicarboxylic acids of GalXH and GalXMe and the corresponding two diethyl ester derivatives. A first visual observation (Figure 4-3 (e)) showed that when exposed to high temperature $\left(220^{\circ} \mathrm{C}\right.$ ), the esters of both monomers are more stable (no or slight 


\section{Chapter 4}

discoloration) than the acids (black color). This is attributed to internally catalyzed hydrolysis of the acetal, where the GalX dicarboxylic groups act as proton donors inducing acidic hydrolysis.

a.
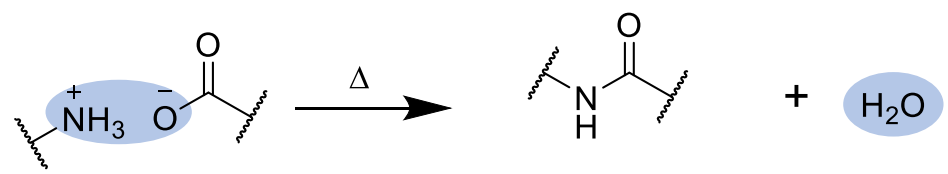

b.<smiles>CCC1OC(C)(C)OC1C(=O)O</smiles><smiles>CCC1OC(C)(C)OC1C(=O)[O-]</smiles>

\section{c.}
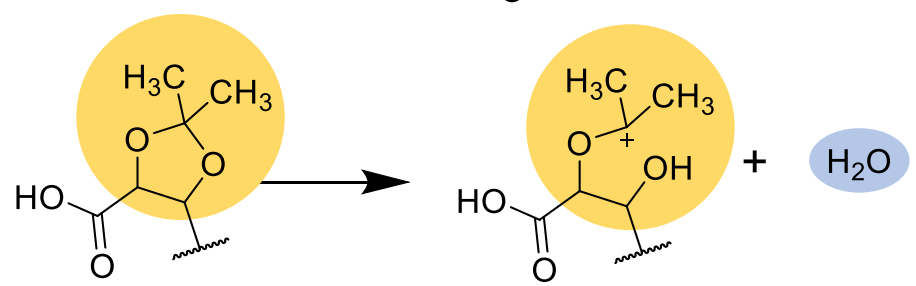

Figure 4-2 A scheme representing the internally induced hydrolysis a. the formation of water during salt polymerization $b$. generation of protons and c. the formation of a stable carbocation from GalXMe.

The formation of an intermediate tertiary carbocation favors GalXMe deprotection (see Figure 4-2 (c)) while such a stabilized carbocation cannot be formed for GalXH which is reflected in the higher stability of the respective molecules. The NMR spectra of GalXH dicarboxylic acid after exposure to elevated temperature reveal fragments related to intact GalX moieties (protons 1-6 Figure 4-3(b)), but also some side products although GalXH should be stable in acidic conditions according to literature. ${ }^{16,38-41}$ The literature reports about the acidic hydrolysis of GalX derivatives are however performed below $100{ }^{\circ} \mathrm{C}$, while that experiment uses more extreme conditions $\left(220^{\circ} \mathrm{C}\right.$ and acidity from the acid groups of GalX). When GalXMe diacid is exposed to the same conditions, almost full deprotection is observed (Figure 4-3(d)) and the predominant signal corresponds to the acetone released due to the acidic hydrolysis. This is also supported by the statement in Greene's book ${ }^{42}$ that in general the removal of acetal protective groups under acidic conditions is straightforward except for methylene protections like in GalXH. This simple experiment, which reveals the different stability of both GalX dicarboxylic acid monomers at elevated temperatures, also showed that the opposite statement is true for the GalX diethyl esters: the GalXMe diethyl ester shows no transformations like discoloration or additional peaks in the ${ }^{1} \mathrm{H}$ NMR spectrum (Figure 4-3(c) and (e)) during the heat exposure experiment, while GalXH diethyl ester is less stable as seen 
from the discoloration (Figure 4-3(e)) and the appearance of additional peaks above 8 ppm in Figure 4-3(a) (presumably aldehyde/carboxylic acid degradation products). The opposite stability of GalXH and GalXMe upon heating to $220{ }^{\circ} \mathrm{C}$ in the presence or absence of acidic end groups, can be explained by the fact that pure thermal degradation is probably dominated by radical processes ${ }^{43,44}$ while acidic degradation by cationic processes. ${ }^{38,45}$ In Chapter 2 it was demonstrated that GalXH diethyl ester gave polyamides with higher dispersities than GalXMe diethyl ester, with higher dispersities attributed to partial GalX deprotection resulting in branching/crosslinking.
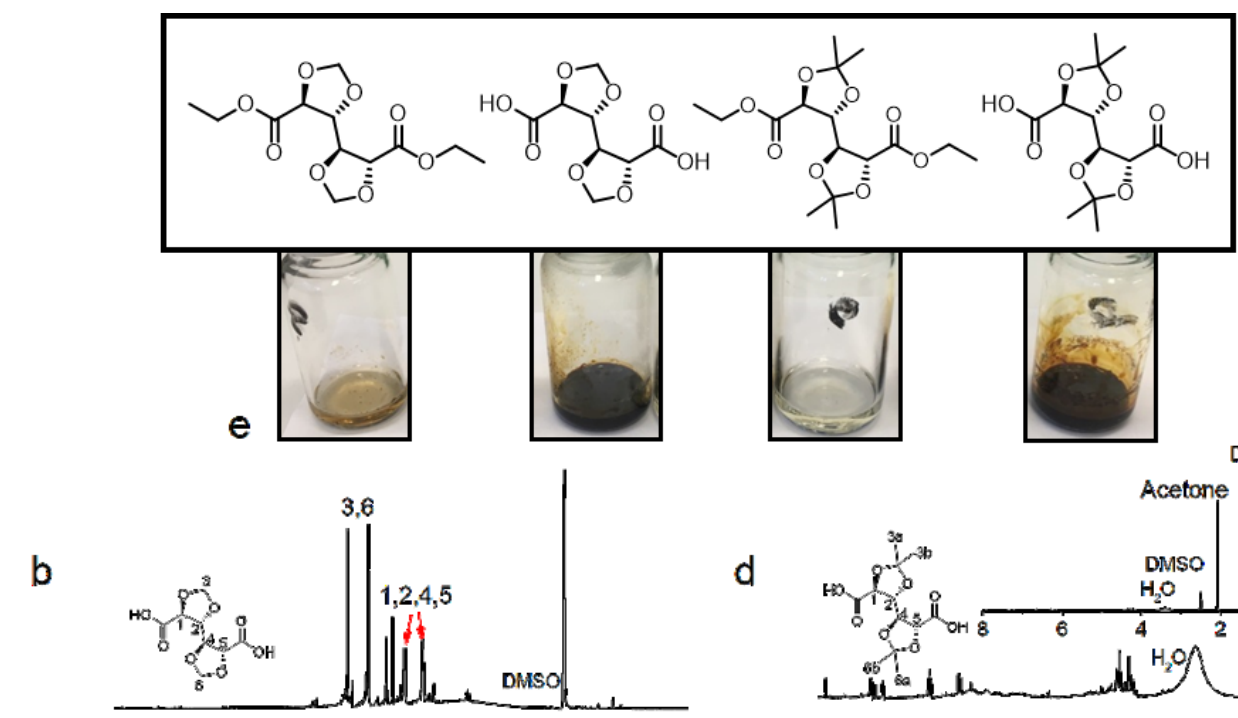

d
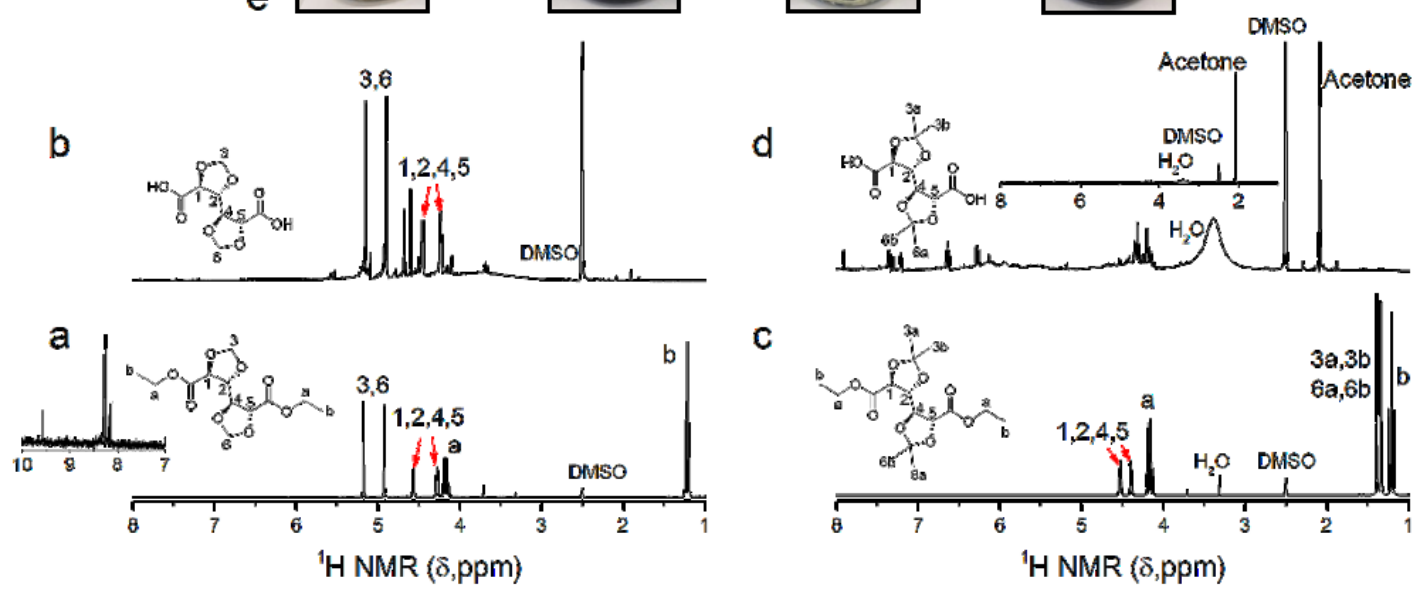

Figure 4-3 ${ }^{1} H$ NMR results of the stability test and reaction vials of four derivatives of GalX: (a) GalXH diethyl ester, (b) GalXH dicarboxylic acid, (c) GalXMe diethyl ester, (d) GalXMe dicarboxylic acid and (e) images of the compounds after the heat treatment. The test was performed in closed vials at $220^{\circ} \mathrm{C}$ under nitrogen atmosphere over a course of 5 minutes.

The higher susceptibility of GalXMe than GalXH to degradation in the presence of heat, released water and acidity is also confirmed by NMR analysis of the polyamides. The NMR spectra of polyamides containing GalXH (Appendix C) reveal the effective incorporation of GalXH monomer into the copolymer, with a reasonably good correspondence between the amount of GalX in the feed and in the final polymer (Table 4-1). The synthesis of GalXMe copolymers was significantly more challenging, with PA(DDDA,GalXMe)-coPA(DDDA,SA) being more successful (higher molecular weights) than 
PA(HMDA,GalXMe)-co-PA(HMDA,DA). When looking at the correlation between the amount of GalXMe in the feed and in the final polymer it turns out that for PA(HMDA,GalXMe)-co-PA(HMDA,DA) there is a big discrepancy between both values, explaining the lower molecular weights (stoichiometry imbalance). In fact, a significant decomposition of the GalXMe monomer is occurring in case of PA(HMDA,GalXMe)-coPA(HMDA,DA), similar as was observed during the degradation study (Figure 4-2). The higher the amount of GalXMe, the more degradation is happening and the worse the correspondence between feed/final polymer composition. The tendency of GalXMe degradation is lower in more hydrophobic media, explaining the better results (higher molecular weight, higher GalXMe content in the final polymer) for PA(DDDA,GalXMe)-coPA(DDDA,SA) compared to PA(HMDA,GalXMe)-co-PA(HMDA,DA).

Table 4-1 Composition and molecular weights of copolyamides.

\begin{tabular}{|c|c|c|c|c|c|c|c|c|c|}
\hline \multicolumn{2}{|c|}{ Composition } & \multirow{2}{*}{$\begin{array}{c}T \\
\text { reaction } \\
\left({ }^{\circ} \mathrm{C}\right)\end{array}$} & \multirow{2}{*}{$\begin{array}{c}\text { Ratio }_{\text {feed, }} \\
\text { mol\% } \\
(\text { GalX/ } \\
\text { diacid/ } \\
\text { diamine })\end{array}$} & \multicolumn{3}{|c|}{ GalXH } & \multicolumn{3}{|c|}{ GalXMe } \\
\hline $\begin{array}{c}\text { GalX- } \\
\text { salt }\end{array}$ & $\begin{array}{c}\text { Non- } \\
\text { GalX } \\
\text { salt }\end{array}$ & & & $\begin{array}{c}\text { Ratio, } \\
\text { mol\% } \\
\text { (GalX/ } \\
\text { diacid/ }^{\text {diamine) }}\end{array}$ & $\begin{array}{c}M_{n}{ }^{b} \\
\left(\mathrm{~kg}^{2} \cdot \mathrm{mol}^{-1}\right)\end{array}$ & $\boldsymbol{\boxplus}^{b}$ & $\begin{array}{c}\text { Ratio, } \\
\text { mol\% } \\
(\text { GalX/ } \\
\left.\text { diacid/ }^{\text {diamine }}\right)^{a}\end{array}$ & $\begin{array}{c}M_{n}^{b} \\
\left(\boldsymbol{k g} \cdot \mathrm{mol}^{-1}\right)\end{array}$ & $\boldsymbol{\Xi}^{b}$ \\
\hline \multirow{4}{*}{ 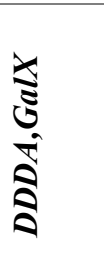 } & \multirow{4}{*}{$\frac{\pi}{2}$} & \multirow{4}{*}{$\begin{array}{l}220- \\
230\end{array}$} & $0 / 100 / 100$ & $0 / 100 / 93$ & 11.0 & 2.7 & $0 / 100 / 93$ & 11.0 & 2.7 \\
\hline & & & $10 / 90 / 100$ & 9/91/101 & 9.0 & 2.4 & 8/92/103 & 14.0 & 4.0 \\
\hline & & & $30 / 70 / 100$ & $27 / 73 / 105$ & 12.0 & 2.6 & $24 / 76 / 105$ & 21.0 & 6.0 \\
\hline & & & $50 / 50 / 100$ & $49 / 51 / 105$ & 16.0 & 2.7 & $44 / 56 / 105$ & 31.0 & 5.9 \\
\hline \multirow{4}{*}{ है } & \multirow{4}{*}{ 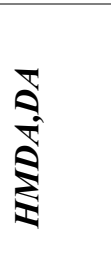 } & 230 & $0 / 100 / 100$ & $0 / 100 / 100$ & 6.6 & 2.2 & $0 / 100 / 100$ & 6.6 & 2.2 \\
\hline & & & $10 / 90 / 100$ & 8/92/103 & 6.2 & 2.7 & $3 / 97 / 105$ & 9.3 & 3.8 \\
\hline & & & $30 / 70 / 100$ & $24 / 76 / 105$ & 8.5 & 3.5 & $6 / 94 / 104$ & 9.9 & 6.5 \\
\hline & & & $50 / 50 / 100$ & $44 / 56 / 105$ & 7.0 & 3.8 & ND & 8.0 & 10.0 \\
\hline \multirow{4}{*}{ 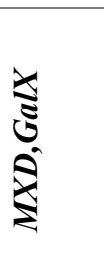 } & \multirow{4}{*}{$\frac{7}{8}$} & $220-$ & $0 / 100 / 100$ & $0 / 100 / 100$ & 8.8 & 2.2 & \multicolumn{3}{|c|}{ Degradation } \\
\hline & & 23 & $10 / 90 / 100$ & $8 / 92 / 97$ & 9.2 & 2.3 & \multicolumn{3}{|c|}{ Degradation } \\
\hline & & & $30 / 70 / 100$ & $26 / 74 / 101$ & 12.4 & 2.1 & \multicolumn{3}{|c|}{ Degradation } \\
\hline & & & $50 / 50 / 100$ & $50 / 50 / 88$ & 9.9 & 3.3 & \multicolumn{3}{|c|}{ Degradation } \\
\hline
\end{tabular}

ND: could not be determined. ${ }^{a}$ Determined by NMR ${ }^{b}$ Determined using GPC method with PMMA standards in HFIP

The above explanations, however, do not yet explain why polymerization resulted in fully degraded products with GalXMe salts in combination with MXD,DA salt, which is a low melting salt and should in principle give positive outcome of the polymerization. In order to better understand the polymerization behavior of the different systems, a closer look at the 
different salts is required. Therefore, TGA studies and optical microscopy studies were performed.

\subsection{Thermal stability of salts}

All GalX salts are high melting compounds with melting points above $220^{\circ} \mathrm{C}$ (see Figure 44) and tend to degrade during polymerization. In order to successfully polymerize GalX salts it is necessary to choose an appropriate non-GalX salt as a comonomer. It is hypothesized that the non-GalX salt acts as a solvent for the GalX salt, provoking polymerization in the molten medium below the melting point (which is also the start of degradation) of the GalX salt. In order to enable easy dissolution of the GalX salt and avoid thermal stresses on the GalX salt, low melting points of the non-GalX salts and big differences in melting points between the non-GalX and GalX salts are expected to be favorable. TGA results in Figure 44 systematically show that the GalXH salts are more thermally stable than the GalXMe salts, visible from the higher degradation temperatures of the GalXH salts.
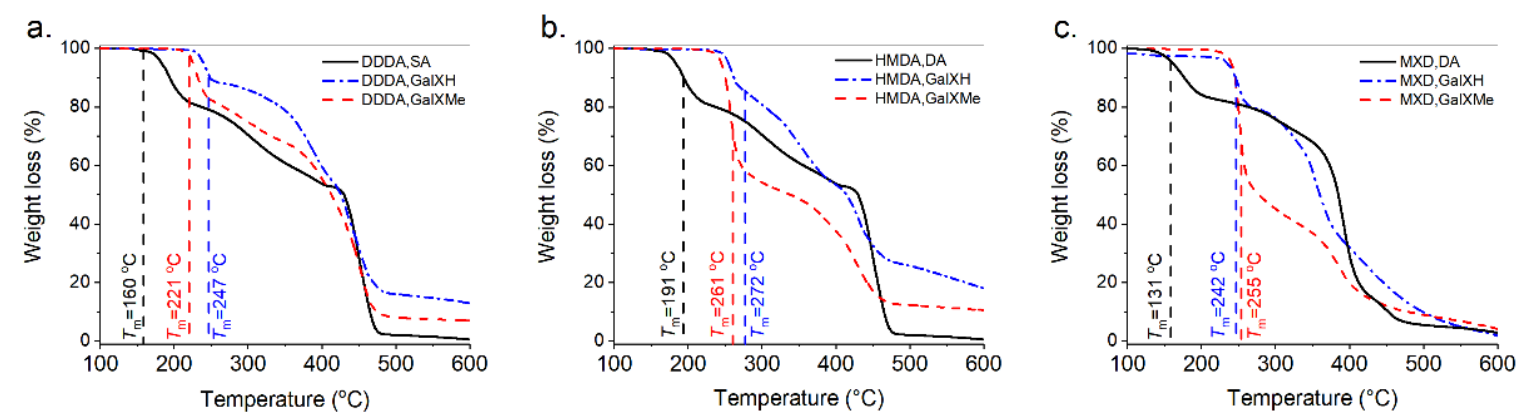

Figure 4-4 TGA profiles of GalX and non-GalX salts correlated with the $T_{m}$ of each salt (vertical values) for series (a) PA(DDDA,GalXR)-co-PA(DDDA,SA), (b) PA(HMDA,GalXR)-co-PA(HMDA,SA), (c) $P A(M X D, G a l X R)-c o-P A(M X D, D A), R=H$ for GalXH, $R=-C_{3}$ for GalXMe.

According to the above reasoning that the key to success is a big difference in melting points between the GalX and non-GalX salt, copolymerization of MXD,GalX salts with MXD,DA salts should work smoothly. However, the results in Table 4-1 reveal that only in case of GalXH salts copolymerization was successful, while with GalXMe salts degradation was observed. TGA analysis of the salts still does not explain why copolymerization of MXD,GalX salts with MXD,DA salts was not successful. Therefore, complementary microscopy studies were performed.

\subsection{Optical microscopy}

The system based on the DDDA,SA salt as non-GalX salt that gave successful polymerization (Figure 4-5 top) is compared with the system using MXD,DA salts (Figure 4- 


\section{Chapter 4}

5 bottom) for which polymerization only worked partially (only in combination with GalXH salts, not in combination with GalXMe salts). The different reaction possibilities as well as the melting points of the salts are summarized in Figure 4-5.

$$
\left.\prod_{\mathrm{O}}^{-}\right|_{8} ^{-} \prod_{\mathrm{O}}^{\mathrm{O}} \mathrm{H}_{3} \mathrm{~N}^{+}+\mathrm{N}_{12}^{+} \mathrm{NH}_{3}
$$$$
T_{\mathrm{m}}=160^{\circ} \mathrm{C}
$$<smiles>[NH3+]Cc1cccc(C[NH3+])c1</smiles><smiles>[R]C1([R])O[C@H](C(=O)[O-])[C@@H](C(=O)[O-])O[C@H]1C(=O)[O-]</smiles>

$\mathrm{R}=\mathrm{H}$ for DDDA, GalXH salt with $T_{\mathrm{m}}=247^{\circ} \mathrm{C}$ $\mathrm{R}=\mathrm{CH}_{3}$ for DDDA, GalXMe salt with $T_{\mathrm{m}}=221^{\circ} \mathrm{C}$<smiles>[R]C1([R])O[C@H](C(=O)[O-])[C@@H](C(=O)[O-])O[C@H]1C(=O)O</smiles>

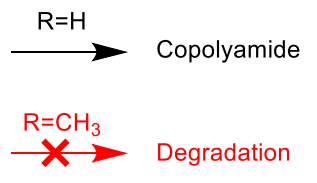

$\mathrm{R}=\mathrm{H}$ for MXD, GalXH salt with $T_{\mathrm{m}}=242^{\circ} \mathrm{C}$ $\mathrm{R}=\mathrm{CH}_{3}$ for MXD, GalXMe salt with $T_{\mathrm{m}}=255^{\circ} \mathrm{C}$

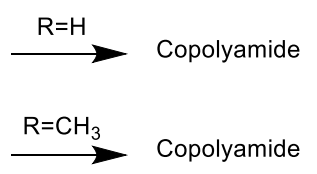

$\longrightarrow$ Degradation

Figure 4-5 The structure and melting points of salts used in the copolymerization reaction followed under optical microsope.

The microscopic observation of the PA(DDDA,SA) series (Figure 4-6) revealed that the GalX salt upon heating slowly dissolves in the non-GalX salt. For both GalXH and GalXMe, the crystals of their salts are vanishing already at a temperature below the melting point of those salts.
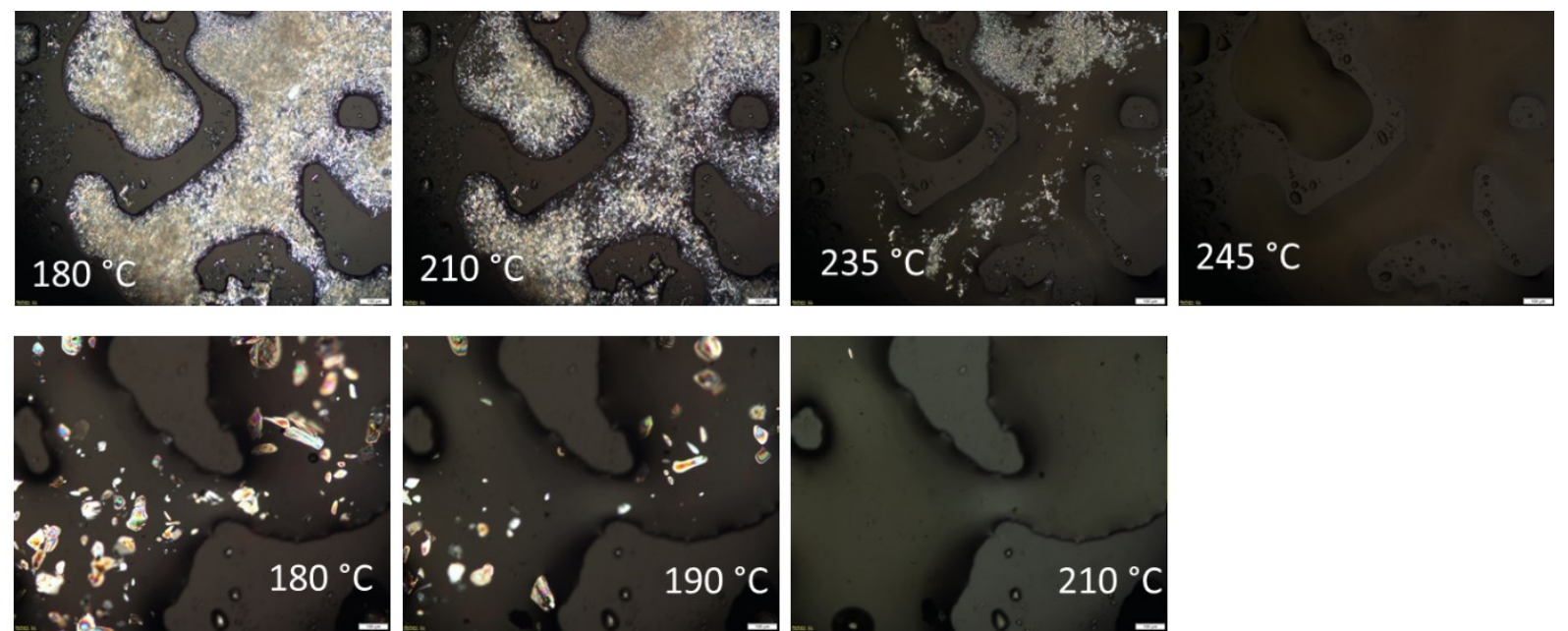

Figure 4-6 Optical microscopy images for heating a salt mixture containing equimolar amount of PA(12,10) and PA(12,GalX) salts. Top: GalXH, bottom: GalXMe (scale bar at right bottom represents $100 \mu \mathrm{m}$ ).

The crystals of the DDDA,GalXMe salt are fully molten/dissolved at $210{ }^{\circ} \mathrm{C}$ (Figure 4-6, bottom) which is $10^{\circ} \mathrm{C}$ below their melting point. The crystals of the DDDA,GalXH salt melt 
at higher temperatures so they are still visible at $235^{\circ} \mathrm{C}$, i.e. $20^{\circ} \mathrm{C}$ below their melting point (Figure 4-6, top), but they are quickly dissolving above that temperature. It seems that both salts are well soluble in and miscible with the molten medium of the non-GalX salt.

For the PA(MXD,DA) series only in case of GalXH the synthesis proved to have a positive outcome and with the GalXMe salt degraded product was obtained. Microscopy reveals different phenomena occurring during the melting process of the two GalX species.
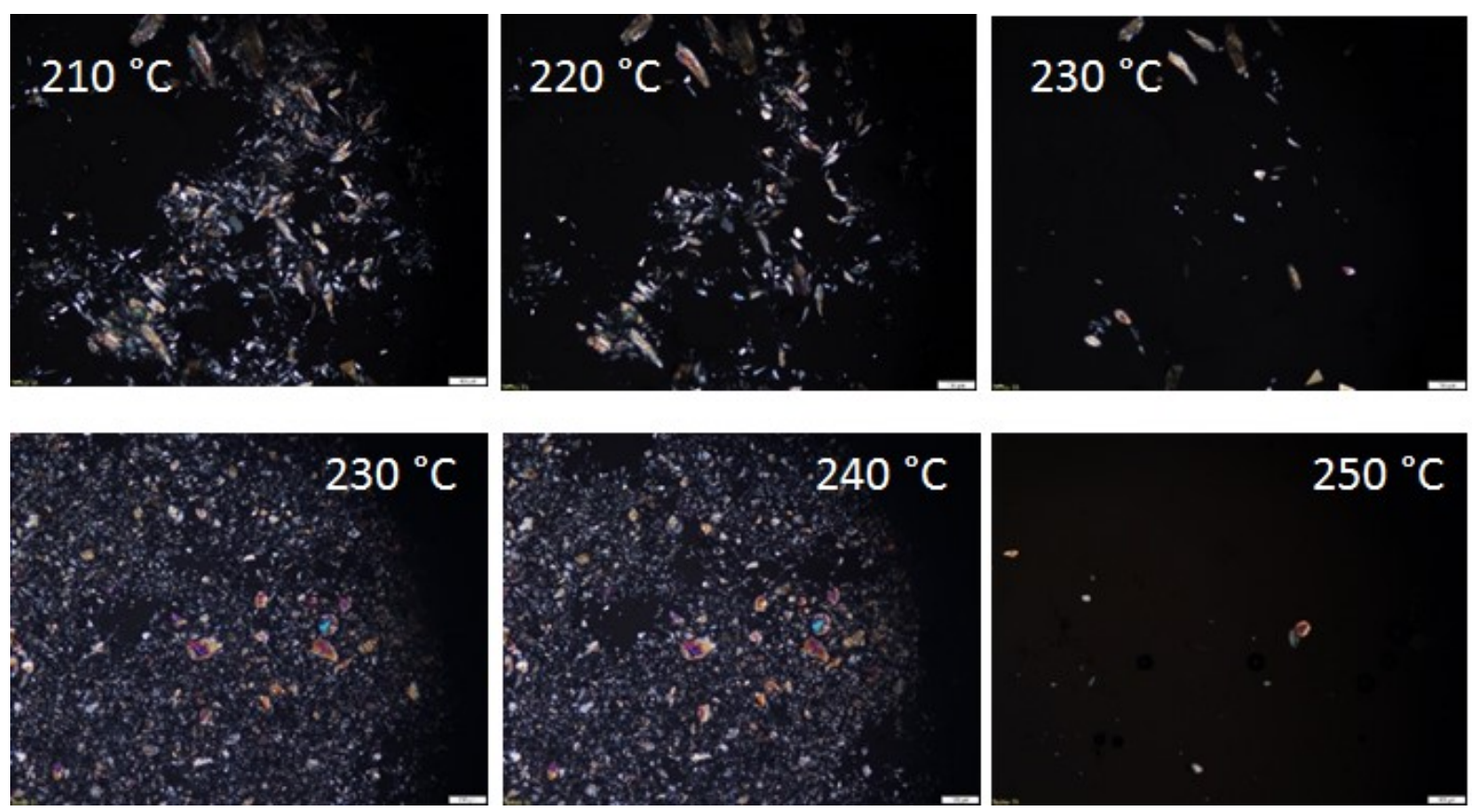

Figure 4-7 Optical microscopy images for heating a salt mixture containing equimolar amount of PA(MXD,12) and PA(MXD, GalX) salts. Top: GalXH, bottom: GalXMe (scale bar at right bottom represents $100 \mu \mathrm{m})$.

The microscopy images in Figure 4-7 (top) show that the MXD-GalXH crystals are dissolving slowly in the molten medium of the MXD-DA salt upon increasing temperatures. At $230{ }^{\circ} \mathrm{C}$ $\left(12{ }^{\circ} \mathrm{C}\right.$ below the melting point of the MXD-GalXH salt), the crystals are almost fully dissolved. Similar slow melting/dissolution is however not observed for the MXD-GalXMe salt crystals in the MXD,DA medium (Figure 4-7, bottom). Instead the MXD-GalXMe crystals disappear abruptly and almost completely at $250{ }^{\circ} \mathrm{C}$ which is $5{ }^{\circ} \mathrm{C}$ below the melting onset but already above the onset of the degradation presented in Figure 4-4(c). This indicates that the MXD-GalXMe salt is not soluble in the non-GalX salt MXD,DA, what explains why the copolymerization of those salts was not successful.

From the TGA and optical microscopy experiments some criteria for both salts can be deduced in order to give positive outcome of the polymer synthesis. The non-GalX salt needs to have a melting point which is preferably more than $30-50{ }^{\circ} \mathrm{C}$ lower that the GalX 


\section{Chapter 4}

containing salt and the molten medium of the non-GalX salt has to be a good solvent for the GalX salt. As such the GalX salt can dissolve into the non-GalX salt giving a homogeneous reaction mixture for polymerization without the need to heat the mixture up to the melting point of the GalX salt which is also its degradation temperature.

\subsection{Crystallinity}

In Figure 4-8 the photographs of PA(DDDA,SA) $100-\mathrm{x}-\mathrm{co}-(\mathrm{DDDA}, \mathrm{GalX})_{\mathrm{x}}$ polymer films casted from HFIP are presented. An increased amount of GalX moieties present in the polymer suppresses crystallinity of the material. The polymeric film containing $50 \%$ of GalXMe salt possesses characteristics of an amorphous material e.g. transparency and very weak or no melting point in the DSC. The corresponding polymer with $50 \%$ GalXH salt is partially crystalline, which indicates that GalXMe is suppressing crystallinity much more effectively than GalXH.
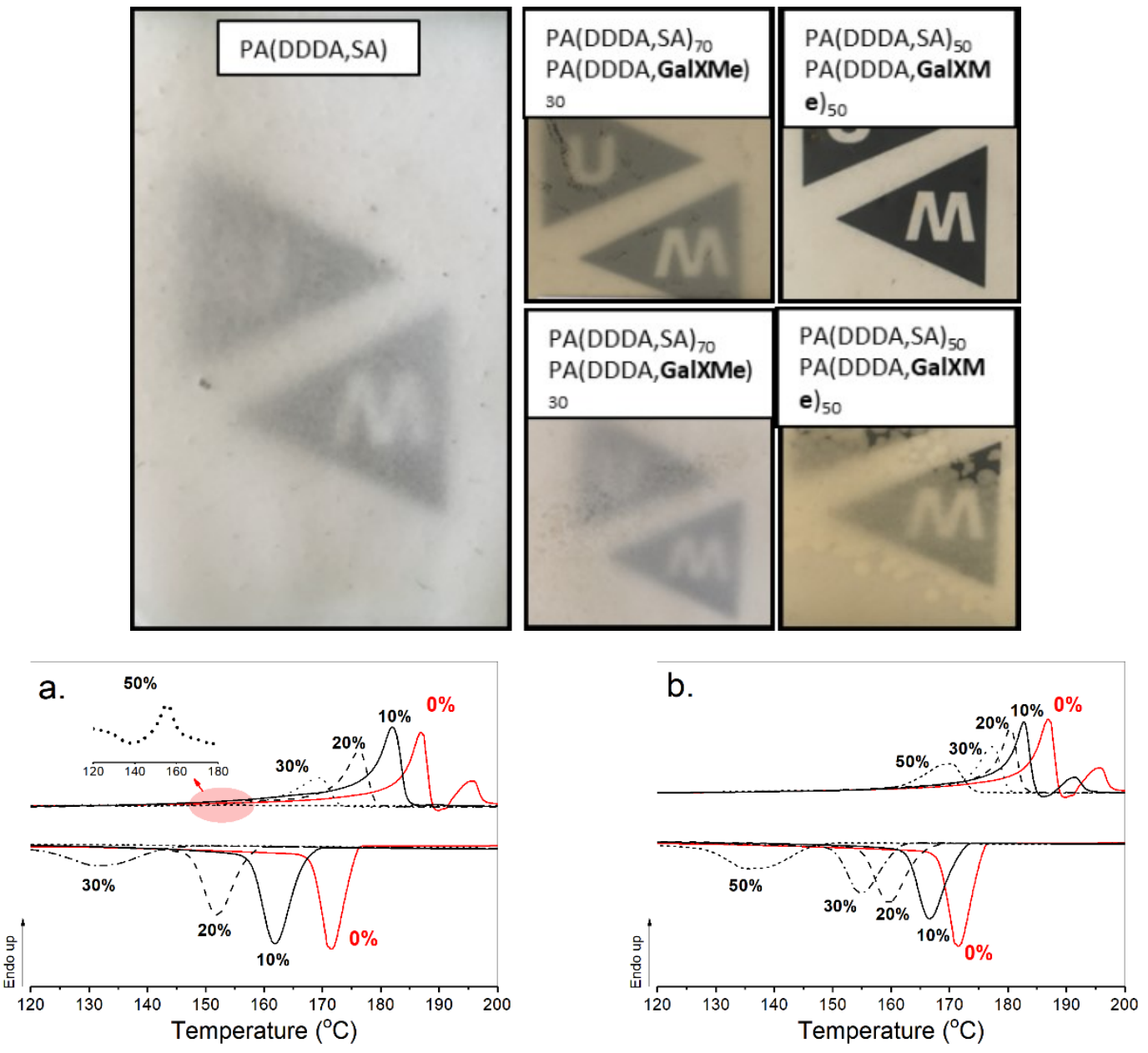

Figure 4-8 Photographs of PA(12,10) copolymer films casted from HFIP solution (top) and DSC traces of a. $P A(D D D A, S A)_{100-x}-c o-P A(D D D A, G a l X M e)_{x}$ and b. PA(DDDA, SA) $100-x-c o-P A(D D D A, G a l X H)_{x}$ recorded for different molar ratios of GalX and non-GalX component. 
The DSC results (Table 4-2) show a similar trend in crystallinity as was observed by macroscopic evaluation of the prepared films. The incorporation of GalX into the polymeric chain lowers the melting temperature and the crystallinity of the material and at the same time it has the exact opposite effect on the $T_{\mathrm{g}}$. The GalXMe is clearly a better crystallinity suppressor than GalXH and leads to almost amorphous transparent materials upon incorporation of $50 \%$ GalXMe into the PA(DDDA,SA) polyamide, which is also reflected by the very low melting enthalpy value. In the majority of the aliphatic polyamide series PA(DDDA,SA) and PA(HMDA,DA), the glass transition region was very poorly detectable by means of DSC, nonetheless, the collected data revealed that the glassy-rubbery transition stays below $55{ }^{\circ} \mathrm{C}$. When GalX salts were combined with MXD,DA salts, polymers with elevated $T_{\mathrm{g}}$ could be obtained. The incorporation of $50 \%$ of GalXH into the polymeric structure resulted in an increase of the $T_{\mathrm{g}}$ by $24{ }^{\circ} \mathrm{C}$ and effective suppression of crystallization.

Table 4-2 Thermal characteristics of the prepared copolyamides as determined by DSC (heating and cooling rate of $10^{\circ} \mathrm{C} / \mathrm{min}$ )

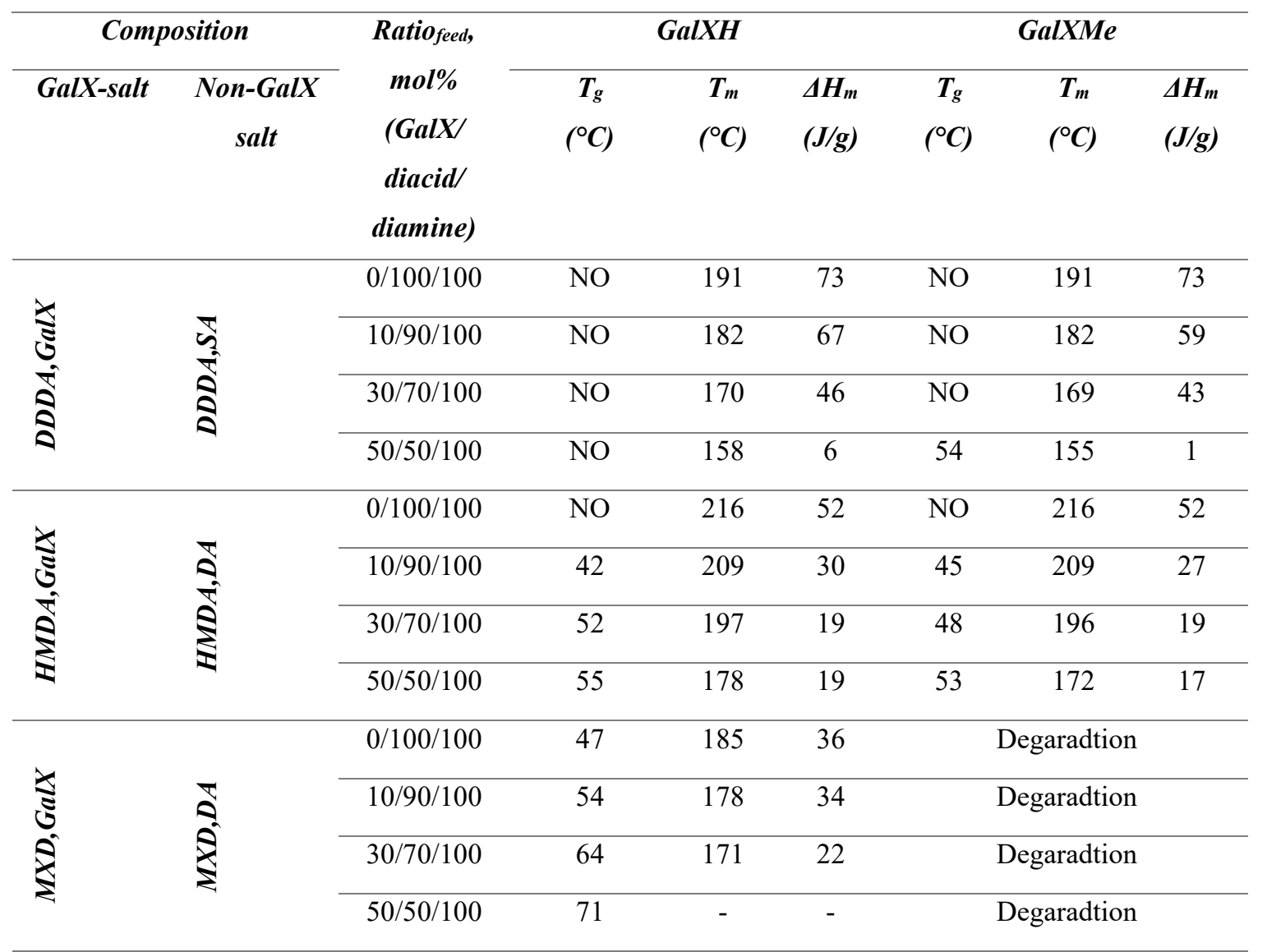

NO: not observed 


\section{Chapter 4}

\subsection{Maldi-ToF of low molecular weight copolymers}

Maldi-ToF was used to confirm phenomena described earlier (GalX incorporation or degradation) and to prove that the polymerization resulted in the formation of copolymeric chains and not in two homopolymers. The polymers for Maldi-ToF analysis were prepared intentionally with an excess of aliphatic diacid in order to keep the molecular weight low and have good resolution of the mass spectra.

The data are presented in the form of contour plots, ${ }^{46,47}$ which allow for quick and straightforward identification of homopolymeric and copolymeric chains in the sample. The contour plots have on the $\mathrm{X}$ axis the amount of repeating units of one monomer (in this case non-GalX repeating unit $\mathrm{x}$ ) and on the $\mathrm{Y}$ axis the amount of the other (in this case GalX repeating units y) incorporated into the polymer. Essentially, the Maldi-ToF spectrum of homopolymers is located on the $\mathrm{X}$ or $\mathrm{Y}$ axis $(\mathrm{Y}=0$ or $\mathrm{X}=0)$ of the two dimensional contour plot and copolymers are in the area between the axis $(X>0$ and $Y>0)$. The shape and the size of the graph delivers information about the ratio of the monomers, the reactivity ratio of monomers and the microstructure (the presence of homopolymeric moieties, the presence of block copolymers etc.). In principle, a contour plot of alternating copolymer is symmetrical to the diagonal. When the copolymer has one block of different length or one monomer is in excess the graph is no longer symmetrical and the plot shifts towards the axis which corresponds to the repeating unit which occurs in excess.

Contour plots were prepared for each identified distribution of the copolymers: linear diacid terminated distribution $\alpha-\mathrm{HO}-\mathrm{x} R U_{x}-\mathrm{y} R U_{y}-\mathrm{CO}-\mathrm{C} 8-\mathrm{COOH}$, cyclic distribution $\beta-\mathrm{x} R U_{x^{-}}$ $\mathrm{y} R U_{y^{-}}$and linear acid / amine terminated distribution $\gamma$ - HO-x $R U_{x}-\mathrm{y} R U_{y^{-}} \mathrm{H}$ (see Figure 4-9). 


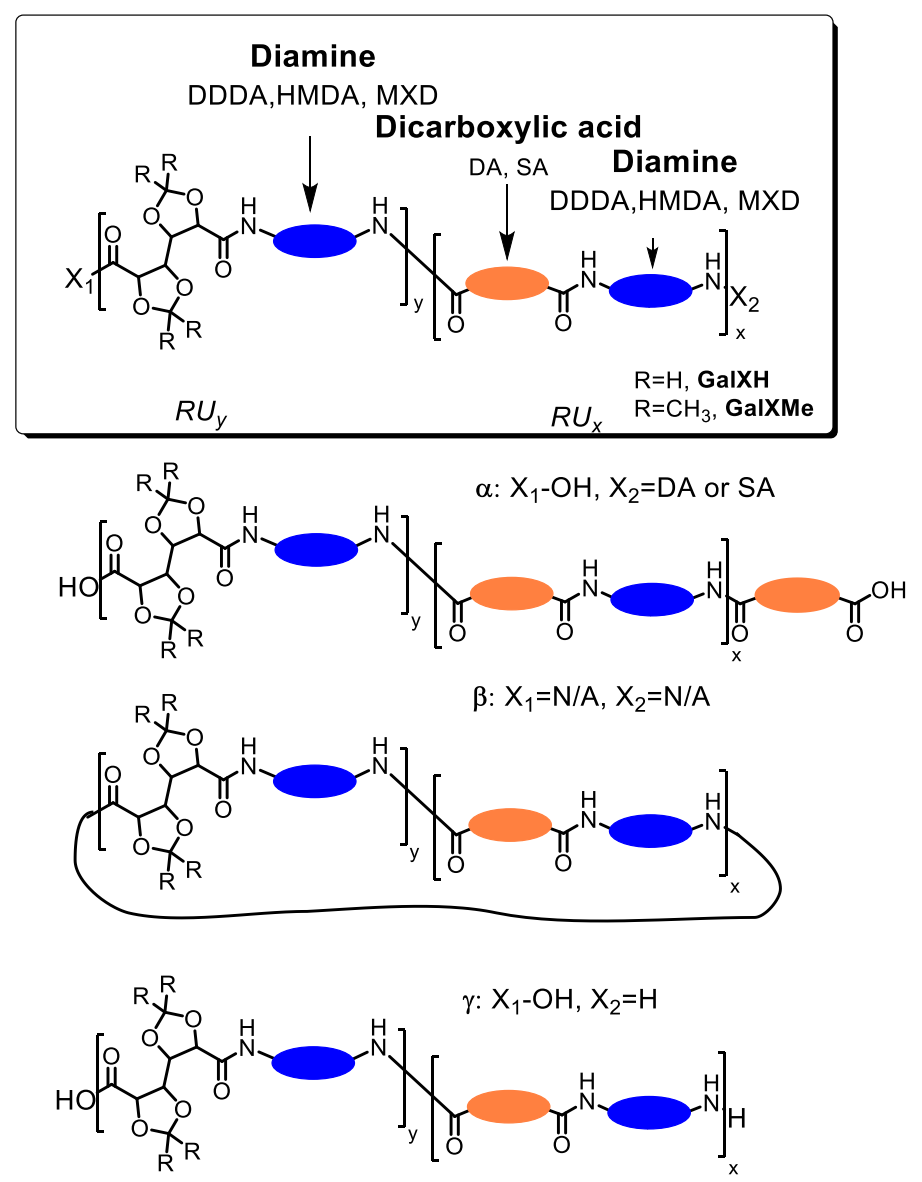

Figure 4-9 General structures of the three main distributions identified in the samples of low molecular weight copolyamides.

The analysis of the Maldi-ToF spectrum of the DDDA,SA salt copolymerized with the DDDA,GalXH salt revealed the presence of two major distributions $\alpha$ and $\beta$, corresponding to acid terminated (distribution $\alpha$ Figure 4-10 (a)) and cyclic (distribution $\beta$ Figure 4-10(b)) chains. Additionally, several small distributions are present in the spectrum among which amine and acid terminated linear chains, distribution $\gamma($ Figure 4-10(c)). For the distributions $\alpha, \beta$ and $\gamma$ (Figure 4-10(a), (b) and (c)) the formation of significant amounts of both homopolymers, PA(DDDA,GalX) $(\mathrm{X}=0)$ and PA(DDDA,GalXH) $(\mathrm{Y}=0)$, are revealed next to copolymers. 


\section{Chapter 4}
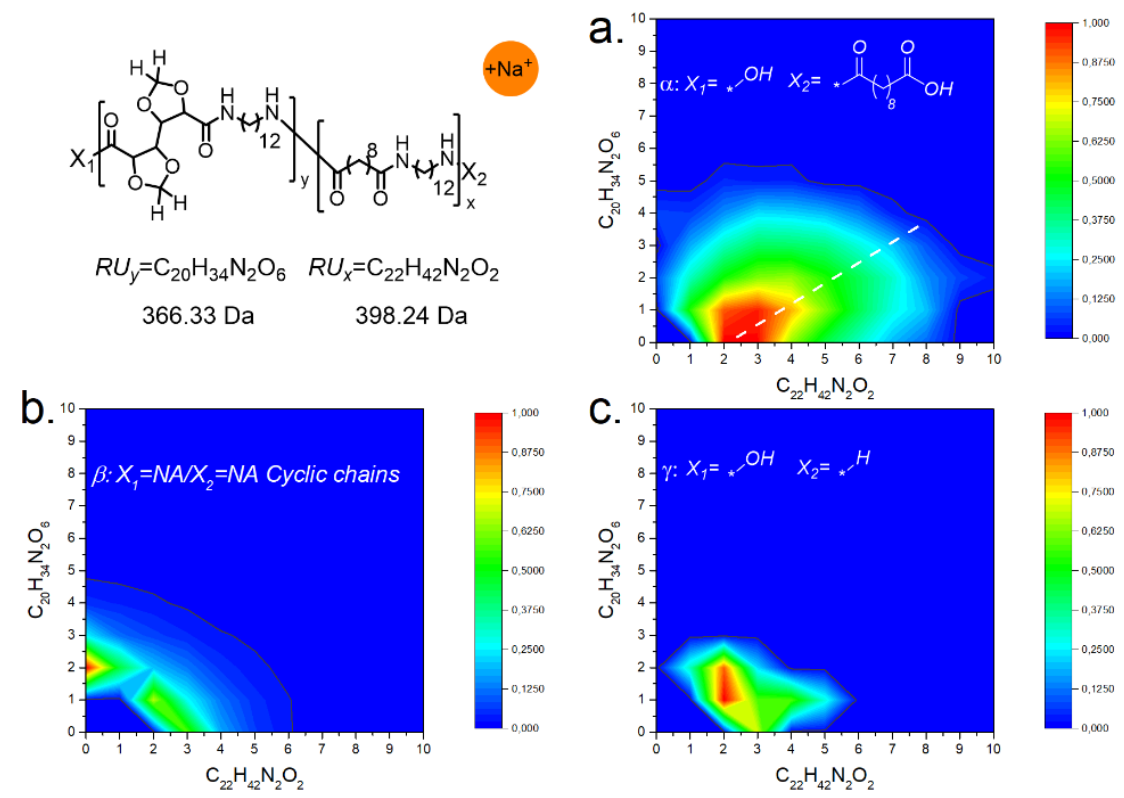

Figure 4-10 Contour plot Maldi-ToF spectrum of low molecular weight PA(DDDA,GalXH) $)_{30}$ co$P A(D D D A, S A)_{70}\left(M_{n, G P C}=8.1 \mathrm{~kg} / \mathrm{mol}, \emptyset=2.6\right)$. (a) acid end groups, (b) cyclic chains, (c) amine and acid end groups

The predominant presence of the distribution with end groups corresponding to acid terminated polymeric chains $\alpha$ Figure 4-10(a) can be explained by the addition of an excess of SA diacid during the preparation of the polymers in order to keep the molecular weight low for Maldi-ToF analysis. The Maldi-ToF contour plot is very asymmetric with respect to the diagonal and indicates an excess of non-GalX repeating units DDDA-SA in the copolymer which originates from the molar ratio of the salts 30 eq. GalX salt : 70 eq. non-GalX salt. The position of the contour plot with respect to the diagonal for the same polymer with a molar ratio 50 eq. GalX salt : 50 eq. non-GalX salt in the feed is more symmetric (Figure 4-11). The analysis further confirmed that also in this case both homopolymers, PA(DDDA-GalX) $(\mathrm{X}=0)$ and $\mathrm{PA}(\mathrm{DDDA}-\mathrm{GalXH})(\mathrm{Y}=0)$, are present.
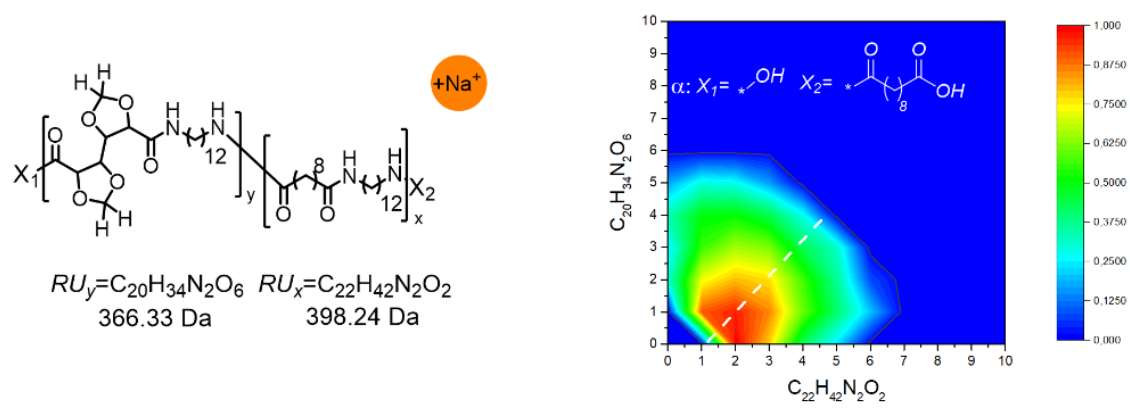

Figure 4-11 Contour plot Maldi-ToF spectrum for distribution $\alpha$ (with acid end groups) of low molecular weight PA(DDDA, GalXH) $)_{50}$-co-PA(DDDA,SA $)_{50}\left(M_{n, G P C}=7.4 \mathrm{~kg} / \mathrm{mol}, \emptyset=2.7\right)$. 
The contour plot of PA(DDDA,GalXMe) ${ }_{30}-c o-P A(D D D A, S A) 70$ Figure 4-11 shows a similar trend as the previously discussed spectrum (Figure 4-11) for the corresponding GalXH polymer with this difference that almost no homopolymeric chains of PA(DDDA,GalXMe) are present in the linear distribution $\alpha$ or cyclic distribution $\beta(Y>0)$ (Figure 4-12 (a) and (b)). The spectrum is asymmetric towards a higher content of aliphatic moieties, almost parallel to the $\mathrm{X}$ axis, which relates to the formation of block copolymeric structures, rich in aliphatic

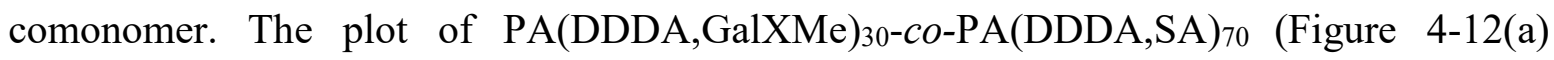
distribution $\alpha$ ) is more shifted towards the $\mathrm{X}$ axis than the earlier presented corresponding plot for GalXH (PA(DDDA,GalXH) $30-c o-P A(D D D A, S A)_{70}$ in Figure 4-10 (a), distribution $\alpha$ ), which presumably is a result of the inferior incorporation of GalXMe into copolymeric chains in comparison to GalXH monomer.

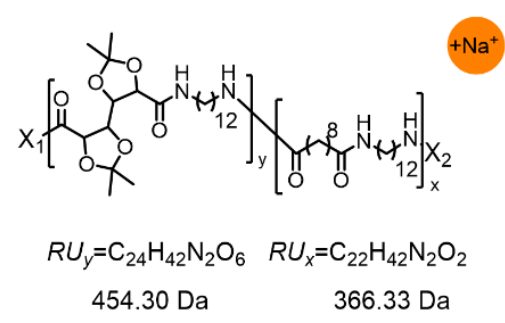

a.

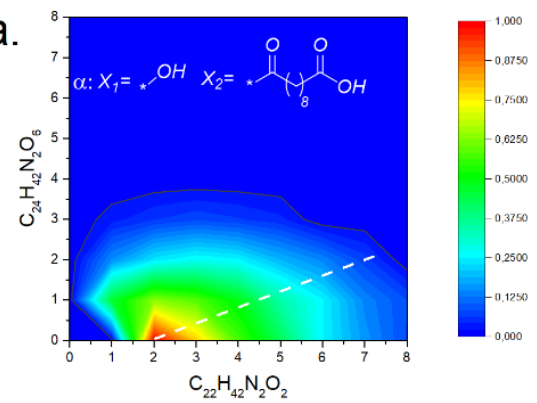

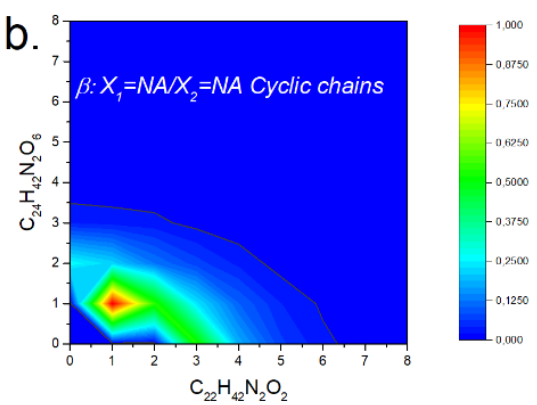

Figure 4-12 Contour plot Maldi-ToF spectrum of low molecular weight PA(DDDA, GalXMe) $)_{30-c o-}$ $P A(D D D A, S A)_{70}\left(M_{n, G P C}=11.6 \mathrm{~kg} / \mathrm{mol}, \oplus=8.7\right)$. (a) acid end groups, (b) cyclic chains.

The Maldi-ToF contour plot in Figure 4-13 confirms the incorporation of the HMDAGalXMe salt in the copolyamide, however to a very limited extent. Chains rich in aliphatic monomers under the form of homopolymers and copolymers are dominating Figure 4-13 (a and b). As mentioned earlier, GalXMe is susceptible to degradation induced by high temperature and acidic groups. This phenomenon is more severe in less hydrophobic reaction media, as confirmed by the fact that the cloud of PA(HMDA,GalXMe) $30-c o-$ PA(HMDA,DA) 70 (Figure 4-13(a)) is smaller than the one of PA(DDDA,GalXMe) $30-c o-$ PA(DDDA,SA)70 Figure 4-12(a)). All those aspects lead to the conclusion that 


\section{Chapter 4}

copolymerization of HMDA-GalXMe salt and HMDA-DA salt is possible but the incorporation of GalXMe is limited due to presumably GalX degradation processes which can be dominating over proper polycondensation.

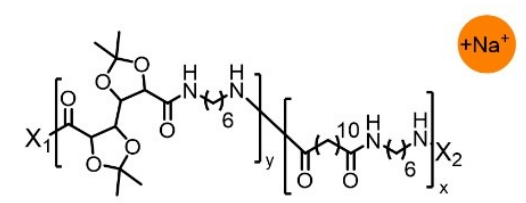

$$
\begin{array}{cc}
R U_{y}=\mathrm{C}_{24} \mathrm{H}_{30} \mathrm{~N}_{2} \mathrm{O}_{6} & R U_{x}=\mathrm{C}_{18} \mathrm{H}_{34} \mathrm{~N}_{2} \mathrm{O}_{2} \\
370.21 \mathrm{Da} & 310.26 \mathrm{Da}
\end{array}
$$
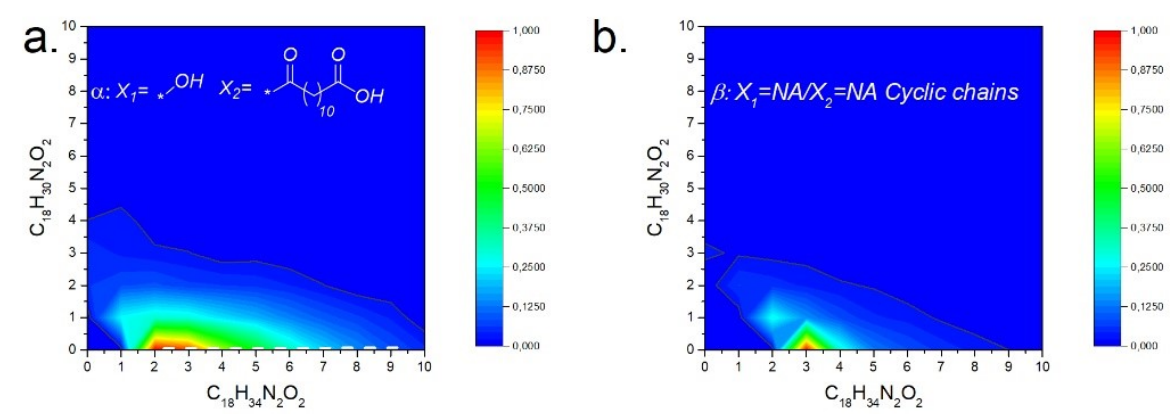

Figure 4-13 Contour plot Maldi-ToF spectrum of low molecular weight PA(HMDA,GalXMe) $30-$ co$P A(H M D A, D A 2)_{70}\left(M_{n, G P C}=5.5 \mathrm{~kg} / \mathrm{mol}, \emptyset=5.3\right)$.

\subsection{Temperature dependent FT-IR.}

To elucidate the processes occurring during polymerization FT-IR spectra were collected. A previously prepared equimolar mixture of two salts GalX salt and non-GalX salt were placed on the ATR crystal at $160{ }^{\circ} \mathrm{C}$, heated to $220^{\circ} \mathrm{C}$, followed by an isothermal program at $220^{\circ} \mathrm{C}$. The results are shown in Figure 4-14 and Figure 4-15 

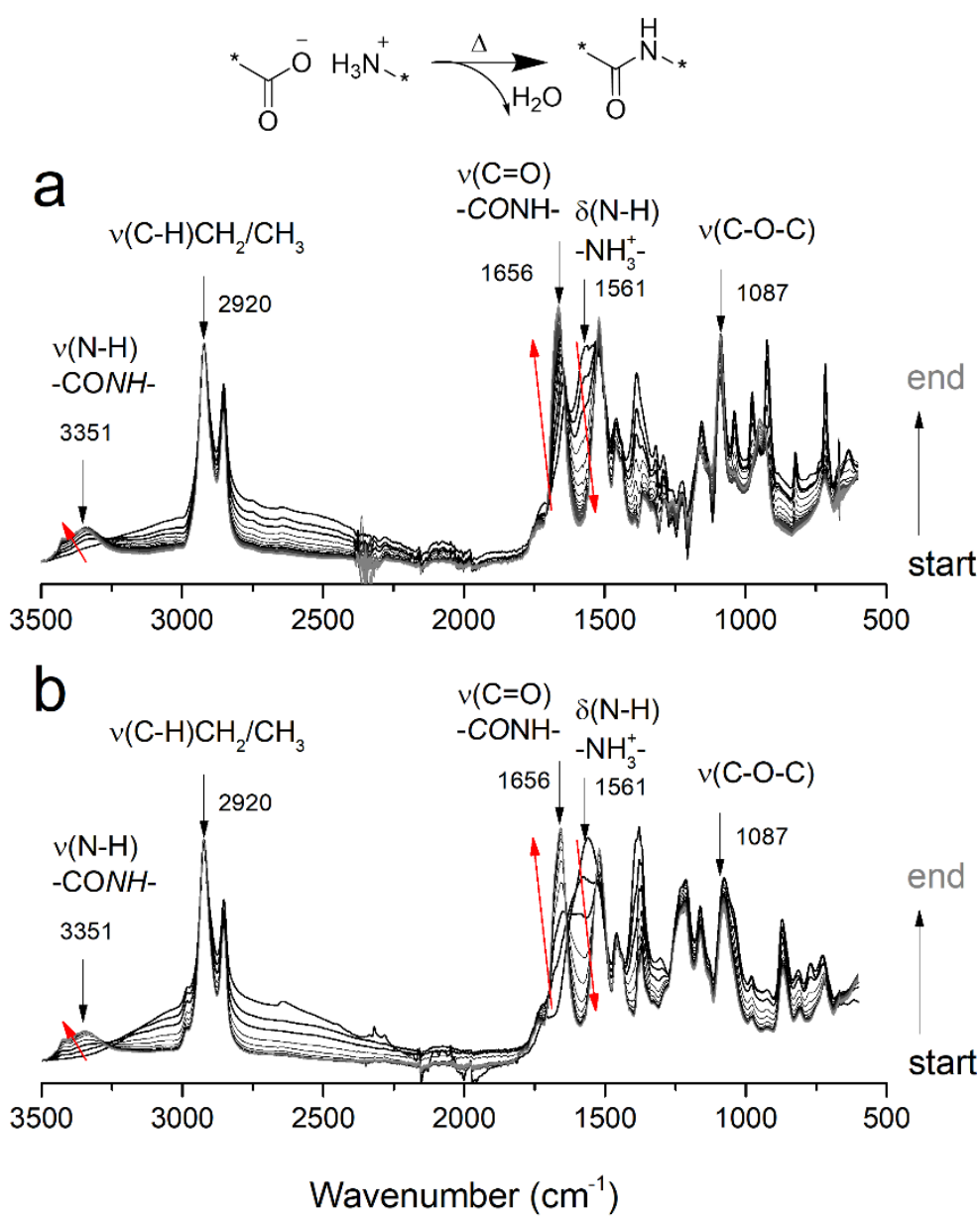

Figure 4-14 Time depended FT-IR spectrum recorded for reaction of (a) DDDA,SA salt with DDDA, GalXH salt (ratio 50:50) and (b) DDDA, SA salt with DDDA, GalXMe salt (ratio 50:50) and a general reaction scheme (top). The bands demonstrate the followed vibrations $\left(3351 \mathrm{~cm}^{-1}, 1656 \mathrm{~cm}^{-1}, 1561 \mathrm{~cm}^{-1}\right.$ and $\left.1087 \mathrm{~cm}^{-1}\right)$ and the normalization peak at $2920 \mathrm{~cm}^{-1}$. $\delta$-bending (scissoring), $v$-stretching vibration. Red arrows indicate the development of the signal along the reaction coordinates.

For both sets of data a kinetic analysis shows a typical reactivity for step-growth polymerization: the majority of the functional groups of the substrates $\left(\delta(\mathrm{N}-\mathrm{H}) \mathrm{NH}_{3}{ }^{+}\right)$have already reacted in an early stage of the experiment (Figure 4-14). Initially, a band corresponding to the bending vibration of $\mathrm{N}-\mathrm{H}$ in the salt is present at $1561 \mathrm{~cm}^{-1}$, but along the reaction progress this band rapidly disappears and stretching bands of N-H amide at 3351 $\mathrm{cm}^{-1}$ and of the carbonyl in the amide at $1656 \mathrm{~cm}^{-1}$ become predominant (see Figure 4-14). PA(HMDA,DA) $50-c o-P A(H M D A, G a l X H)_{50}$ in Figure 4-14(a) reaches a plateau later than the other two reactions due to the higher melting point of the used salts (see Figure 4-4).

The band corresponding to the stretching vibration of the C-O-C fragment of the acetal moieties in GalX around $1087 \mathrm{~cm}^{-1}$ is followed to investigate whether the GalX acetal undergoes any temperature induced transformations. The $v(\mathrm{C}-\mathrm{O}-\mathrm{C})$ region at $1087 \mathrm{~cm}^{-1}$ in Figure 4-15(c) and (d) shows a slight slope at the beginning of the experiment due to lowering 


\section{Chapter 4}

of the baseline originating from the melting processes of the salts. The FT-IR results in Figure 4-15(c) and (d) confirm the GalX stability results from Table 4-1 and Figure 4-3: thermally induced and acid catalyzed (due to the acid end groups of GalX) deprotection of acetals is much more pronounced for GalXMe than for GalXH. Figure 4-15(d) shows that for PA(MXD,DA)50-co-PA(MXD,GalXMe)50 immediate acetal degradation happens (conclusions from optical microscopy in Figure 4-7), for PA(HMDA,DA)50-coPA(HMDA, GalXMe)50 also pretty rapid acetal degradation can be observed and for the most hydrophobic PA(12,10)50-co-PA(12,GalXMe)50 the degradation of acetal has the slowest rate (berely visible). For PA(HMDA,DA)50-co-PA(HMDA,GalXH)50 the acetal signal is increasing after the aliphatic salt is dissolved (Figure 4-15 c), indicating that the GalXH salt is slowly dissolving in the aliphatic salt. For GalXH acetal we also observe a slight decrease of the intensity after the dissolution, which could be related to the degradation or just simple sublimation of the GalX acids during the experiment.
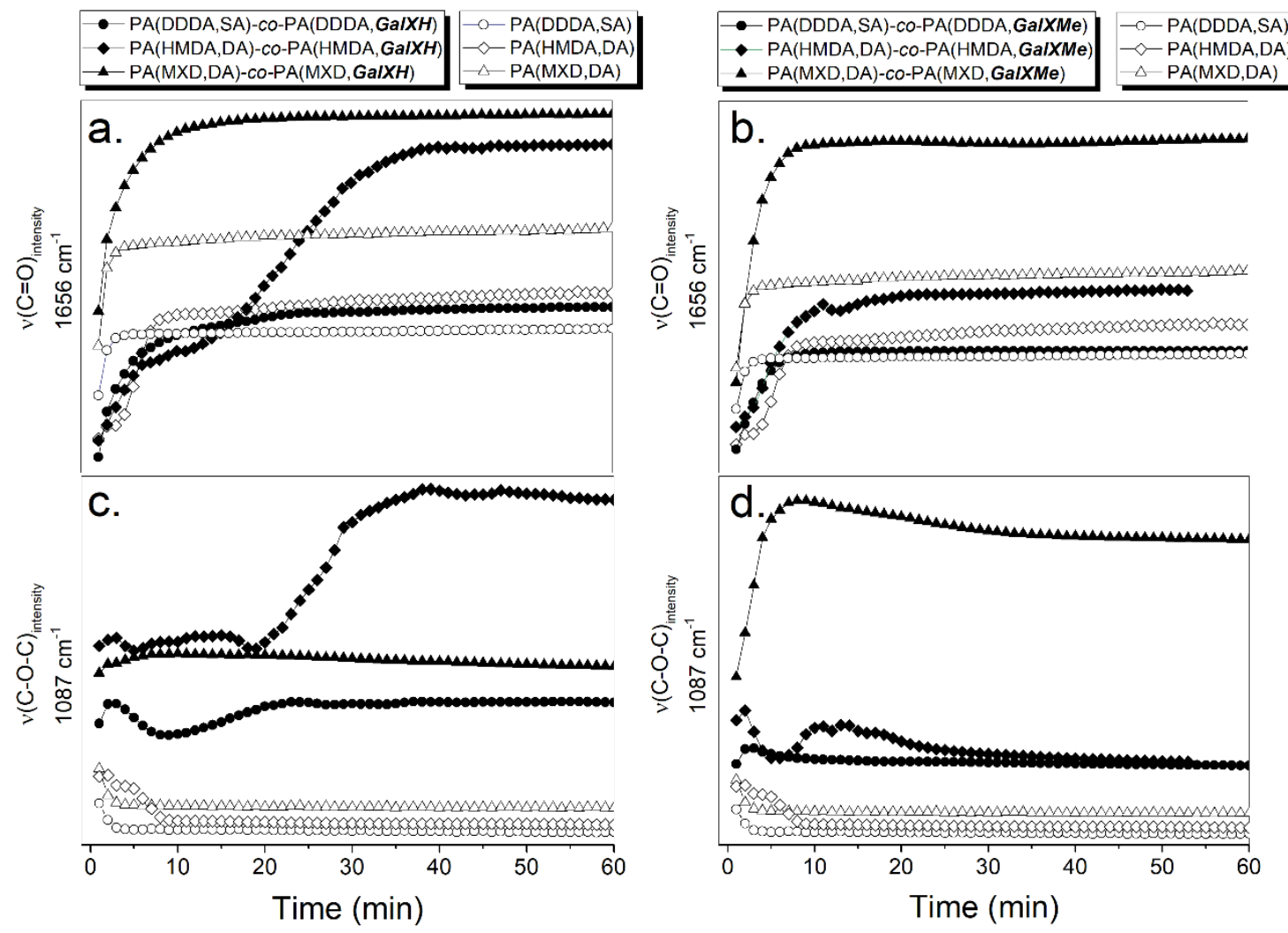

Figure 4-15 The intensity of (a) $v(C=O)$ band at $1656 \mathrm{~cm}^{-1}$ of GalXH copolymers, (b) $v(C=O)$ band at 1656 $\mathrm{cm}^{-1}$ of GalXMe copolymers, (c) v(C-O-C) band at $1087 \mathrm{~cm}^{-1}$ of GalXH copolymers and (d) v(C-O-C) band at $1087 \mathrm{~cm}^{-1}$ of GalXMe copolymers as a function of the reaction time for equimolar mixtures of salts. 


\subsection{Conclusions}

The modified solvent free melt polycondensation of polyamide salts investigated mechanistically in this article enabled for the first time the successful polymerization of sugar-derived GalX monomers resulting in fully or partially biobased polyamides. The positive outcome of this modified polycondensation method is dependent on the miscibility/solubility of salt monomers, the temperature difference between their melting points and the hydrophobicity of the reaction medium, however does not depend on the melting temperature of the used sugar-derived monomer itself. The incorporation of two biacetalized sugar-derived monomers GalXH and GalXMe into polyamides was compared and the crystallinity of the copolymers can be tuned via the choice of the GalX species and via the composition of the polyamide. GalXH and GalXMe have different sensitivity to thermal and acidic degradation of the cyclic acetal moieties. The GalXMe salt is more challenging to incorporate via melt polycondensation than the GalXH salt due to acid (coming from the acid end groups) induced deprotection of the acetal. However, in the presence of hydrophobic comonomers, successful incorporation of GalXMe in copolyamides was confirmed by Maldi-ToF and NMR. We expect this technique to be broadly applicable not only for GalX polymerization but also for other labile biobased monomers.

\subsection{Materials and methods}

\subsubsection{Materials}

Diethyl 2,3:4,5-di- $O$-isopropylidene-galactarate $>99 \%$, diethyl 2,3:4,5-di- $O$-methylenegalactarate $>99 \%, 2,3: 4,5$-di- $O$-isopropylidene-galactaric acid $>99 \%$ and 2,3:4,5-di-Omethylene-galactaric acid $>99 \%$ were supplied by Royal Cosun. 1,6-hexamethylenediamine (HMDA) 98\%, 1,12-dodecandiamine (DDDA) 98\%, dodecanedioic acid (DA) 98\%, decanedioic acid (C10 - SA) 98\%, 1,3-phenylenedimethanamine (MXD) 98\%, Irganox 1330, pentafluorophenol- $\mathrm{d}_{1}$ 98\% (PFP), phenol 99\%, dithranol, NaTFA were purchased from Sigma-Aldrich and used as supplied. 1,1,1,3,3,3-hexafluoro-2-propanol (HFIP), 99.7\% DMSO- $\mathrm{d}_{6}, \mathrm{CDCl}_{3}, \mathrm{D}_{2} \mathrm{O}$ were purchased from Acros Organics. Acetone was purchased from Biosolve.

\subsubsection{Methods}

1,6-hexamethylenediamine-2,3:4,5-O-isopropylidene-galactaric acid salt. To a solution of 2,3:4,5-O-isopropylidene-galactararic acid (4.35 g, $15 \mathrm{mmol})$ in ethanol $(10 \mathrm{~mL})$ at $50{ }^{\circ} \mathrm{C}$, 
1,6-hexamethylenediamine $(1.74 \mathrm{~g}, 15 \mathrm{mmol})$ in an ethanol $(6 \mathrm{~mL})$ was added dropwise. During the addition, a precipitate was formed. The mixture was stirred at $80{ }^{\circ} \mathrm{C}$ for $2 \mathrm{~h}$. The crude product was filtered and recrystallized from an ethanol/water mixture $(8 / 1, \mathrm{v} / \mathrm{v})$ to result in the salt as white crystals $\left(3.83 \mathrm{~g}, 86 \%\right.$ yield) with a melting point of $261{ }^{\circ} \mathrm{C}$.

${ }^{1} \mathrm{H} \mathrm{NMR}\left(300 \mathrm{MHz}, \mathrm{D}_{2} \mathrm{O}\right) \delta$ (ppm): 4.50 (2H, -CH-O-, d), 4.44 (2H, -CH-O-, d), 2.91 (4H, $\mathrm{CH}_{2}-\mathrm{NH}_{3}{ }^{+}$t). $1.58\left(4 \mathrm{H},-\mathrm{CH}_{2}-\mathrm{CH}_{2}-\mathrm{NH}_{3}{ }^{+}, \mathrm{HMDA}, \mathrm{m}\right), 1.42\left(6 \mathrm{H},-\mathrm{CH}_{3}, \mathrm{GalX}, \mathrm{s}\right), 1.34(6 \mathrm{H},-$ $\left.\mathrm{CH}_{3}, \mathrm{GalX}, \mathrm{s}\right), 1.26\left(4 \mathrm{H},-\mathrm{CH}_{2}-\mathrm{CH}_{2}-\mathrm{CH}_{2}-\mathrm{NH}_{3}{ }^{+}, \mathrm{HMDA}, \mathrm{m}\right)$

ATR-IR v $\left(\mathrm{cm}^{-1}\right): 3500-3000 v(\mathrm{~N}-\mathrm{H}) \mathrm{NH}_{3}{ }^{+}, 2986 v(\mathrm{C}-\mathrm{H}) \mathrm{CH}_{3}, 2933 v(\mathrm{C}-\mathrm{H}) \mathrm{CH}_{2}$ antisym., $2859 v(\mathrm{C}-\mathrm{H}) \mathrm{CH}_{2}$ sym., $1561 \delta(\mathrm{N}-\mathrm{H}) \mathrm{NH}_{3}{ }^{+}, 1455 \delta(\mathrm{C}-\mathrm{H}) \mathrm{CH}_{2}, 1161 / 1079 v(\mathrm{C}-\mathrm{O}-\mathrm{C})$.

The other salts were prepared in a similar manner.

Poly(1,6-hexamethylenediamine-1,12-dodecanedioate)-co-poly(1,6-hexamethylenediamine2,3:4,5-O-isopropylidenegalactarate). To a $100 \mathrm{~mL}$ three-necked round bottom flask (equipped with a vacuum-tight mechanical stirrer, a Vigreux column and a distillation condenser) were added 1,6-hexamethylenediamine-2,3:4,5-O-isopropylidene-galactaric acid salt (4.48 g, $15 \mathrm{mmol})$, 1,6-hexamethylenediamine-1,12-dodecandioic acid salt (5.20 g, 15 mmol) and antioxidant Irganox 1330 (0.01 g, $0.1 \mathrm{wt} \%)$. The reactants were slowly heated till $220-230{ }^{\circ} \mathrm{C}$ (above the melting point of the aliphatic salt). After all water had been fully distilled off, vacuum was applied for 1-3 h. The crude product was obtained as a yellowish material.

Other copolyamides were prepared in a similar manner, with specific reaction temperatures mentioned in Table 4-1.

Low molecular weight poly(1,6-hexamethylenediamine-1,12-dodecanedioate)-co-poly(1,6hexamethylenediamine-2,3:4,5-O-isopropylidenegalactarate). To a $5 \mathrm{~mL}$ crim-top vial were added 1,6-hexamethylenediamine-2,3:4,5-O-isopropylidene galactaric acid salt $(0.500 \mathrm{~g}$, $1.67 \mathrm{mmol})$, 1,6-hexamethylenediamine-1,12-dodecandioic acid salt (0.508 g, $1.67 \mathrm{mmol})$ with an excess of 1,12-dodecanedioic acid $(8.0 \mathrm{mg}, 0.03 \mathrm{mmol})$ to control the molecular weight. The vials were flushed with nitrogen for $2 \mathrm{~h}$ and then slowly heated till $220-230{ }^{\circ} \mathrm{C}$ (above the melting point of the aliphatic salt). The crude product was obtained as a yellowish material.

Thermal stability of monomers. Approximately $1 \mathrm{~g}$ of each monomer (2,3:4,5-Oisopropylidene-galactaric acid, 2,3:4,5-O-methylene-galactaric acid, diethyl 2,3:4,5-Oisopropylidene-galactarate and diethyl 2,3:4,5-O-methylene-galactarate) was placed in a 
crimp-top vial and flushed with nitrogen. The vials were quickly heated to $220{ }^{\circ} \mathrm{C}$ and kept at this temperature for 5 minutes.

\subsubsection{Characterization}

The thermal stability, melting temperatures, DSC thermograms, molecular weight of polyamides, liquid chromatography - mass spectrometry, matrix-assisted laser desorption/ionization time-of-flight (Maldi-ToF) were determined/recorded as described in the experimental section of Chapter 2.

Temperature - dependent infrared spectroscopy (Td-FTIR) was recorded on a PerkinElmer FTIR/NIR spectrometer Frontier with resolution $4 \mathrm{~cm}^{-1}$ and 8 accumulations per spectrum. Two salts were mixed together in acetone and the sample was left overnight in order to allow the solvent to evaporate. Around $5 \mathrm{mg}$ of the sample was heated from $160{ }^{\circ} \mathrm{C}$ to $220^{\circ} \mathrm{C}$ with the heating rate $10{ }^{\circ} \mathrm{C} / \mathrm{min}$ and spectra were recorded at time intervals of 1 minute over the course of 100 minutes. The background scan was recorded at $220{ }^{\circ} \mathrm{C}$ with 64 accumulations at a resolution $4 \mathrm{~cm}^{-1}$. The recorded spectra were normalized to the stretch vibration of the methylene group at $2920 \mathrm{~cm}^{-1}$. All data were processed using the Spectrum (PerkinElmer) software package and SpectraGryph 1.30.

Copolyamide films were prepared by dissolving around $1 \mathrm{~g}$ of a polymer in $10 \mathrm{~mL}$ HFIP. Upon full dissolution the mixture was placed in a teflon crystallization dish and left overnight partially covered under the fumehood in order to allow gradual evaporation of HFIP resulting in a thin polyamide film.

The microscopic images were recorded using an Olympus BX53 transmitted light microscope with cross polarizers equipped with a DP26 5 megapixel digital camera and a Linkam hotstage HFSX350. Around $50 \mathrm{mg}$ of salt mixture was place on the hot-stage and heated above the melting point of the salt. Data acquisition was performed using the Stream Essential software.

The biobased content (wt\%) was calculated according to the formula $\sum\left(\mathrm{n}_{\mathrm{bb}} * \mathrm{M}_{\mathrm{bb}}\right) / \sum\left(\mathrm{n}_{\mathrm{all}} * \mathrm{M}_{\mathrm{all}}\right)$ with $\mathrm{n}_{\mathrm{bb}}=$ mol content of biobased components, $\mathrm{M}_{\mathrm{bb}}=$ molecular weight of biobased componets $(\mathrm{g} / \mathrm{mol}), \mathrm{n}_{\text {all }}=$ mol content of each component and $\mathrm{M}_{\text {all }}=$ molecular weight of each component $(\mathrm{g} / \mathrm{mol})$ 


\section{REFERENCES}

1. S. N. Vouyiouka, E. K. Karakatsani and C. D. Papaspyrides, Prog. Polym. Sci., 2005, 30, 1037.

2. C. Lavilla, E. Gubbels, A. Martínez de Ilarduya, B. A. J. Noordover, C. E. Koning and S. Muñoz-Guerra, Macromolecules, 2013, 46, 4335-4345.

3. J. Zhao, H. Xu, J. Fang and J. Yin, J. Appl. Polym. Sci., 2012, 126, 244-252.

4. J. Thanuja and M. Srinivasan, Eur. Polym. J., 1988, 24, 1123-1126.

5. M. Ueda and S. Komatsu, J. Polym. Sci., Part A: Polym. Chem., 1989, 27, 1017-1026.

6. F. C. Chen, R. G. Griskey and G. H. Beyer, AIChE Journal, 1969, 15, 680-685.

7. I. Steinborn-Rogulska and G. Rokicki, Polimery, 2013, 58, 1-12.

8. A. Wroblewska, A. Zych, S. Thiyagarajan, D. Dudenko, D. van Es, M. R. Hansen, C. Koning, R. Duchateau and L. Jasinska-Walc, Polym. Chem., 2015, 6, 4133-4143.

9. L. Jasinska, M. Villani, J. Wu, D. van Es, E. Klop, S. Rastogi and C. E. Koning, Macromolecules, 2011, 44, 3458-3466.

10. E. Gubbels, C. Lavilla, A. M. de Ilarduya, B. A. J. Noordover, C. E. Koning and S. MuñozGuerra, J. Polym. Sci., Part A: Polym. Chem., 2014, 52, 164-177.

11. R. Sablong, R. Duchateau, R. Koelewijn, C. E. Koning, G. de Wit and J. van Haveren, Biomacromolecules 2008, 9, 3090-3097.

12. S. Cakir, M. Nieuwenhuizen, P. G. A. Janssen, R. Rulkens and C. E. Koning, Polymer, 2012, 53, 5242-5250.

13. C. Lavilla and S. Muñoz-Guerra, Green Chem., 2013, 15, 144-151.

14. J. Wu, L. Jasinska-Walc, D. Dudenko, A. Rozanski, M. R. Hansen, D. van Es and C. E. Koning, Macromolecules, 2012, 45, 9333-9346.

15. C. Lavilla, A. Alla, A. M. de Ilarduya, E. Benito, M. G. Garcia-Martin, J. A. Galbis and S. Munoz-Guerra, Biomacromolecules, 2011, 12, 2642-2652.

16. C. Lavilla, A. Alla, A. Martínez de Ilarduya, E. Benito, M. G. García-Martín, J. A. Galbis and S. Muñoz-Guerra, J. Polym. Sci., Part A: Polym. Chem., 2012, 50, 1591-1604.

17. J. Kim, J. M. Park, H. S. Lee and E. Yohgana, US 2014/0228523 A1, 2014.

18. Y. K. Endah, S. H. Han, J. H. Kim, N.-K. Kim, W. N. Kim, H.-S. Lee and H. Lee, J. Appl. Polym. Sci., 2016, 133, n/a-n/a.

19. M. Cao, C. Zhang, B. He, M. Huang and S. Jiang, Macromol. Res., 2017, 25, 722-729.

20. N. Ogata, K. Sanui, T. Ohtake and H. Nakamura, Polym. J., 1979, 11, 827-833.

21. N. Ogata, K. Sanui, K. Hosoda and H. Nakamura, J. Polym. Sci., Polym. Chem. Ed., 1976, 14, 783-792.

22. F. Higashi, S. I. Ogata and Y. Aoki, J. Polym. Sci., Polym. Chem. Ed., 1982, 20, 2081-2087.

23. N. Ogata, K. Sanui, K. Hosoda and H. Nakamura, J. Polym. Sci., Polym. Chem. Ed., 1977, 15, 1523-1526.

24. N. Ogata, K. Sanui, H. Nakamura and H. Kishi, J. Polym. Sci., Polym. Chem. Ed., 1980, 18, 933-938.

25. M. E. Rogers and T. E. Long, Synthetic methods in step-growth polymers., A John Wiley \& Sons, inc., Hoboken, New Jersey, 2003.

26. J. A. Galbis, M. de Gracia Garcia-Martin, M. V. de Paz and E. Galbis, Chem. Rev., 2016, 116, 1600-1636.

27. S. Munoz-Guerra, High Perform. Polym., 2012, 24, 9-23.

28. W. A. P. Black, E. T. Dewar and D. Rutherford, U.S. Patent 3,225,012, 1965.

29. T. P. Bird, W. A. P. Black, E. T. Dewar and J. B. Hare, J. Chem. Soc., 1963, 0, 3389-3391.

30. T. P. Bird, W. A. P. Black, E. T. Dewar and H. W. T. Rintoul, J. Chem. Soc., 1964, 0, 45124521.

31. F. Fenouillot, A. Rousseau, G. Colomines, R. Saint-Loup and J. P. Pascault, Prog. Polym. Sci., 2010, 35, 578-622.

32. S. Thiyagarajan, L. Gootjes, W. Vogelzang, J. van Haveren, M. Lutz and D. S. van Es, ChemSusChem, 2011, 4, 1823-1829.

33. M. Metzke and Z. Guan, Biomacromolecules, 2008, 9, 208-215. 
34. V. Froidevaux, C. Negrell, S. Caillol, J. P. Pascault and B. Boutevin, Chem. Rev., 2016, 116, 14181-14224.

35. C. Stevens and R. Verhé, Renewable Bioresources: Scope and Modification for Non-Food Applications, John Wiley \& Sons, West Sussex, 2004.

36. K. D. Green, M. K. Turner and J. M. Woodley, Enzyme Microb. Technol., 2000, 27, 205-211.

37. J. Tomaszewska, D. Bielinski, M. Binczarski, J. Berlowska, P. Dziugan, J. Piotrowski, A. Stanishevsky and I. A. Witonska, RSC Advances, 2018, 8, 3161-3177.

38. A. A. Wróblewska, S. M. A. De Wildeman and K. V. Bernaerts, Polym. Degrad. Stab., 2018, 151, 114-125.

39. C. Lavilla, A. Alla, A. Martínez de Ilarduya, E. Benito, M. G. García-Martín, J. A. Galbis and

S. Muñoz-Guerra, J. Polym. Sci., Part A: Polym. Chem., 2012, 50, 3393-3406.

40. C. Lavilla and S. Muñoz-Guerra, Polym. Degrad. Stab., 2012, 97, 1762-1771.

41. C. Lavilla, A. Alla, A. Martínez de Ilarduya and S. Muñoz-Guerra, Biomacromolecules, 2013, 14, 781-793.

42. T. W. Greene and P. G. M. Wuts, Greene's protective groups in organic synthesis., John Wiley \& Sons, Inc., Hoboken, New Jersey, 2007.

43. C. G. Sauer, I. Barnes, K. H. Becker, H. Geiger, T. J. Wallington, L. K. Christensen, J. Platz and O. J. Nielsen, J. Phys. Chem. A, 1999, 103, 5959-5966.

44. W. B. Guenther and W. D. Walters, J. Am. Chem. Soc., 1951, 73, 2127-2131.

45. A. A. Wróblewska, K. V. Bernaerts and S. M. A. De Wildeman, Polymer, 2017, 124, 252262.

46. S. Huijser, G. D. Mooiweer, R. van der Hofstad, B. B. P. Staal, J. Feenstra, A. M. van Herk, C. E. Koning and R. Duchateau, Macromolecules, 2012, 45, 4500-4510.

47. S. Huijser, B. B. P. Staal, J. Huang, R. Duchateau and C. E. Koning, Biomacromolecules 2006, 7, 2465-2469. 



\section{Chapter 5 THERMAL STABILITY OF GALX DURING POLYMERIZATION}

Abstract

This chapter focuses on the polymerizability of GalX acids and esters and on the identification of side reactions during polymerizations in different conditions. Factors like the general availability of carbohydrate sources and the unique properties of polymers therefrom contribute to their popularity, but on the other hand the presence of additional functional groups e.g. cyclic acetal, might lead to side reactions. The substrates and the polymers obtained by means of the melt polycondensation of ester functionalized monomers and polyamide salts are analyzed by combined TGA, GPC, NMR, LC-MS and Maldi-ToF techniques. Furthermore, melt polymerization of polyamide salts was followed by TGA-MS, which allowed identification of the degradation products produced during the polymerization. The i-propylidene acetal is more sensitive to acidic hydrolysis than the methylene acetal, so it should not be used in the free acid form to avoid internally-induced hydrolysis. The methylene acetal is more stable in the acidic conditions but undergoes more readily temperature-induced radical degradation than the i-propylidene acetal. This different behavior can be explained because the i-propylidene acetal forms stable intermediates during cationic degradation (tertiary carbocation) and the methylene acetal possess multiple sides on which a radical intermediate can be formed due to the extraction of hydrogen. 



\subsection{Introduction}

Unique cyclic moieties of different carbohydrate-derived compounds are known to enhance the thermal properties of polymers ${ }^{1}$; however the presence of oxygen linkages may hamper the thermal stability of those moieties during or after polymerization, and therefore lead to the occurrence of side reactions, manifested in discoloration, crosslinking or degradation of the material ${ }^{2}$. This phenomenon is particularly disturbing during high temperature polycondensation required for the synthesis of polyamides. ${ }^{3}$ The identification of the degradation mechanism might be a key factor to limit side reactions occurring during polymerization.

There have been a significant number of papers released regarding side reactions during polyamide synthesis. For example, Flanningan et al. ${ }^{4}$ reported the ethylation of amine groups by the ester group of diester functional monomer during ester-amide interchange melt polycondensation. The research of Hellmann et al. ${ }^{5}$ and Malluche et al. ${ }^{6}$ confirmed Flanningan's findings and further investigated the kinetics of those side reactions occurring during both melt polycondensation of dicarboxylic acids and melt polycondensation of diesters with diamines. ${ }^{6,7}$

Simultaneously, the hydrolytic degradation processes of carbohydrate-derived compounds have been investigated. The group of Muñoz-Guerra ${ }^{8-11}$ prepared complex research regarding the biodegradability and hydrolytic degradation of (co)polyesters prepared from biacetalized alditols and aldarates. Methylene acetal moieties in the polymer backbone are stable during hydrolysis in acidic environment, while the ester linkages are cleaved. While methylene acetal units (e.g. GalXH) are stable in acidic conditions, isopropylidene acetal units (e.g. $\mathrm{GalXMe}$ ) deprotect easier by acidic hydrolysis in acids like formic acid or trifluoroacetic acid. $^{12,13}$

The different behavior of the two acetal derivatives during polymerization, described in Chapter 2 and Chapter 4, might be associated with their difference in reactivity. Ogata et al. ${ }^{14}$ revealed that different substituents on the $\alpha$ or $\beta$ carbon of the carboxylic acid significantly influence the reactivity of active esters of dicarboxylic acid during solution polymerization.

It is suspected that during the polymerization the acetal protection might be removed, leaving galactaric acid with free hydroxyl groups in the reaction. As hypothesized in previous 


\section{Chapter 5}

chapters, this results in multifunctional molecules that act as branching agent/crosslinker. Furthermore, as shown by Kiely et al. in the research investigating the behavior of unprotected galactaric acid and polyamides therefrom, those unprotected hydroxyl species contribute to the formation of cyclic lactones during the synthesis. ${ }^{15-17}$ Additionally, numerous papers have been released describing the polymerization of monoacetalized tartaric acid $^{18-21}$ in which the formation of cyclic 5 member ring imides from amine and tartarates was discovered. ${ }^{18}$

The present study focuses on the different degradation processes which occur during the melt polycondensation of biacetalized galactaric aicd derivatives with diamines. Both melt polycondensation of dicarboxylic acid GalX salts and melt polycondensation of GalX diethyl esters, will be investigated. A key aspect of the presented study is the degradation of acetal moieties during polymerization and after it is incorporated into the polymer backbone, both thoroughly investigated by the combined GPC, NMR, TGA-MS, LC-MS and Maldi-ToF techniques. Among the complicated mixture of side products, which are formed during polymerization, the most important ones were identified and are presented in this chapter.

Based on the chemistry of acetals, a scheme (see Figure 5-1) was developed which presents potential chemical side reactions happening during the polymerization of GalX monomers. 


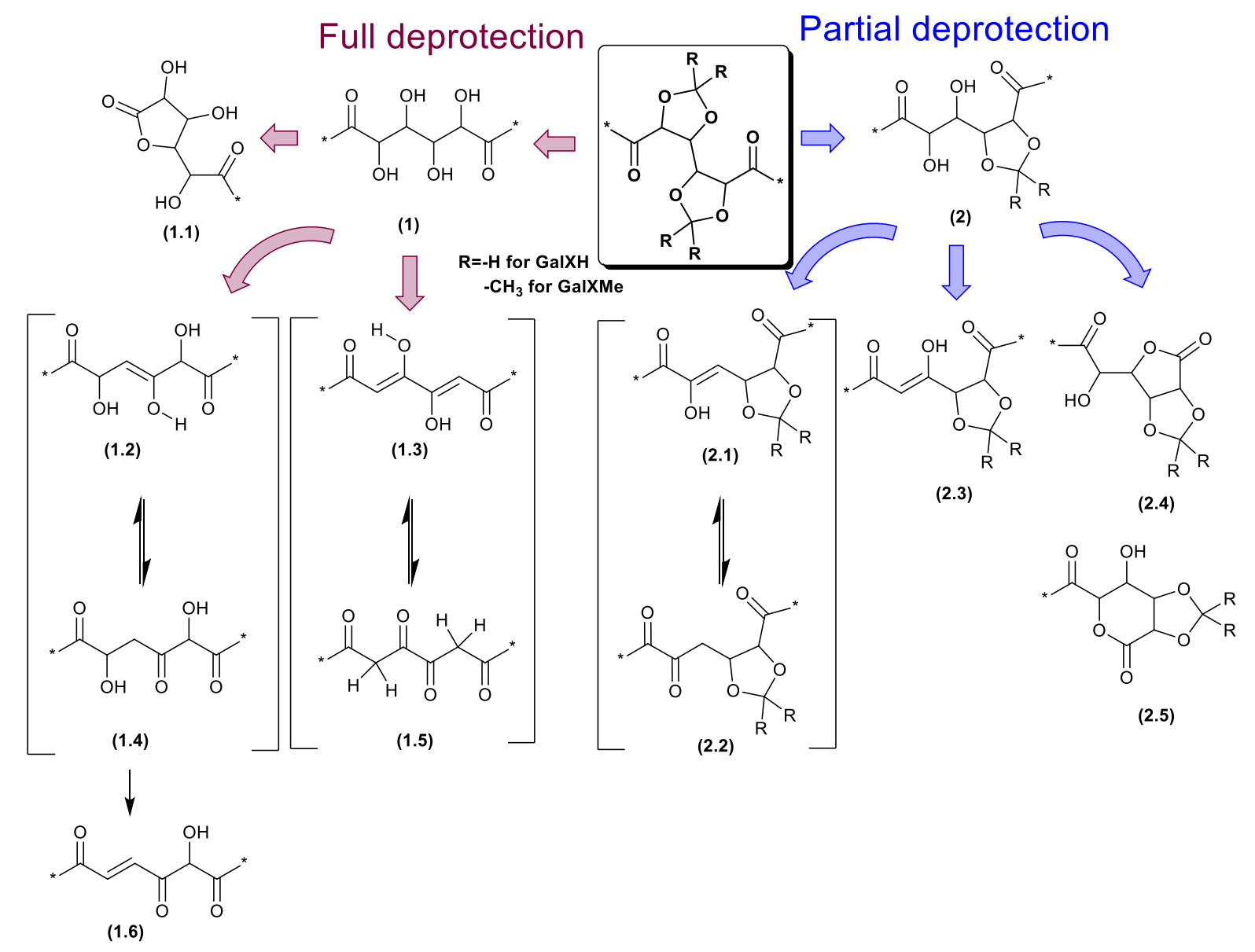

Figure 5-1 Possible side reactions occurring during high temperature melt polycondensation of GalX.

During high temperature processes like polycondensation of GalX several side reactions can occur. The (partial) deprotection of the GalX acetal leads to multifunctional monomers, which can cause branching and crosslinking during polymerization. The GalX molecule can be deprotected on one side (pathway 2, Figure 5-1) or on both sides (pathway 1, Figure 5-1). The free hydroxyl groups, which are present in the molecule upon the deprotection, can undergo dehydratation (1.2, 1.3, 1.6, 2.1 and 2.3 in Figure 5-1) or cyclization (1.1, 2.4 and 2.5 in Figure 5-1). The close vicinity of a hydroxyl group and a double bond is known to stimulate hydrogen transfer causing keto-enol tautomerization $(1.4,1.5$ and 2.2 in Figure 5$1)$.

\subsection{Synthesis of polyamides}

In order to investigate side reactions and predict possible degradation mechanisms during the formation of GalX polyamides two different approaches towards the synthesis of homopolyamides were chosen. The polymerization of polyamide (PA) salts is a preferable, industrially relevant reaction whose chemical pathway consists of a two-step process (Figure 


\section{Chapter 5}

5-2/I). First, the salt is synthesized, separated and purified and then it becomes a substrate in melt polycondensation. The choice of this method provides an equimolar ratio of reactive groups during polymerization, though due to the strong hydrogen bonding 22 the obtained salts are very often high melting compounds which might be a limiting factor for the application of this method to make homopolyamides from biobased GalX or other similar monomers. Salts of galactaric acid acetals with HMDA have melting temperatures of $261^{\circ} \mathrm{C}$ and $272^{\circ} \mathrm{C}$, for GalXMe and GalXH, respectively. The other approach is the synthesis of PAs from ethyl esters of carboxylic acids, which allows to conduct the synthesis at lower temperatures but at the same time does not prevent other side reactions like the (di)ethylation of amines to occur (Figure 5-2/II). ${ }^{7}$ The obtained polyamides show high discoloration which does occur already at an early stage of the synthesis.

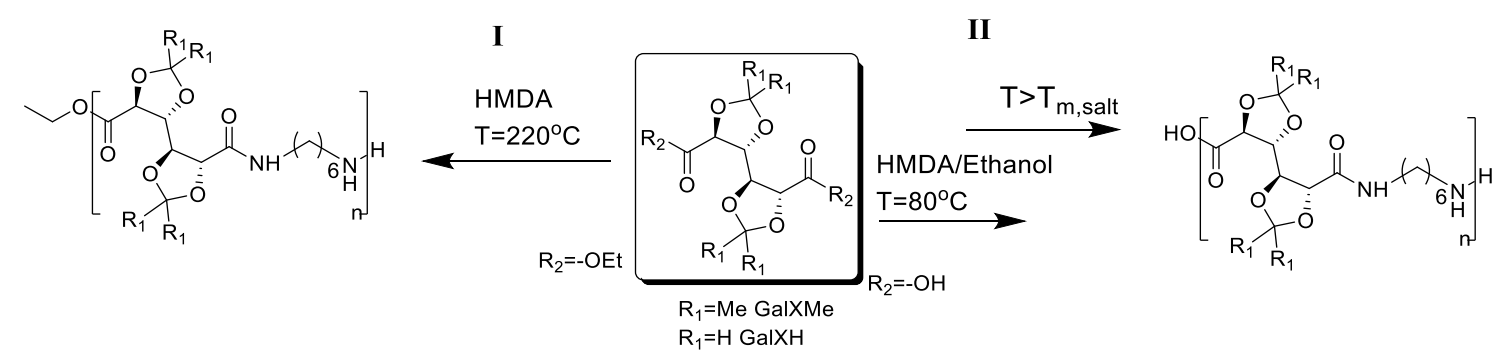

Figure 5-2 Two chemical pathways for PA synthesis: I Melt polymerization of polyamide salts II Melt polymerization of diethyl esters.

\subsubsection{Understanding of the processes occurring during salt polymerization}

The synthesis of polyamides from polyamide salts (Figure 5-2/I) at reaction temperatures above the melting point of the salts (resp. $261{ }^{\circ} \mathrm{C}$ for HMDA,GalXMe and $272{ }^{\circ} \mathrm{C}$ for HMDA,GalXH salts) results in fully degraded, black product. To understand the nature of this observed degradation, thermogravimetric analysis (TGA) coupled with mass spectrometry (MS) was performed. The SA-HMDA reference salt melts around $180{ }^{\circ} \mathrm{C}$ and starts to lose weight from $160{ }^{\circ} \mathrm{C}$ on (Figure 5-3). All polyamide salts were carefully dried before the TGA-MS measurement which suggests that the weight loss corresponds to the loss of water originating from the formation of the amide bond. At the bottom of the graph in Figure 5-3, the detector response of MS is presented with an indication which fragments were formed: water $\left[18 \mathrm{~g} \cdot \mathrm{mol}^{-1}\right]$, ammonia $\left[17 \mathrm{~g} \cdot \mathrm{mol}^{-1}\right]$, methane $\left[16 \mathrm{~g} \cdot \mathrm{mol}^{-1}\right]$, formaldehyde [29 $\left.\mathrm{g} \cdot \mathrm{mol}^{-1}\right]$, formic acid $\left[45 \mathrm{~g} \cdot \mathrm{mol}^{-1}\right]$, carbon dioxide $\left[44 \mathrm{~g} \cdot \mathrm{mol}^{-1}\right]$ and acetone $\left[59 \mathrm{~g} \cdot \mathrm{mol}^{-1}\right]$. 


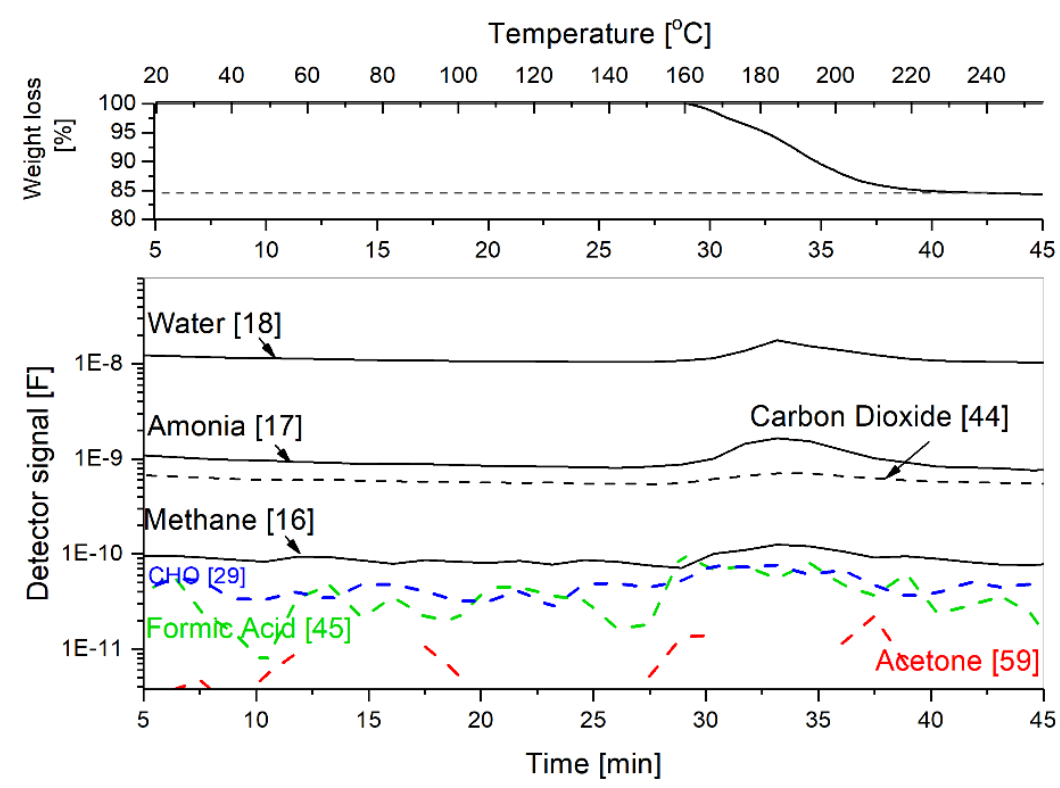

Figure 5-3 Thermogravimetric analysis (top) - mass spectrometry (bottom) results for heating the sebacic acid - 1,6-hexamethylenediamine (SA-HMDA) salt to $250{ }^{\circ} \mathrm{C}$ with a heating rate of $10{ }^{\circ} \mathrm{C} /$ min. Numbers in the brackets $[x]$ correspond to the $\mathrm{m} / \mathrm{z}$ values (in $\mathrm{g} \cdot \mathrm{mol}^{-1}$ ) used for identification of the corresponding fragments.

The two main fragments which were detected upon heating of the aliphatic salt are water and ammonia. A low intensity detector response was also registered for methane and carbon dioxide fragments. Those readings are treated as a reference for the degradation study of GalX salts. The interpretation of side reactions during degradation of SA-HMDA salt is not in the scope of this study.

a.

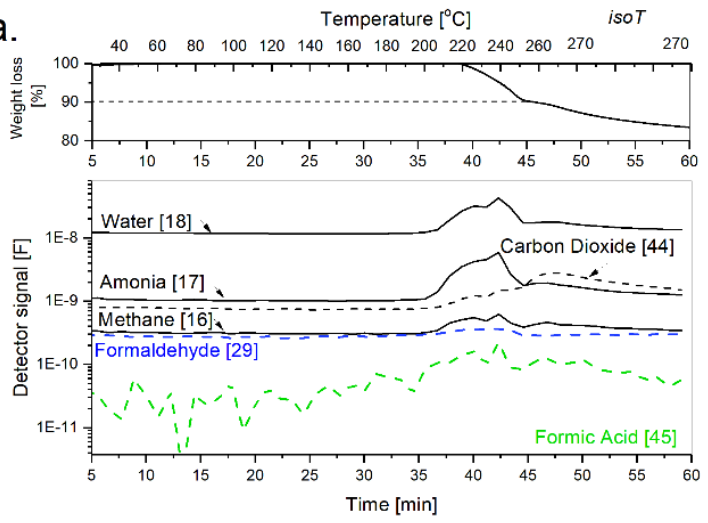

b.
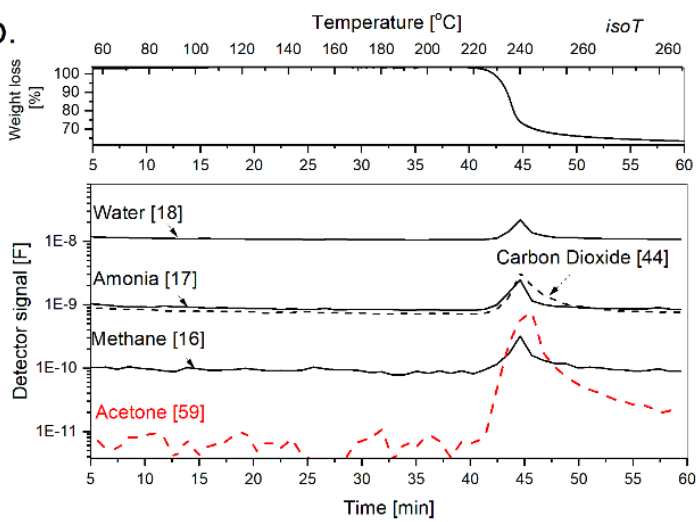

Figure 5-4 Thermogravimetric analysis (top) - mass spectrometry (bottom) results for heating GalX - HMDA salts. (a) GalXH-HMDA salt heated to $270{ }^{\circ} \mathrm{C}$ with a heating rate of $10^{\circ} \mathrm{C} / \mathrm{min}$ followed by an isothermal program at $270{ }^{\circ} \mathrm{C}$. (b) GalXMe-HMDA salt heated to $260^{\circ} \mathrm{C}$ with a heating rate of $10^{\circ} \mathrm{C} / \mathrm{min}$ followed by an isothermal program at $260^{\circ} \mathrm{C}$. Numbers in the brackets $[x]$ correspond to the $\mathrm{m} / \mathrm{z}$ values (in $\mathrm{g} \cdot \mathrm{mol}^{-1}$ ) used for identification of corresponding fragments.

The thermal degradation profiles of GalXH and GalXMe salts displayed in Figure 5-4 prove different breakdown of the GalX molecule upon heating than for the aliphatic reference salt. 


\section{Chapter 5}

When the measurement cell approaches the melting points of the GalX salt a rapid weight decrease is registered (Figure 5-4/top), which after 60 minutes of the experiment is significantly steeper for GalXMe than for GalXH. The abovementioned weight loss is a result of both polymerization processes and most importantly, degradation reactions. The degradation profile of the GalXMe salt (Figure 5-4 (a)) shows, except the reference response also registered for aliphatic salts, an intensive signal for the acetone related to the acetal deprotection. The profile of GalXH (Figure 5-4 (b)), on the other hand, consists of two parts with different weight loss rates. In the first step above $220{ }^{\circ} \mathrm{C}$ (still in the solid state) water, ammonia and methane fragments are registered as strong signals and formaldehyde, carbon dioxide and formic acid as weaker responses. In the second step above $250{ }^{\circ} \mathrm{C}$ the signal of carbon dioxide increases. Carbon dioxide gives very low intensity peak on the reference scan of the SA-HMDA salt and therefore it is believed that the increased carbon dioxide peak in GalXH-HMDA and GalXMe-HMDA corresponds to thermal oxidation of products which are released during acetal dissolution (acetone, formaldehyde). It is also highly possible that the different reactivity of the carboxyl group of both GalX species in comparison to aliphatic diacids is stimulating faster decarboxylation of those groups at elevated temperatures, releasing carbon dioxide. The mentioned degradation processes are summarized in Figure 55.
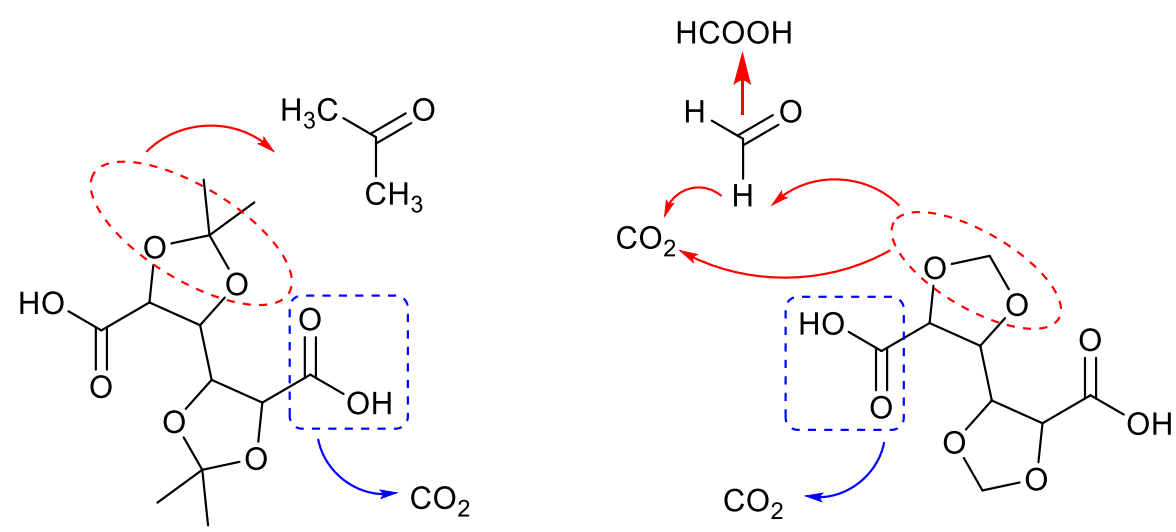

Figure 5-5 Possible degradation pathways of GalXMe and GalXH dicarboxylic acids during polyamide salt polymerization.

Both GalX salts are degrading rapidly upon heating, however those degradation processes are observed mostly around the melting point of salts, and therefore it is concluded that the crystalline network of the organic salts acts as a protection for the acetal. Some degradation/polymerization related weight loss for GalXH and GalXMe is already observed below the melting point. When molecules become mobile at high temperature, degradation processes are rapid and result in the deprotection of acetal groups and decarboxylation of the 
acid groups. During degradation carbon dioxide and acetone are formed from GalXH and GalXMe acetal moieties, respectively (Figure 5-5). It is highly probable that the resulting hydroxyl groups are not stable at those elevated temperatures and their presence leads to further degradation of the compounds (see proposed reaction routes in Figure 5-1).

\subsubsection{Polymerization via ester-amide exchange}

Since the synthesis of GalX polyamides via the salt route was unsuccessful, melt polycondensation of diethylesters and diamines was investigated as an alternative (Figure 52/II). GalX diethylesters have significantly lower melting points (GalXMe diethyl ester $87^{\circ} \mathrm{C}$, GalXH diethyl ester $110{ }^{\circ} \mathrm{C}$ ) than the corresponding polyamide salts and require lower temperatures during the synthesis. All ingredients are already molten above $100{ }^{\circ} \mathrm{C}$ and therefore the reaction mixture can be stepwise heated in the molten state to the required reaction temperature. During the stepwise heating the formation of oligomers which are thermally more stable than the monomers begins at lower temperatures. Despite this, side reactions could not be completely eliminated as manifested by polymer discoloration and increased dispersities of products during polymerization. ${ }^{13}$

In order to identify which side products are present in the polymer, model systems were prepared and investigated for both GalXH and GalXMe, namely BnA-GalX-BnA diamides $(\mathrm{BnA}=$ benzylamine) (Figure 5-6). While the polymers described in this chapter have HMDA as diamine, a benzylamine diamide was made because it allows straightforward analysis of NMR spectra.

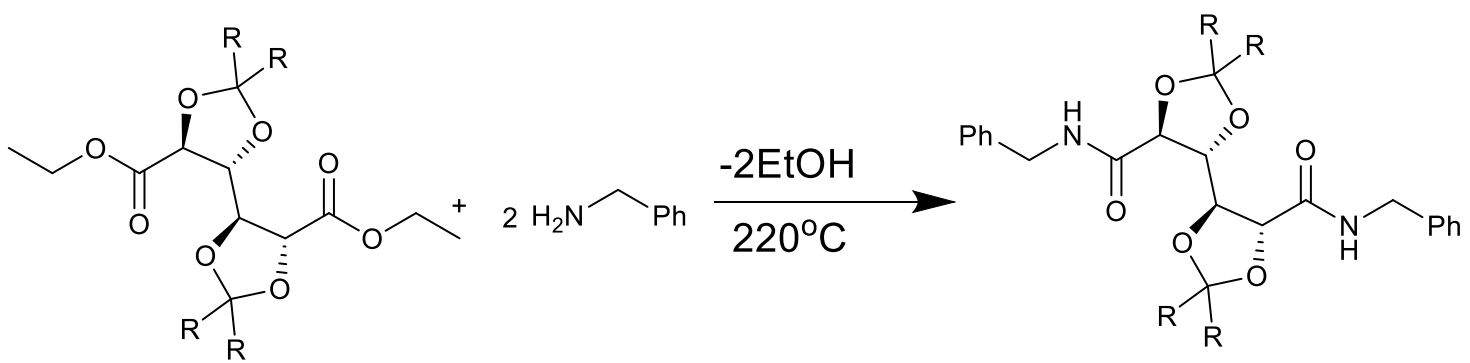

GalX
BnA
BnA-GalX-BnA

$\mathrm{R}=\mathrm{H}, \mathrm{BnA}-\mathrm{GalXH}-\mathrm{BnA}$ $\mathrm{R}=\mathrm{CH}_{3}, \mathrm{BnA}-\mathrm{GalXMe-BnA}$

Figure 5-6 Synthesis of GalX diamides for model studies. 


\section{Chapter 5}

a) Thermal treatment of diamides and analysis of the degradation products

From literature it is known that during melt polycondensation polyamides can crosslink which is attributed to multiple reactions of amine hydrogens leading to condensation of amines. ${ }^{3,7}$ Besides this, based on our previous results and based on the results of TGA-MS it is hypothesized that (partial) degradation of the acetal group of GalX during polycondensation gives rise to reactive sides on the backbone (hydroxyl groups, double bonds) and therefore further contributes to branching and/or crosslinking. In order to confirm the correctness of the hypothesis, purified BnA-GalX-BnA diamide model compounds were subjected to polymerization conditions $\left(220^{\circ} \mathrm{C}\right.$ and higher) and the resulting (partially degraded) products were investigated using GPC and NMR. The temperature exposure began at $220^{\circ} \mathrm{C}$ and the temperature was gradually raised to $280{ }^{\circ} \mathrm{C}$, at which temperature the diamides were held for 9 hours. Upon exposure of the sample to $220^{\circ} \mathrm{C}$ and $250{ }^{\circ} \mathrm{C}$ almost no discoloration was observed. The results were compared with pure, non-degraded species.

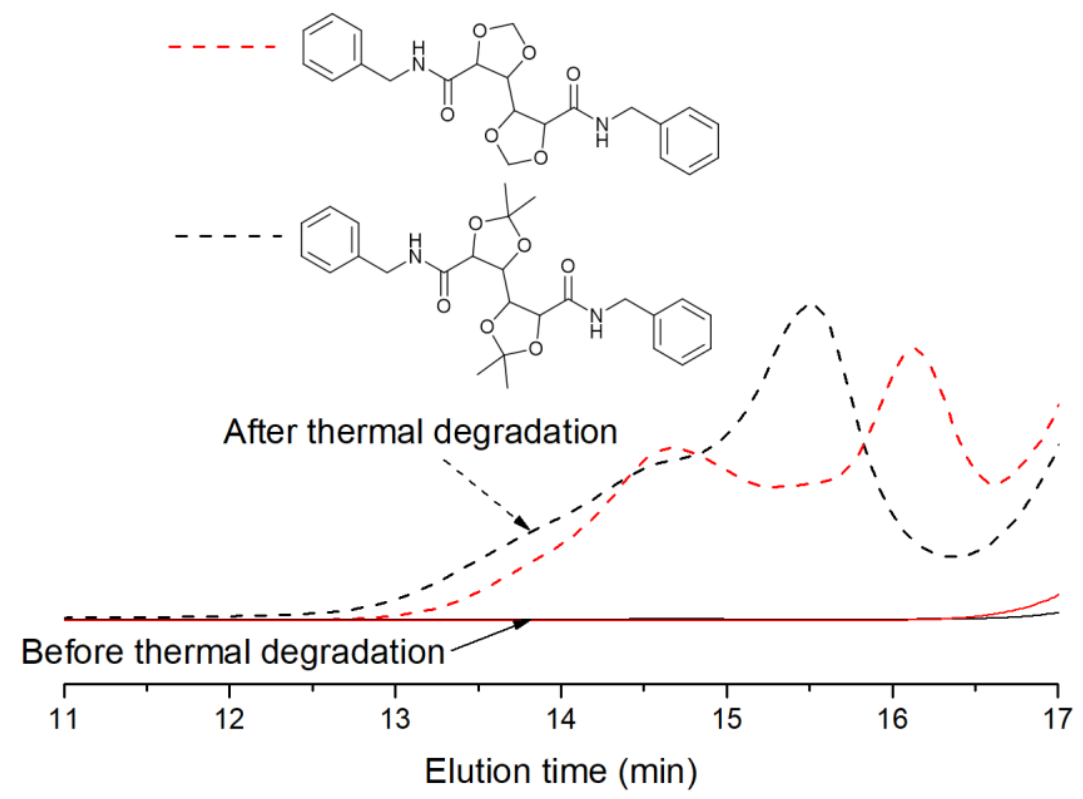

Figure 5-7 GPC traces of GalXH and GalXMe diamide model compounds before and after 15 degradation test $\left(3\right.$ h at $220^{\circ} \mathrm{C}, 3 \mathrm{~h}$ at $250^{\circ} \mathrm{C}$ and $9 \mathrm{~h}$ at $\left.280^{\circ} \mathrm{C}\right)$.

The appearance of a shoulder on the GPC chromatogram (Figure 5-7) upon heating of the diamide model compound can be correlated with the formation of a high molecular weight fraction. During polyamide synthesis the formation of a high molecular weight shoulder can be caused by branching/crosslinking due to the condensation of diamines or by the decay of the acetal. In the model system with diamides no free diamine is present; however during thermal degradation of the amide bond some amine might be released. It is believed that free 
hydroxyl groups can directly react with a diamine to form secondary amine, condense with each other or undergo dehydration with the formation of double bonds.

Subsequently, the diamide model compounds, which were exposed to high temperature for elongated time period (15 h), were analyzed by ${ }^{1} \mathrm{H}$ NMR and ${ }^{13} \mathrm{C}$ NMR/DEPT-135, to see which regions of the molecules are the most susceptible to degradation (Figure 5-8). After the test both diamide model compounds had brown-black colors but were fully soluble in DMSO.

a.
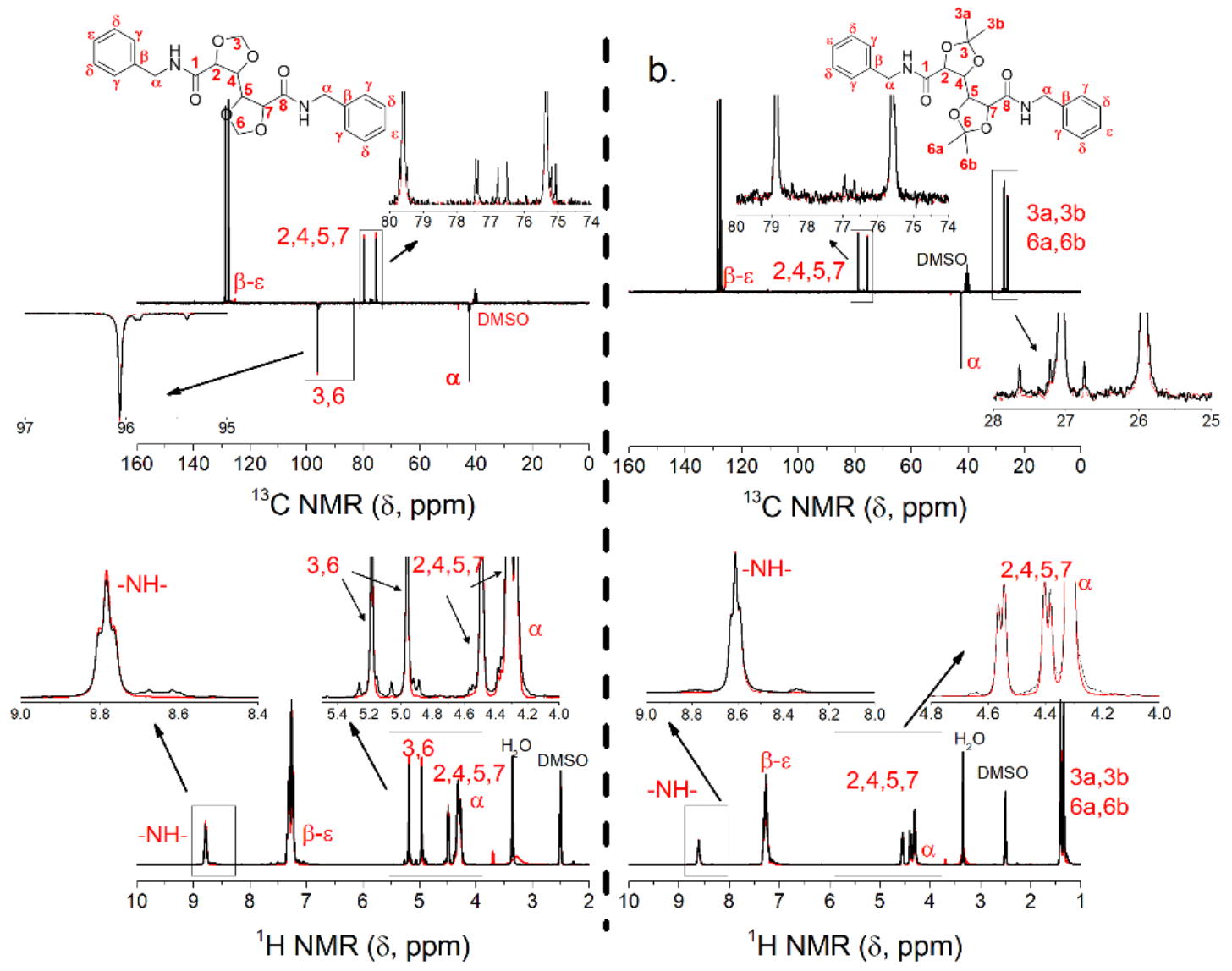

Figure 5-8 ${ }^{1} H$ NMR (bottom) and ${ }^{13} C N M R / D E P T$ (top) of two diamide model compounds before (red line) and after (black line) the thermal exposure program. (a) BnA-GalXH-BnA diamide and (b) BnA-GalXMe-BnA diamide. Regions: region of amide protons $-\mathrm{NH}$-, region corresponding to acetal protection (3, 6 for GalXH or $3 a, 3 b, 6 a, 6 b$ for GalXMe), region corresponding to GalX backbone (2, 4, 5, 7). Spectra after 15 h degradation test $\left(3 \mathrm{~h}\right.$ at $220^{\circ} \mathrm{C}, 3 \mathrm{~h}$ at $250^{\circ} \mathrm{C}$ and $9 \mathrm{~h}$ at $\left.280^{\circ} \mathrm{C}\right)$. All spectra are normalized to the aromatic signals (at 7.3 ppm). ${ }^{13} \mathrm{C} \mathrm{NMR/DEPT:}-\mathrm{CH}_{3},-\mathrm{CH}$ - signals up, $\mathrm{CH}_{2}$ - signals down.

On both GalXH (Figure 5-8 (a)) and GalXMe (Figure 5-8 (b)) diamide model compound spectra, there are three general regions which are sensitive to elevated temperatures. First of all, changes are observed around the amide proton signals (8.6 ppm in $\left.{ }^{1} \mathrm{H} N \mathrm{NR}\right)$, which shows that during degradation the environment around the amide groups changes. For GalXH the appearance of two new signals was registered at 8.62 and $8.69 \mathrm{ppm}$, whereas for GalXMe 


\section{Chapter 5}

only one at $8.34 \mathrm{ppm}$. Both the intensities and the amount of side product peaks are lower for GalXMe than for GalXH on normalized NMR spectra.

A second region which is considered as an important region for analysis is the region of the GalX backbone protons, represented by the signals $2,4,5,7$, with a chemical shift between 4.00-4.65 ppm on ${ }^{1} \mathrm{H}$ NMR spectra and between 75.0-80.0 ppm on ${ }^{13} \mathrm{C}$ NMR spectra. On both ${ }^{1} \mathrm{H}$ NMR spectra a new peak is appearing at $4.55 \mathrm{ppm}$ (GalXH) or $4.65 \mathrm{ppm}$ (GalXMe). It is suspected that this peak corresponds to the proton located on backbone carbon with free hydroxyl groups instead of acetal (structures 1 and 2 in Figure 5-1). To support this, the interpretation of the ${ }^{13} \mathrm{C}$ NMR/DEPT signals suggests that carbons $(76-78 \mathrm{ppm})$ which are attached to those protons clearly are of the $-\mathrm{CH}$ type and it seems that the signals are generally shifted towards lower ppm values compared to not degraded diamide model compounds which correspond to a change of substituent from ether to hydroxyl. Again more side products are visible on the spectrum of GalXH than on the GalXMe spectrum.

The third region of interest is the region of the acetal protection itself. For GalXH it is represented by the region $4.80-5.30 \mathrm{ppm}\left({ }^{1} \mathrm{H} \mathrm{NMR}\right)$ and $95.50-96.50 \mathrm{ppm}\left({ }^{13} \mathrm{C}\right.$ NMR/DEPT). On both spectra new peaks are appearing, which correspond to $-\mathrm{CH}_{2}$ - groups. Similar changes are observed in the corresponding region for GalXMe diamide model compounds at $1.10-1.55 \mathrm{ppm}$ in the ${ }^{1} \mathrm{H}$ NMR spectrum and between $25-28 \mathrm{ppm}$ in ${ }^{13} \mathrm{C}$ $\mathrm{NMR}$, but in this case ${ }^{13} \mathrm{C}$ NMR signals belong to $-\mathrm{CH}_{3}$ groups.

The conclusion from these experiments is that the amount of side products appearing during exposition of the diamide model compounds to elevated temperatures, is not substantial. It was expected that in these conditions the spectrum would be less clear and would contain more signals to interpret. Additionally, those signals which have low intensity, still contribute to the high discoloration of the material. Analysis also reveals that thermal degradation processes are more common in case of GalXH than for GalXMe.

b) Analysis of side products formed during the synthesis of diamide model compounds.

To elucidate the chemical transformations that GalX diethyl ester molecules undergo during polymerization at elevated temperatures $\left(>220^{\circ} \mathrm{C}\right)$, a thorough investigation was done of the processes occurring during the synthesis of BnA-GalX-BnA diamide model compounds under reaction conditions similar as during polycondensation. The reaction mixtures after diamide model compound preparation were extracted with acetonitrile (ACN) and separated by HPLC with a mass spectrometry detector. Both diamides, BnA-GalXH-BnA and BnA- 
GalXMe-BnA, exhibit poor solubility in ACN and therefore due to the extraction procedure the concentration of all side products was increased in comparison to the concentration of the main product. The resulting chromatograms show multiple peaks with good separation (Figure 5-9). The scheme presented in Figure 5-1 was used as a basis to assign structures to the different molecular masses. The main identified side products are shown in Figure 5-10.
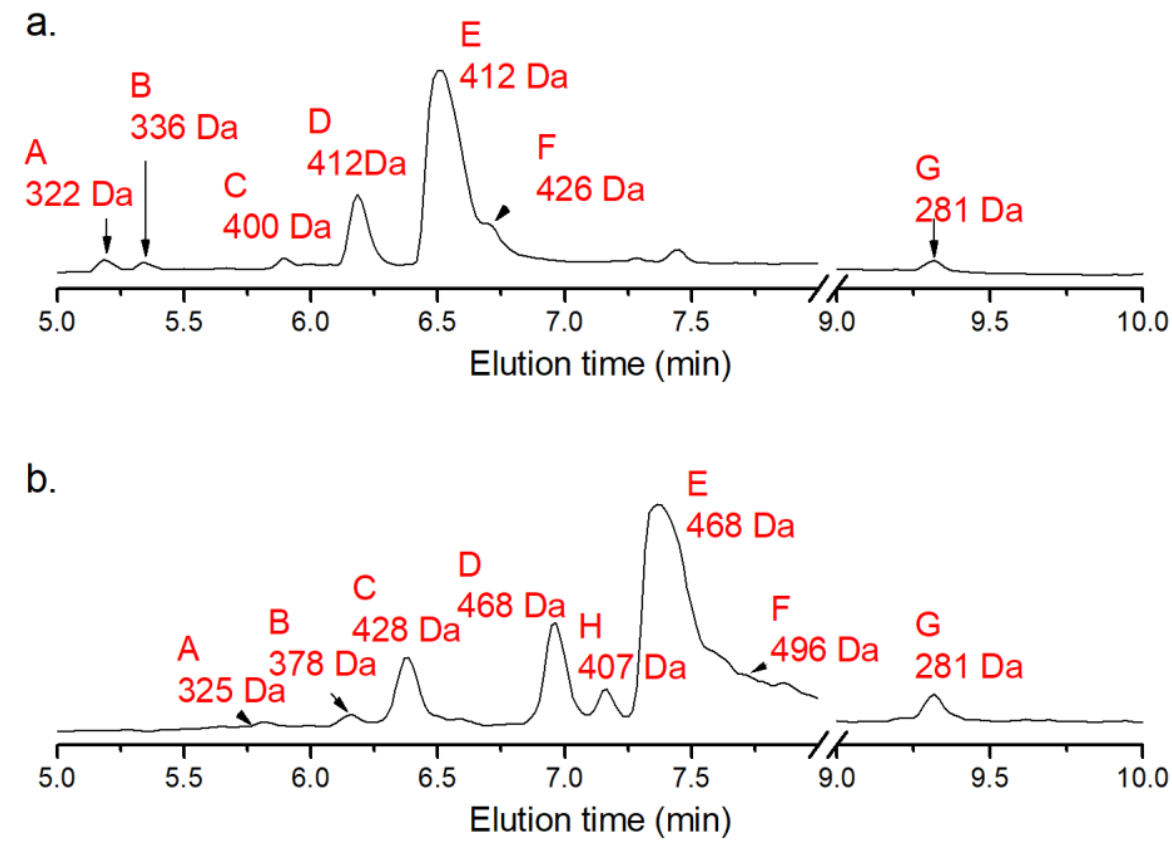

Figure 5-9 LC-MS chromatogram for positive charged ionization recorded for the reaction mixture during the formation of diamide model compounds (a) BnA-GalXMe-BnA, (b) BnA-GalXH-BnA.

It was earlier hypothesized that GalX molecules undergo deprotection at higher temperatures releasing volatile low molecular weight products (acetone, formic acid) resulting in multifunctional compounds which can significantly disrupt the synthesis. In fact, peak $\mathrm{C}$ in Figure 5-9 corresponds to partially deprotected GalX ((2), pathway 2, Figure 5-1). This peak is of significantly higher intensity for BnA-GalXMe-BnA than for BnA-GalXH-BnA, however the presented chromatograms should not be interpreted quantitively, since no data are available about extraction yields. Moreover, it is known that the two GalX derived compounds differ significantly in solubility. The fact that peaks corresponding to the desired diamide model compounds (peak E, 412 Da for BnA-GalXH-BnA and 468 Da for BnAGalXMe-BnA) can be identified implies that only partial cleavage of the acetal is occurring. Nonetheless, both pathways lead to the formation of additional functional groups, which undermines the sensitive balance between reactive groups (namely ethyl ester and amine groups) required during the polymerization (equimolarity). Additionally, it is suspected that 


\section{Chapter 5}

those partially cleaved fragments can easily form cyclic structures by the reaction between double bonds and amine groups (peak D, Figure 5-9 and Figure 5-10). The occurrence of double bonds is confirmed by the presence of the corresponding structures in mass and along with this partial cleavage might indicate that thermally driven radical decay processes are occurring. Dioxolane fragments were already proven to undergo radical degradation to double bonds with simultaneous release of formic acid and/or formaldehyde. ${ }^{23,24}$
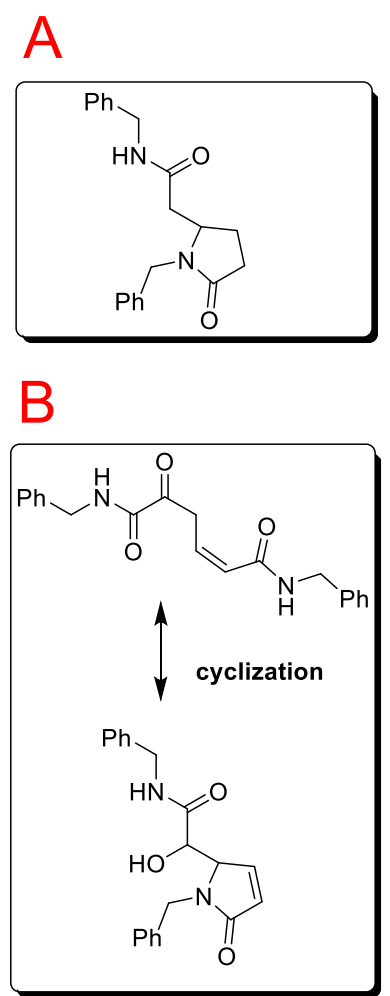

R-H, GalXH $\mathrm{R}-\mathrm{CH}_{3}, \mathrm{GaIXMe}$
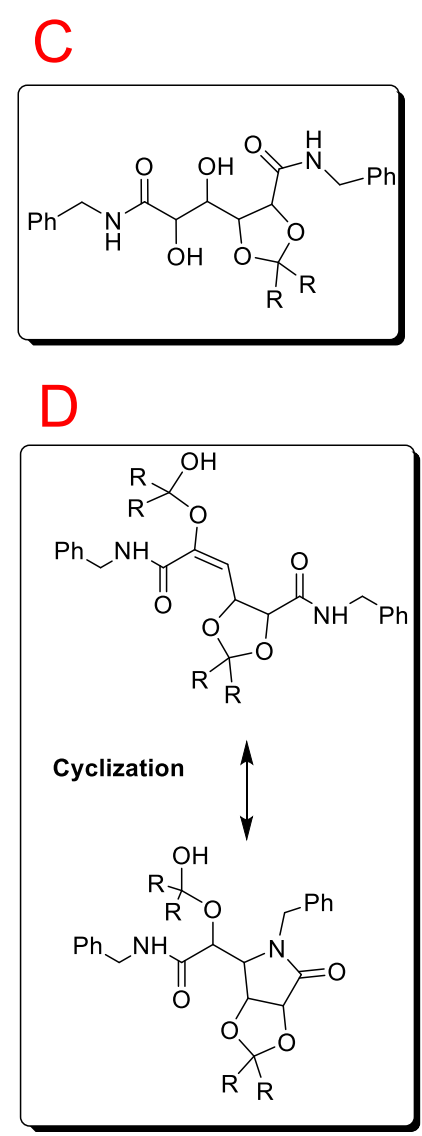

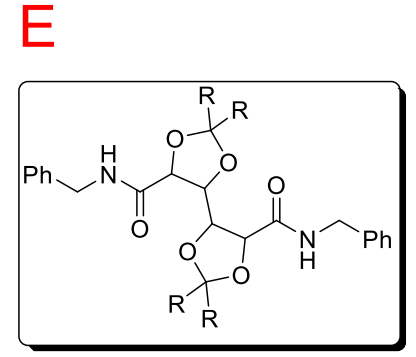

$\mathrm{F}$

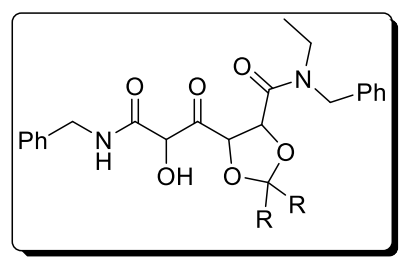

$\mathrm{G}$

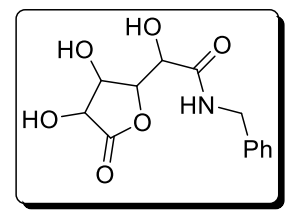

Figure 5-10 Possible degradation products formed during the diamide synthesis.

In spite of the fact that fully deprotected molecules (pathway 1, Figure 5-1) were not separated and identified, the products derived from that structure are represented by peak $\mathrm{G}$ on both chromatograms in Figure 5-9 (structure 1.1, Figure 5-1). This cyclic structure is a result of intramolecular transesterification of the deprotected molecule which results in a stable 5membered ring lactone. This behavior shows some similarities with lactone formation by glucaric acid in solution, which was thoroughly investigated by Brown et. al. ${ }^{15}$ The presence of cyclic species like $\mathrm{G}$ rather acts as a crosslinking agent than as a chain stopper.

Due to the slower kinetics of the reaction between amine and GalXMe compared to GalXH ${ }^{13}$, the reaction with GalXMe did not reach full conversion. Consequently, in the reaction 
mixture diamide with, on one side, ethyl ester groups and, on the other side, amide groups were identified (peak H in Figure 5-9 (b)). However, for the reaction between amine and GalXH, no traces of GalXH with ethyl ester are left.

\subsection{Polymerization reactions}

The results of the above described model study on diamides can be translated to the polymerization of GalX monomers. This means that fragmentation of the GalX molecule with the formation of multifunctional $\mathrm{OH}$ species, cyclic species and double bonds can occur during polymerization and contribute to the discoloration of the material as well as to destruction of the original equimolarity of the functional groups. The last phenomenon has according to the Carothers equation for polycondensation a big influence on the polymer structure (branching/crosslinking due to multifunctional species) and molecular weight (chain stopping), even if it happens only in small quantities.

In order to assess whether similar processes as observed for the model compounds are also observed during polymerization, GalX-HMDA polymers were prepared and analyzed via NMR, Maldi-ToF and GPC.

\subsubsection{NMR analysis of polymers.}

The NMR analysis was carried out on GalX-HMDA polymer samples which were prepared in small, closed vials at $220^{\circ} \mathrm{C}$ to ensure that volatile side products cannot leave the reaction system which allows their analysis. Typical NMR spectra can be found in Figure 5-11. 


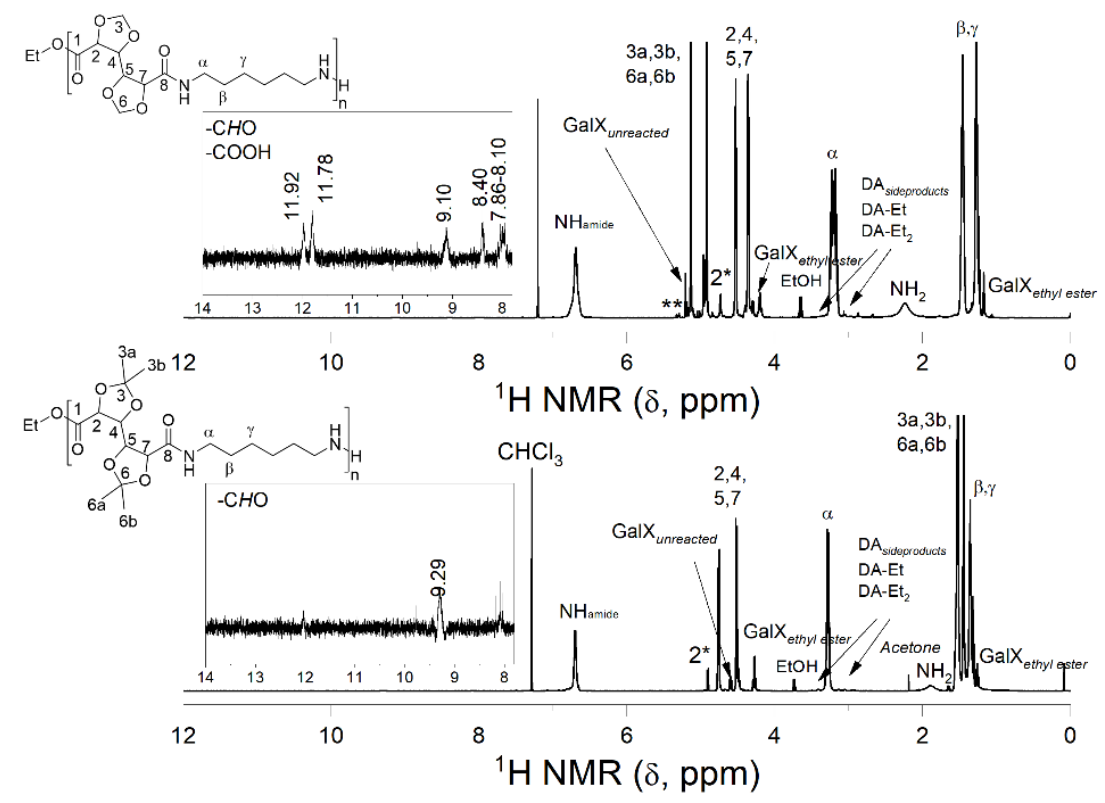

Figure 5-1 $1{ }^{1} \mathrm{H} \mathrm{NMR}\left(300 \mathrm{MHz}, \mathrm{CDCl}_{3}\right)$ of GalX-HMDA polymers after 90 minutes of reaction carried out in a closed vial. GalXH-HMDA (top) with $M_{n}=5100 \mathrm{~g} \cdot \mathrm{mol}^{-1}, D=3.46$ (GPC in HFIP) and GalXMe-HMDA (bottom) with $M_{n}=6500 \mathrm{~g} \cdot \mathrm{mol}^{-1}, D=2.54$ (GPC in HFIP). $2 *$ corresponds to the peak of GalX reacted on one side with the diamine, $D A-E t / D A-E t_{2}$ are peaks corresponding to ethylated and diethylated amine groups.

Figure 5-12 shows the evolution of the most important NMR signals as a function of time. GalX-HMDA polymerizations are characterized by a decrease of the GalX ethyl ester signal (4.28 ppm for GalXMe in Figure 5-12 (a) and 4.20 ppm for GalXH in Figure 5-12(c)) as a function of time what is expected upon reaction with diamine. The increase of peak $2 *$ at 4.74 ppm as a function of time was correlated with the formation of partially reacted GalX with only on one side diamine. Since during the polycondensation volatile products were not removed, the molecular weight of the polymer is low.

For the GalXMe-HMDA polymerization, an increase of the acetone signal is observed as a function of time (Figure 5-12 (a)), originating from partial deprotection of the acetal. On both GalXH (Figure 5-12 (b)), and GalXMe (Figure 5-12(a)) spectra additional proton signals appear above 7-8 ppm after 10-40 minutes reaction. For the GalXMe spectrum those are two signals at $8.10 \mathrm{ppm}$ and $9.29 \mathrm{ppm}$ (typical for aldehydes) and for GalXH multiple signals are visible between 7.90 - 12.00 ppm (typical for aldehydes and carboxylic acids). Those aldehyde and carboxylic acid species are likely to be GalX degradation products which result from acetal deprotection during polymerization conditions. As already mentioned in the TGA-MS section, the released volatile components can undergo further reactions like thermal oxidation (Figure 5-5). It is expected that formaldehyde released upon deprotection of GalXH readily undergoes further reactions which explains multiple peaks in the aldehyde and 
carboxylic acid region. Also the oxidation of alcohol groups (formed upon acetal deprotection) to aldehydes/carboxylic acids might contribute to the NMR peaks at high ppm. The peaks above 7 ppm in Figure 5-12 (b) are fluctuating in intensity, which is attributed to further side reactions which occur in the system.
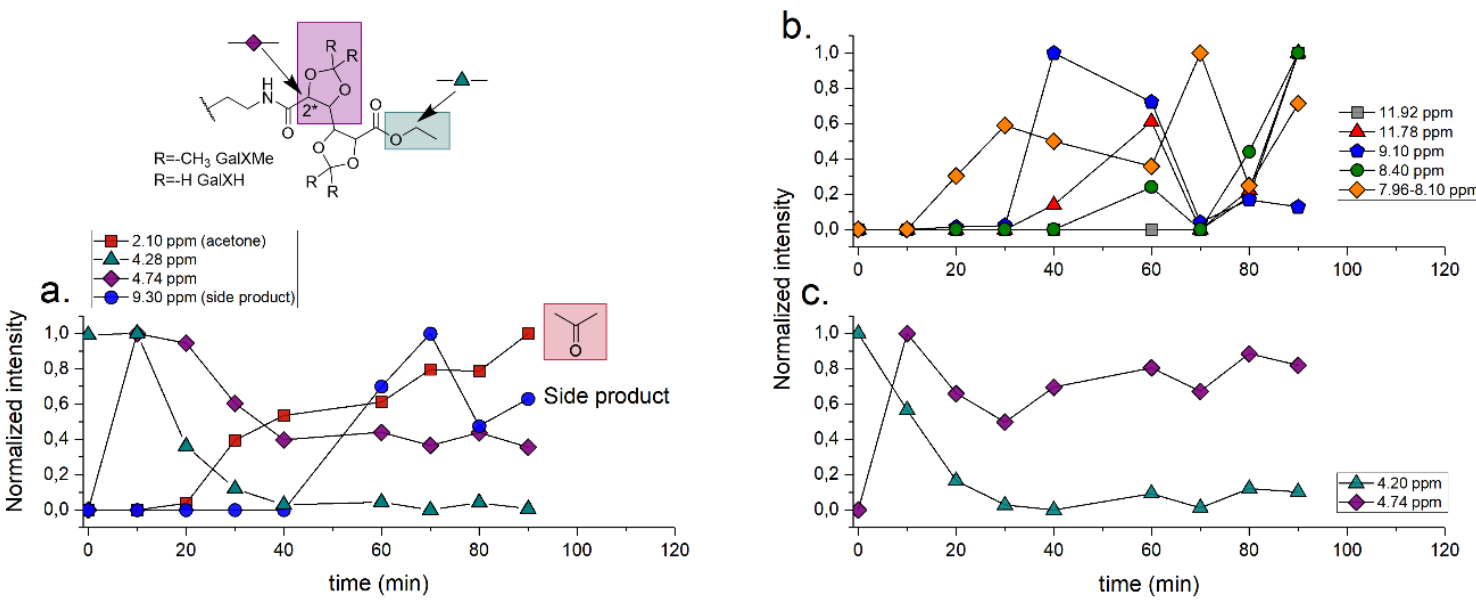

Figure 5-12 Integrated area of peaks present in the ${ }^{1} H$ NMR spectra of (a) PA(GalXMe-HMDA) and $(b, c)$ $P A(G a l X H-H M D A)$. The reaction was carried out in closed vials The spectra were normalized with respect to GalX acetal protection protons ( $3 \mathrm{H}$ at $1.53 \mathrm{ppm}$ for GalXMe and $2 \mathrm{H}$ at $5.13 \mathrm{ppm}$ for GalXH). The intensities of the collected dataset were further normalized from 0-1.

\subsubsection{Maldi-ToF analysis of polymers.}

Additional structural analysis on the polymer samples was done by Maldi-ToF of PA(HMDA,GalXMe) (Figure 5-13 (a)) and PA(HMDA,GalXH) (Figure 5-13 (b)). The presence of repeating unit 370.21 Da for GalXMe polyamide and 314.15 Da for GalXH polyamide confirms the structure of the synthesized polyamides. The graphical representation of the structure of every distribution is specified in Figure 2-6 in Chapter 2.

a.

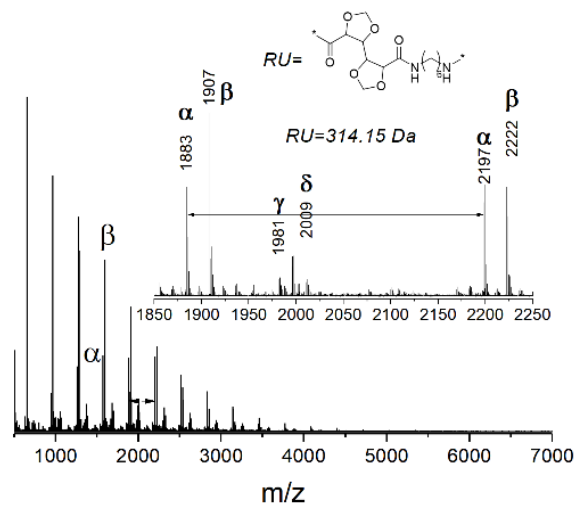

b.

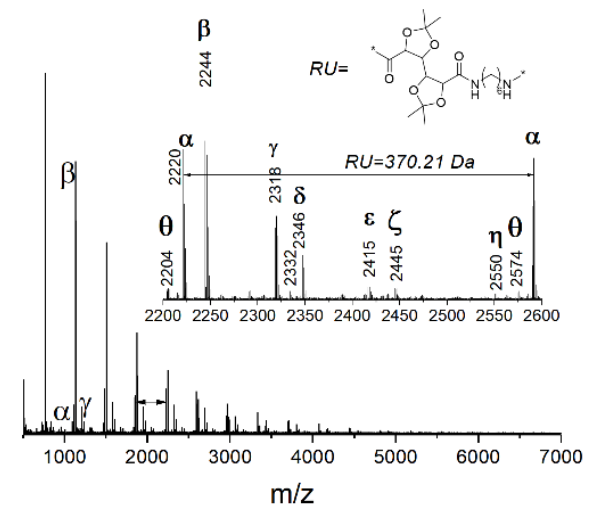

Figure 5-13 Maldi-Tof recorded in reflectron mode with dithranol as a matrix and NaTFA as a salt (a) PA(HMDA,GalXH) (GPC in HFIP: $M_{n}=24000 \mathrm{~g} \cdot \mathrm{mol}^{-1}, \quad D=4.32, \quad R U=\mathrm{C}_{14} \mathrm{H}_{22} \mathrm{O}_{6} \mathrm{~N}_{2}$ ) and (b) $P A\left(H M D A\right.$, GalXMe) (GPC in HFIP: $M_{n}=23000 \mathrm{~g} \cdot \mathrm{mol}^{-1}, D=2.50, R U=\mathrm{C}_{18} \mathrm{H}_{30} \mathrm{O}_{6} \mathrm{~N}_{2}$ ). 


\section{Chapter 5}

As reported earlier, the main distributions can be assigned to linear polyamide chains terminated with ester groups of GalX $(\alpha)$ and cyclic chains $(\beta)$. Distributions $\gamma-\zeta$ correspond to linear polyamide chains which are terminated by ethylated and diethylated amines.

Interestingly, the distributions $\eta$ and $\theta$ in Figure 5-13 correspond to chains which contain one GalXMe deprotected acetal (side product (2) in Figure 5-1). The $\eta$ distribution can be assigned to cyclic chains and $\theta$ to linear chains (Figure 5-14). The presence of those two distributions containing partially deprotected GalXMe product additionally confirms that during the polymerization free hydroxyl groups are formed which may lead to the earlier observed crosslinking upon reaction with diamines. ${ }^{13}$ By consequence, those side products are built into the polymer backbone and therefore cannot be removed by the means of extraction. The fact that those side products are visible in the polymer sample means that their reactivity is limited. If they were highly reactive species, they would react shortly after the formation and therefore would not be detectable.

$\eta$

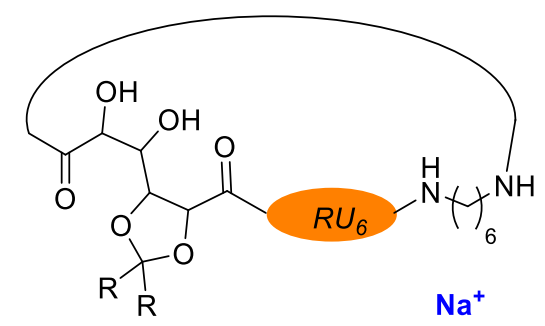

$\theta$

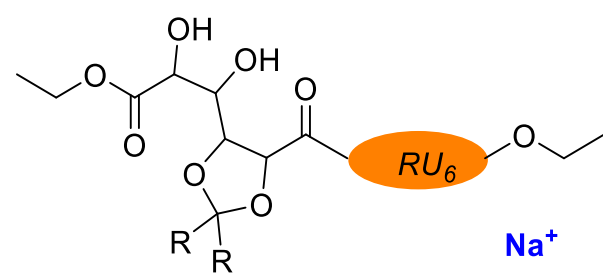

Cyclic chains with partially deprotected GalX

Figure 5-14 Two distributions representing the confirmed presence of the partially deprotected chains in the polymer samples on Maldi-ToF spectrum. $\mathrm{R}$ - $\mathrm{H}$ for GalXH, - $\mathrm{CH}_{3}$ for GalXMe.

The Maldi-ToF analysis of PA(HMDA,GalXH) Figure 5-13(b) revealed the presence of multiple distributions, however, only four of them could be identified: cyclic distribution $(\beta)$ and ethyl ester terminated linear polyamide $(\alpha)$ as well as two distributions with ethylated or diethylated amine end groups $(\gamma, \zeta)$. This, together with previous NMR and TGA-MS analysis results, is an indication that GalXH thermally degrades according to a different mechanism than GalXMe. The tendency to give a crosslinked product hampers further identification of side products by Maldi-ToF technique, since the technique is only effective for soluble polymeric chains. GalXMe polymers release acetone during the partial deprotection and results in detectable free $\mathrm{OH}$ groups. However, for $\mathrm{GalXH}$ polymers free $\mathrm{OH}$ groups were 
not detected by Maldi-ToF. This observation is in line with the LC-MS results on diamide model compounds where the partially deprotected acetal (distribution $\gamma$ in Figure 5-13(b)) has a very low intensity in comparison to other side products formed during the diamide preparation. This could be attributed to the fact that different fragments are eliminated from the GalXH molecule, additional reactions on $\alpha$ and $\beta$ carbons occur while the acetal is still attached to the backbone (e.g. substitution) or the free hydroxyl groups are so reactive that they readily react further with other molecules present in the reaction environment. The latter statement is supported by the fact that in comparison to the isopropylidene derivative GalXMe, the methylene acetal of GalXH is less bulky thus does not hinder neighboring hydroxyl pendant groups, which by consequence make them more accessible.

\subsubsection{GPC analysis of polymers.}

The GPC chromatograms in Figure 5-15 represent a typical molecular weight distribution of PA(HMDA,GalXH) and PA(HMDA,GalXMe) prepared via ester-amide bond exchange reactions. The shape of the PA(HMDA,GalXH) chromatogram shows a characteristic shoulder at lower elution times, which corresponds to higher molecular weight fractions. As hypothesized earlier, ${ }^{13}$ this shoulder is a result of the high reactivity of the ethyl ester group in GalXH and the presence of side reactions localized at the acetal of the GalXH backbone, which results in crosslinking/branching.

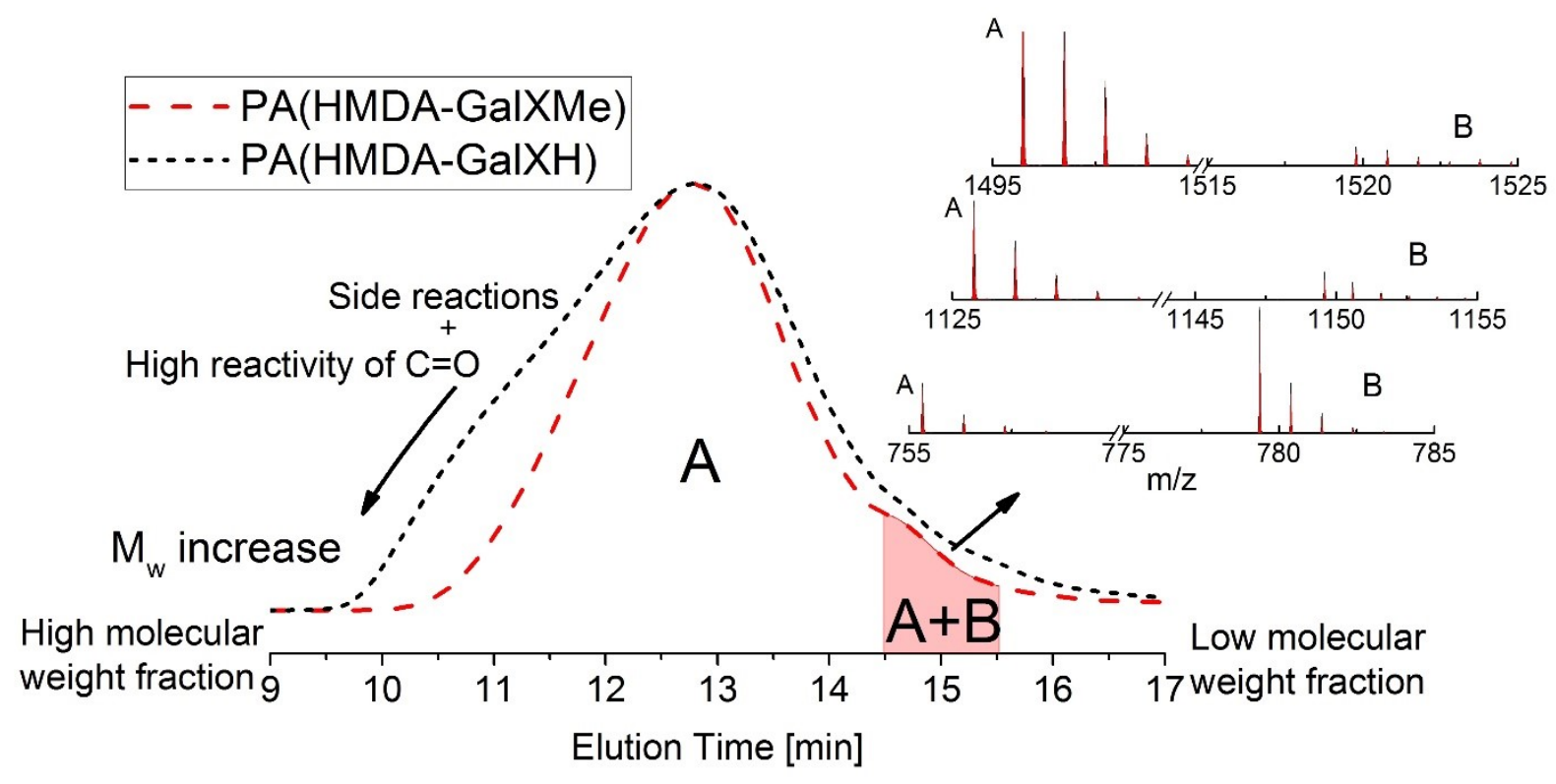

Figure 5-15 GPC traces (HFIP, RI detection, PMMA standards) of PA(HMDA, GalXH) $\left(M_{n}=24000 \mathrm{~g} \cdot \mathrm{mol}^{-1}\right.$, $\left.M_{w}=103700 \mathrm{~g} \cdot \mathrm{mol}^{-1}, Ð=4.32\right)$ and $P A(H M D A, G a l X M e)\left(M_{n}=23000 \mathrm{~g} \cdot \mathrm{mol}^{-1}, M_{w}=57500 \mathrm{~g} \cdot \mathrm{mol}^{-1}, D=2.5\right)$ and Maldi-ToF of low molecular weight PA(HMDA,GalXMe) (GPC in HFIP: $\left.M_{n}=1800 \mathrm{~g} \cdot \mathrm{mol}^{-1}, D=4.1\right)$ for different $\mathrm{m} / \mathrm{z}$ ranges (755-785 Da, 1125-1155 Da and 1495-1525 Da) demonstrating how the ratio of the two distributions changes with regard to molecular weight. A - linear distribution, B-cyclic distribution. 


\section{Chapter 5}

On the GPC chromatogram recorded for PA(HMDA,GalXMe) the appearance of a shoulder at higher elution times was perceived, i.e. in the oligomer region of the polymer (see Figure 5-15). Maldi-Tof analysis in Figure 5-15(a) reveals that distribution B attributed to cyclic polymer has a higher intensity at low molecular weights while distribution A attributed to linear polymer becomes predominant at higher molecular weights (the right part of Figure 515 shows the evolution of both series as a function of molecular weight). The combination of GPC and Maldi-Tof results leads to the conclusion that the low molecular weight shoulder in GPC can be attributed to the presence of cyclic polymeric chains. While the low molecular fraction of PA(HMDA,GalXMe) is rich in cyclic polymeric chains, in PA(HMDA,GalXH) they are distinguishable but do not constitute a predominant distribution.

Based on the results of both the model reactions and the polymerizations, we observe that GalXH is thermally more labile and thus more challenging to incorporate in polyamides via ester-amide exchange melt polycondensation than GalXMe. This thermal acetal stability of esters is opposite to earlier results about the stability of carbohydrate-derived acetals under acidic hydrolysis conditions, where GalXH acetals stayed intact 8, 9, 11, 25, 26 while GalXMe acetals degraded. ${ }^{10,12,27-30}$ This opposite behavior depending on the applied reaction conditions can be explained by the occurrence of different underlying reaction mechanisms: thermal degradation of esters seems to happen via radical processes (Figure 5-16 (a) and (b)) while acidic hydrolysis goes via cationic intermediates (Figure 5-16 (c) and (d)). ${ }^{31}$ A cationic intermediate is more stable when a tertiary carbocation is formed (GalXMe, Figure 5-16 (c)) compared to a primary carbocation (GalXH, Figure 5-16 (d)). Radical degradation of dioxolane moieties typically includes the extraction of a hydrogen. As it is presented in Figure 5-16, GalXH (b) has more hydrogens which could potentially be extracted than GalXMe (a). 
a.

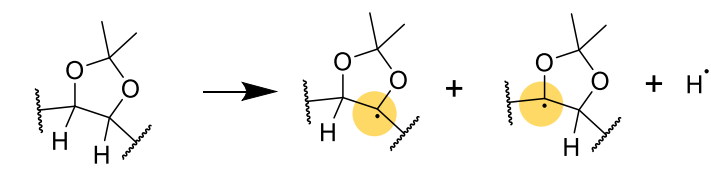

b.

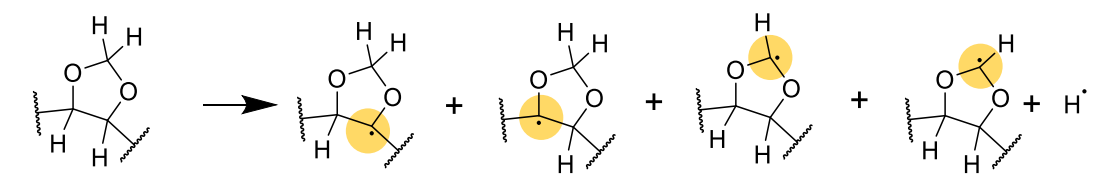

C.

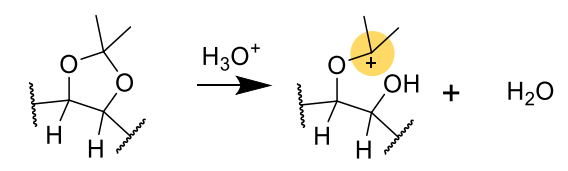

d.
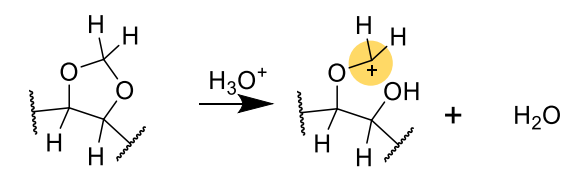

Figure 5-16 Reaction scheme representing two main degradation mechanisms: radical a. for GalXMe moieties, b. for GalXH moteties and cataionic c. for GalXMe moieties, d. for GalXH moteties.

\subsection{Conclusions}

TGA-MS analysis of the polymerization of 2,3:4,5-di-O-isopropylidene-galactaric acid (GalXMe) and 2,3:4,5-di-O-methylene-galactaric acid (GalXH) salts with 1,6hexamethylene diamine revealed that both salts degrade upon melting. The identified molecular fragments confirmed that the degradation of the acetal moieties as well as the decarboxylation of dicarboxylic acid groups are the main reactions contributing to the decomposition. Alternatively, GalX polyamides could be successfully prepared by another method, namely condensation of diethyl esters and diamines. However, some thermal degradation processes and side reactions occur upon polymerization and detailed studies were executed both on model compounds (GPC, NMR and LC-MS) and on polymers (NMR, Maldi-ToF and GPC) to find their origin.

In line with the TGA-MS results, it can be concluded that a small fraction of degraded acetal structure formed upon heating of GalX leads to the occurrence of additional reactive sites on the backbone (e.g. hydroxyl groups, double bonds) and therefore the GalX monomers are not purely difunctional anymore, explaining branching (broadening of $Đ$ ) and crosslinking (gelation) phenomena. It is hypothesized that during melt condensation the GalXH acetal is more prone to partial deprotection of the acetal groups than GalXMe, which is opposite to the effect of acidic hydrolysis. The branching / crosslinking phenomenon is further supported by the appearance of a high molecular weight shoulders in the GPC spectra of GalXH polymers, which is absent for GalXMe polymers. NMR and Maldi-ToF analysis confirmed 


\section{Chapter 5}

the above observations. Besides the decomposition of the GalX protecting groups, Maldi-ToF revealed the occurrence of other side reactions like cyclization and ethylation / diethylation of amines.

Notwithstanding degradation and side reactions are happening during melt polymerization of GalX diethylesters with diamines, they only happen to a limited extend since polyamides with molecular weights above $20000 \mathrm{~g} \cdot \mathrm{mol}^{-1}$ could be prepared successfully.

\subsection{Materials and methods}

\subsubsection{Materials}

Diethyl 2,3:4,5-di- $O$-isopropylidene-galactarate $>99 \%$, diethyl 2,3:4,5-di- $O$-methylenegalactarate $>99 \%, 2,3: 4,5$-di- $O$-isopropylidene-galactaric acid $>99 \%$ and 2,3:4,5-di- $O$ methylene-galactaric acid $>99 \%$ were delivered by Royal Cosun. 1,6-hexamethylenediamine (HMDA) 98\%, benzyl amine (BnA) 99\%, Irganox 1330, acetonitrile-d $\mathrm{d}_{3}\left(\mathrm{ACN}-\mathrm{d}_{3}\right)$, dithranol, NaTFA and KTFA were purchased from Sigma Aldrich and used as received. Silica gel (60$200 \mu \mathrm{m}, 60 \AA$ ) , 1,1,1,3,3,3-hexafluoro-2-propanol (HFIP), dimethyl sulfoxide (DMSO) 99.7\%, DMSO- $\mathrm{d}_{6}$ and $\mathrm{CDCl}_{3}$ were purchased from Acros Organics. Tetrahydrofuran (THF), n-hexane, ethyl acetate, acetonitrile $(\mathrm{ACN})$, acetone, chloroform $\left(\mathrm{CHCl}_{3}\right)$ and ethanol were purchased from Biosolve.

\subsubsection{Methods}

1,6-hexamethylenediamine-2,3:4,5-di- $O$-isopropylidene-galactaric acid salt, 1,6hexamethylenediamine-2,3:4,5-di- $O$-methylene-galactaric acid salt, poly(hexamethylene2,3:4,5-di- $O$-isopropylidene-galactaramide) and synthesis of poly(hexamethylene-2,3:4,5-di$O$-methylene-galactaramide) were synthesized according to procedures presented in Chapter 2 and Chapter 4.

Dibenzyl 2,3:4,5-di-O-isopropylidene-galactaramide. To a $100 \mathrm{~mL}$ three-necked round bottom flask equipped with a vacuum tight mechanical stirrer, a vigreux column and a distillation condenser were added diethyl 2,3:4,5-di-O-isopropylidene-galactarate $(5.00 \mathrm{~g}, 14$ mmol), benzylamine $(3.00 \mathrm{~g}, 28 \mathrm{mmol})$ and Irganox $1330(0.07 \mathrm{~g}, 0.1 \mathrm{wt} \%)$ as an antioxidant. The reactants were slowly heated till $220{ }^{\circ} \mathrm{C}$ and kept at this temperature for 8 hours. The mixture was cooled down and purified by liquid chromatography with silica gel using hexane:ethyl acetate in a 3:7 ratio as eluent. The solid, white product was filtered and dried under vacuum at $80{ }^{\circ} \mathrm{C}$ for $24 \mathrm{~h}$, affording $4.098 \mathrm{~g}(8.8 \mathrm{mmol}, 60.6 \%)$. 
Melting point: $201-202{ }^{\circ} \mathrm{C}$

${ }^{l} \mathrm{H} \mathrm{NMR}\left(300 \mathrm{MHz}, \mathrm{DMSO}-d_{6}\right) \delta(\mathrm{ppm}): 7.19\left(10 \mathrm{H}, \mathrm{m},-\mathrm{NH}-\mathrm{CH}_{2}-\mathrm{C}_{6} \mathrm{H}_{5}\right), 6.88(2 \mathrm{H}, \mathrm{s},-\mathrm{NH}-)$, $4.76(2 \mathrm{H}, \mathrm{m}-\mathrm{CH}-\mathrm{O}-), 4.52(2 \mathrm{H}, \mathrm{m}-\mathrm{CH}-\mathrm{O}-), 4.42\left(4 \mathrm{H}, \mathrm{d}, J=6 \mathrm{~Hz}-\mathrm{NH}-\mathrm{CH}_{2}-\mathrm{C}_{6} \mathrm{H}_{5}\right), 1.49(6 \mathrm{H}$, $\left.\mathrm{s}, \mathrm{C}-\mathrm{CH}_{3}\right), 1.42\left(6 \mathrm{H}, \mathrm{s}, \mathrm{C}-\mathrm{CH}_{3}\right)$.

${ }^{13} \mathrm{C} \mathrm{NMR}(75 \mathrm{MHz}, \mathrm{DMSO}) \delta(\mathrm{ppm}): 171.0(2 \mathrm{C}, \mathrm{C}=\mathrm{O}), 139.7\left(2 \mathrm{C},-\mathrm{NH}-\mathrm{CH}_{2}-\mathrm{C}_{6} \mathrm{H}_{5}\right), 128.7$ (4C,- $\left.\mathrm{NH}-\mathrm{CH}_{2}-\mathrm{C}_{6} \mathrm{H}_{5}\right), 127.7$ (4C, - $\left.\mathrm{NH}-\mathrm{CH}_{2}-\mathrm{C}_{6} \mathrm{H}_{5}\right), 127.2\left(2 \mathrm{C},-\mathrm{NH}-\mathrm{CH}_{2}-\mathrm{C}_{6} \mathrm{H}_{5}\right), 111.0(2 \mathrm{C}$, $C-\mathrm{CH}_{3}$ acetal), 78.9 (2C, -CH-O-), 76.5 (2C, $\left.-\mathrm{CH}-\mathrm{O}-\right), 42.4\left(2 \mathrm{C},-\mathrm{NH}-\mathrm{CH}_{2}-\mathrm{C}_{6} \mathrm{H}_{5}\right), 27.1$ (2C, $\left.-\mathrm{C}-\mathrm{CH}_{3}\right), 26.0\left(2 \mathrm{C},-\mathrm{C}-\mathrm{CH}_{3}\right)$.

$L C-M S$ (ESI) $m / z$ : [M+1] calcd. for $\mathrm{C}_{22} \mathrm{H}_{24} \mathrm{O}_{6} \mathrm{~N}_{2}, 469.23 \mathrm{~g} / \mathrm{mol}$; found: $469 \mathrm{~g} / \mathrm{mol}$.

Dibenzyl 2,3:4,5-di-O-methylene-galactaramide. To a 100mL three-necked round bottom flask equipped with a vacuum tight mechanical stirrer, a vigreux column and a distillation condenser were added diethyl 2,3:4,5-di-O-methylene-galactarate (4.06 g, $14 \mathrm{mmol}$ ), benzylamine $(3.00 \mathrm{~g}, 28 \mathrm{mmol})$ and Irganox $1330(0.07 \mathrm{~g}, 0.1 \mathrm{wt} \%)$ as an antioxidant. The reactants were slowly heated till $220^{\circ} \mathrm{C}$ and kept at this temperature for 8 hours. The mixture was cooled down and the impurities were dissolved in hexane:ethyl acetate in a 3:7 ratio. The undissolved, solid, white product was filtered and dried under vacuum at $80{ }^{\circ} \mathrm{C}$ for 24 hours, affording $2.96 \mathrm{~g}(7.2 \mathrm{mmol}, 51.3 \%)$.

Melting point: $214-215^{\circ} \mathrm{C}$

${ }^{l} \mathrm{H} \mathrm{NMR}\left(300 \mathrm{MHz}, \mathrm{DMSO}-d_{6}\right) \delta(\mathrm{ppm}): 8.79(2 \mathrm{H}, \mathrm{s},-\mathrm{NH}-), 7.29\left(10 \mathrm{H}, \mathrm{m},-\mathrm{NH}-\mathrm{CH}_{2}-\mathrm{C}_{6} \mathrm{H}_{5}\right)$, $5.18\left(2 \mathrm{H}, \mathrm{s}, \mathrm{O}-\mathrm{CH}_{2}-\mathrm{O}\right), 4.96\left(2 \mathrm{H}, \mathrm{s}, \mathrm{O}-\mathrm{CH}_{2}-\mathrm{O}\right), 4.49$ (2H, m -CH-O-), 4.32 (2H, m-CH-O-), $4.29\left(4 \mathrm{H}, \mathrm{d}, \mathrm{J}=6 \mathrm{~Hz}-\mathrm{NH}-\mathrm{CH}_{2}-\mathrm{C}_{6} \mathrm{H}_{5}\right)$

${ }^{13} \mathrm{C} N M R\left(75 \mathrm{MHz}, \mathrm{DMSO}-d_{6}\right) \delta(\mathrm{ppm}): 170.4(2 \mathrm{C}, \mathrm{C}=\mathrm{O}), 139.7\left(2 \mathrm{C},-\mathrm{NH}-\mathrm{CH}_{2}-\mathrm{C}_{6} \mathrm{H}_{5}\right), 128.7$ (4C, $\left.-\mathrm{NH}-\mathrm{CH}_{2}-\mathrm{C}_{6} \mathrm{H}_{5}\right), 127.9$, (4C, - $\left.\mathrm{NH}-\mathrm{CH}_{2}-\mathrm{C}_{6} \mathrm{H}_{5}\right), 127.3\left(2 \mathrm{C},-\mathrm{NH}-\mathrm{CH}_{2}-\mathrm{C}_{6} \mathrm{H}_{5}\right), 96.1$ (2C, O-C $\left.\mathrm{H}_{2}-\mathrm{O}\right), 79.8$ (2C, -CH-O-), 75.4 (2C, -CH-O-), $42.3\left(2 \mathrm{C},-\mathrm{NH}-\mathrm{CH}_{2}-\mathrm{C}_{6} \mathrm{H}_{5}\right)$

$L C-M S$ (ESI) m/z: [M+1] calcd. for $\mathrm{C}_{22} \mathrm{H}_{24} \mathrm{O}_{6} \mathrm{~N}_{2}, 413.17 \mathrm{~g} / \mathrm{mol}$; found: $413 \mathrm{~g} / \mathrm{mol}$.

Synthesis of poly(hexamethylene-2,3:4,5-di-O-isopropylidene-galactaramide) and synthesis of poly(hexamethylene-2,3:4,5-di-O-methylene-galactaramide) in closed vials. An equimolar amount of diethyl 2,3:4,5-di-O-isopropylidene-galactarate or diethyl 2,3:4,5-di-Omethylene-galactarate and 1,6-hexamethylenediamine were placed in $2 \mathrm{~mL}$ crimp-top vials and sealed with an aluminum cap with natural red rubber septa. Vials were flushed with dry nitrogen for 15 minutes, placed on a heating plate at $100{ }^{\circ} \mathrm{C}$ and heated to $220{ }^{\circ} \mathrm{C}$ over the course of 10 minutes. The vials were collected with intervals of 10 minutes and each vial was cooled in liquid nitrogen to stop the reaction. 


\section{Chapter 5}

\subsubsection{Characterization}

The thermal stability, melting temperatures, molecular weight of polyamides, matrix-assisted laser desorption/ionization time-of-flight (Maldi-ToF) were determined/recorded as described in the experimental section in Chapter 2 and liquid chromatography - mass spectrometry in Chapter 3.

TGA-MS measurement was done on a HPR-20 QIC Gas Analysis System from Hiden Analytical connected through an MS inlet to the Mettler Toledo TGA/SDTA 851e. Around $10 \mathrm{mg}$ sample was placed in the measurement pan followed by heating with a heating rate of $10{ }^{\circ} \mathrm{C} / \mathrm{min}$ to the target temperature above the melting point of the sample. The sample is then kept at this temperature for an additional hour.

${ }^{1} H$-NMR $,{ }^{13} C-N M R,{ }^{13} C-N M R / D E P T-135$ and the corresponding 2D spectra were recorded in $\mathrm{CDCl}_{3}, \mathrm{ACN}-\mathrm{d}_{3}, \mathrm{DMSO}-\mathrm{d}_{6}$ on a Bruker $300 \mathrm{MHz}$ spectrometer. ${ }^{1} H \mathrm{H}$ R was recorder with 16 scans and $10 \mathrm{sec}$ pre-scan delay, ${ }^{13} C-N M R$ was recorded using $1 \mathrm{D}$ sequence with powergated decoupling with 8192 scans and $6.50 \mathrm{sec}$ pre-scan delay, ${ }^{13} \mathrm{C}-N M R / D E P T-135$ with polarization transfer, decoupling during acquisition, using shaped pulse for the $180^{\circ}$ pulse on the $\mathrm{fl}$ - channel and $135^{\circ}$ read pulse to give $\mathrm{CH}, \mathrm{CH}_{3}$ positive and $\mathrm{CH}_{2}$ negative signals was recorded with 8192 scans and $6.50 \mathrm{sec}$ pre-scan delay. The corresponding $2 D-H S Q C$ spectra were recorded using the standard Bruker pulse program hsqcetgpsisp2.3 with the following parameters: acquisition TD 2048 (f2) x256 (f1), SW = 9.1 ppm (F2), 166 ppm (F1); $\mathrm{O} 1,1415.59 \mathrm{~Hz} ; \mathrm{O} 2,5657.72 \mathrm{~Hz}$; D1 = $4.84 \mathrm{~s}$; CNST2 = 145; acquisition time, F2 channel, $374.784 \mathrm{~ms}$; F1 channel, $10.238 \mathrm{~ms}$; processing, SI = 1024 (F2, F1), WDW =QSINE, LB = $1.00 \mathrm{~Hz}(\mathrm{~F} 2), 0.30 \mathrm{~Hz}(\mathrm{~F} 1)$; PH_mod = pk; baseline correction $\mathrm{ABSG}=5(\mathrm{~F} 2, \mathrm{~F} 1), \mathrm{BCFW}=$ $1.00 \mathrm{ppm}$, BC_mod $=$ qpol $(\mathrm{F} 2)$, no $(\mathrm{F} 1)$; linear prediction $=$ no $(\mathrm{F} 2)$, LPfr $(\mathrm{F} 1)$. The spectrum was recorded with 32 scans and $6.50 \mathrm{sec}$ pre-scan delay.

$A T R-I R$ analysis was done with a PerkinElmer FTIR/NIR spectrometer Frontier with resolution $4 \mathrm{~cm}^{-1}$ and 16 scans per spectrum. The background scan was recorded with 64 accumulations and resolution $4 \mathrm{~cm}^{-1}$. 


\section{REFERENCES}

1. C. Lavilla, E. Gubbels, A. Martínez de Ilarduya, B. A. J. Noordover, C. E. Koning and S. Muñoz-Guerra, Macromolecules, 2013, 46, 4335-4345.

2. S. Selifonov, A. E. Goetz, M. Scholten and N. Zhou, WO 2010/075330 A1, 2010.

3. M. E. Rogers and T. E. Long, Synthetic methods in step-growth polymers., A John Wiley \& Sons, inc., Hoboken, New Jersey, 2003.

4. J. E. Flannigan and G. A. Mortimer, J. Polym. Sci., Part A: Polym. Chem., 1978, 16, 12211228.

5. E. Hellmann, J. Malluche and G. P. Hellmann, Polym. Eng. Sci., 2007, 47, 1600-1609.

6. J. Malluche, G. P. Hellmann, M. Hewel and H.-J. Liedloff, Polym. Eng. Sci., 2007, 47, 15891599.

7. E. Hellmann, J. Malluche and G. P. Hellmann, Polym. Eng. Sci., 2007, 47, 1600-1609.

8. C. Lavilla, A. Alla, A. Martínez de Ilarduya, E. Benito, M. G. García-Martín, J. A. Galbis and S. Muñoz-Guerra, J. Polym. Sci., Part A: Polym. Chem., 2012, 50, 3393-3406.

9. C. Lavilla and S. Muñoz-Guerra, Polym. Degrad. Stab., 2012, 97, 1762-1771.

10. C. Lavilla and S. Muñoz-Guerra, Green Chem., 2013, 15, 144-151.

11. S. Muñoz-Guerra, C. Lavilla, C. Japu and A. Martínez de Ilarduya, Green Chem., 2014, 16, 1716-1739.

12. T. W. Greene and P. G. M. Wuts, Greene's protective groups in organic synthesis., John Wiley \& Sons, Inc., Hoboken, New Jersey, 2007.

13. A. A. Wróblewska, K. V. Bernaerts and S. M. A. De Wildeman, Polymer, 2017, 124, 252262.

14. N. Ogata, K. Sanui, K. Hosoda and H. Nakamura, J. Polym. Sci., Polym. Chem. Ed., 1977, 15, 1523-1526.

15. J. M. Brown, M. Manley-Harris, R. J. Field and D. E. Kiely, J. Carbohydr. Chem., 2007, 26, 455-467.

16. M. K. Dowd, D. E. Kiely and J. Zhang, Carbohydr Res, 2011, 346, 1140-1148.

17. D. E. Kiely, A. Vishwanathan, B. P. Jarman and M. Manley-Harris, J. Carbohydr. Chem., 2009, 28, 348-368.

18. C. Regano, A. M. De Ilarduya, J. I. Iribarren and S. Munoz-Guerra, J. Polym. Sci., Part A: Polym. Chem., 2000, 38, 2687-2696.

19. P. Ruiz-Donaire, J. J. Bou, S. Munoz-Guerra and A. Rodriquez-Galan, J. Appl. Polym. Sci., 1995, 58, 41-54.

20. J. I. Iribarren, C. Aleman, J. J. Bou and S. Munoz-Guerra, Macromolecules 1996, 29, $4397-$ 4405.

21. J. J. Bou, A. Rodriquez-Galan and S. Munoz-Guerra, Macromolecules, 1993, 26, 5664-5670.

22. B. P. Jarman, D. E. Kiely, M. Manley-Harris and B. K. Nicholson, J. Carbohydr. Chem., 2009, 28, 107-123.

23. C. G. Sauer, I. Barnes, K. H. Becker, H. Geiger, T. J. Wallington, L. K. Christensen, J. Platz and O. J. Nielsen, J. Phys. Chem. A, 1999, 103, 5959-5966.

24. W. B. Guenther and W. D. Walters, J. Am. Chem. Soc., 1951, 73, 2127-2131.

25. C. Lavilla, A. Alla, A. M. de Ilarduya, E. Benito, M. G. Garcia-Martin, J. A. Galbis and S. Munoz-Guerra, Biomacromolecules, 2011, 12, 2642-2652.

26. C. Lavilla, A. Alla, A. Martínez de Ilarduya and S. Muñoz-Guerra, Biomacromolecules, 2013, 14, 781-793.

27. C. Rosu, I. I. Negulescu, R. Cueto, R. Laine and W. H. Daly, J. Macromol. Sci., Part A Pure Appl. Chem., 2013, 50, 940-952.

28. R. V. Gómez and O. Varela, Macromolecules, 2009, 42, 8112-8117.

29. R. Marín and S. Muñoz-Guerra, J. Polym. Sci., Part A: Polym. Chem., 2008, 46, 7996-8012.

30. S. Dhamaniya and J. Jacob, Polymer, 2010, 51, 5392-5399.

31. J. March, Advanced organic chemistry: reactions, mechanism, and structure, John Wiley \& Sons, New York, 4th edn., 1992. 



\section{Chapter 6 DIOXOLANE MOIETIES AND THEIR INTERACTIONS}

Abstract

This chapter focuses on the interactions between the dioxolane motifs and other functional groups present in polyamides containing GalX. The electronegative atoms present in the cyclic structures have a major influence on the efficiency of hydrogen bonding. The consequences and repercussions of the incorporation of cyclic moieties in aliphatic polyamides are studied by FT-IR and will be briefly related to the crystallinity of the copolyamides. Two different molecules are investigated namely i-propylidene- and methylene- acetals of diethyl galactarate (GalXMe and GalXH respectively) and the corresponding (poly)amides. The amide protons of such polyamides tend to form hydrogen bonding with the acetal oxygen of neighboring GalX (intramolecular) and therefore prevents interchain hydrogen bonding. In consequence, the efficiency of hydrogen bonding decreases. Another significant factor is the bulky structure of GalX, which suppreses the crystallinity of polyamides to an extent which depends on the structure of the acetal itself. The GalXMe suppresses the crystallinity to a higher extent than GalXH 



\subsection{Introduction}

GalX possesses two dioxolane rings or substituted dioxolane rings connected by a carboncarbon linkage, which contribute to very specific properties of the molecule and polymers therefrom. The GalX molecule has 4 chiral carbons; however it has a configuration meso which makes it optically not active due to its exact mirror image. Due to the rigidity of the dioxolane rings GalX can contribute to improved thermal properties like increased glass transition temperature and increased melting temperature. ${ }^{1-3}$ The bulky character of ring structures incorporated into linear polyesters or polyamides can highly hamper close chain packing which is characteristic for semi-crystalline materials, challenging crystallization and lowering the melting temperature. ${ }^{4}$ Indeed, upon the incorporation of GalX into polyamides (based on $\mathrm{DSC}^{5,6}$ ) or polyesters (WAXD analysis of crystals ${ }^{2,4}$ ) the crystallinity of those polymers is highly suppressed and drops to almost zero already at $30 \%$ of these moieties in the copolymer. The consequence of this GalX contribution has predominant influences on the mechanical properties of polymers therefrom and alters its appearance to transparent.

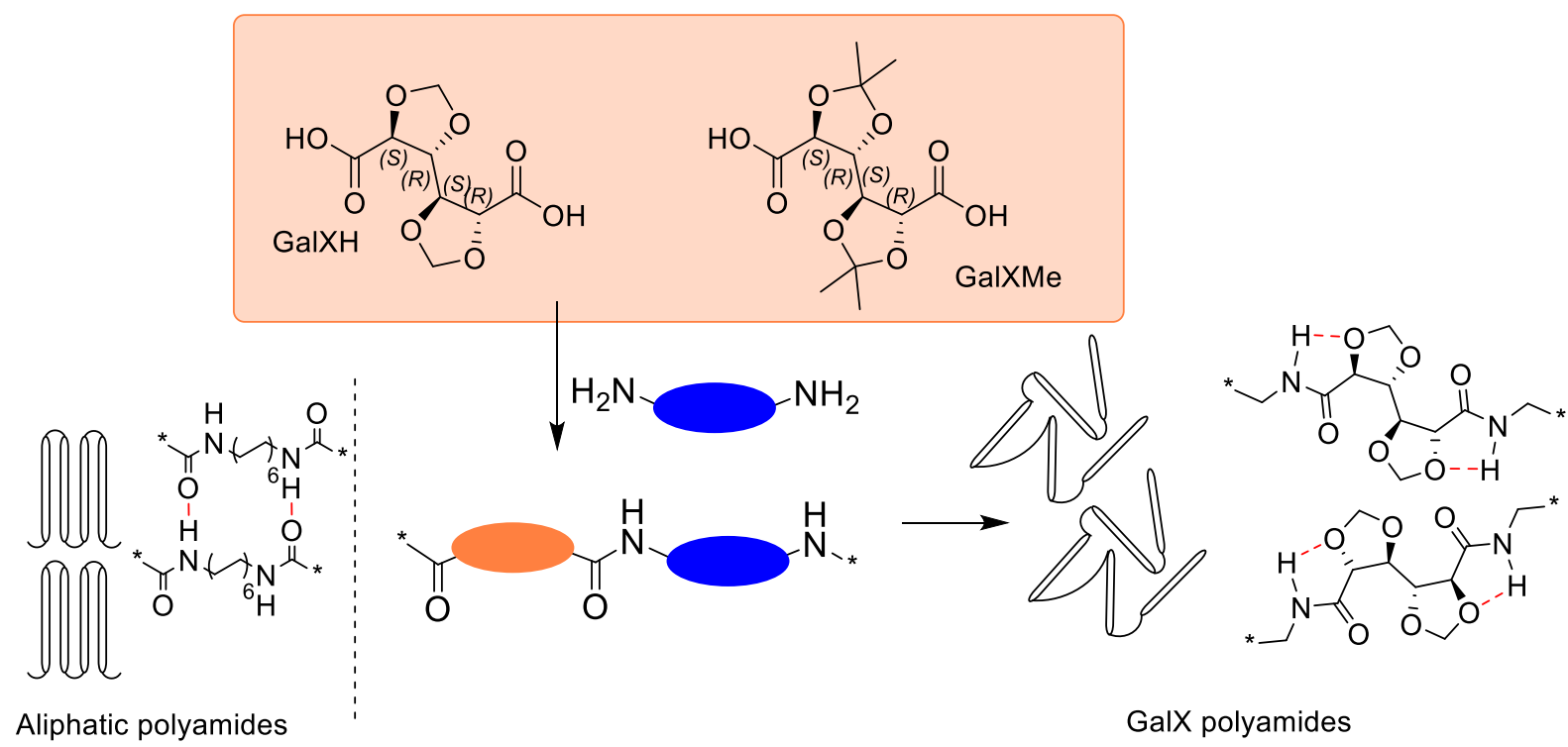

Figure 6-1 General scheme representing a typical hydrogen bonding network present in aliphatic polyamides and the possible hydrogen bonding occurring in GalX-based polyamides.

Available literature reports show that the exact structure of those biacetalized moieties controls a lot of properties starting from the hydrolytic or thermal stability ${ }^{6-11}$ to solubility. ${ }^{12}$ The important factors are on one hand the structure of acetal protection (methylene or ipropylidene acetal) and on the other hand the ring structure and orientation e.g. 6-membered 1,3-dioxane fused rings of biacetalized D-mannaric (ManX) or biacetalized D-glucaric acid 


\section{Chapter 6}

$(\mathrm{GluX})^{13}$ moieties give different results than for GalX, which has two 1,3-dioxolane rings separated by C-C bond.

Despite that the influence of cyclic acetal moieties on the properties of polymers therefrom is already described, the exact interactions that lead to it, are still not fully understood. Especially with regard to polyamides, whose properties are hugely determined by the interchain hydrogen bonding, which can be significantly disrupted by the presence of additional electronegative atoms in the structure and bulkiness of the cyclic moieties (see Figure 6-1). ${ }^{14-17}$ Moreover, biacetalized carbohydrate-based polyamides are not fully investigated and far most of the studies published through the last couple of decades focused on polyesters and copolyesters or polyurethanes ${ }^{18-20}$ rather than polyamides. In contrast, isohexide-based polyamides are much broader investigated, also with regard to the influence on the crystalline domains as well as the interactions with other moieties in the polymer, including theoretical calculations and conformation analysis. ${ }^{21-24}$

\subsection{Structure and synthesis of polyamides}

In this chapter two different polymeric materials are studied; homopolymers containing GalX and 1,12-dodecanediamine (DDDA), and copolymers containing two acid functionalized compounds, GalX and sebacic acid (SA) in different ratio's combined with 1,12dodecanediamine (DDDA) (see Figure 6-2 and Table 6-1). The first material with DDDA is a polymer, which is fully amorphous, highly transparent and brittle. The second type of material, a series of copolymers, is a semi-crystalline material, in which the crystallinity strongly depends on the amount of the GalX.

\section{Homopolymers}<smiles>[R]C1([R])OC(C(C)=O)C(C2OC([R])([R])OC2C(=O)NNCNC)O1</smiles>

$\operatorname{PA}\left(D_{D D A}{ }_{100}\right.$, GalXR $\left._{100}\right)$

Molar ratio of monomers

Figure 6-2 Polyamides studied in this work.
Copolymers

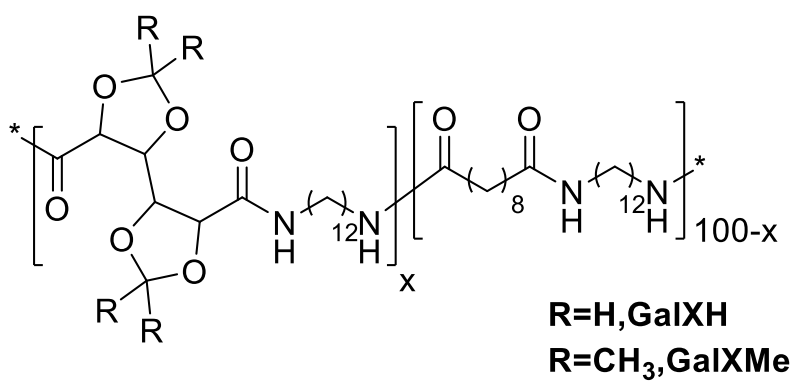


The structures of those polymers were elucidated by liquid NMR and Maldi-ToF and were in detail described in Chapter 2 and Chapter 4 as well as in the synthetic approach.

Table 6-1 Characteristics of homo- and copolyamides from sebacic acid (SA), GalX (GalXH and GalXMe) and 1,12-dodecanediamine (DDDA).

\begin{tabular}{|c|c|c|c|c|c|c|}
\hline Entry & Composition $^{a}$ & $\begin{array}{l}M_{n}{ }^{b} \\
(\mathrm{~kg} / \mathrm{mol})\end{array}$ & $\boldsymbol{\boxplus}^{b}$ & $\begin{array}{l}T_{m}{ }^{c} \\
\left({ }^{\circ} \mathrm{C}\right) \\
\end{array}$ & $\begin{array}{c}\Delta H_{m}{ }^{c} \\
(J / g)\end{array}$ & $\begin{array}{l}X_{W A X D^{e}} \\
(\%)\end{array}$ \\
\hline$P A 1$ & $\mathrm{DDDA}_{93} / \mathrm{SA}_{100}$ & 11.0 & 2.7 & $192 / 200$ & 67 & 67 \\
\hline coPA2 & $\mathrm{GalXH}_{9} / \mathrm{DDDA}_{101} / \mathrm{SA}_{91}$ & 14.0 & 2.4 & $184 / 195$ & 76 & 75 \\
\hline $\operatorname{coPA3}$ & $\mathrm{GalXH}_{27} / \mathrm{DDDA}_{105} / \mathrm{SA}_{73}$ & 21.0 & 2.6 & $180 / 187$ & 54 & 67 \\
\hline coPA4 & $\mathrm{GalXH}_{49} / \mathrm{DDDA}_{105} / \mathrm{SA}_{51}$ & 31.0 & 2.7 & $170 / 180$ & 23 & 44 \\
\hline$P A 5$ & $\mathrm{DDDA}_{102} / \mathrm{GalXH}_{100}$ & 31.0 & 5.7 & 189 & 29 & $\mathrm{~N} / \mathrm{A}^{*}$ \\
\hline coPA6 & $\mathrm{GalXMe}_{8} / \mathrm{DDDA}_{103} / \mathrm{SA}_{92}$ & 14.0 & 4.0 & $185 / 192$ & 60 & 68 \\
\hline $\operatorname{coPA} 7$ & $\mathrm{GalXMe}_{24} / \mathrm{DDDA}_{105} / \mathrm{SA}_{76}$ & 21.0 & 6.0 & 171 & 31 & 49 \\
\hline соPA8 & $\mathrm{GalXMe}_{44} / \mathrm{DDDA}_{105} / \mathrm{SA}_{56}$ & 31.0 & 5.9 & 146 & 11 & 40 \\
\hline PA9 & $\mathrm{DDDA}_{102} / \mathrm{GalXMe}_{100}$ & 21.0 & 2.0 & - & - & - \\
\hline
\end{tabular}

*N/A The crystallinity of this sample is very low and due to a different WAXD pattern it cannot be compared with the crystallinity of $(c o) P A(1-4)^{\text {a }}$ composition of the copolymer as determined by NMR, subscripts represent molar \% of acid functionalized monomers (GalX and SA) and amine functionalized monomer (DDDA) ${ }^{b}$ Determined by GPC in HFIP with PMMA standards ${ }^{\mathrm{C}}$ Determined by DSC of films casted from HFIP solution after $1^{\text {st }}$ run with heating rate $1^{\circ} \mathrm{C} / \mathrm{min}$, ${ }^{\mathrm{e}}$ crystallinity calculated by XRD

\subsection{Molecular interactions of amide protons}

PAs are well-known for their organized crystalline structure which is based on strong hydrogen bonds between $\mathrm{H}(\mathrm{N}-\mathrm{H})$ and $\mathrm{O}(\mathrm{C}=\mathrm{O})$ of adjacent amide moieties. These amide motifs can be intra- and interchain in nature, i.e. amide groups belonging to a single chain or different chains (Figure 6-3).

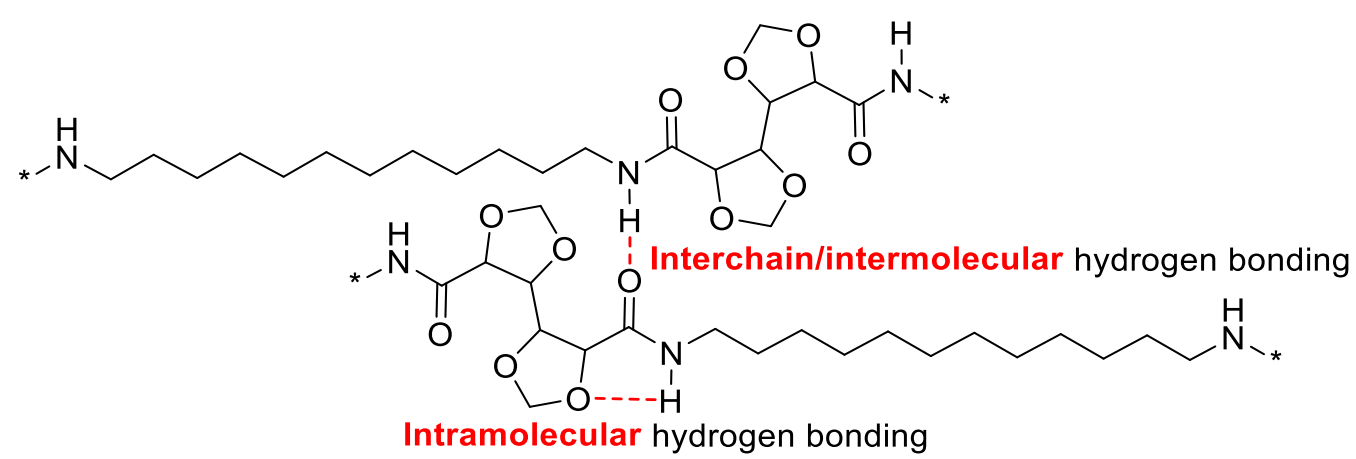

Figure 6-3 The definition of interchain and intramolecular hydrogen bonding which can be formed in the GalXbased polyamides.

Infrared analysis allows to characterize hydrogen bonding, their efficiency as well as the conformation of molecules or their location (in or outside the crystals). Figure 6-4 presents the region of the N-H stretching vibration of two GalX polyamides and an aliphatic highly crystalline polymer containing 1,12-dodecanediamine and sebacic acid PA(DDDA,SA). The latter one shows a peak of the strongly hydrogen bonded N-H vibration (at $3306 \mathrm{~cm}^{-1}$ ). In aliphatic polyamides weakly hydrogen bonded amide protons occur rarely, but are present in 


\section{Chapter 6}

the amorphous phase and prominent at elevated temperature e.g. above the melting point ${ }^{25}$ when they can be easily spotted by FT-IR spectroscopy. However, on the spectra of GalX homopolyamide it is clearly visible that an additional peak above $3400 \mathrm{~cm}^{-1}$ occurs which belongs to weakly hydrogen bonded $\mathrm{N}-\mathrm{H}$. The peaks of the investigated GalX polyamides are much broader than for the aliphatic reference of PA(DDDA,SA), what is typically associated with increased motion due to weakening of hydrogen bonding. ${ }^{26}$ However, the N$\mathrm{H}$ stretching mode may also be influenced by additional interactions between $\mathrm{O}(\mathrm{O}-\mathrm{C}$ $\mathrm{O} /$ acetal) and $\mathrm{H}(\mathrm{N}-\mathrm{H})$ as sketched in Figure 6-4. Due to the close proximity and limited motion of such local intramolecular interactions, the $\mathrm{NH}$-stretch vibration will shift to a lower wavenumber $\left(3281 \mathrm{~cm}^{-1}\right)$ as witnessed by the FTIR spectrum in Figure 6-4. Simultaneously these interactions hamper the ability of GalX polymers to form typical amide-amide hydrogen bonding and therefore hampering crystallization, supporting the presence of a second, broad (i.e. more mobile and less efficiently hydrogen bonded) $\mathrm{NH}$ stretch band at higher wavenumber, $3425 \mathrm{~cm}^{-1}$. It is much more pronounced for GalXMe due to an additional steric effect of the bulkier isopropylidene acetal, hampering amide-amide hydrogen bonding even more.

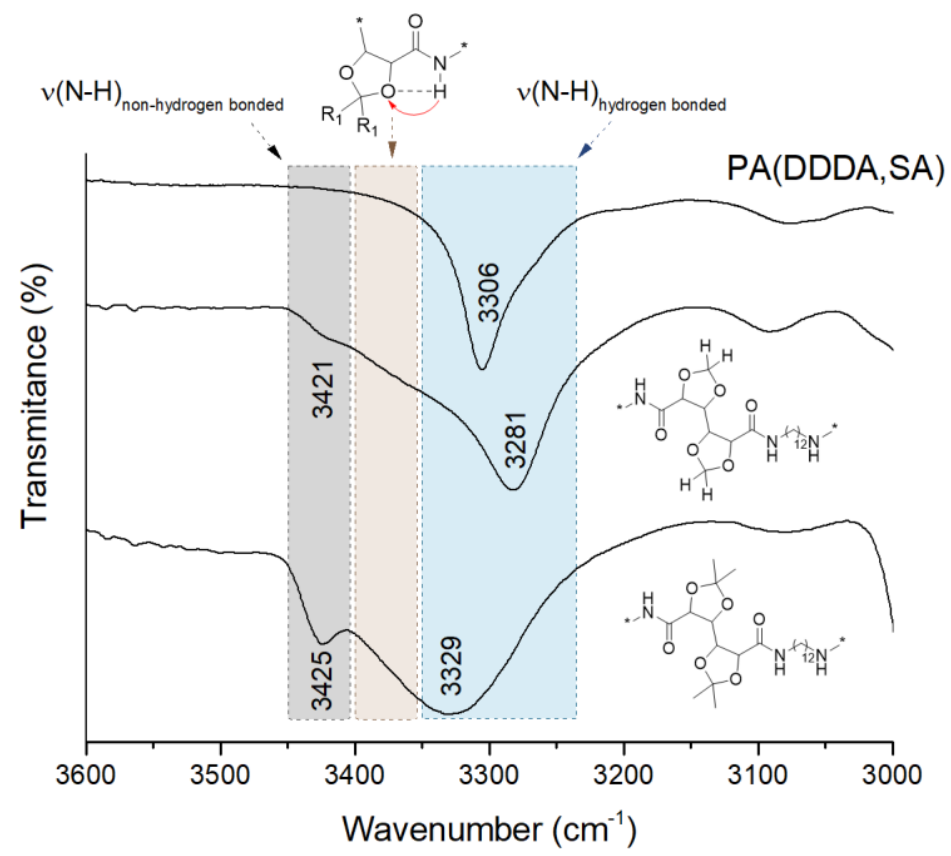

Figure 6-4 FT-IR spectrum of the stretching vibration of $\mathrm{N}-\mathrm{H}\left(3600-3000 \mathrm{~cm}^{-1}\right)$ in homopolyamides PA(DDDA,SA) PA1 top, PA(DDDA,GalXH) PA5 middle and PA(DDDA,GalXMe) PA9 bottom. 


\subsection{Stabilization of GalX amide conformations by the formation of intramolecular hydrogen bonds between $\mathrm{H}(\mathrm{N}-\mathrm{H})$ GalX and acetal}

In order to elucidate the structure of GalX in polyamides the potential energy surfaces (PES) were calculated for both GalX esters and GalX amides. The difference in those surfaces provides information on how the presence of additional amide hydrogens $(\mathrm{N}-\mathrm{H})$ influences the conformation of the molecule. The energy surfaces were obtained by a relaxed scan along two dihedrals with redundant coordinates (Figure 6-5). The first scanned coordinate is the dihedral angle between two acetal rings $\Psi$ and the second scanned coordinate is the dihedral angle between ring and carbonyl $\Phi$.
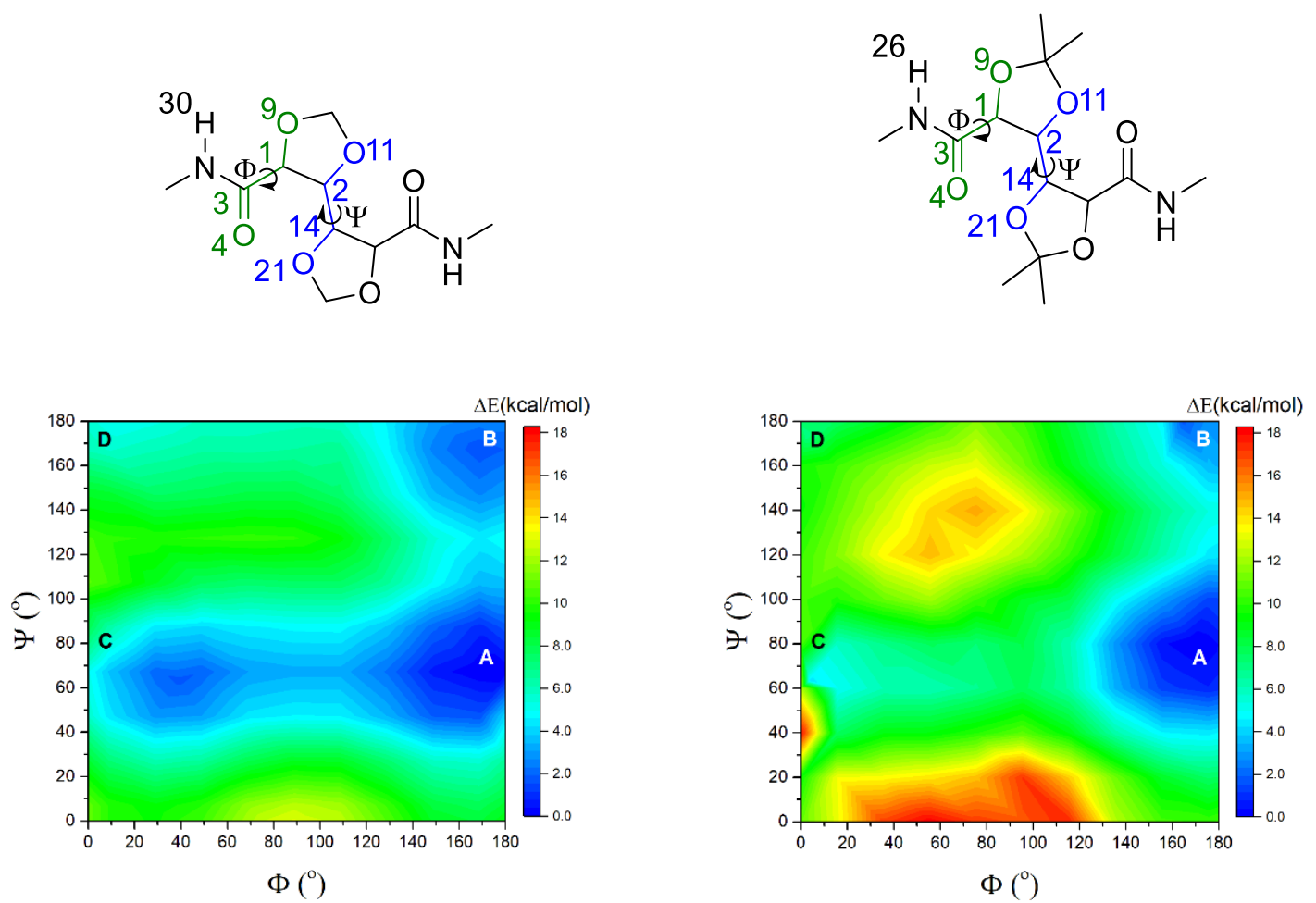

Figure 6-5 PES obtained by energy calculation and optimization of two GalX-NH-CH3 molecules: GalXH-NH$\mathrm{CH}_{3}$ (left) and $\mathrm{GalXMe}-\mathrm{NH}-\mathrm{CH}_{3}$ (right). The surfaces were calculated along two scanned dihedrals $\Phi$ (atoms $\mathrm{O}_{9}-\mathrm{C}_{1}-\mathrm{C}_{3}-\mathrm{O}_{4}$ ) and $\Psi$ (atoms $\mathrm{O}_{11}-\mathrm{C}_{2}-\mathrm{C}_{14}-\mathrm{O}_{21}$ ) from $0^{\circ}$ to $180^{\circ}$. Four located minima are labeled as $\mathrm{A}, \mathrm{B}, \mathrm{C}$ and $D$. The A structure is corresponding to the global minimum and $B, C, D$ to local minima.

The scan revealed that both GalX molecules have 4 minima in the investigated range of dihedral angles. The minima A and $\mathrm{C}$ correspond to $40-100^{\circ}$ angle $\Psi$ between the two acetal rings and minima $\mathrm{B}$ and $\mathrm{D}$ to angle $\Psi$ around $180^{\circ}$. Additionally, two minima (A and $\mathrm{B}$ ) have the dihedral $\Phi$ between carbonyl group and acetal ring around $180^{\circ}$ and the two other $(\mathrm{C}$ and D) around $40-0^{\circ}$. The A structure represents a global minimum whereas structures B, C and $\mathrm{D}$ are the local minima. In all presented structures amide hydrogen atom $\mathrm{H}_{30} / \mathrm{H}_{26}$ is located within a hydrogen-bond-formation distance to an oxygen atom within the molecule. The 


\section{Chapter 6}

distances between those two atoms are presented in Table 6-2. It is clear that both investigated GalX can adopt similar conformations; however, the distribution of conformers in the sample varies significantly. Based on the Boltzmann's formula, GalXH appears to have more stable conformers in the sample and the difference in energy between particular structures is lower than for GalXMe as supported by the respective wavenumbers of the hydrogen bonded $\mathrm{NH}$ stretch bands by FTIR - 3281 and $3329 \mathrm{~cm}^{-1}$ respectively. The rotational energy required for the transformation of one structure to another is much lower in case of the former one and increases significantly for GalXMe, most probably due to the sterical hindrance provided by the methyl groups on the isopropylidene acetal. Simultaneously, the presence of those additional groups influences the values of both dihedral angles of the optimized structures. For all located minima, the distance between a proton from N-H and oxygen falls within the hydrogen bond distance. The location of those four minima in this position is of high importance since it allows drawing the conclusion that the structures of the GalX amide are stabilized by the formation of intramolecular hydrogen bonds with one of the oxygen atoms (see Table 6-2 for the distances between proton $(\mathrm{NH})$ and oxygen $(\mathrm{O})$ ). If this is translated to a macromolecular system, like polyamide, this observation reveals lower affinity of amide protons to form interchain amide-amide hydrogen bonding and shows that indeed amide protons are engaged in local intramolecular $\mathrm{NH}$-acetal hydrogen bonding, which, in consequence, leads to the suppression of crystallinity.

Table 6-2 The calculated values of scanned coordinates for optimized structures of GalXH-NH-CH 3 and GalXMe-NH-CH3, energy of optimized structures relative to the global minimum energy, population of conformer and the distance between amide proton and the closest oxygen where it can form hydrogen bonds with.

\begin{tabular}{|c|c|c|c|c|c|c|c|}
\hline \multirow[t]{2}{*}{ Conformer } & \multirow[t]{2}{*}{$\Phi\left({ }^{\circ}\right)$} & \multirow[t]{2}{*}{$\Psi\left({ }^{\circ}\right)$} & \multirow{2}{*}{$\begin{array}{l}\Delta E^{a} \\
(\mathrm{kcal} / \mathrm{mol})\end{array}$} & \multirow{2}{*}{$\begin{array}{l}\text { Population }^{b} \\
\text { (\%) }\end{array}$} & \multicolumn{3}{|c|}{$L^{c}(A)$} \\
\hline & & & & & $\mathrm{NH}_{-} \mathrm{O}_{9}$ & $\mathrm{NH}_{-} \mathrm{O}_{21}$ & $\mathrm{NH}-\mathrm{O}_{16}$ \\
\hline \multicolumn{8}{|c|}{ GalXH-NH-CH3 } \\
\hline A & 179.6 & 67.2 & 0.00 & 87.89 & 2.204 & - & - \\
\hline B & 168.8 & 167.4 & 1.61 & 6.77 & 2.164 & - & - \\
\hline $\mathbf{C}$ & 39.5 & 63.5 & 1.76 & 5.32 & - & - & 2.142 \\
\hline D & 15.3 & 179.4 & 5.00 & 0.03 & - & 2.354 & - \\
\hline \multicolumn{8}{|c|}{ GalXMe- $\mathrm{NH}-\mathrm{CH}_{3}$} \\
\hline $\mathbf{A}$ & 168.9 & 80.6 & 0.00 & 94.26 & 2.129 & - & - \\
\hline B & 164.5 & 180.0 & 1.77 & 5.66 & 2.215 & - & - \\
\hline $\mathrm{C}$ & 3.7 & 63.5 & 4.42 & 0.08 & - & - & 2.114 \\
\hline D & 2.0 & 172.7 & 7.10 & 0.00 & - & 2.110 & - \\
\hline
\end{tabular}

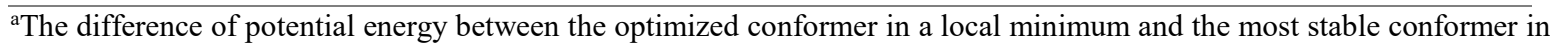
the global minimum. For global minimum $\Delta \mathrm{E}=0 \mathrm{kcal} \cdot \mathrm{mol}^{-1}$ and for local minimum $\Delta \mathrm{E}>0 \mathrm{kcal} \cdot \mathrm{mol}^{-1},{ }^{\mathrm{b}} \mathrm{Calculated}$ based on the potential energy of conformers in $\mathrm{mHa}(1 \mathrm{mHa}=627503 \mathrm{kcal} / \mathrm{mol})$ according to the Boltzmann's formula describing the ratio of populations of two states $\mathrm{i}$ and $\left.\mathrm{j}: \mathrm{N}_{\mathrm{j}} / \mathrm{N}_{\mathrm{i}}=\mathrm{EXP}\left(-\left(\varepsilon_{\mathrm{j}}-\varepsilon_{\mathrm{i}}\right) / \mathrm{kT}\right)\right), \varepsilon$ - energy of conformer in miliHaartree $(\mathrm{mHa}), \mathrm{kT} \sim 1 \mathrm{mHa}$ at room temperature, ${ }^{c}$ Calculated distance between amide hydrogen atom $\left(\mathrm{H}_{30}\right.$ for $\mathrm{GalXH}$ and $\mathrm{H}_{26}$ for $\left.\mathrm{GalXMe}\right)$ and the closest oxygen atom in the vicinity of this hydrogen atom. 
To visualize the hydrogen bonding formation by amide hydrogens, 4 optimized structures of GalXH-NH- $\mathrm{CH}_{3}$ are presented in Figure 6-6 (corresponding structures for GalXMe-NH- $\mathrm{CH}_{3}$ can be found in Figure D-1 in Appendix D). The hydrogen atoms $\mathrm{H}_{30}$ in two of the most stable conformers $\mathrm{A}$ and $\mathrm{B}$ form hydrogen bonding with oxygen on the neighboring acetal ring $\mathrm{O}_{9}$ whereas other conformers exist with the opposite carbonyl oxygen $\mathrm{O}_{16}$ (conformer $\mathrm{C}$ ) or the opposite acetal ring $\mathrm{O}_{21}$ (conformer $\mathrm{D}$ ).

A
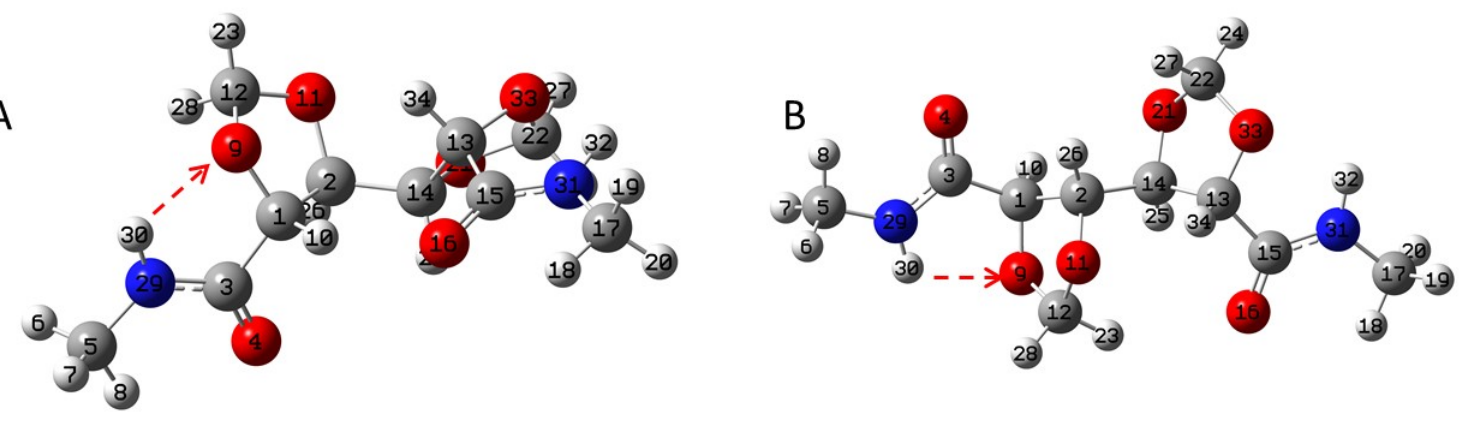

C
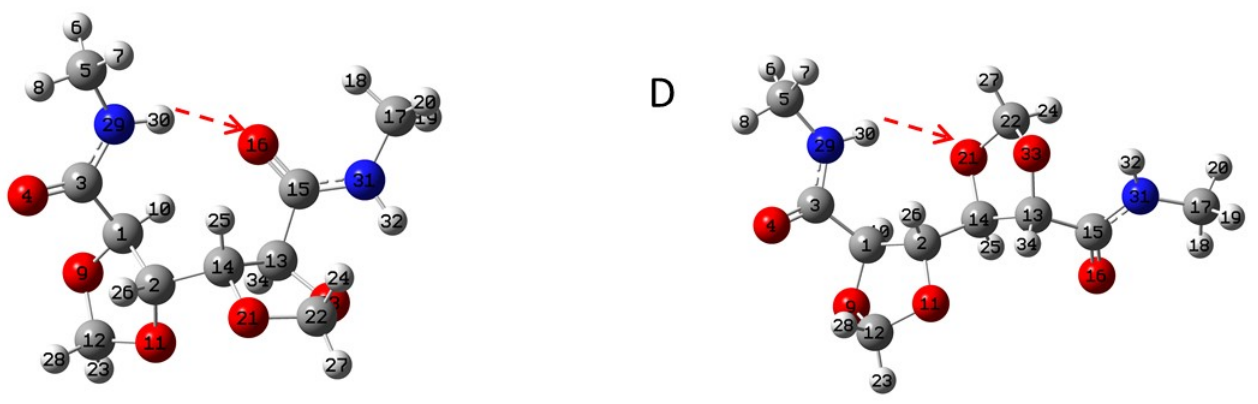

Figure 6-6 Optimized structures of conformers $\mathrm{A}, \mathrm{B}, \mathrm{C}$ and $\mathrm{D}$ of $\mathrm{GalXH}-\mathrm{NH}-\mathrm{CH}_{3}$ and the corresponding intramolecular hydrogen bond formation between hydrogen atom $\mathrm{H}_{30}$ and the oxygen atom in its vicinity.

The analysis of PES for the corresponding ester of both GalX molecules provides additional information. Both types of molecule, the amides (Figure 6-5) and the esters (Figure 6-7), possess minima located for similar values of scanned coordinates i.e. dihedral $\Psi$ and $\Phi$, however, the switch between two conformers requires less energy for esters than for amides (rotational barrier is lower for esters). Amide molecules are additionally stabilized in their minima by the formation of additional bonds; in this case those are formerly described intramolecular hydrogen bonds between amide protons and oxygens from acetal rings. 


\section{Chapter 6}
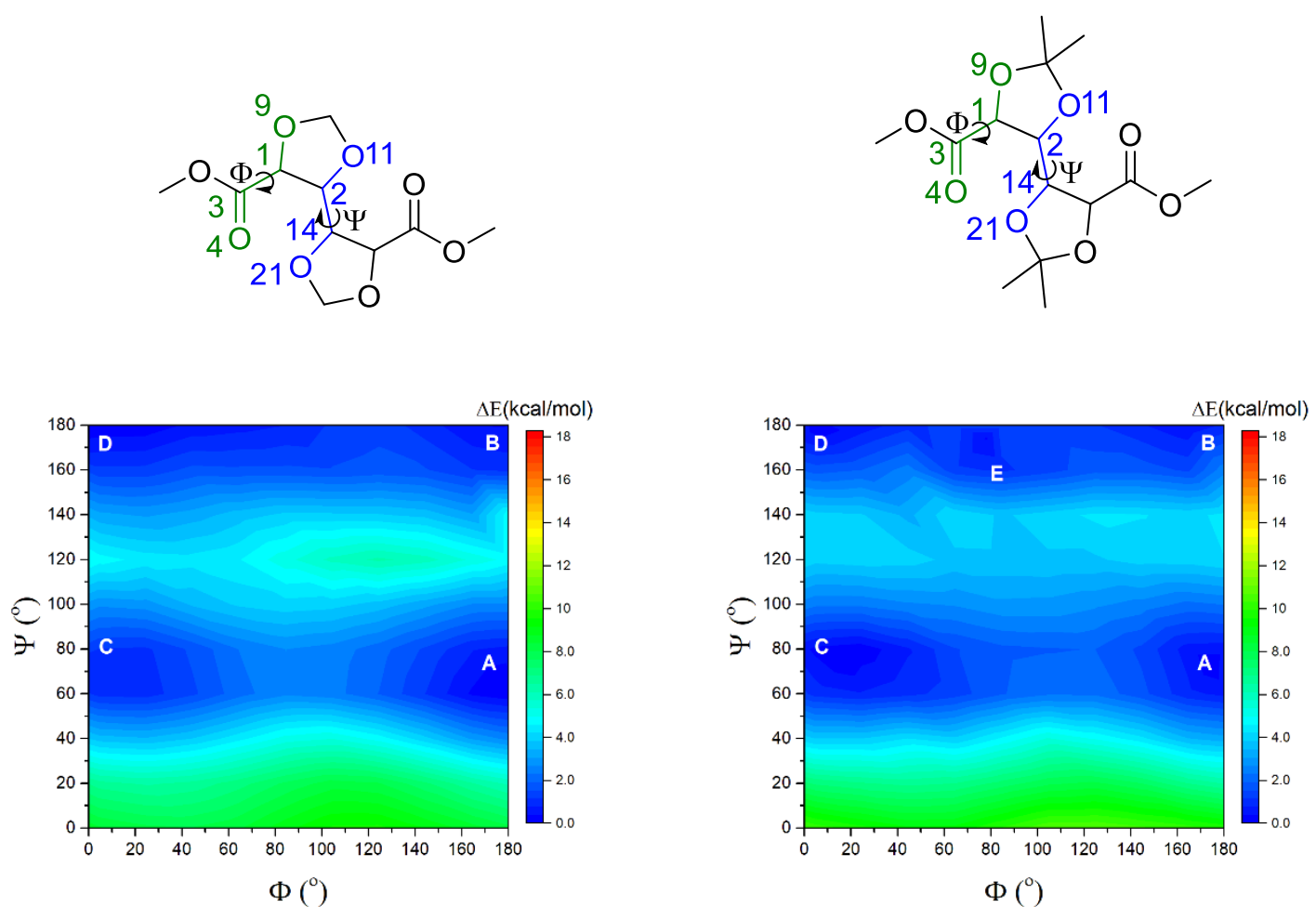

Figure 6-7 PES obtained by energy calculation and optimization of two GalX-OCH $\mathrm{H}_{3}$ molecules: GalXH-OCH (left) and $\mathrm{GalXMe}_{\mathrm{X}} \mathrm{OCH}_{3}$ (right). The surfaces were calculated along two scanned dihedrals $\Phi$ (atoms $\mathrm{O}_{9}-\mathrm{C}_{1^{-}}$ $\mathrm{C}_{3}-\mathrm{O}_{4}$ ) and $\Psi$ (atoms $\mathrm{O}_{11}-\mathrm{C}_{2}-\mathrm{C}_{14-} \mathrm{O}_{21}$ ) from $0^{\circ}$ to $180^{\circ}$. The located minima are labeled as $A, B, C, D$ and $E$.

\subsection{Distribution of GalX between the amorphous and crystalline phase of copolymers}

To evaluate further the influence of GalXH and GalXMe on the interactions in the polymer, a series of semicrystalline polyamides were prepared (co)PA1-PA9 with varied amount of GalX incorporated into the structure (see Table 6-1).

It was previously revealed that GalX highly suppresses the crystallinity upon the incorporation in copolyamides (see Chapter 4). To elucidate the effect of the cyclic moieties on thermally induced conformational changes in polyamides in the solid state and in the melt, temperature dependent FT-IR (tdFT-IR) was recorded (Figure 6-8). 

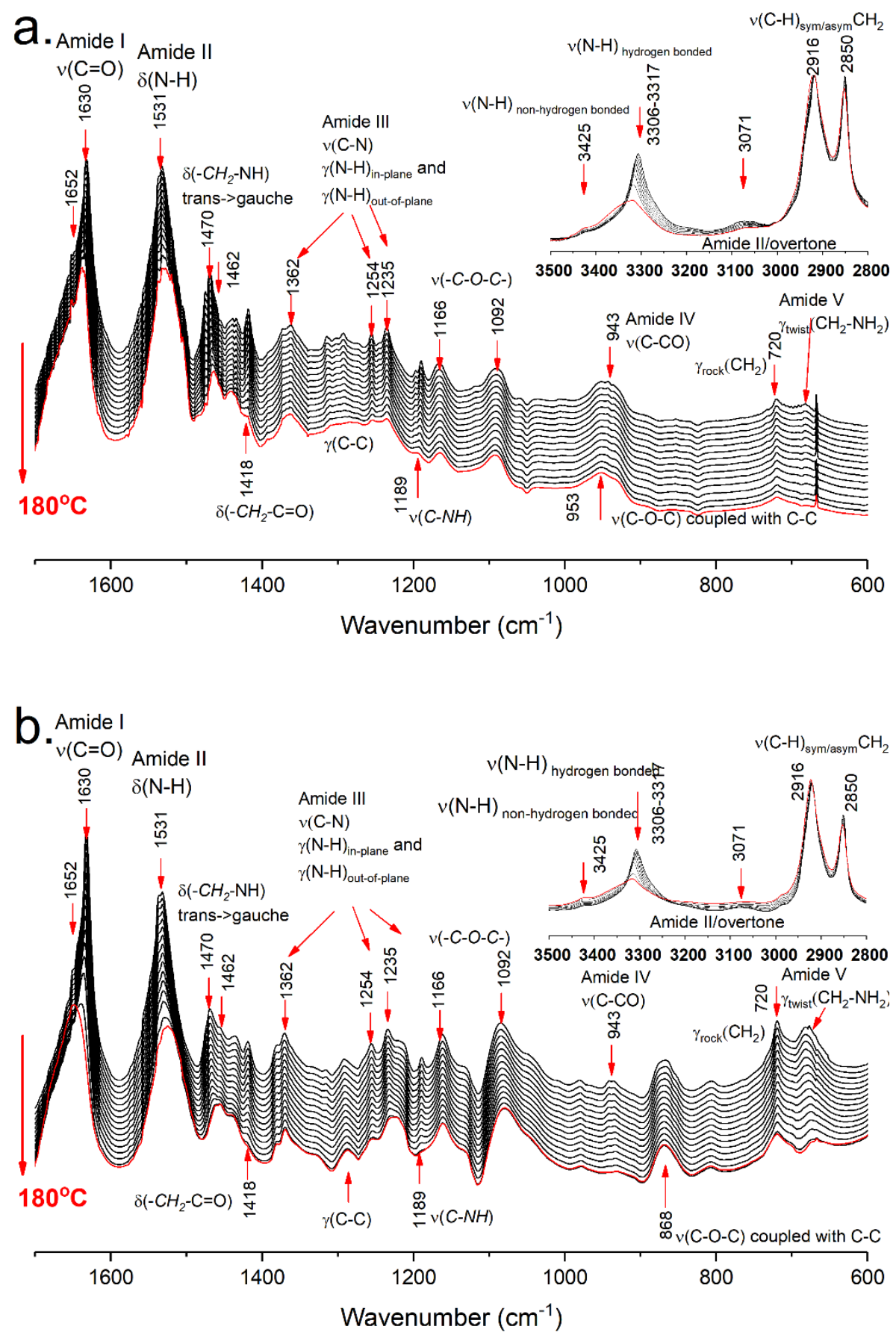

Figure 6-8 Temperature dependent FT-IR spectrum recorded for two copolyamides (a) $P A\left(G_{\text {GalXH }} 7, D D D A_{105}, S_{73}\right) c o P A 3$ with $\mathrm{T}_{\mathrm{m}}=170^{\circ} \mathrm{C}$ and $\Delta \mathrm{H}_{\mathrm{m}}=46 \mathrm{~J} / \mathrm{g}$ based on the $2^{\text {nd }}$ heating cycle DSC and (b) $P A\left(G_{\text {GalXMe }}{ }_{24}, D D D A_{105}, S A_{76}\right) \operatorname{co} P A 7$ with $\mathrm{T}_{\mathrm{m}}=169^{\circ} \mathrm{C}$ and $\Delta \mathrm{H}_{\mathrm{m}}=43 \mathrm{~J} / \mathrm{g}$ based on the $2^{\text {nd }}$ heating cycle DSC. $\delta$ - bending (scissoring), $\gamma$-deformation/backbone vibration (rock-rocking, twist-twisting, wag - wagging), $v$ - stretching vibration.

The shape and intensities of particular peaks on the IR spectrum as a function of temperature provide information about the packing (e.g. $\gamma_{\text {rock }}\left(\mathrm{CH}_{2}\right)$ most-in-phase rocking vibration characteristic for triclinic packing of n-alkanes), hydrogen bonding (e.g stretching vibration of hydrogen bonded $\mathrm{N}-\mathrm{H}$ at lower wavenumbers than the corresponding wavenumber for 


\section{Chapter 6}

non-hydrogen bonded N-H), crystal perfection, transitions e.g. Brill transition ${ }^{27}$ of polyamides or content of particular phases e.g. amorphous/crystalline. The more bulky structure of cyclic moieties in comparison to aliphatic components influences the presence and amount of weakly-hydrogen bonded N-H groups. In the investigated polyamides the stretching mode of weakly-associated $(\mathrm{N}-\mathrm{H})$ at $3425 \mathrm{~cm}^{-1}$ is present already at room temperature whereas typically for highly crystalline aliphatic polyamides it appears above the melting point. ${ }^{25}$ Due to the weakening of hydrogen bonds with increase of temperature the peak of strongly associated N-H groups at $3306 \mathrm{~cm}^{-1}$ is broadening and shifts towards higher wavenumber values $\left(3317 \mathrm{~cm}^{-1}\right)$. A similar phenomenon is observed for Amide I (stretching vibration $v(\mathrm{C}=\mathrm{O})$ at $1630 \mathrm{~cm}^{-1}$ shifts towards higher wavenumbers) and Amide II (scissoring $\delta(\mathrm{N}-\mathrm{H})$ at $1530 \mathrm{~cm}^{-1}$ shifts towards lower wavenumbers) bands as a function of increasing temperatures. The spectral region corresponding to conformational changes of the methylene segments, e.g. trans and and/or gauche, is between 1470 and $1418 \mathrm{~cm}^{-1}$. The peak at $1470 \mathrm{~cm}^{-}$ ${ }^{1}$ (trans) shifts to $1462 \mathrm{~cm}^{-1}$ (gauche), which is related to the conformational changes associated with scissoring vibrations of a methylene unit connected to an $\mathrm{NH}$ group. ${ }^{25}$ Simultaneously the peak at $1418 \mathrm{~cm}^{-1}$ originating from the corresponding mode of the methylene unit connected to $\mathrm{C}=\mathrm{O}$ shifts towards higher values. A significant difference in both spectra is visible in the region of Amide IV vibrations. On the spectrum of GalXH copolyamide (Figure 6-8, (a)) signal related to the Amide IV mode (stretching vibration of $(\mathrm{C}-\mathrm{C}=\mathrm{O}))$ is present at $953 \mathrm{~cm}^{-1}$. A stretching vibration of $(\mathrm{C}-\mathrm{O}-\mathrm{C})$ coupled with $(\mathrm{C}-\mathrm{C})$ is present at $943 \mathrm{~cm}^{-1}$. Those two signals are strongly overlapping on the GalXH spectrum, however on the spectrum of the GalXMe copolymer (Figure 6-8, (b)) there are two peaks present in this region at 943 and $868 \mathrm{~cm}^{-1}$. This change is a very distinguishable difference between polyamides from those two monomers.

\subsection{Hydrogen bonding efficiency}

Investigation of hydrogen bonding in homopolyamides and melting behavior of copolyamides indicates that the cooperative hydrogen bonding efficiency is affected by the increasing concentration of GalX moieties in a polymer (Figure 6-9). The FT-IR spectra recorded for the prepared (co)polyamides PA1, coPA2-PA4 and coPA6-PA8 show the progressive evolution of the non-hydrogen bonded, broad stretching vibration of $\mathrm{N}-\mathrm{H}$. Initially, for homopolyamide consisting of sebacic acid and 1,12-dodecanedimine (PA1), the peak is sharp and narrow. The visible transformation of the peak occurs above $8-9$ mol\% 
GalX incorporated into the polyamide structure, at which point the peak starts to broaden as it is affected by a decreasing hydrogen bonding efficiency. The evolution of this range of spectra showed that the changes are more pronounced for the series with GalXMe (coPA5coPA9) and higher concentration of GalX moieties eventually leads to the existence of a fully resolved additional peak corresponding to non-hydrogen bonded $\mathrm{N}-\mathrm{H}$ (above $3400 \mathrm{~cm}^{-1}$ ) already at room temperature.

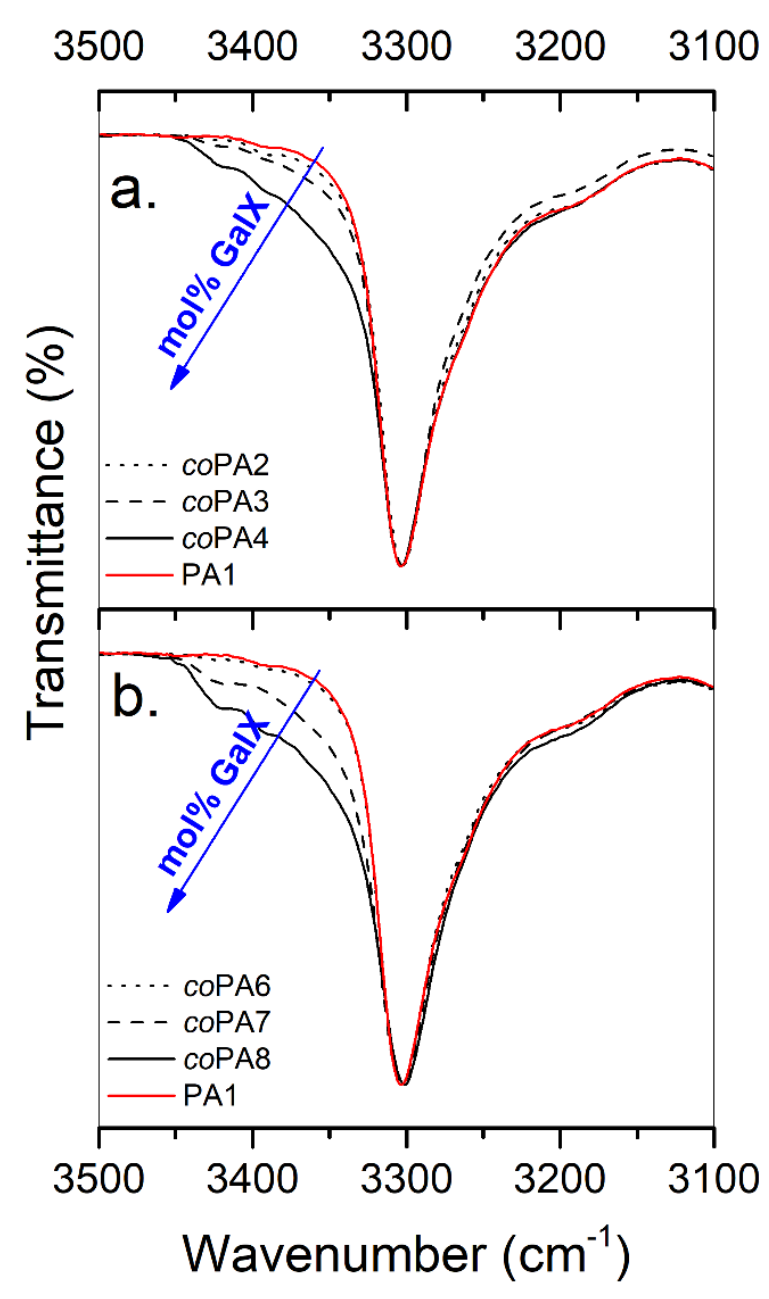

Figure 6-9 The representation of the hydrogen bonding efficiency evolution (transformation of $N$ - $H$ stretching vibration) triggered by the introduction of the increasing amount of GalX moieties to homopolyamide $P A\left(D D D A_{93}, S_{100}\right)(P A 1)$. (a) GalXH copolymer series coPA2-coPA4 and (b) GalXMe copolymer series coPA6-соPA9.

\subsection{Melting behavior and Wide-Angle X-ray Diffraction.}

WAXD and the melting behavior of (co)polyamides were studied on films casted from a HFIP solution to facilitate optimum chain packing and emplify heat flux changes corresponding to the crystalline phase. 


\section{Chapter 6}

Table 6-1 lists the chemical composition, melting temperatures and crystallinity of all (co)polyamides as collected by DSC and WAXD. In general, it is widely recognized that the incorporation of bulky cyclic moieties lowers crystallinity of polymers and may lower the melting point assuming that the concentration of cyclic comonomer is sufficient, which is also applicable in this case. ${ }^{28}$ Additionally, aliphatic reference polyamide PA(DDDA,SA) shows a double melting peak of which the first was assigned to reorganization of relatively thin to thicker lamellar crystals upon heating prior to complete melting and the second to an endothermic event ${ }^{29}$. In order to distinguish heat effects of crystallographic reorganizations and true melting, DSC measurements were carried out at heating rates of 1 and $10^{\circ} \mathrm{C} / \mathrm{min}$. Independent of the chemical composition the comparison of the DSC thermograms recorded at 1 and $10^{\circ} \mathrm{C} / \mathrm{min}$ reveals that the first endothermic event is less heating rate dependent than the second endothermic event (Figure 6-10).
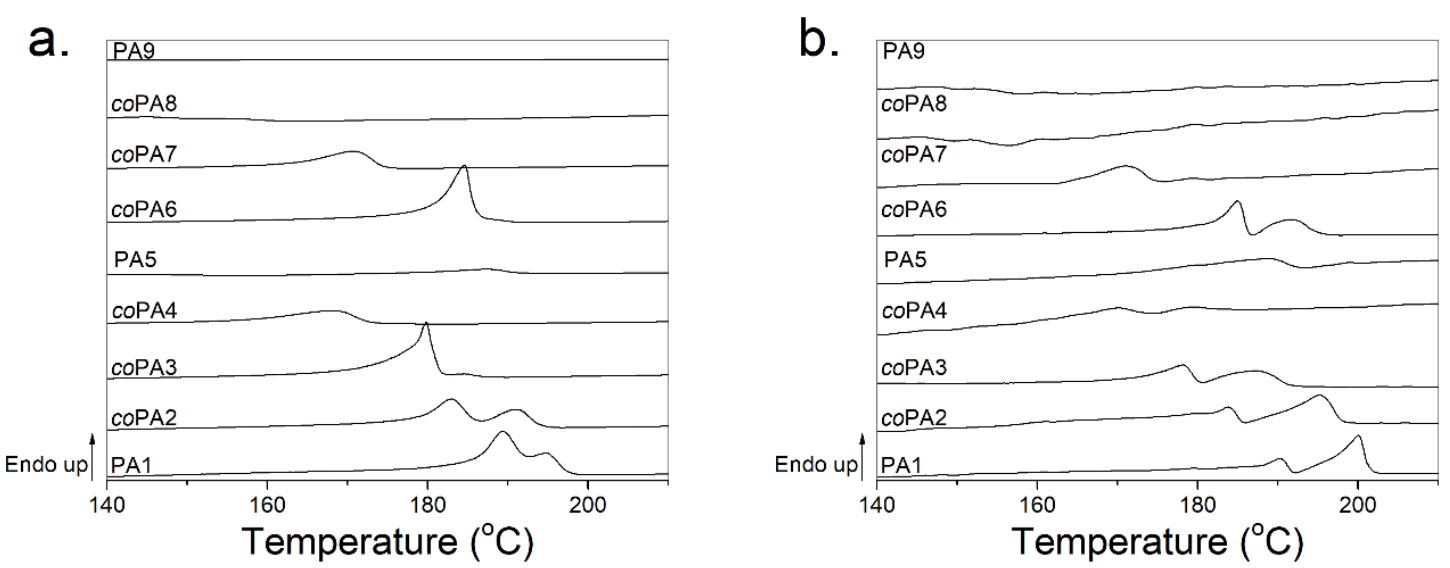

Figure 6-10 The melting profiles of (co)PA1-(co)PA9 collected at heating rate a. $10^{\circ} \mathrm{C} / \mathrm{min}$ and $\mathrm{b} .1^{\circ} \mathrm{C} / \mathrm{min}$ from the first heating cycle of a casted polyamide film.

The small exothermic event that succeeds the first endothermic event, as witnessed at $1{ }^{\circ} \mathrm{C} / \min$ (Figure 6-10(b)), also supports the reorganization of thin lamellar crystals for both the GalXH and GalXMe copolymers of this study. As a low heating rate provides more time for reorganization, the crystalline phase further increases the lamellar thickness and so the melting temperatures. Due to the overlapping heat effects, standard DSC cannot be used to evaluate the melting enthalpy and calculate crystallinity. However, two distinct effects on the incorporation of GalX comonomers are apparent. Firstly, as expected from perturbed chain packing and regardless potential reorganization, increasing the GalX comonomer content decreases the melting temperatures and enthalpies. Secondly, at relatively high comonomer concentrations reorganization prior to eventual melting is no longer observed. This is likely caused by the bulky nature and limited rotational freedom of the GalX comomoners. In fact, 
especially for GalXMe, reorganization is only witnessed at $1{ }^{\circ} \mathrm{C} / \mathrm{min}$ and the coPA6 and coPA7 samples. The incorporation of stiff chain segments in a crystalline lattice induces an increase in melting point by reducing the change in entropy, i.e. the number of conformations, upon melting. Since for all copolymers both the melting temperatures and enthalpies lower upon increasing the comonomer content, it is unlikely that the comonomers are included with the crystalline lattice.

The decrease in crystallinity with increased GalX comonomer content was supported by WAXD analysis listed in Table 6-1. Furthermore, potential incorporation of the GalX comonomers within the crystal lattice will affect chain packing and thus lattice parameters. Wide-angle diffraction patterns for the synthesized copolymers with a systematic, progressive increase in GalXH and GalXMe, Figure 6-11, only reveal a correlated decrease in intensity (crystallinity) and no changes in the scattering angle $2 \theta$ and thus lattices.

a.

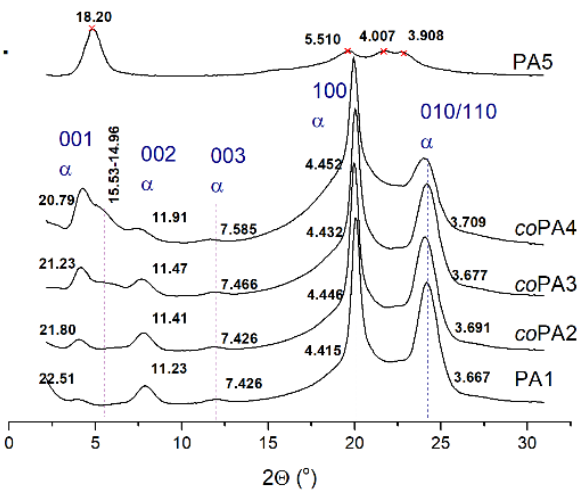

b.

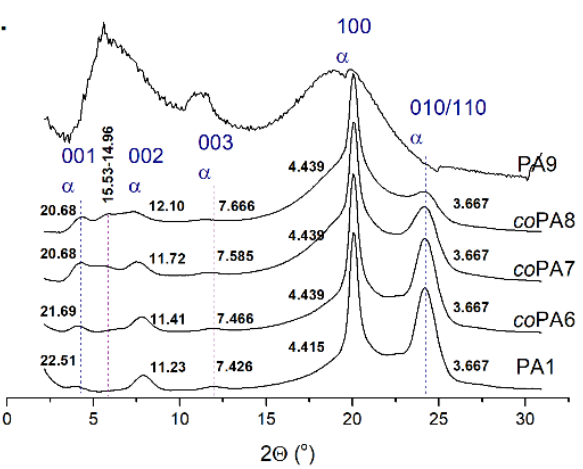

Figure 6-11 X-ray diffraction patterns of polyamide films with different content of the GalX monomer, casted from an HFIP solution $(1 \mathrm{~g} / 10 \mathrm{~mL}$ ) and measured at room temperature for (a) polyamides based on GalXH and (b) polyamides based on GalXMe. The d-spacing (A) of the reflections corresponding to the a phase of the of polyamide are shown as numbers on the profile.

\subsection{Conclusions}

FT-IR studies showed that upon the incorporation of GalX motifs into aliphatic polyamides the efficiency of intermolecular hydrogen bonding decreases and the corresponding peak on the FT-IR spectrum (stretching N-H vibration) becomes broad and diffused. This can be related to the fact that GalX amides adopt configurations, which allow the formation of intramolecular hydrogen bonding. The GalX esters do not seem to show a similar trend due to the lack of an amide proton, which can form the hydrogen bond and thus stabilize this particular conformation of the molecule. A typical intermolecular hydrogen bonding network observed for aliphatic polyamides is disrupted by the presence of the acetal motifs in GalX 


\section{Chapter 6}

polyamides, in particular the presence of the oxygen on the $\alpha$ carbon which forms the hydrogen bond with the neighboring amide proton.

The incorporation of GalX motifs into copolyamides perturbes the chain packing of such materials leading to the decrease in melting point. The reorganization of thin lamellar crystals upon heating, typically observed above the melting temperature, is suppressed due to the rigidity of the monomers, which is more pronounced for GalXMe. For all copolymers the melting temperatures and enthalpies lower upon increasing the comonomer content and thus it is unlikely that the comonomers are included in the crystalline lattice.

\subsection{Materials and Methods}

\subsubsection{Materials}

2,3:4,5-di- $O$-isopropylidene-galactaric acid $>99 \%$, and 2,3:4,5-O-dimethylene-galactaric acid $>99 \%$, diethyl 2,3:4,5-di- $O$-isopropylidene-galactarate $>99 \%$, diethyl 2,3:4,5-di-Omethylene-galactarate $>99 \%$, were supplied by Royal Cosun. 1,6-hexamethylenediamine (C6HMDA) 98\%, 1,12-dodecanediamine (DDDA-12) 98\%, decanedioic acid (SA) 98\%, sodium hypophosphite monohydrate $>99 \%\left(\mathrm{NaHPO}_{3} \cdot \mathrm{H}_{2} \mathrm{O}\right)$ were purchased from Sigma-Aldrich and used as supplied. 1,1,1,3,3,3-hexafluoro-2-propanol (HFIP) was purchased from Acros Organics. Acetone was purchased from Biosolve.

\subsubsection{Methods}

Synthesis of homopolyamides poly(hexamethylene-2,3:4,5-O-isopropylidene-galactaramide and poly(hexamethylene-2,3:4,5-O-methylene-galactaramide was conducted according to the procedure described in Chapter 2.

Synthesis of copolyamides Poly(1,6-diaminohexane-1,12-dodecanedioate)-co-poly(1,6diaminohexane-2,3:4,5-O-isopropylidene-galactarate) and poly(1,6-diaminohexane-1,12dodecanedioate)-co-(1,6-diaminohexane-2,3:4,5-O-methylene-galactarate) was conducted according to the procedure described in Chapter 4.

\subsubsection{Characterization.}

Molecular weights determined by GPC was determined as described in the experimental section in Chapter 2.

Potential energy surfaces (PES) were calculated using the density functional theory (DFT) method with diffusion functions on heavy atoms and polarization functions on hydrogen 
using the B3-LYP/6-31G (d,p) basis set with Gaussian 09 and GaussView 05 as software package $^{30}$. Conformational analysis was performed by scanning two dihedral angles i.e. $\Phi$ and $\Psi$ from $0^{\circ}$ to $180^{\circ}$ with optimization and frequency calculations in each step. The geometries in all located minima were additionally re-evaluated using the same level of theory to achieve correct angles and interatomic distances. Potential energy values were normalized towards the global minimum and expressed in $\mathrm{kcal} / \mathrm{mol}$. The population of conformers was calculated using Boltzman's formula describing the ratio of populations of two states $\mathrm{i}$ and $\left.\mathrm{j}: \mathrm{N}_{\mathrm{j}} / \mathrm{N}_{\mathrm{i}}=\mathrm{EXP}\left(-\left(\varepsilon_{\mathrm{j}}-\varepsilon_{\mathrm{i}}\right) / \mathrm{kT}\right)\right), \varepsilon$ - energy of conformer in miliHartree $(\mathrm{mHa})$, $\mathrm{kT} \sim 1 \mathrm{mHa}$ at room temperature. ${ }^{31}$ The calculations were conducted on an $\operatorname{Intel}{ }^{\circledR} \mathrm{Xeon}{ }^{\circledR}$ workstation equipped with CPU E5-2650 v4, $2.2 \mathrm{GHz}$ and $32 \mathrm{~GB}$ ram memory.

Copolyamide films were prepared by dissolving around $1 \mathrm{~g}$ of a polymer in $10 \mathrm{~mL}$ HFIP. Upon full dissolution the mixture was placed in Teflon coated crystallization dishes and left overnight under the fumehood for HFIP to evaporate. The teflon petri dish was covered with another petri dish to ensure slow evaporation of the solvent. This procedure resulted in a thin polyamide film.

The thermal profiles were recorded using differential scanning calorimetry (DSC) on a Netzsch Polyma 2014 DSC. DSC data was obtained from about 2-5 mg of polymer film at heating/cooling rates of $1^{\circ} \mathrm{C} / \mathrm{min}^{-1}$ and $10{ }^{\circ} \mathrm{C} \mathrm{min}{ }^{-1}$ under a nitrogen flow of $20 \mathrm{~mL} \mathrm{~min}{ }^{-1}$. Indium, zinc, tin and bismuth were used as standards for temperature and enthalpy calibration. DSC heating and cooling cycles were performed from 25 to $250{ }^{\circ} \mathrm{C}$. The reported melting temperatures and the enthalpy values of polyamide films correspond to the first heating cycle.

Infrared spectroscopy (FTIR) and temperature - dependent infrared spectroscopy (td-FTIR) was recorded on a PerkinElmer FTIR/NIR spectrometer Frontier with resolution $4 \mathrm{~cm}^{-1}$ and 8 accumulations per spectrum. A polyamide powder was melted on the FT-IR crystal and slowly cooled down to RT. Subsequently, such a sample was heated from $40{ }^{\circ} \mathrm{C}$ to $180{ }^{\circ} \mathrm{C}$ with a heating rate of $10^{\circ} \mathrm{C} / \mathrm{min}$ and spectra were recorded at temperature intervals of $10^{\circ} \mathrm{C}$. The background scan was recorded at $180{ }^{\circ} \mathrm{C}$ with 64 accumulations and resolution $4 \mathrm{~cm}^{-1}$. Results were normalized to the stretch vibration of the methylene group at $2920 \mathrm{~cm}^{-1}$. All data were processed using the Spectrum (PerkinElmer) software package and SpectraGryph 1.0.3.

Wide-Angle X-ray Diffraction (WAXD) measurements were carried out using a measurements were carried out using a Ganesha SAXS/WAXS 300XL at room temperature on samples 


\section{Chapter 6}

casted from HFIP solution with monochromatic source of radiation $\mathrm{Cu} K \alpha(\lambda=1.5418 \AA)$ and detector PILATUS 300K. The obtained patterns were integrated and subsequently $1 \mathrm{D}$ profiles were obtained. The profiles were used to calculate the degree of crystallinity by the substraction of an amorphous halo from the crystalline peaks 100 and $010 / 110$ of PA(DDDA,SA) as demonstrated in Figure D-2 in the Appendix D. 


\section{REFERENCES}

1. C. Lavilla and S. Muñoz-Guerra, Green Chem., 2013, 15, 144-151.

2. E. Zakharova, A. Martínez de Ilarduya, S. Leon and S. Muñoz-Guerra, Des. Monomers Polym., 2017, 20, 157-166.

3. A. A. Wróblewska, J. Noordijk, N. Das, C. Gerards, S. M. A. Wildeman and K. V. Bernaerts, Macromol. Rapid Commun., 2018, 34, 1800077.

4. C. Lavilla, A. Alla, A. Martínez de Ilarduya, E. Benito, M. G. García-Martín, J. A. Galbis and S. Muñoz-Guerra, J. Polym. Sci., Part A: Polym. Chem., 2012, 50, 1591-1604.

5. $\quad$ S. Munoz-Guerra, High Perform. Polym., 2012, 24, 9-23.

6. A. A. Wróblewska, K. V. Bernaerts and S. M. A. De Wildeman, Polymer, 2017, 124, 252262.

7. P. Ruiz-Donaire, J. J. Bou, S. Munoz-Guerra and A. Rodriquez-Galan, J. Appl. Polym. Sci., 1995, 58, 41-54.

8. C. Lavilla and S. Muñoz-Guerra, Polym. Degrad. Stab., 2012, 97, 1762-1771.

9. M. E. Rogers and T. E. Long, Synthetic methods in step-growth polymers., A John Wiley \& Sons, inc., Hoboken, New Jersey, 2003.

10. E. Hellmann, J. Malluche and G. P. Hellmann, Polym. Eng. Sci., 2007, 47, 1600-1609.

11. J. Malluche, G. P. Hellmann, M. Hewel and H.-J. Liedloff, Polym. Eng. Sci., 2007, 47, 15891599.

12. R. Lazeroms, H. Raaijmaakers, C. E. Koning, A. Papegaai and A. Urnianova, WO 201S/074926 A1, 2018.

13. S. Muñoz-Guerra, C. Lavilla, C. Japu and A. Martínez de Ilarduya, Green Chem., 2014, 16, 1716-1739.

14. M. K. Dowd, D. E. Kiely and J. Zhang, Carbohydr Res, 2011, 346, 1140-1148.

15. J. M. Brown, M. Manley-Harris, R. J. Field and D. E. Kiely, J. Carbohydr. Chem., 2007, 26, 455-467.

16. B. P. Jarman, D. E. Kiely, M. Manley-Harris and B. K. Nicholson, J. Carbohydr. Chem., 2009, 28, 107-123.

17. D. E. Kiely, A. Vishwanathan, B. P. Jarman and M. Manley-Harris, J. Carbohydr. Chem., 2009, 28, 348-368.

18. B. Begines, F. Zamora, E. Benito, M. de Gracia García-Martín and J. A. Galbis, J. Polym. Sci., Part A: Polym. Chem., 2012, 50, 4638-4646.

19. C. Hahn, H. Keul and M. Möller, Polym. Int., 2012, 61, 1048-1060.

20. T.-M. Chen, Y.-F. Wang, S. Sakamoto, K. Okada and T. Nakaya, Des. Monomers Polym., 1998, 1, 447-465.

21. J. Wu, L. Jasinska-Walc, D. Dudenko, A. Rozanski, M. R. Hansen, D. van Es and C. E. Koning, Macromolecules, 2012, 45, 9333-9346.

22. L. Jasinska, M. Villani, J. Wu, D. van Es, E. Klop, S. Rastogi and C. E. Koning, Macromolecules, 2011, 44, 3458-3466.

23. L. Jasinska-Walc, D. Dudenko, A. Rozanski, S. Thiyagarajan, P. Sowinski, D. van Es, J. Shu, M. R. Hansen and C. E. Koning, Macromolecules, 2012, 45, 5653-5666.

24. A. Wroblewska, A. Zych, S. Thiyagarajan, D. Dudenko, D. van Es, M. R. Hansen, C. Koning, R. Duchateau and L. Jasinska-Walc, Polym. Chem., 2015, 6, 4133-4143.

25. X. Cui and D. Yan, J. Polym. Sci., Part B: Polym. Phys., 2004, 42, 4017-4022.

26. J. A. W. Harings, O. van Asselen, R. Graf, R. Broos and S. Rastogi, Crystal Growth \& Design, 2008, 8, 3323-3334.

27. Y. Yoshioka and K. Tashiro, Polymer, 2003, 44, 7007-7019.

28. C. Lavilla, E. Gubbels, A. Martínez de Ilarduya, B. A. J. Noordover, C. E. Koning and S. Muñoz-Guerra, Macromolecules, 2013, 46, 4335-4345.

29. L. Franco and J. Puiggalí, J. Polym. Sci., Part B: Polym. Phys., 1995, 33, 2065-2073.

30. Gaussian 09, Revision E.01, M. J. Frisch, G. W. Trucks, H. B. Schlegel, G. E. Scuseria, M. A. Robb, J. R. Cheeseman, G. Scalmani, V. Barone, B. Mennucci, G. A. Petersson, H. Nakatsuji, M. Caricato, X. Li, H. P. Hratchian, A. F. Izmaylov, J. Bloino, G. Zheng, J. L. 


\section{Chapter 6}

Sonnenberg, M. Hada, M. Ehara, K. Toyota, R. Fukuda, J. Hasegawa, M. Ishida, T. Nakajima, Y. Honda, O. Kitao, H. Nakai, T. Vreven, J. A. Montgomery, J. E. Peralta, F. Ogliaro, M. Bearpark, J. J. Heyd, E. Brothers, K. N. Kudin, V. N. Staroverov, T. Keith, R. Kobayashi, J. Normand, K. Raghavachari, A. Rendell, J. C. Burant, S. S. Iyengar, J. Tomasi, M. Cossi, N. Rega, J. M. Millam, M. Klene, J. E. Knox, J. B. Cross, V. Bakken, C. Adamo, J. Jaramillo, R. Gomperts, R. E. Stratmann, O. Yazyev, A. J. Austin, R. Cammi, C. Pomelli, J. W. Ochterski, R. L. Martin, K. Morokuma, V. G. Zakrzewski, G. A. Voth, P. Salvador, J. J. Dannenberg, S. Dapprich, A. D. Daniels, O. Farkas, J. B. Foresman, J. V. Ortiz, J. Cioslowski and D. J. Fox, Gaussian, Inc., Wallingford CT, 2013

31. J. B. Foresman and A. Frisch, Exploring Chemistry with Electronic Structure Methods, Gaussian, Inc., Wallingford, CT USA, 3rd edn., 2015. 


\section{Chapter 7 GALX POLYAMIDE BLENDS}

Abstract

This chapter focuses on thermal processing of 2,3:4,5-di-O-isopropylidene-galactaratebased polyamides. Three polyamides, namely GalXMe with m-xylenediamine (aliphatic/aromatic), isophoronediamine (cycloaliphatic) and p-phenylenediamie (aromatic), are blended with one semicrystalline and one amorphous polyamide using a twin screw extruder at temperatures above $260^{\circ} \mathrm{C}$. Biobased PA blends show limited to no miscibility with other polyamides; however, their incorporation results in strong materials with high Young's moduli. Investigation into the mechanism of blending revealed that for PA(PPDA,GalXMe) a transamidation reaction improved blend compatibility. The thermal stability of the biobased PAs depends on which diamine is used. Polymers with aliphatic/aromatic or alicyclic diamines show no degradation, whereas with fully aromatic diamines, such as p-phenylenediamine, some degradation processes are observed under extrusion conditions. 



\subsection{Introduction}

Similarly as for bio-based poly(lactic acid) (PLA), the mechanical performance of polymers containing cyclic sugar-derived moieties needs to be further improved. PLA is an extensively-researched, bio-based polyester with high potential for wide-spread applications. However, it possesses poor ductility, with an elongation at break only around $3 \%$. $^{1-3}$

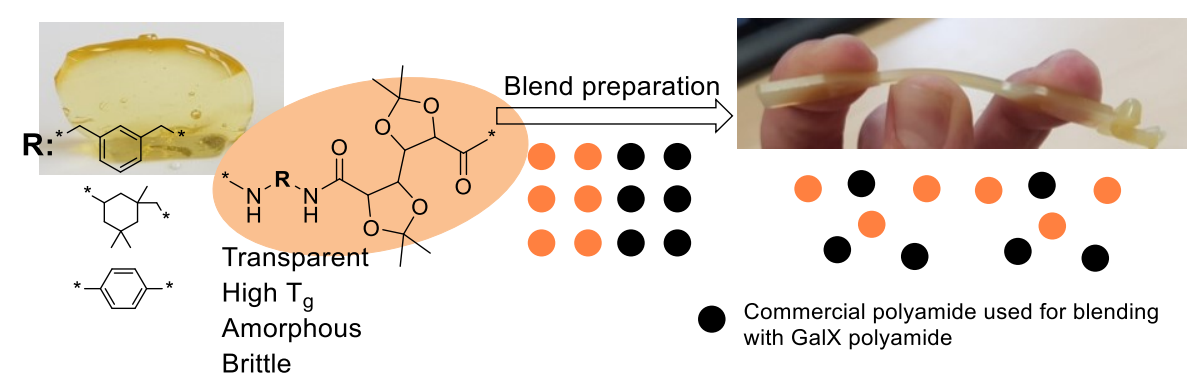

Figure 7-1 Approach towards the preparation of blends from GalX polyamides

There are several ways to improve the performance of bio-based materials: copolymerization $^{4-8}$, blending and/or reactive blending ${ }^{1,9-13}$, or by modification through the addition of additives (e.g. plasticizers or impact modifiers). ${ }^{14,15}$

The most common combination for carbohydrate-based polymers is the synthesis of copolyesters of biacetalized galactaric acid derivatives with aromatic and aliphatic diols $\mathrm{s}^{4,5,7}$, 16-22, and more recently GalX (co)polyamides. ${ }^{23,24}$ The conducted research demonstrates that the successful copolymerization gives a polyester material with improved properties (i.e. higher elongation at break) than pure carbohydrate-based polymers. ${ }^{5}$

Another approach to increase the elongation (at break) and strength of the polymeric materials is blending and/or reactive blending. A vast amount of research has been conducted on reactive blending of PLA with different polyesters, such as poly( $\omega$-hydroxytetradecanoic) $\operatorname{acid}^{1}, \operatorname{poly}(\varepsilon \text {-caprolactone })^{25}$, poly(butylene succinate $)^{26}$, or with copolyesters like poly(butylene adipate-co-terephthalate) ${ }^{27,} 28$ or poly(butylene succinate-co-adipate). ${ }^{28}$ Blending, in general, has numerous advantages over other methods of modification as it is considered cheap and industrially relevant. However, in case of blending the miscibility of the two components plays a predominant role in order to obtain blends with improved mechanical properties compared to the separate homopolymers. 


\section{Chapter 7}

While there is a significant amount of research conducted on PLA-based materials, the exact opposite is true for GalX-based polymers. This study aims at improving of properties of carbohydrate-based polymers, which could form the basis for material development thereof. Such polymers deserve significant attention as they can offer a wide variety of advantageous properties like transparency or thermal properties for material design. However, as with all polymeric materials at the beginning of their development, broad investigation is required. As such, it is proposed to blend GalX-based amorphous homopolymers with two different commercially available polyamides to improve the thermal properties of the latter, and the mechanical properties (improve brittleness) of the former. The polymers will be mixed in an extruder at elevated temperatures and the properties of the blends will be evaluated.

\subsection{Preparation of blends}

Three GalX polymers were chosen to be blended with commercial polyamides, namelysemicrystalline PA(HMDA,DA) and Trogamid CX9704. The latter is an amorphous, transparent polyamide with high glass transition temperature composed of PACM and DA. All GalX polymers are amorphous, transparent materials and the chemical characteristics of the used polymers are summarized in Figure 7-2.The GalX PAs chosen for the blending experiments differ with respect to the incorporated diamine: alicyclic, partially aromatic and aromatic diamine (Figure 7-2).

GalX polyamides:

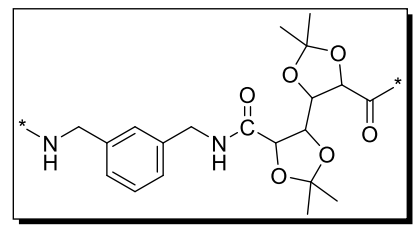

PA(MXD,GalXMe) $M_{n}=21200 \mathrm{~g} / \mathrm{mol}$ $\oplus=2.65$ $T_{g, D S C}=118^{\circ} \mathrm{C}$

Commercial polyamides:

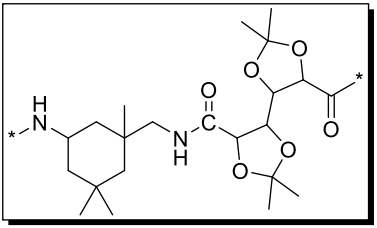

PA(IPDA,GalXMe) $M_{n}=17000 \mathrm{~g} / \mathrm{mol}$ $D=1.70$ $T_{g, D S C}=155^{\circ} \mathrm{C}$

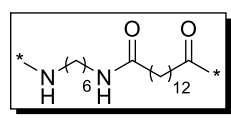

PA(HMDA,DA) $M_{n}=10000 \mathrm{~g} / \mathrm{mol}$ $\oplus=2.20$ $\mathrm{T}_{\mathrm{m}, \mathrm{DSC}}=215^{\circ} \mathrm{C}$

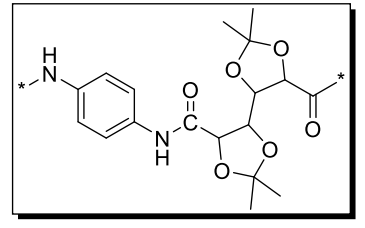

PA(PPDA, GalXMe) $M_{n}=36000 \mathrm{~g} / \mathrm{mol}$ $\Xi=1.67$ $T_{g, D S C}=224^{\circ} \mathrm{C}$

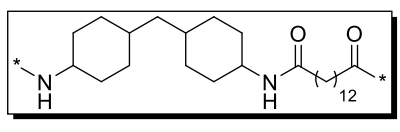

PA(PACM,DA)

$M_{n}=6000 \mathrm{~g} / \mathrm{mol}$ $D=1.65$ $T_{g, D S C}=130^{\circ} \mathrm{C}$

Figure 7-2 Structures and molecular weight data of GalX PAs (PA(MXD,GalXMe), PA(IPDA, GalXMe) and $P A(P P D A, G a l X M e))$ and commercial PAs ((PA(HMDA,DA) and PA(PACM,DA)) which were chosen for the blending experiments. 
Blends were prepared using a twin-screw mini-extruder. Polymers with alicyclic and partially aromatic diamines were easily processable at temperatures above $260{ }^{\circ} \mathrm{C}$ even for elongated time. Polyamides made using aromatic diamine, however, visibly degraded during extrusion at $260-270{ }^{\circ} \mathrm{C}$. In order to evaluate the thermal stability of pure polyamides, the polymers were fed into the extruder chamber at $260-270{ }^{\circ} \mathrm{C}$ and mixed for $1 \mathrm{~min}$. After this time the chamber was evacuated and the degradation was followed by visual evaluation and changes in molecular weight. No discolouration or otherwise manifested degradation was observed regarding PA(IPDA,GalXMe) and PA(MXD,GalXMe) polymers during those experiments (see Figure 7-3 (a) and (b)). The GPC trace of PA(PPDA,GalXMe) shows that the whole distribution shifts towards lower molecular weight values (Figure 7-2 (c.)). This was a decisive factor in the determination of the extruding time, which was eventually limited to 1 minute. The distribution of the molecular weight of the blend is bimodal when there is a big difference in the molecular weight of the components or becomes the average of the two distributions when the difference between the molecular weight of the single components is small. The latter case is applicable for PA(MXD,GalXMe) and PA(IPDA,GalXMe) blends with PA(HMDA,DA) whose difference in molecular weight is between 200-1000 g/mol (Figure 7-3 (a) and (b)). The blending of PA(PPDA,GalXMe) with other polymers suppresses degradation processes, which is seen as an improvement of the appearance of the polymer blend when compared to pure polymer after thermal processing and measured by GPC.

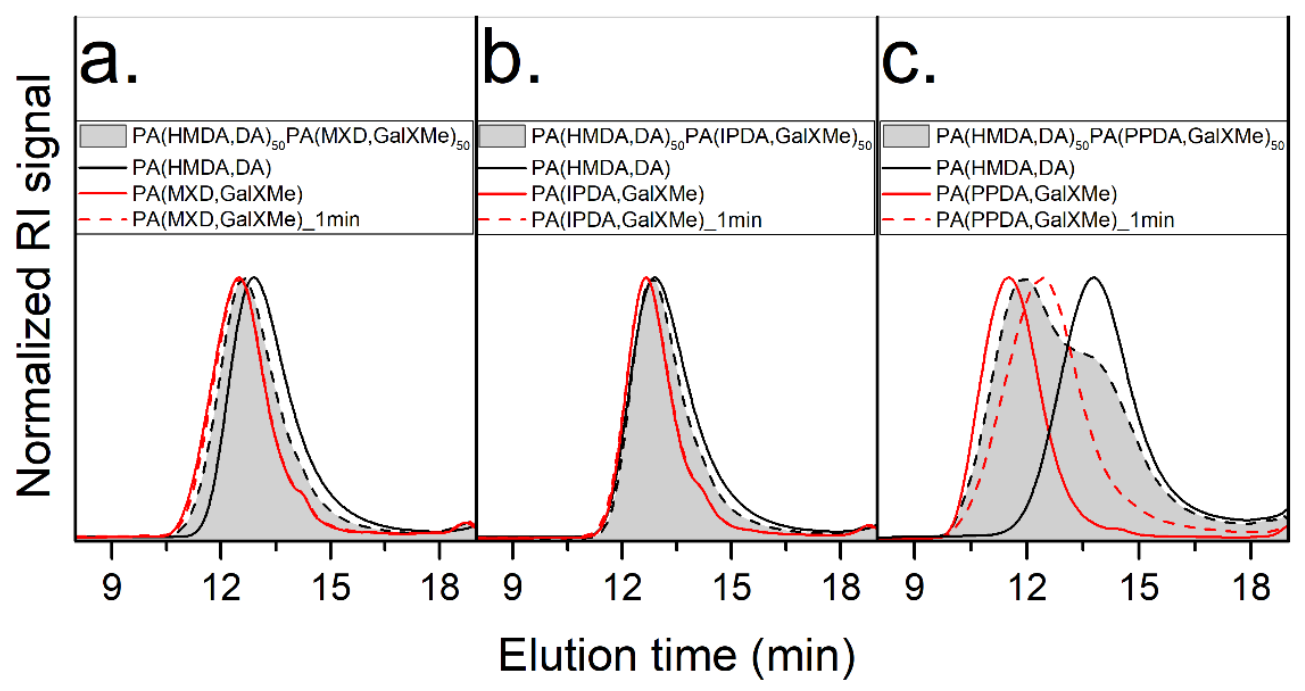

Figure 7-3 Overlay of the GPC traces of the two homopolymers (GalXMe and PA(HMDA,DA)), homopolymer of GalXMe after 1 min of extrusion at $270 / 260^{\circ} \mathrm{C}$ and 50:50 blend of the corresponding homopolymers (a.) PA(MXD,GalXMe), (b.) PA(IPDA,GalXMe) and (c.) PA(PPDA, GalXMe). 


\subsection{Thermal and morphological characterization of blends}

\subsubsection{Thermal properties of PA(HMDA,DA) blends}

Figure 7-4 represent the DSC results recorded for homopolymers and their blends. GalX homopolymers have $T_{\mathrm{g}}$ values of $118{ }^{\circ} \mathrm{C}$ for $\mathrm{PA}(\mathrm{MXD}, \mathrm{GalXMe}), 155{ }^{\circ} \mathrm{C}$ for PA(IPDA,GalXMe) and $224{ }^{\circ} \mathrm{C}$ for PA(PPDA,GalXMe). For PA(MXD,GalXMe) and PA(IPDA,GalXMe) blends with PA(HMDA,DA) the $T_{\mathrm{g}}$ for the GalX component is clearly distinguishable indicating incompatible blends. For a miscible system, only one $T_{g}$ is observed and it is generally between the $T_{\mathrm{g}}$ 's of the pure components. Partial miscibility can be recognized by a shift of the $T_{\mathrm{g}}$ 's compared to the parent polymers. A partially miscible system exhibits two $T_{\mathrm{g}}$ 's that are slightly shifted from that of the neat components. An immiscible system exhibits two $T_{\mathrm{g}}$ 's but exactly that of the neat components. For the blend with PA(PPDA,GalXMe) no glass transition corresponding to the GalX component could be discerned because it overlaps with the melting point of the aliphatic PA(HMDA,DA). Since only the amorphous part in highly crystalline aliphatic polyamides like PA(HMDA,DA) contributes to the glass transition, its $T_{\mathrm{g}}$ could not be detected under the used DSC conditions.

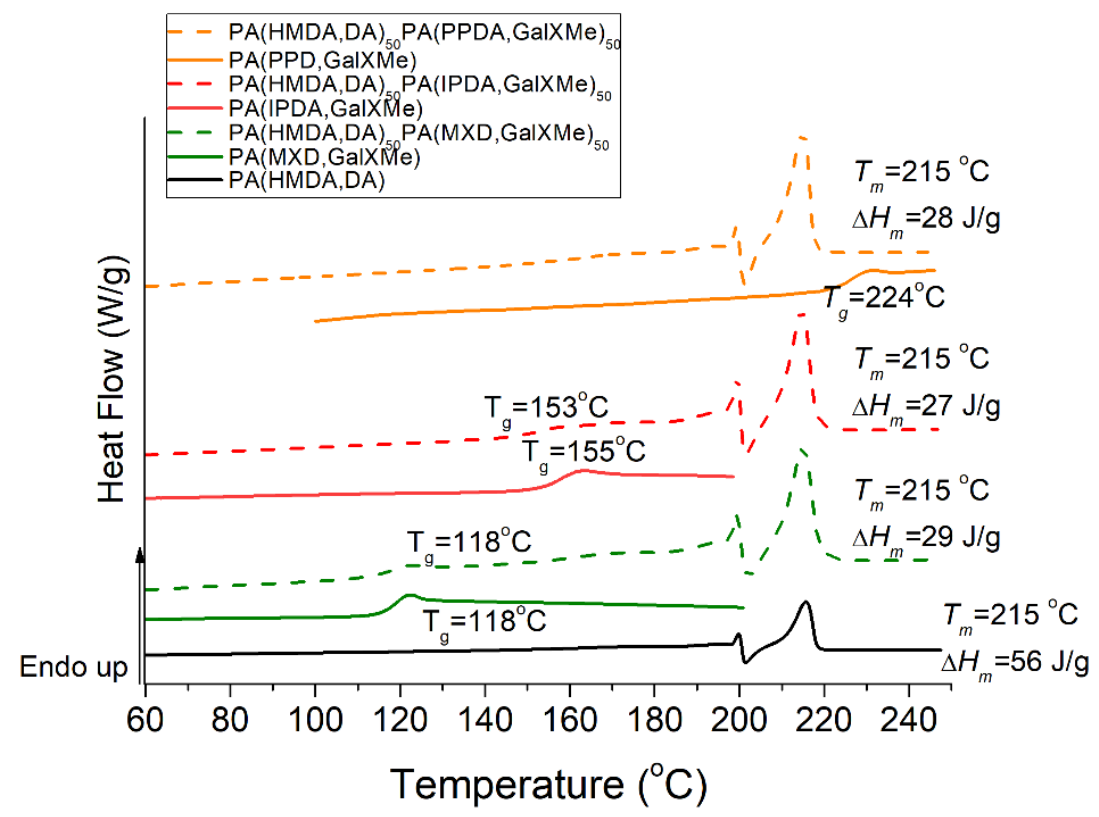

Figure 7-4 DSC curves for homopolyamides and PA(HMDA,DA) blends representing heat flow (W/J) vs. temperature with endothermic exchange up.

\subsubsection{Thermal properties of $P A(P A C M, D A)$ blends.}

Subsequently, blends of PA(PACM,DA) with GalX PAs were investigated (Figure 7-5). Such experiments were limited to using only PA(IPDA,GalXMe) and PA(PPDA,GalXMe) since we would like to focus on the improvement of the thermal and mechanical properties of the 
polyamides and PA(MXD,GalXMe) has a lower $T_{\mathrm{g}}$ than PA(PACM,DA). Initially, DSC curves were recorded for polymer samples after one minute of blending at $270{ }^{\circ} \mathrm{C}$. The analysis revealed that PA(PACM,DDDA) was not compatible with PA(IPDA,GalXMe), as shown by the two separated glass transition regions at $130{ }^{\circ} \mathrm{C}$ and $155{ }^{\circ} \mathrm{C}$, with the first $T_{\mathrm{g}}$ corresponding to amorphous Trogamid CX and the second to PA(IPDA,GalXMe) (Figure 75 (a)). In parallel DSC curves were recorded for PA(PPDA,GalXMe) with PA(PACM,DA) blends, where it was noticed that one $T_{\mathrm{g}}$ was present at values slightly higher than that for the commercial component (from $130{ }^{\circ} \mathrm{C}$ for pure PA(PACM,DA) to $133{ }^{\circ} \mathrm{C}$ for blend after one minute of mixing) (Figure 7-5 (b)), an indication for partial miscibility. This small shift registered for the PA(PPDA,GalXMe) blend stimulated further investigation of the phenomenon. In order to establish whether mixing time influences the miscibility of the components, more samples were prepared for experiments with different mixing times. No influence of time on the quality of mixing was observed for PA(IPDA,GalXMe) polymer blends, which regardless of the time mixing, showed the same transitions corresponding to the individual components. Conversely, PA(PPDA,GalXMe) blended with PA(PACM,DA) showed a correlation of the transition region with the time of mixing: the longer the mixing was applied, the higher the transition region. After 3 minutes of mixing, the glass transition of the prepared blend was increased to $149{ }^{\circ} \mathrm{C}$; however, longer mixing times led to the degradation of the PA(PPDA,GalXMe) polymer blend. As a result of degradation after extended mixing with PA(PPDA,GalXMe) and no influence of time on the miscibility of PA(IPDA,GalXMe) blend, 1 minute of blending time was applied for all subsequent experiments.
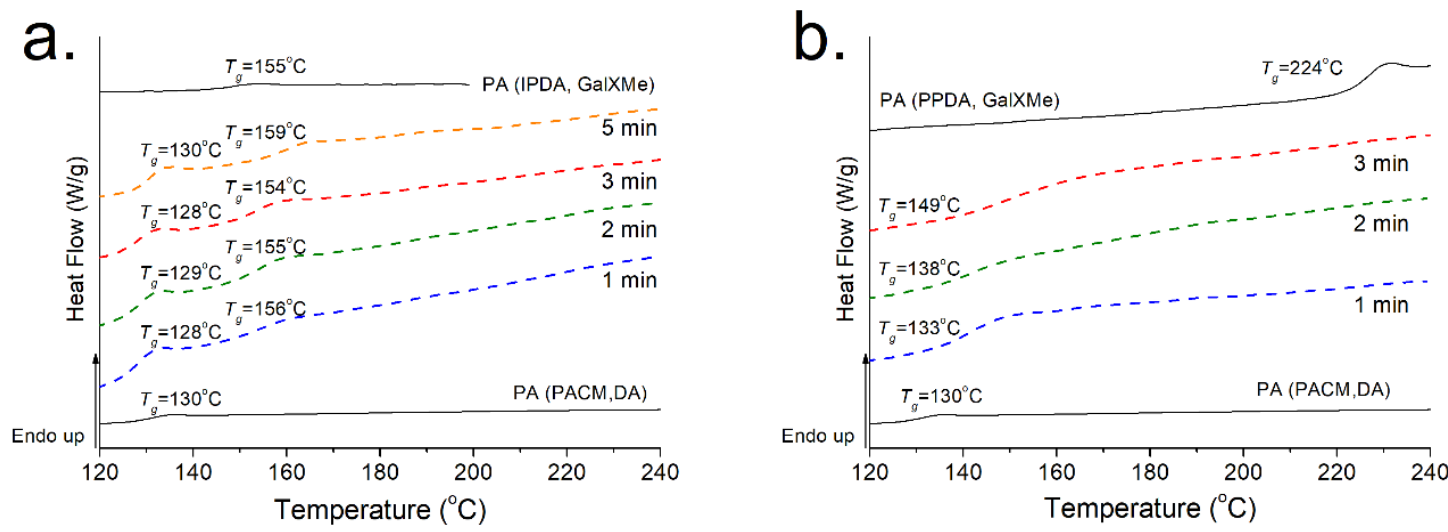

Figure 7-5 DSC curves for blends of homopolyamides with PA(PACM,DA) representing heat flow (W/J) with endothermic exchange up and the variation of the profiles as a function of the proccessing time. (a) for $P A(P A C M, D A)_{50} P A(I P D A, G a l X M e)_{50}$ and (b) PA(PACM,DA ${ }_{50} P A(P P D A, G a l X M e)_{50}$ 


\section{Chapter 7}

\subsubsection{Morphological study of the blends by SEM.}

In principle, in order to achieve a good quality blend, polymers should be miscible with each other. In practice, however, often blending of bio-based polyamides results in an immiscible blend and requires the addition of a compatibilizer to improve polymer dispersion. To investigate miscibility, compatibility, and morphology of blends with PA(HMDA,DA) and PA(PACM,DA), SEM images were collected. Figure 7-6 presents the SEM images of blends with PA(MXD,GalXMe), PA(IPDA,GalXMe) and PA(PPDA,GalXMe). The analysis of microscopic images revealed that blending of PA(MXD,GalXMe) (Figure 7-6(a)) and PA(IPDA,GalXMe) (Figure 7-6(b,d)) results in a two-phase system in PA(HMDA,DA) or PA(PACM,DA). Unlike the aliphatic polyamides, however, PA(PPDA,GalXMe) (Figure 76(c,e) does not show a two-phase system.

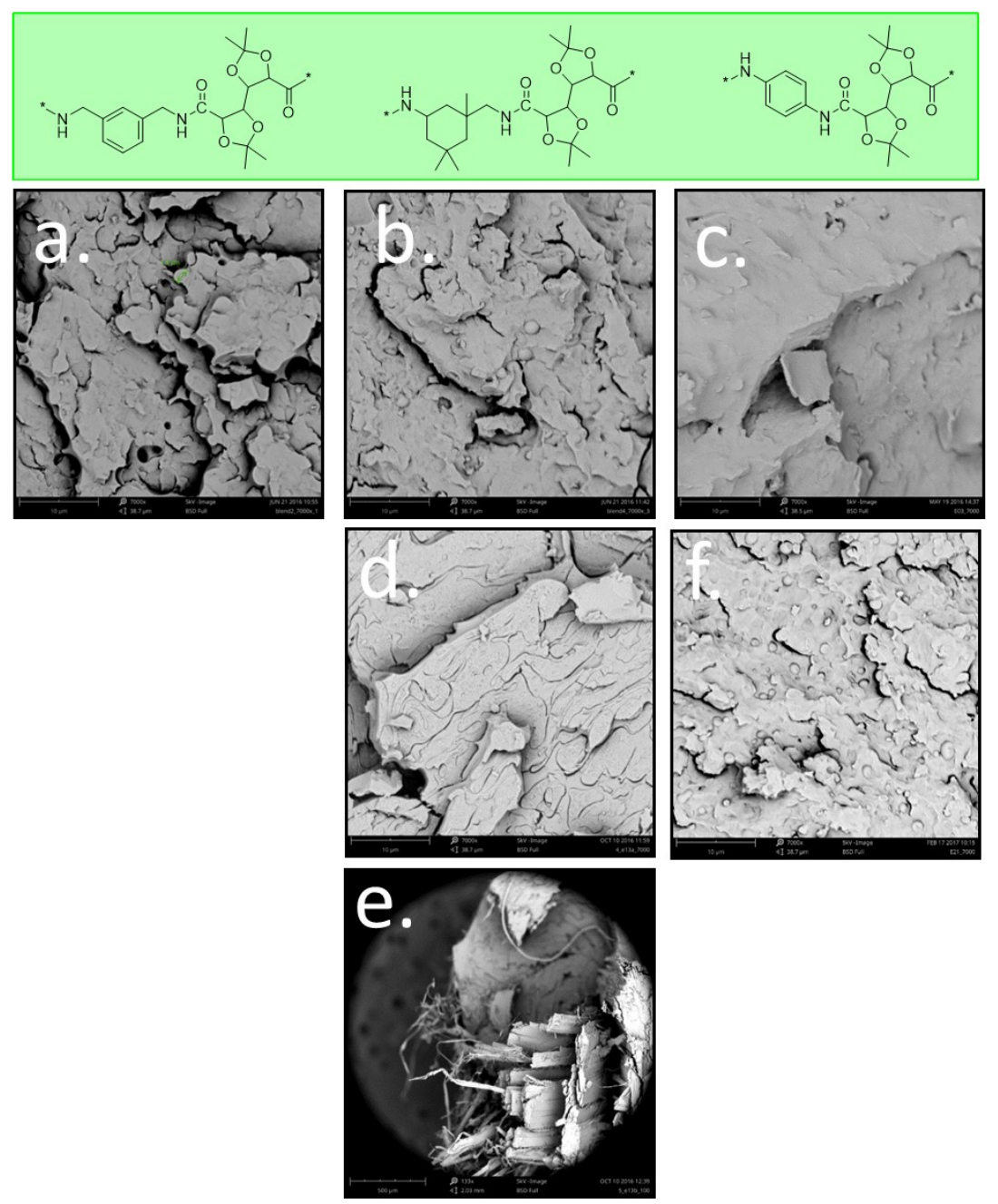

Figure 7-6 SEM images of blends (a) PA(HMDA,DA) ${ }_{50} P A(M X D, G a l X M e)_{50} \quad(x 7000), \quad$ (b) $P A(H M D A, D A)_{50} P A(I P D A, G a l X M e)_{50} \quad$ (x7000), (c)PA(HMDA,DA) $)_{50} P A(P P D A, \text { GalXMe })_{50} \quad(x 7000), \quad(d)$ $P A(P A C M, D A)_{50} P A(I P D A, G a l X M e)_{50}$ (x7000), (e) PA(PACM,DA) $)_{50} P A(I P D A, \text { GalXMe) })_{50}$ (x133), and (f) $P A(P A C M, D A)_{50} P A(P P D A, G a l X M e)_{50}(x 7000)$. 
An interesting phenomenon was observed after analysis of PA(PACM,DA) blends with PA(IPDA,GalXMe). Under microscopic magnification 7000x the blend appears as a twophase system (Figure 7-6 (d)) but under visual inspection the material shows strong orientation, namely the formation of fibers (Figure 7-6(d)). The fibers could not be completely grinded even when ground in liquid nitrogen.

Another investigated aspect of PA(PACM,DA) ${ }_{50} \mathrm{PA}(\mathrm{IPDA}, \mathrm{GalXMe})_{50}$ blends is how the morphology changes as a function of the extrusion time. DSC curves did reveal the indifference of the transition temperatures of those blends towards the extrusion time (Figure 7-7). However, SEM results provide insight into a microscopic effect of time. Figure 7-7 contains 3 SEM images of the blend after 1,2, and 5 minutes blending. The time of extrusion, indeed, reduces the domain sizes. However, even after 5 minutes of blending they are still not compatible.

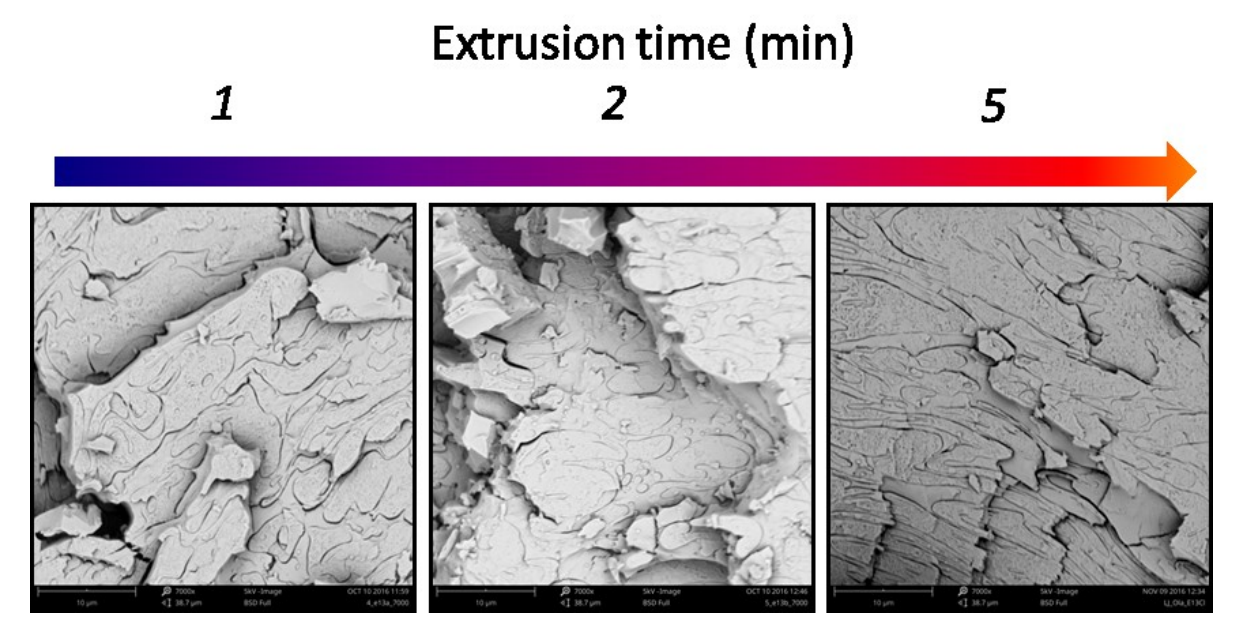

Figure 7-7 SEM images of PA(PACM,DA ${ }_{50} P A(I P D A, \text { GalXMe })_{50}$ blend after 1, 2 and 5 minutes of blending in extruder at $260 / 270^{\circ} \mathrm{C}$.

\subsubsection{Structural transformations induced by thermal processing of PA(PPDA,GalXMe) with $P A(P A C M, D A)$.}

The observed partial compatibility and degradation processes occurring during blending of PA(PPDA,GalXMe) might consist of different phenomena. Thermal degradation of aromatic amine moieties present in the sample might include oxidation of amines or imide formation etc. $^{29,30}$ but in the same time partial miscibility of the PA(PPDA,GalXMe) blends was observed. This suggests that reactions between the two components might have occurred during blending. To elucidate this, 2D ${ }^{1} \mathrm{H}-{ }^{13} \mathrm{C}$ correlation NMR spectroscopy through multiple bonds was used. Spectra were recorded for samples prepared by mixing of the two 


\section{Chapter 7}

polymers (i.e. PA(PPDA,GalXMe) and PA(PACM,DA)) on one hand at room temperature in deuterated solvent and on the other hand after extrusion at $270 / 260{ }^{\circ} \mathrm{C}$ for $3 \mathrm{~min}$. On the ${ }^{1} \mathrm{H}$ NMR spectrum (Figure 7-8 right) and 2D spectrum of the blend prepared at room temperature (RT) peaks from two components (Figure 7-8 left bottom), PA(PPDA,GalXMe) and PA(PACM,DA), are visible. Peaks correlate as follows: the carbonyl group of PA(PPDA, GalXMe) $(\mathrm{C}=\mathrm{O})_{1}$ with protons $\mathrm{H}_{2,3}$, the carbonyl group of $\mathrm{PA}(\mathrm{PACM}, \mathrm{DA})(\mathrm{C}=\mathrm{O})_{\mathrm{a}}$ with protons $\mathrm{H}_{\mathrm{b}}$ and the carbonyl group of the $\mathrm{PA}(\mathrm{PACM}, \mathrm{DDDA})$ end group $(\mathrm{C}=\mathrm{O})_{\mathrm{a}(\text { end group })}$ with protons $\mathrm{H}_{\mathrm{b}}$ (Figure 7-8, bottom). After the extrusion, new peaks are visible (Figure 7-8, top), corresponding to the transamidation reaction between the two polyamides and chain scission. In fact, chain scission might stimulate further degradation processes, which lead to aromatic amine terminated chains and those end groups are the most susceptible for thermal degradation.
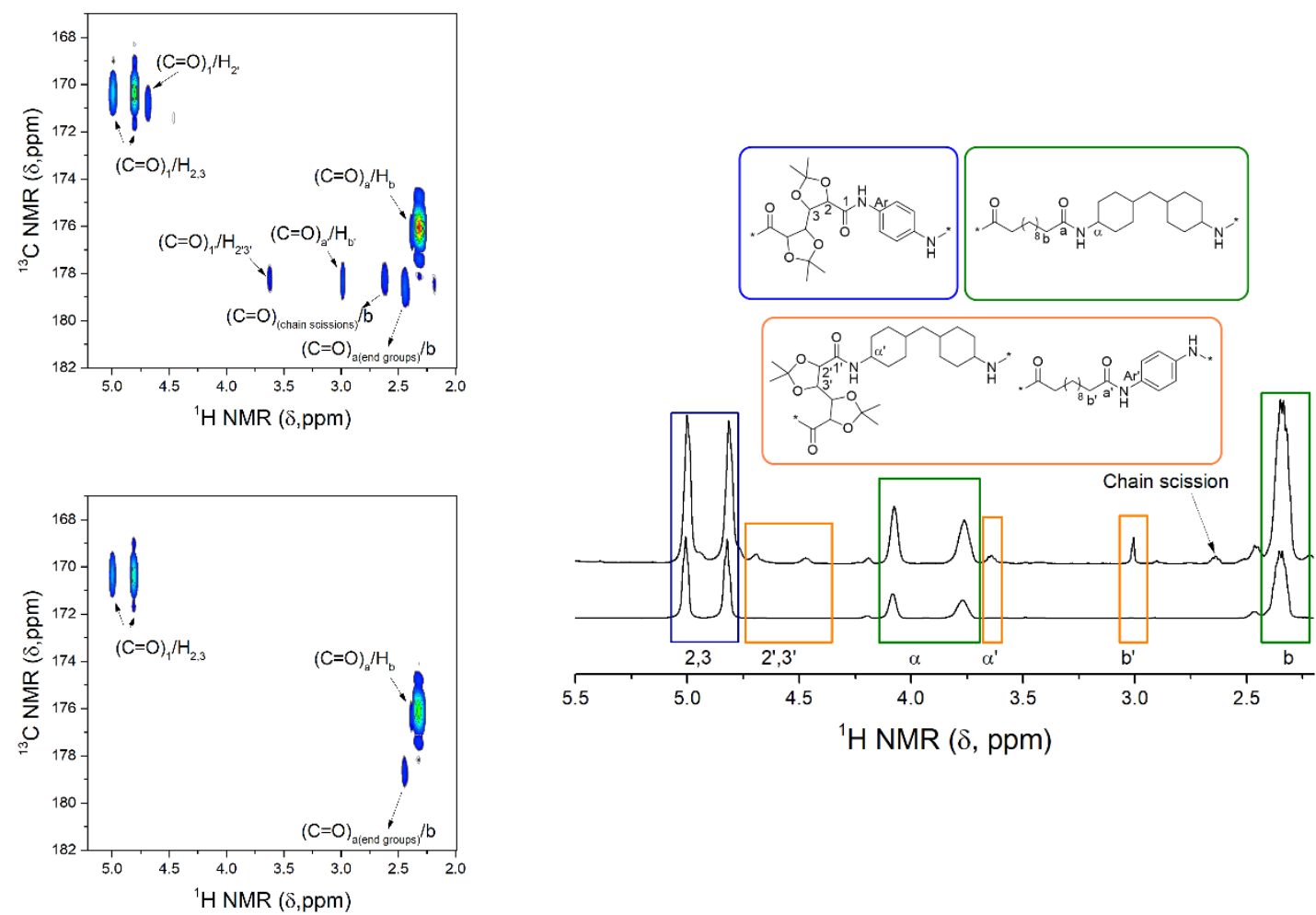

Figure 7-8 The ${ }^{1} \mathrm{H}_{-}{ }^{13} \mathrm{C} \mathrm{HMBC}$ (left) and ${ }^{1} \mathrm{H} N M R$ (right) spectra of PA(PPDA, GalXMe) ${ }_{50} P A(P A C M, D A)_{50}$ blends prepared in the extruder at $260{ }^{\circ} \mathrm{C}$ for $3 \mathrm{~min}$ (top) and in solution at $R T$ (bottom) recorded in a mixture of $\mathrm{CDCl}_{3}$ and $\mathrm{PFP}-\mathrm{d}_{6}$.

The PA(IPDA,GalXMe) spectrum (Figure 7-9) did not show any changes caused by side reactions either before or after extrusion. 

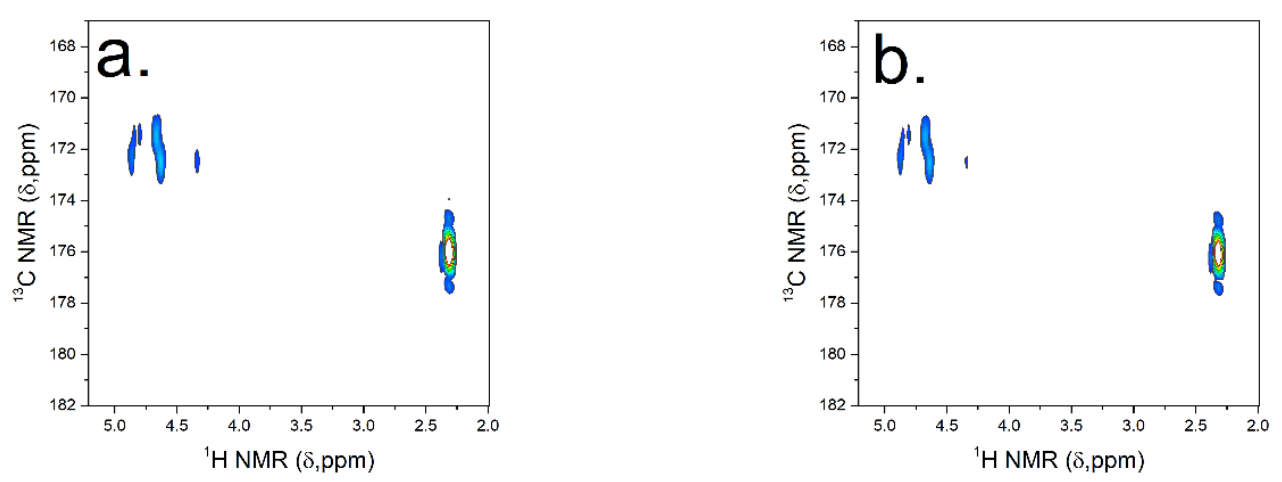

Figure 7-9 The ${ }^{1} \mathrm{H}_{-}{ }^{13} \mathrm{C} \mathrm{HMBC}$ of PA(PACM,DA) ${ }_{50} \mathrm{PA}(\mathrm{IPDA}, \text { GalXMe })_{50}$ blend prepared (a) in the extruder at $260 / 270{ }^{\circ} \mathrm{C}$ for $5 \mathrm{~min}$ and (b) in solution at room temperature recorded in a mixture of $C \mathrm{CCl}_{3}$ and $P F P-d_{6}$.

\subsection{Material properties of blends}

\subsubsection{Mechanical performance of blends.}

Polyamides derived from carbohydrates are known to be highly brittle, which in some cases hampers their mechanical properties. In order to evaluate the influence of such components on the mechanical properties of the blends, tensile tests were conducted. The samples were blended for 1 minute and subsequently injection moulded, resulting in dog-bone-specimens. Table E-1 in Appendix E contains the compiled results of PA(PACM,DA) blends and of PA(HMDA,DA) blends with different GalX polyamides. Due to the high brittleness of the GalX polyamides, it was not possible to obtain tensile bars with more than $50 \mathrm{wt} \%$ of PA(IPDA,GalXMe) and PA(MXD,GalXMe). In all cases, the incorporation of GalX-based polyamides reduced the elastic properties of the material, manifested as a lower elongation at break compared to the non-GalX containing polymer in the blend (see Figure 7-10 (b) and (c)). 

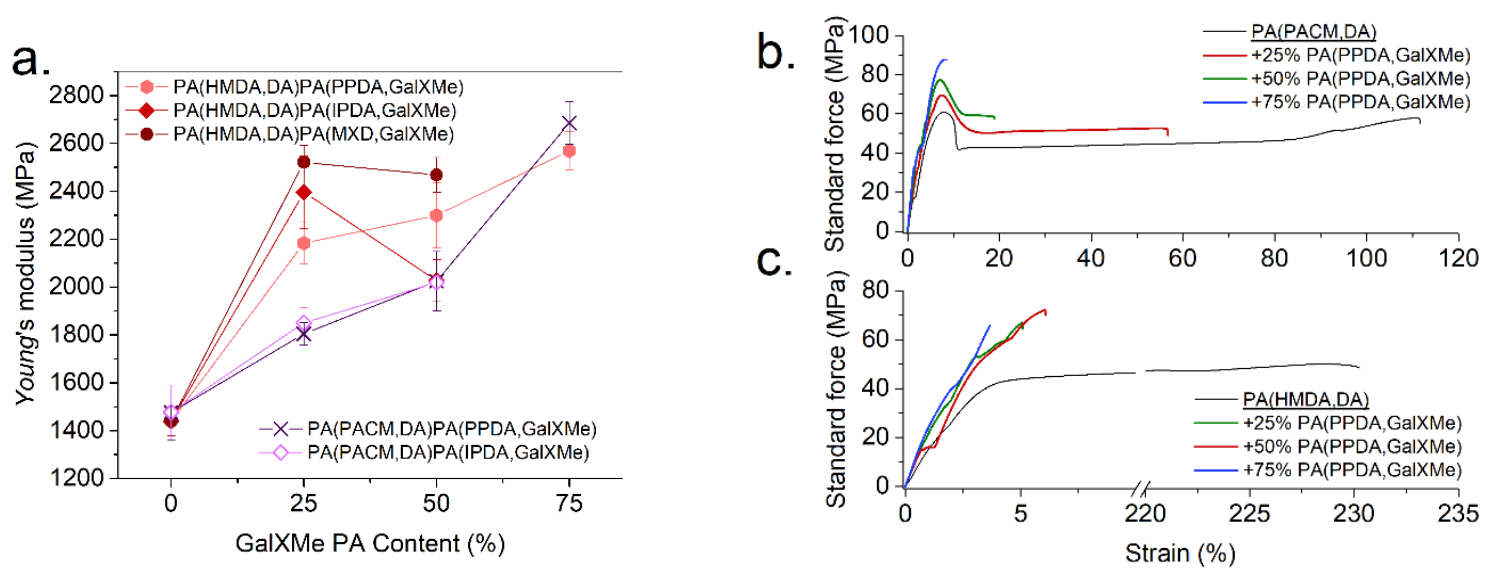

Figure 7-10 Results of the tensile tests for PA(HMDA,DA) and PA(PACM,DA) blended with (a) PA(PPDA,GalXMe), PA(IPDA,GalXMe) and PA(MXD,GalXMe) expressed as E-modulus as a function of the amount of GalXMe polyamide and stress-strain curves for (b) PA(PACM,DA) blended with PA(PPDA, GalXMe) and (c) PA(HMDA,DA) blended with PA(PPDA, GalXMe).

The mixing of GalX polyamides with PA(HMDA,DA) and PA(PACM,DA) significantly improved the $E$ modulus of the prepared blends (Figure 7-10 (a)). In general, with a composition of up to $25 \%$ of carbohydrate-based polyamide, the Young's modulus of the blends increases with respect to the pure commercial polyamide. However, the attempt to blend more than $25 \%$ of such polyamides ended up in the loss of the Young's modulus and a decay in mechanical performance. The PA(PPDA,GalXMe) blends outperform the PA(MXD,GalXMe) and the PA(IPDA,GalXMe) blends and an increased amount of PA(PPDA,GalXMe) in the blend leads to a further increase of the Young's modulus.

As it is demonstrated in Figure 7-10, PA(PPDA,GalXMe) is capable of forming the most promising blend composition, probably correlated with the earlier observed partial miscibility of the blend. Both commercial polyamides PA(HMDA,DA) and PA(PACM,DA), show high elongation at break (Figure 7-10 (b) and (c)) with an E modulus around $1400 \mathrm{MPa}$. Upon the addition of PA(PPDA,GalXMe) polyamide, the $E$ modulus increases and elongation at break decreases. All blends with PA(HMDA,DA) can be classified as hard and brittle materials due to a very low elongation and no yielding. However, the elongation at break of PA(PACM,DA) does not drop that drastically upon the addition of PA(PPDA,GalXMe). Conversely, blends incorporating up to 50\% PA(PPDA,GalXMe) yield upon stretching, with elongation dependent on the amount of added PA(PPDA,GalXMe). Such blends, therefore, can be classified as hard and tough materials. ${ }^{31}$ 


\subsection{Conclusions}

Blends from GalXMe polyamides with semicrystalline PA(HMDA,DA) and amorphous PA(PACM,DA) were successfully prepared by thermal processing in the extruder. The GalXMe polyamides with alicyclic and aliphatic diamines are stable during processing conditions. However, the GalXMe polyamides made with aromatic diamines are susceptible to degradation, particularly under extended processing times.

The blends show no miscibility to partial miscibility with PA(IPDA,GalXMe) and PA(MXD,GalXMe) and partial miscibility with PA(PPDA,GalXMe). PA(PPDA,GalXMe) blends show no phase separation under the SEM microscope, likely as a result of the compatibilizing effect induced by transamidation reactions occurring during the thermal processing in the extruder.

The increased amount of carbohydrate-based component increases the $E$ modulus and decreases the elongation at break of the blends.

\subsection{Materials and methods}

\subsubsection{Materials}

Trogamid CX9704 (Evonik) was purchased from Brenntag (NL). Pentafluorophenol-d 6 99.0\% (PFP- $\mathrm{d}_{6}$ ) and polyamide 6,12 (PA(HMDA,DA)) were purchased from Sigma-Aldrich and used as supplied. 1,1,1,3,3,3-hexafluoro-2-propanol (HFIP) were purchased from Acros Organics. Irgafos 168, Inrganox 1010 were purchased from BASF. $\mathrm{CDCl}_{3}$ was purchased from Cambridge Isotope.

\subsubsection{Methods}

Synthesis of poly(1,3-phenylenedimethanamine-co-2,3:4,5-di-O-isopropylidenegalactaramid) PA(MXD,GalXMe), poly(5-Amino-1,3,3-trimethylcyclohexanemethanamineco-2,3:4,5-di-O-isopropylidene-galactaramid) PA(IPDA,GalXMe) and poly(paraphenylene2,3:4,5-co-di-O-isopropylidene-galactaramid) was conducted according procedures described in Chapter 2.

A typical procedure for blend preparation. PA(MXD,GalXMe) (1.5 g, 50 wt\%), PA(HMDA,DA) (1.5 g, $50 \mathrm{wt} \%$ ) and antioxidants Irganox 1010:Irgafos 168 at a ratio of 1:2 $(0.003 \mathrm{~g}, 0.1 \mathrm{wt} \%)$ were fed into an Xplore MC5 twin-screw mini-extruder under nitrogen atmosphere set with three different temperature zones $270{ }^{\circ} \mathrm{C}$ (top), $260{ }^{\circ} \mathrm{C}$ (middle) and 


\section{Chapter 7}

$260{ }^{\circ} \mathrm{C}$ (bottom) and a screw speed of 100 RPM. Polymers were mixed for 1 minute followed by the evacuation of the chamber to the injection moulding hose at $270{ }^{\circ}$. The mould temperature was set to $50{ }^{\circ} \mathrm{C}$ to obtain dog bone shaped specimens. Injection moulding was performed with an Xplore IM5.5

\subsubsection{Characterization}

Molecular weight of polyamides determined and DSC thermograms were obtained according to the procedure described in Chapter 3.

The $1 D N M R$ spectra were recorded on a Bruker Avance $500 \mathrm{MHz}$ in a mixture of $\mathrm{CDCl}_{3}$ and PFP- $\mathrm{d}_{6}$.

The $2 D-H M B C$ spectra were recorded in a mixture of $\mathrm{CDCl}_{3}$ and PFP- $\mathrm{d}_{6}$. on Bruker $500 \mathrm{MHz}$ using a standard Bruker pulse program hmbcgpl2ndqf with the following parameters: acquisition TD 4096 (f2) x128 (f1), SW = 12.0 ppm (F2), 180 ppm (F1); O1, $12558.27 \mathrm{~Hz}$; O2, $3007.48 \mathrm{~Hz}$; D1 = $1.50 \mathrm{~s}$; CNST6 = 135 CNST7=155; acquisition time, F2 channel, 340.787 ms; F1 channel, 2.827 ms; processing, SI = 1024 (F2, F1), WDW =SINE,

Scanning Electron Microscopy (SEM) analysis of the freeze-fractured samples was done with a PhenomPro apparatus equipped with cold cathode field-emission source and backscattered electron detector (BSD) with optical microscopy magnification up to $135 \mathrm{x}$ and electron magnification of 80-15000x with and acceleration voltage of $5 \mathrm{kV}$. The samples were sputter coated with Au using a Cressington Sputter Coater 108Auto.

Mechanical properties of polymer blends were characterized by performing tensile strength, each in fivefold. Before performing the mechanical tests, the polyamide specimens were dried for $24 \mathrm{~h}$ at $80{ }^{\circ} \mathrm{C}$. Tensile tests were performed with a Zwick/Roell Z020 tensile tester equipped with a $10 \mathrm{kN}$ load cell. The tests were performed on injection moulded samples having a dimension of $75 \mathrm{~mm} \times 4 \mathrm{~mm} \times 2 \mathrm{~mm}$. A grip-to-grip separation of $50 \mathrm{~mm}$ was used. The samples were loaded with a constant crosshead speed of $25 \mathrm{~mm} \cdot \mathrm{min}^{-1}$ 


\section{REFERENCES}

1. S. Spinella, J. Cai, C. Samuel, J. Zhu, S. A. McCallum, Y. Habibi, J. M. Raquez, P. Dubois and R. A. Gross, Biomacromolecules, 2015, 16, 1818-1826.

2. K. Van de Velde and P. Kiekens, Polym. Test., 2002, 21, 433-442.

3. H. Urayama, T. Kanamori, K. Fukushima and Y. Kimura, Polymer, 2003, 44, 5635-5641.

4. C. Lavilla, A. Alla, A. Martínez de Ilarduya, E. Benito, M. G. García-Martín, J. A. Galbis and S. Muñoz-Guerra, J. Polym. Sci., Part A: Polym. Chem., 2012, 50, 3393-3406.

5. C. Lavilla, A. Alla, A. Martínez de Ilarduya, E. Benito, M. G. García-Martín, J. A. Galbis and S. Muñoz-Guerra, J. Polym. Sci., Part A: Polym. Chem., 2012, 50, 1591-1604.

6. E. Gubbels, C. Lavilla, A. M. de Ilarduya, B. A. J. Noordover, C. E. Koning and S. MuñozGuerra, J. Polym. Sci., Part A: Polym. Chem., 2014, 52, 164-177.

7. C. Lavilla, E. Gubbels, A. Martínez de Ilarduya, B. A. J. Noordover, C. E. Koning and S. Muñoz-Guerra, Macromolecules, 2013, 46, 4335-4345.

8. T. Mehtio, L. Nurmi, V. Ramo, H. Mikkonen and A. Harlin, Carbohydr. Res., 2015, 402, 102-110.

9. S. Dhandapani, S. K. Nayak and S. Mohanty, Polymers for Advanced Technologies, 2016, 27, 938-945.

10. E. Enriquez, A. K. Mohanty and M. Misra, Materials \& Design, 2016, 90, 984-990.

11. G. Cicala, A. Latteri, G. Saccullo, G. Recca, L. Sciortino, S. Lebioda and B. Saake, Journal of Polymers and the Environment, 2016, 25, 750-758.

12. M. Reulier and L. Avérous, Eur. Polym. J., 2015, 67, 418-427.

13. Y.-x. Zhou, Z.-g. Huang, X.-q. Diao, Y.-x. Weng and Y.-Z. Wang, Polym. Test., 2015, 42, $17-25$.

14. S. Spinella, C. Samuel, J.-M. Raquez, S. A. McCallum, R. Gross and P. Dubois, ACS Sustainable Chemistry \& Engineering, 2016, 4, 2517-2527.

15. A. Bendaoud, R. Kehrbusch, A. Baranov, B. Duchemin, J. E. Maigret, X. Falourd, M. P. Staiger, B. Cathala, D. Lourdin and E. Leroy, Carbohydr. Polym., 2017, 168, 163-172.

16. C. Lavilla, A. Alla, A. M. de Ilarduya, E. Benito, M. G. Garcia-Martin, J. A. Galbis and S. Munoz-Guerra, Biomacromolecules, 2011, 12, 2642-2652.

17. C. Lavilla, A. Alla, A. Martínez de Ilarduya, E. Benito, M. G. García-Martín, J. A. Galbis and S. Muñoz-Guerra, Biomacromolecules, 2011, 12, 2642-2652.

18. C. Lavilla, A. Alla, A. Martínez de Ilarduya and S. Muñoz-Guerra, Biomacromolecules, 2013, 14, 781-793.

19. C. Lavilla and S. Muñoz-Guerra, Green Chem., 2013, 15, 144-151.

20. C. Lavilla and S. Muñoz-Guerra, Polym. Degrad. Stab., 2012, 97, 1762-1771.

21. S. Munoz-Guerra, High Perform. Polym., 2012, 24, 9-23.

22. S. Muñoz-Guerra, C. Lavilla, C. Japu and A. Martínez de Ilarduya, Green Chem., 2014, 16, 1716-1739.

23. A. A. Wróblewska, K. V. Bernaerts and S. M. A. De Wildeman, Polymer, 2017, 124, $252-$ 262.

24. A. A. Wróblewska, J. Noordijk, N. Das, C. Gerards, S. M. A. Wildeman and K. V. Bernaerts, Macromol. Rapid Commun., 2018, 34, 1800077.

25. C. L. Simões, J. C. Viana and A. M. Cunha, J. Appl. Polym. Sci., 2009, 112, 345-352.

26. J. W. Park and S. S. Im, J. Appl. Polym. Sci., 2002, 86, 647-655.

27. L. Jiang, M. P. Wolcott and J. Zhang, Biomacromolecules, 2006, 7, 199-207.

28. V. Ojijo, S. Sinha Ray and R. Sadiku, ACS Appl Mater Interfaces, 2012, 4, 6690-6701.

29. P. W. Morgan and S. L. Kwolek, Macromolecules, 1974, 8, 104-111.

30. A. P. Gies, S. T. Ellison, S. M. Stow and D. M. Hercules, Anal. Chim. Acta, 2014, 808, 124143.

31. D. W. van Krevelen and K. Nijenhuis, Properties of Polymers:their correlation with chemical structure: their numerical estimation and prediction from additive group contributions., Elsevier, Amsterdam, 2009. 



\section{Chapter 8 VALORIZATION}

Inspired by the possibility to transform waste generated along the food production into useful materials, the author led a reader of this thesis through the straightforward synthesis of homoand copolyamides from natural resources. During this research, attention was given to the properties of polymers as well as to the in-depth analysis of undesired phenomena occurring during the synthesis. Through different parts of this research dissertation, the potential problems regarding the synthesis and subsequent processing of carbohydrate-derived polyamides were discussed and solutions for those problems were proposed. The presented family of compounds, being built from not-yet commercialized building blocks, logically complements the existing set of materials with new combinations of functionalities. In case of building blocks used in this study, belonging to the "GalX" family, these functionalities predominantly encompass high rigidity and good thermal resistance. The incorporation of GalX building blocks generally elevates the glass transition region of copolyamides, while lowering their melting point. Lower melting point facilitates processing which is of considerable significance in the plastics industry. A higher built-in content of cyclic and irregular moieties results in amorphous and transparent polyamides, which adds the esthetic value as it can be used in decorative elements or in manufacturing of materials, for which transparency is a requirement i.e. lamp shades.

The utilization of the renewable sources for the purpose of polymer production has a significant socio-economical aspect. The vast majority of world-wide plastic production is based on the non-renewable fossil industry. ${ }^{1}$ In order to provide sustainable growth of society the long-term availability of resources must constitute the inherent part of the development strategy including alternatives to non-sustainable sources. ${ }^{2,3}$ One efficient strategy is based on the utilization of the biomass to mine chemical compounds, however, not all forms of this approach are entirely justified e.g. plantations require large areas to sustain, which might interfere with agricultural area for food production. Similarly to other fields, the technology to develop this branch of industry started from a not very sustainable but highly efficient route based on the utilization of the world food plantations like the production of bioethanol from sugar-cane. ${ }^{4}$ Naturally, shortly after those plans were executed the monitoring agencies started to send alarming messages regarding the foreseen repercussions of the competition between food resources and the industry. This $1^{\text {st }}$ generation biomass is currently slowly 


\section{Chapter 8}

replaced by $2^{\text {nd }}$ generation biomass, e.g. sugar beet pulp which is a waste product from sugar production. The notable aspect of this transition includes the elimination of the competition between the world food supply and industry, and a clever waste management.

Overcoming humanitarian challenges certainly constitute one of the most important aspects of this research and is part of the long-term crisis prevention. In order to develope usable technology the possible materials from biomass have to be critically evaluated and the optimization of the bio-based materials in such a way that allows achieving the ultimate performance must be performed. Hereby, technology assessment is performed for each section of this research dissertation.

The synthesis of polymers from carbohydrates is often problematic due to the "caramelization" of monomers at higher temperatures. Furthermore, the acetals are compounds, which are generally susceptible to degradation in acidic conditions, thus the adjustment of the synthetic methods is necessary. In Chapter $\mathbf{2}$ and in Chapter $\mathbf{4}$ it is presented in detail which obstacles can be encountered during the preparation of polyamides and alternatives to more troublesome procedures. The presented alternative procedures are industrially relevant and, as a consequence, are foreseen to be predictively transferred to upscaling procedures.

A vast amount of bio-based polyamides contain characteristic amber-like appearance, which by itself is not a major issue except that understanding the origin of the underlying phenomena is of fundamental value. Having better insight into the nature of side reactions further improves the control level over the polymerization and this was analyzed in Chapter 5.

The bio-based polyamides containing a high content of cyclic moieties can be considerably rigid, which could cause the loss of mechanical performance. The produced polymer must be thermally stable not only during the synthesis, but during the processing as well, in order to obtain ready-to-use specimens. The improvement of mechanical properties of materials and their thermal processing is described in Chapter 7 and delivers important information about how to handle bio-based polyamides. 


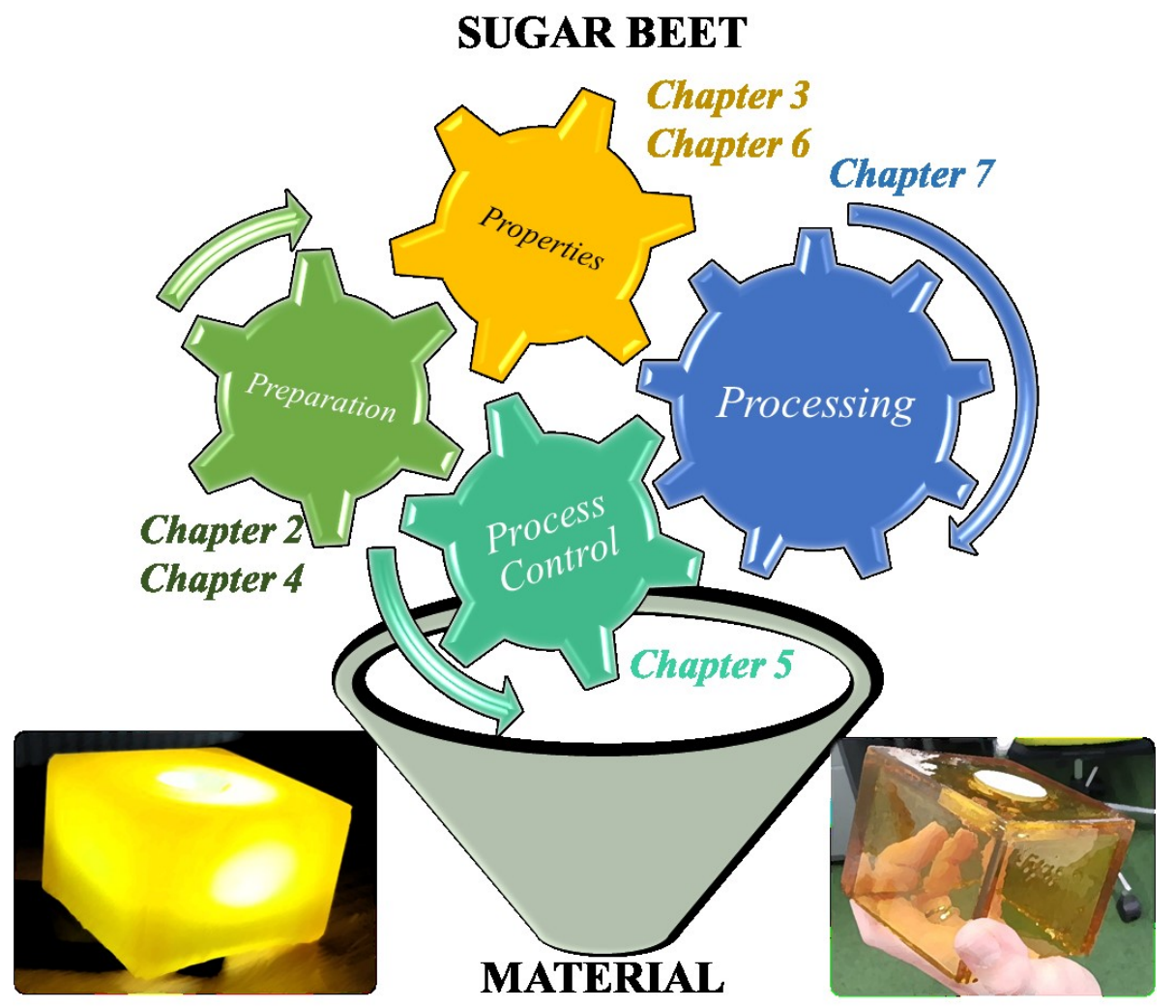

Figure 8-1 The graphical representation of the valorization of the developed technology. GalX polyamides prepared in this project were valorized as lamp shades (left and right bottom pictures).

Lastly, this work can drastically expand the scope of applications of carbohydrate-based materials. Based on the inherent acetal chemistry of GalX building blocks, it might become possible to control which and how many functional groups can be incorporated into a singular monomer structure to be polymerized. This allows for further functionalization of polymers therefrom as well as modification of important parameters i.e. glass transition region or hydrophilicity of polymers. Additionally, the introduction of those moieties into polyamides is changing the balance in different interactions between molecules and chains in the polymeric macro-material. This balance finally helps to determine the general properties of the material. The fundamental understanding of the influence of the protection group onto the reactivity of the flanking carboxyl groups, besides the influence of the cyclic moiety on interactions between polymeric chains, is the topic of Chapter 3 and Chapter 6.

With this valorization addendum this thesis comes to an end. After 4 years of experiments, a simple sugar beet was transformed into a material. Regardless of some left-over flaws, which still need to be solved and a couple of questions, which still need to be answered, the project eventually resulted in the development of technology that is useful and even necessary to 


\section{Chapter 8}

manufacture an expanded set of bio-based polyamide articles, ultimately sourced from local sugar beet resources. 


\section{REFERENCES}

1. M. van den Oever, K. Molenveld, M. van der Zee and H. Bos, Bio-based and biodegradable plastics - Facts and Figures. Focus on food packaging in the Netherlands, Wageningen Food \& Biobased Research, Wageningen, 2017.

2. https://www.european-bioplastics.org/faq-items/how-much-agricultural-area-is-used-forbioplastics/, (accessed 28 March 2018).

3. https://www.european-bioplastics.org/how-much-land-do-we-really-need-to-produce-biobased-plastics/, (accessed 28 March, 2018).

4. K. Srirangan, L. Akawib, M. Moo-Younga and C. P. Choua, Appl. Energy, 2012, 100, 172186. 



\section{APPENDIX A}

Determination of the conversion of functional groups and molar ratio of monomers in

polymer

Example represents calculation for a spectrum collected after 20 minutes of the reaction of the diethyl ester of GalXMe with HMDA (50/50 mol \% feed)

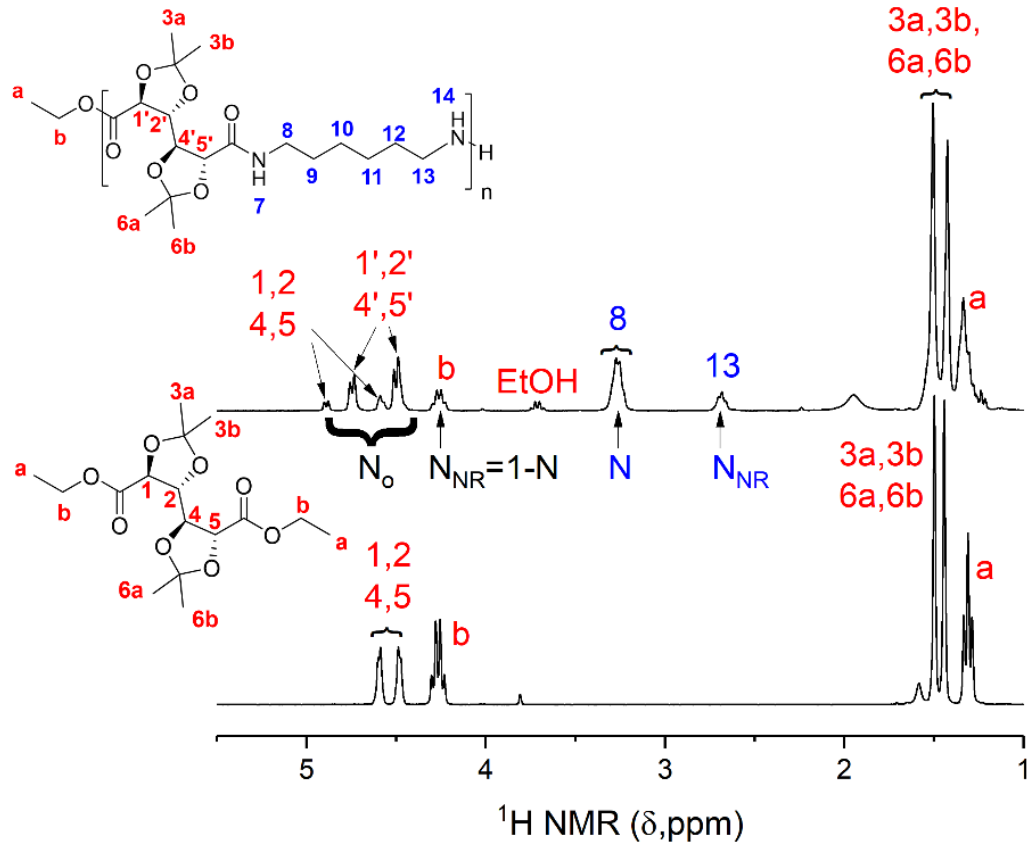

Figure A-1A general procedure for the calculation of the conversion of end groups on an example of partially reacted PA(HMDA, GalXMe) via ${ }^{1} \mathrm{H} N M R\left(300 \mathrm{MHz}, \mathrm{CDCl}_{3}\right.$ solvent).

$$
\operatorname{conv}(\%)=\mathrm{p} \cdot 100 \%=\frac{\mathrm{N}}{N_{o}} \cdot 100 \%=\frac{N_{o}-N_{N R}}{N_{o}} \cdot 100 \%=\left(1-\frac{N_{N R}}{N_{o}}\right) \cdot 100 \%
$$

$\mathrm{N}-$ mol of reacted functional groups, $N_{N R}-$ mol of unreacted functional groups, $N_{o}$ - initial amount of functional groups

$$
\begin{gathered}
\operatorname{conv}(\%) \text { GalX }=\mathrm{p} \cdot 100 \%=\left(1-\frac{N_{N R}}{N_{o}}\right) \cdot 100 \%=\left(1-\frac{2 \frac{N_{b}}{4 H}}{2 \frac{N_{1,2,4,5,1^{\prime}, 2^{\prime}, 4^{\prime} 5^{\prime}}}{4 H}}\right) \cdot 100 \% \\
\operatorname{conv}(\%) \text { diamine }=\mathrm{p} \cdot 100 \%=\frac{\mathrm{N}}{N_{o}} \cdot 100 \%=\frac{\frac{N_{13}}{2 H}}{\frac{N_{8}}{2 H}+\frac{N_{13}}{2 H}} \cdot 100 \%
\end{gathered}
$$


GalX:DIAMINE

$\frac{N_{1^{\prime}, 2^{\prime}, 4^{\prime} 5^{\prime}}}{4 H}: \frac{N_{8}}{4 H}$ 


\section{APPENDIX B}

\section{NMR spectra of monomers and polymers from Chapter 3}
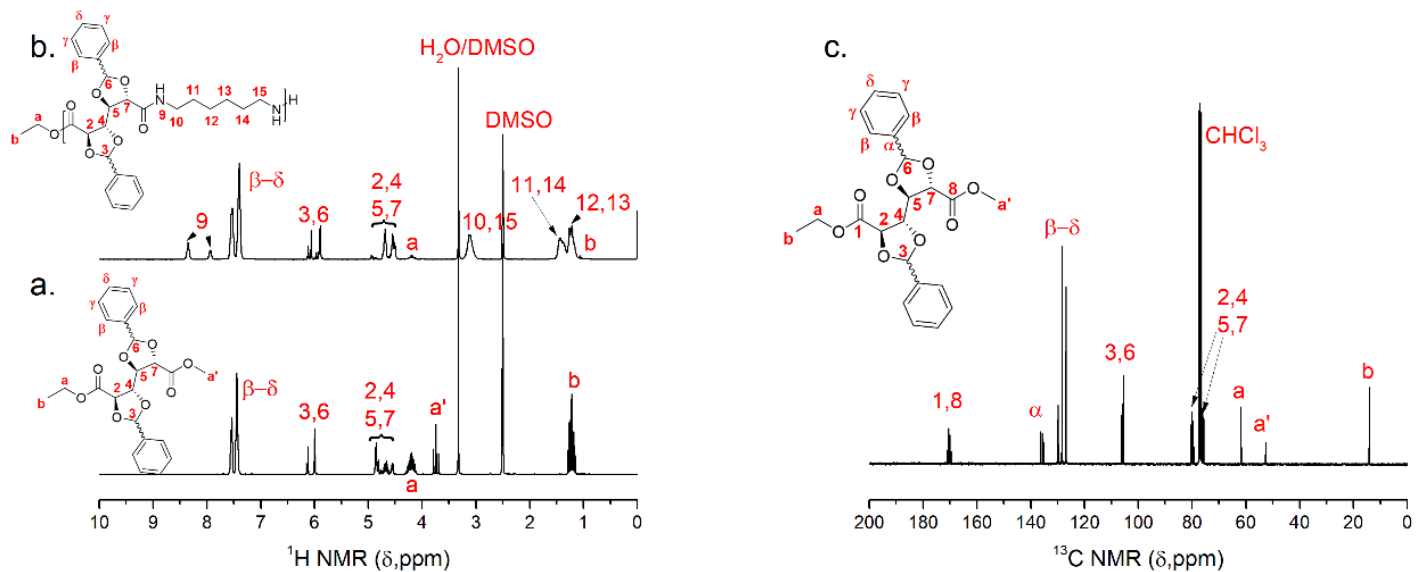

Figure B-1 ${ }^{1} H$ NMR spectra recorded for (a) 2,3:4,5-di-O-benzylidene-galactarate in DMSO- $d_{6}$ and (b) poly(hexamethylene-2,3:4,5-di-O-benzylidene -galactaramide) in DMSO-d ${ }_{6}$ and (c) ${ }^{13} \mathrm{C} N M R$ of 2,3:4,5-di-Obenzylidene -galactarate in DMSO-d6.
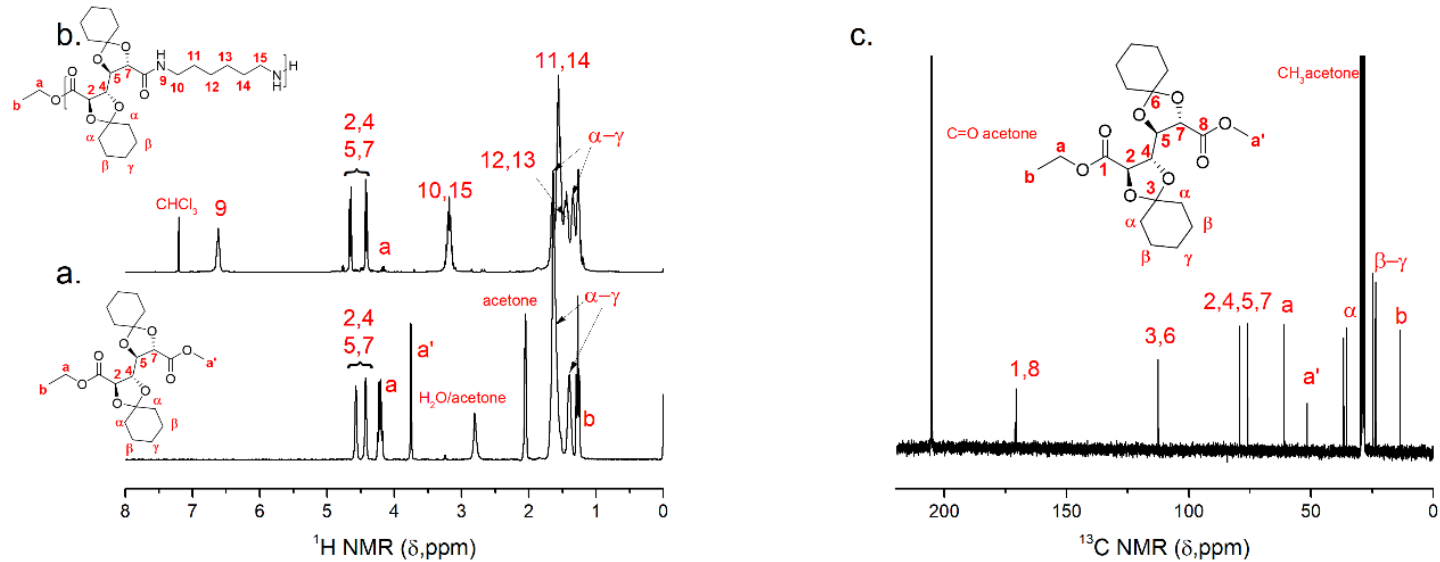

Figure B-2 ${ }^{1} H$ NMR spectra recorded for (a) 2,3:4,5-di-O-cyclohexylidene-galactarate in acetone-d $d_{6}$ and (b) poly(hexamethylene-2,3:4,5-di-O-cyclohexylidene-galactaramide) in $\mathrm{CDCl}_{3}$ and (c) ${ }^{13} \mathrm{C} \mathrm{NMR}$ of 2,3:4,5-di-Ocyclohexylidene-galactarate in acetone- $d_{6}$. 

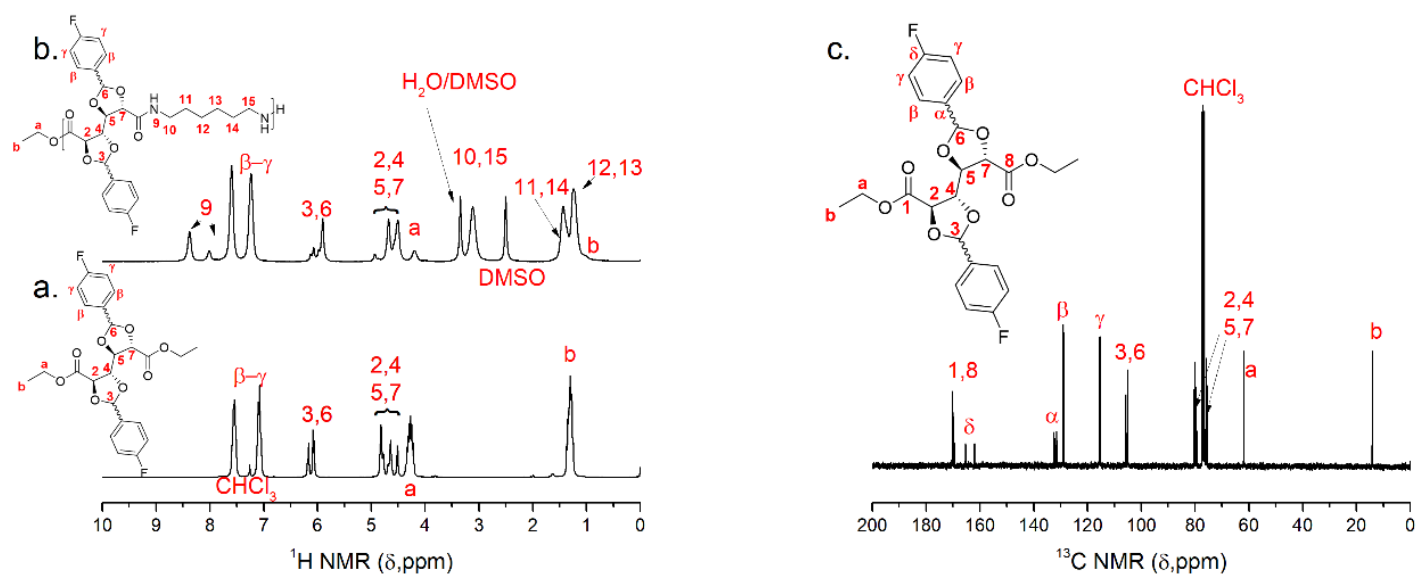

Figure B-3 ${ }^{1} \mathrm{H}$ NMR spectra recorded for (a) 2,3:4,5-di-O-4-fluorobenzylidene-galactarate in $\mathrm{CDCl}_{3}$ and (b) poly(hexamethylene-2,3:4,5-di-O-4-fluorobenzylidene-galactaramide) in $\mathrm{DMSO}-d_{6}$ and (c) ${ }^{13} \mathrm{C} N \mathrm{NM}$ of 2,3:4,5-di-O-4-fluorobenzylidene-galactarate in $\mathrm{CDCl}_{3}$. 


\section{APPENDIX C}

\section{The NMR spectra of copolymers for Chapter 4}
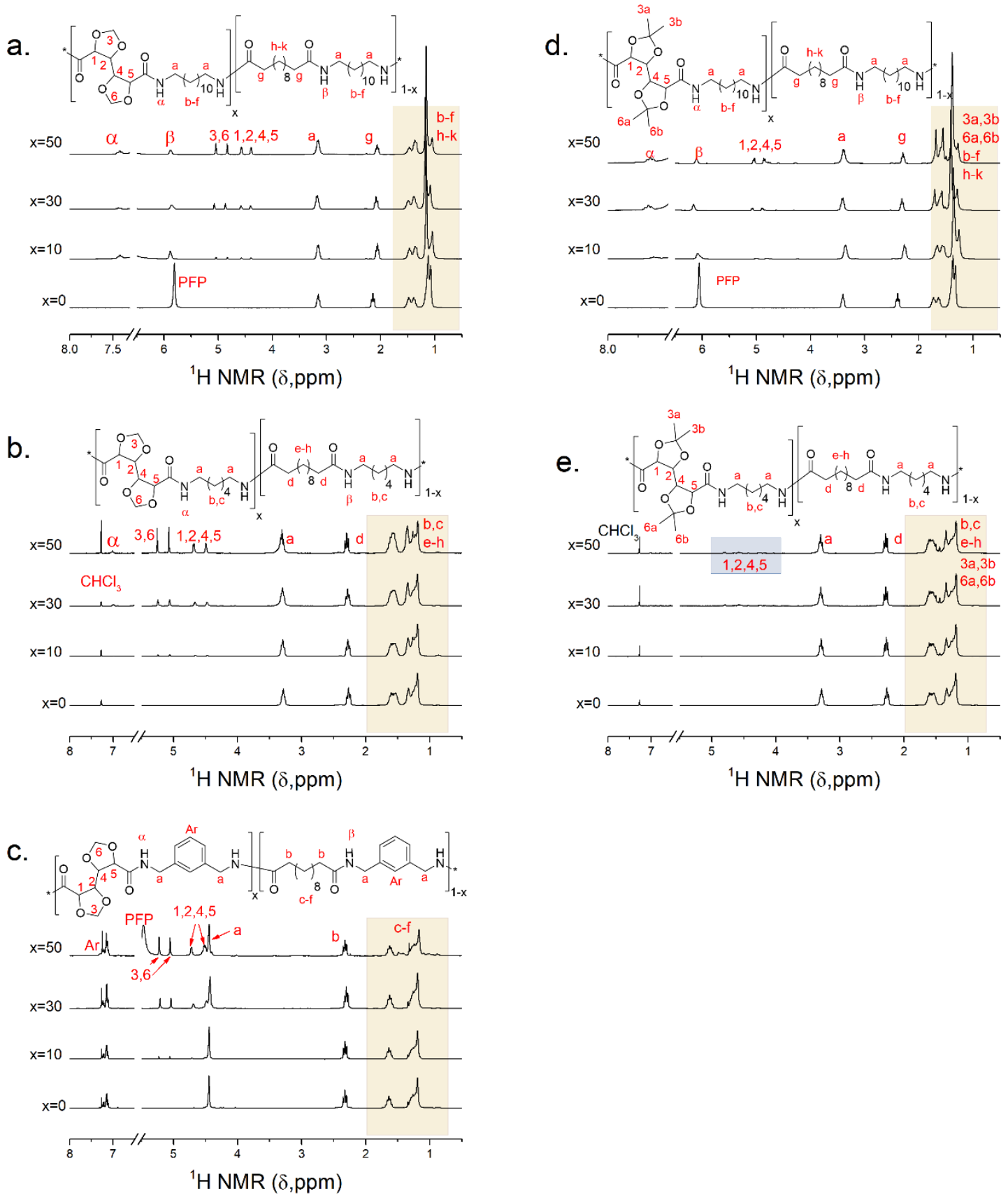

Figure $\quad C-1^{1} H \quad N M R$ of (a) PA(DDDA,GalXH) $)_{x}-c o-P A(D D D A, S A)_{100-x}, \quad$ (b) PA(HMDA,GalXH) $)_{x}-\mathrm{Co}-$ $P A(H M D A, D A)_{100-x}, \quad$ (c) PA(MXD,GalXH) $)_{x}-\mathrm{co}-P A(M X D, D A)_{100-x}$ and (d) PA(DDDA,GalXMe $)_{x}-\mathrm{co}-$ $P A(D D D A, S A)_{100-x}$ and (e) PA(HMDA, GalXMe) $)_{x}$-co-PA(HMDA,DA $)_{100-x}$ series of polyamides recorded in a mixture of pentaflurorophenol-d $d_{1} /$ chloroform- $d_{1} 1 / 4 \mathrm{v} / \mathrm{v} . x=$ mol\% of GalXH salt. Signals below 2 ppm are grouped together (yellow frame). 


\section{APPENDIX D}

The optimized geometries of GalXMe-NH-CH 3 for Chapter 6

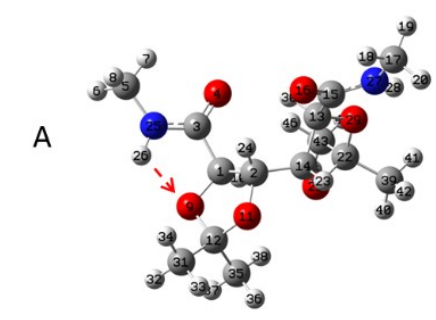

B

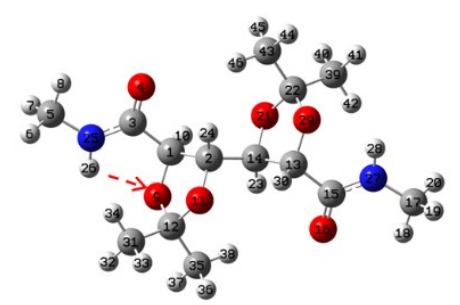

C

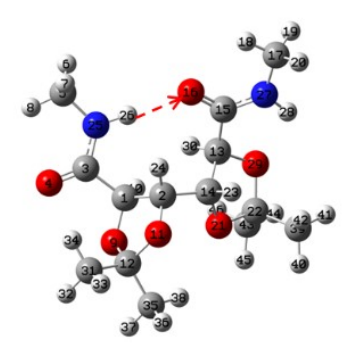

D

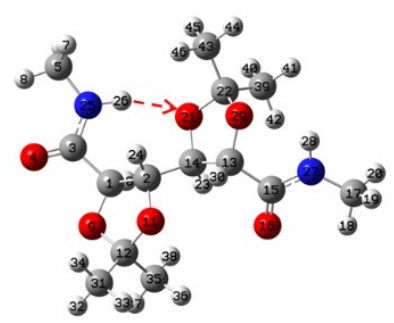

Figure D-1 Optimized structures of conformers A, B, C and D of GalXMe-NHCH and the corresponding intramolecular hydrogen bond formation between hydrogen atom $H_{30}$ and oxygen atom in its vicinity.

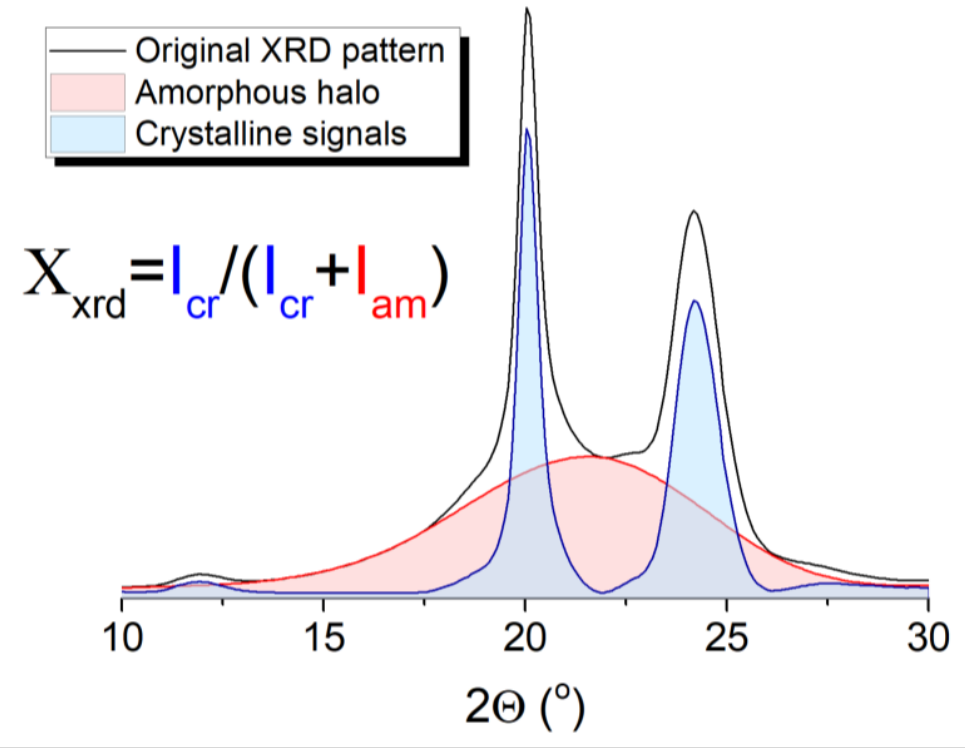

Figure D-2 An example demonstrating the substraction of the baseline from the XRD pattern of crystalline polyamide PA(DDDA,SA). Two parts, crystalline (cr) and amorphous (am), after 


\section{APPENDIXE}

\section{A list of prepared blends from Chapter 7}

Table E-1 The list of the prepared experiments together with E modulus and elongation at break values for Chapter 7. Missing results due to too high rigidity of the material to obtain specimen for test.

\begin{tabular}{|c|c|c|}
\hline Entry* & $E(M P a)$ & $\begin{array}{l}\text { Elongation } \\
\text { at break (\%) }\end{array}$ \\
\hline$P A(H M D A, D A)$ & $1440 \pm 60$ & $237 \pm 30$ \\
\hline$P A(M X D, G a l X M e)$ & - & - \\
\hline$P A(H M D A, D A)_{75} P A(M X D, G a l X M e)_{25}$ & $2520 \pm 70$ & $2 \pm 0.8$ \\
\hline$P A(H M D A, D A)_{50} P A(M X D, G a l X M e)_{50}$ & $2469 \pm 73$ & $3 \pm 0.8$ \\
\hline$P A(H M D A, D A)_{25} P A(M X D, G a l X M e)_{75}$ & - & - \\
\hline PA(IPDA,GalxMe) & - & - \\
\hline$P A(H M D A, D A)_{75} P A(I P D A, G a l X M e)_{25}$ & $2400 \pm 151$ & $3 \pm 0.7$ \\
\hline$P A(H M D A, D A)_{50} P A(I P D A, G a l X M e)_{50}$ & $2030 \pm 88$ & $3 \pm 0.1$ \\
\hline$P A(H M D A, D A)_{25} P A(I P D A, G a l X M e)_{75}$ & - & - \\
\hline$P A(P P D$, GalXMe $)$ & - & - \\
\hline$P A(H M D A, D A)_{75} P A(P P D, G a l X M e)_{25}$ & $2180 \pm 86$ & $4 \pm 0.8$ \\
\hline$P A(H M D A, D A)_{50} P A(P P D, G a l X M e)_{50}$ & $2300 \pm 135$ & $6 \pm 0.7$ \\
\hline$P A(H M D A, D A)_{25} P A(P P D, G a l X M e)_{75}$ & $2570 \pm 80$ & $3 \pm 0.5$ \\
\hline$P A(P A C M, D A)$ & $1480 \pm 114$ & $110 \pm 4.9$ \\
\hline$P A(P A C M, D A)_{75} P A(I P D A, G a l X M e)_{25}$ & $1850 \pm 64$ & $5 \pm 0.8$ \\
\hline$P A(P A C M, D A)_{50} P A(I P D A, G a l X M e)_{50}$ & $2020 \pm 22$ & $4 \pm 0.3$ \\
\hline$P A(P A C M, D A)_{25} P A(I P D A, G a l X M e)_{75}$ & - & - \\
\hline$P A(P A C M, D A)_{75} P A(P P D, G a l X M e)_{25}$ & $1800 \pm 47$ & $56 \pm 2.2$ \\
\hline$P A(P A C M, D A)_{50} P A(P P D, G a l X M e)_{50}$ & $2030 \pm 124$ & $29 \pm 17$ \\
\hline$P A(P A C M, D A)_{25} P A(P P D, G a l X M e)_{75}$ & $2690 \pm 88$ & $6 \pm 2.4$ \\
\hline
\end{tabular}



Rigid, bio-based polyamides from galactaric acid derivatives with elevated glass transition temperatures and their characterization, Wroblewska, Aleksandra A.; Bernaerts, Katrien V.; De Wildeman, Stefaan M. A., Polymer (2017), DOI:10.1016/j.polymer.2017.07.069

In-depth study of the synthesis of polyamides in the melt using biacetal derivatives of galactaric acid. Wróblewska, Aleksandra A.; De Wildeman, Stefaan M.A.; Bernaerts, Katrien V., Polymer Degradation and Stability (2018), DOI: 10.1016/j.polymdegradstab.2018.03.009

Structure-Property Relations in New Cyclic Galactaric Acid Derived Monomers and Polymers Therefrom: Possibilities and Challenges, Wróblewska, Aleksandra A.; Noordijk, Jurrie; Das, Nick; Gerards, Chris; De Wildeman; Stefaan M.A.; Bernaerts, Katrien V. Macromolecular Rapid Communications (2018), DOI: 10.1002/marc.201800077

A solvent-free method for the copolymerization of labile sugar-derived building blocks into polyamides. Wróblewska, Aleksandra A.; De Wildeman, Stefaan M.A.; Stevens, Sander; Garsten, Wessel; Bernaerts, Katrien V. Sustainable Chemistry \& Engineering (2018), DOI: 10.1021/acssuschemeng.8b03587

Carbohydrate-derived polyamides and their processability - from sugar beet to material, Wróblewska, Aleksandra A.; Leone, Nils; De Wildeman, Stefaan M.A.; Bernaerts, Katrien V. To be submitted

Cyclic dioxolane moieties in polyamides - the effect of the additional oxygen and the bulky cyclic structure on the global interactions between polymeric chains, Wróblewska, Aleksandra A.; Harings, Jules; De Wildeman, Stefaan M.A.; Bernaerts, Katrien V. To be submitted

Polyamides based on a partially bio-based spirodiamine, Wróblewska, Aleksandra A.; Lingier, Sophie; Noordijk, Jurrie; Du Prez, Filip E.; De Wildeman, Stefaan M.A.; Bernaerts, Katrien V. European Polymer Journal (2017) DOI: 10.1016/j.eurpolymj.2017.08.056*

Towards sugar-derived polyamides as environmentally friendly materials, Wróblewska, Aleksandra; Zych, Arkadiusz; Thiyagarajan, Shanmugam; Dudenko, Dmytro; van Es, Daan; Hansen, Michael Ryan; Koning, Cor; Duchateau, Rob; Jasinska-Walc, Lidia, Polymer Chemistry (2015) DOI: 10.1039/c5py00521c*

*Publication does not constitute any part of this thesis 



\section{ACKNOWLEDGEMENTS}

I joined this research project in September 2014, which from today's perspective seems much shorter that it actually was. Largely due to the fantastic atmosphere and amazing team, I had the pleasure to work with. I would like to thank my colleagues for all the fruitful discussions and shared relaxing time.

Foremost, I would like to acknowledge Dr. Katrien Bernaerts and Prof. Stefaan De Wildeman. Katrien, thank you for all shared knowledge, all your support and all the scientific challenges, which you placed in front of me, that stimulated my professional development. Stefaan, thank you for your support, motivation and inspiration. After discussions with you the term biobased has become more substantial and meaningful.

I would like to express my gratitude to the members of my reading committee Prof. Lorenzo Moroni, Prof. Francesco Picchioni, Dr. Hanne Diliën and Dr. Sepas Satayesh, for accepting to be part of the committee and taking time to review my thesis.

I would like to acknowledge the input of all those fantastic researchers and extremely helpful technical staff (Jurrie Noordijk, Nick Das and Nils Leoné), with whom I had the pleasure to work through all those years. Especially, Prof. Sanjay Rastogi, Dr. Jules Harings, Gijs de Kort, who advised me on certain parts of the thesis.

I would like to acknowledge all members of my consortium for the interesting discussions and stimulating questions during our meetings and the funding agency for the financial support. I was lucky to have the opportunity to take part in this initiative.

Special thanks to my unofficial reading committee Marie, Thomas and Jurrie.

Each project has its brain and its muscles and we cannot forget about the muscles. Special thanks to Sander, Jeroen, Wessel, Chris, Rocio and George for their contribution. I hope I gave you as much as you gave me.

Thijsje (and PP), thank you for being with me in this enjoyable and sometimes challenging time of my life... Well, Our life... I would also like to thank my Polish and Dutch family. Ohhh, and Ricky of course. Oh, and Dominika for the cover. Oops, I forgot about Ksenia, Elles, Mieke, Marta and Patrycja. And so on... 
Na zakończenie zostawiłam najważniejsza dedykację. W zasadzie stanowi ona dedykację, której rama czasowa wychodzi daleko poza ostatnie cztery lata mojego życia, spędzone na przeprowadzaniu badan naukowych. Mamo i Tato, w tym wzruszającym momencie, w którym to piszę, tza kręci mi się $w$ oku. Tyle ekscytujacych momentów mojego życia, łacznie z ukończeniem doktoratu, nie byłoby możliwe bez Waszego wsparcia, jak również bez nauk, które od Was otrzymałam. Jesteście najkochańszymi rodzicami i najmadrzejszymi nauczycielami, jakich mogłam sobie wymarzyć. Dziękuję.

\section{Hartstikke Bedankt}




\section{CURRICULUM VITAE}

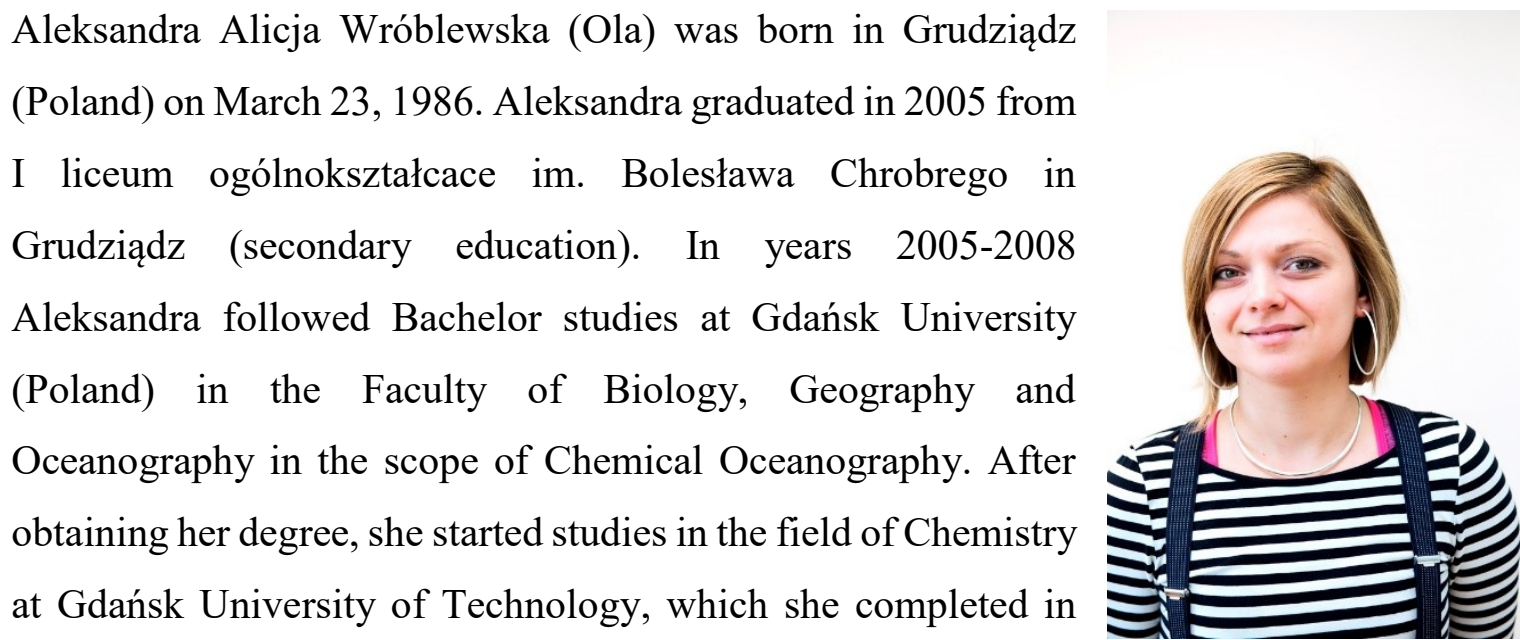

2012 with Bachelor Degree of Engineer, followed by Master Study in the field of Chemical Technology with specialization Organic Technology. During this time, she began to work on her Master thesis under the supervision of Dr. Lidia Jasińska -Walc on a synthesis of polyamides from isohexide derivatives - cyclic molecules derived from starch. During her internship at Sabic R\&D in Geleen (the Netherlands) in years 2013-2014 she was developing compatibilizers for polymer blends via postreactor modification of polyolefins. Directly after the internship she started her $\mathrm{PhD}$ under the supervision of Prof. Stefaan De Wildeman and Dr. Katrien Bernaerts, focusing on the development of polyamides from biacetalized derivatives of galactaric acid. The scope of her study included the synthesis of polymers and characterization of their properties. During this time she attended several courses expanding her understanding of X-ray diffraction in Delft, the Netherlands (2017) organized by the Dutch Crystallographic Society and two courses about the electronic structure methods in Santiago, Spain (2017) organized by Gaussian and in Gent, Belgium (2015) organized by Gent University. Starting from October 2018 Aleksandra will continue an academic carrier at Gent University (Belgium) in the group of Prof. Filip Du Prez. 